\title{
INVESTIGATING VOCABULARY IN ACADEMIC SPOKEN ENGLISH: CORPORA, TEACHERS, AND LEARNERS
}

BY

THI NGOC YEN DANG

\author{
A thesis \\ submitted to the Victoria University of Wellington \\ in fulfilment of the requirements for the degree of \\ Doctor of Philosophy \\ in Applied Linguistics
}

Victoria University of Wellington 



\begin{abstract}
Understanding academic spoken English is challenging for second language (L2) learners at English-medium universities. A lack of vocabulary is a major reason for this difficulty. To help these learners overcome this challenge, it is important to examine the nature of vocabulary in academic spoken English.
\end{abstract}

This thesis presents three linked studies which were conducted to address this need. Study 1 examined the lexical coverage in nine spoken and nine written corpora of four well-known general high-frequency word lists: West's (1953) General Service List (GSL), Nation's (2006) BNC2000, Nation's (2012) BNC/COCA2000, and Brezina and Gablasova's (2015) New-GSL.

Study 2 further compared the BNC/COCA2000 and the New-GSL, which had the highest coverage in Study 1. It involved 25 English first language (L1) teachers, 26 Vietnamese L1 teachers, 27 various L1 teachers, and 275 Vietnamese English as a Foreign Language learners. The teachers completed 10 surveys in which they rated the usefulness of 973 non-overlapping items between the BNC/COCA2000 and the NewGSL for their learners in a five-point Likert scale. The learners took the Vocabulary Levels Test (Nation, 1983, 1990; Schmitt, Schmitt, \& Clapham, 2001), and 15 Yes/No tests which measured their knowledge of the 973 words.

Study 3 involved compiling two academic spoken corpora, one academic written corpus, and one non-academic spoken corpus. Each contains approximately 13-million running words. The academic spoken corpora contained four equally-sized sub-corpora. From the first academic spoken corpus, 1,741 word families were selected for the Academic Spoken Word List (ASWL). The coverage of the ASWL and the BNC/COCA2000 in the four corpora and the potential coverage of the ASWL for learners of different vocabulary levels were determined.

Six main findings were drawn from these studies. First, in the first academic spoken corpus, the ASWL and its levels had slightly higher coverage in certain disciplinary sub-corpora than in the others. Yet, the list provided around 90\% coverage of each subcorpus. It helps learners to achieve $92 \%-96 \%$ coverage of academic speech depending on their levels. Second, the BNC/COCA2000 is the most suitable general highfrequency word list for L2 learners from the perspectives of corpus linguistics, teachers, and learners. It provided higher coverage than the GSL and the BNC2000, and had 
more words known by learners and perceived as being useful by teachers than the NewGSL. Third, general high-frequency words, especially the most frequent 1,000 words, provided much higher coverage in spoken corpora than written corpora in both academic and non-academic discourse. Fourth, despite the importance of general highfrequency words, a reasonable proportion of the learners had insufficient knowledge of these words, which highlights the importance of a word list which is adaptable to learners' proficiency like the ASWL. Fifth, lexical coverage had significant but small correlations with teacher perception of word usefulness and learner vocabulary knowledge. Sixth, the Vietnamese L1 teachers had the highest correlation between the teacher ratings of word usefulness and the learner vocabulary knowledge. Next came the various L1 teachers, and then the English L1 teachers.

This thesis also provides theoretical, pedagogical, and methodological implications of these findings so that L2 learners can gain better support in their vocabulary development and achieve better comprehension of academic spoken English. 


\section{Acknowledgements}

I would like to express my sincerest gratitude to my two supervisors, Dr. Averil Coxhead and Professor Stuart Webb, for their great patience, invaluable guidance, and generous support during my $\mathrm{PhD}$. It is my great honour to have them as mentors. Working with them helps me become a stronger researcher and grow as a person. My special thanks to Emeritus Professor Paul Nation for helping me to achieve better insight into the nature of word list studies and the RANGE program. It is always a great pleasure to talk and learn from him about vocabulary research.

I would like to express my heartfelt thanks to all the teacher and learner participants for their enthusiasm and insights during my project. Also, my great thanks to the following publishers and researchers for their generosity in letting me use their materials to create my corpora: Cambridge University Press, Pearson, Dr. Lynn Grant (Auckland University of Technology), Assistant Professor Michael Rodgers (Carleton University), the lecturers at Victoria University of Wellington, the researchers in the British Academic Spoken English corpus project, the British Academic Written English corpus project, the International Corpus of English project, the Massachusetts Institute of Technology Open courseware project, the Open American National corpus project, the Santa Barbara Corpus of Spoken American-English project, the Stanford Engineering Open courseware project, the University of California, Berkeley Open courseware project, and the Yale University Open courseware project. Without this support, it would be impossible for me to complete this thesis.

I am especially indebted to Victoria University of Wellington for supporting my research financially in the form of Victoria Doctoral Scholarship, Postgraduate Research Excellence Award, Faculty Research Grant, and Victoria Doctoral Submission Scholarship.

Parts of Section 2.8, Chapter 3, and Section 6.2 have been adapted for a joint authored article (with Professor Stuart Webb as the second author) under the title 'Evaluating lists of high frequency words' that appeared in the ITL International Journal of Applied Linguistics, 167(2) (2016), 132-158. I am very grateful to the Editor and the anonymous Reviewers of this journal for their useful feedback on the article.

My sincere thanks go to Professor Laurence Anthony (Waseda University) and Dr. Anne O'Keeffe (University of Limerick) for suggesting some sources of academic 
spoken materials, Professor Hilary Nesi (Coventry University) for the useful information about the British Academic Spoken English Corpus and her Spoken Academic Word List, Dr. Lisa Wood (School of Mathematics and Statistics) and Dr. Vlav Brezina (University of Lancaster) for helping me understand more about the nature of some statistical formulas, and Dr. Deborah Laurs, Kirsten Reid, and Emma Rowbotham (Student Learning Support Service) for their useful advice on my writing and oral presentation skills.

My warmest thanks to all the people in Vietnam and New Zealand who have helped and supported me so far, from family members, friends, officemates, Vocab Group members, Thesis Group members, and staff at the School of Linguistics and Applied Language Studies, Victoria University of Wellington, and colleagues at the University of Languages and International Studies, Vietnam National University, Hanoi.

My deepest gratitude to Mum and Professor Stuart Webb, the two people who have the greatest influence on my direction of life and ways of thinking. I dedicate this work to them for their continual support, encouragement, trust, and guidance during these times and always. 


\section{Table of Contents}

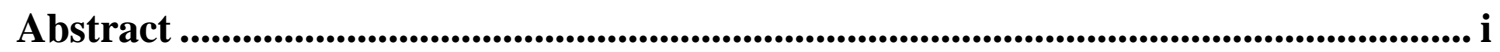

Acknowledgements .................................................................................................................................... iii

Table of Contents....................................................................................................

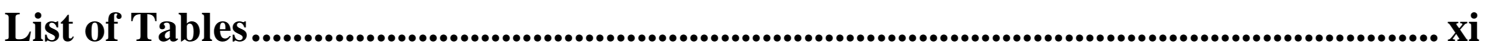

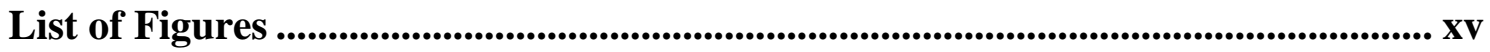

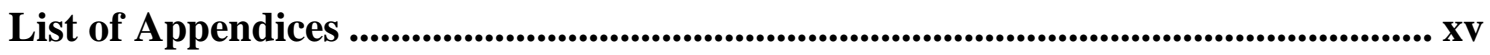

List of Abbreviations ..................................................................................................

Chapter 1 - Introduction .................................................................................. 1

1.1. Why investigate vocabulary in academic spoken English? ................................. 1

1.2. Why investigate general high-frequency vocabulary when examining academic

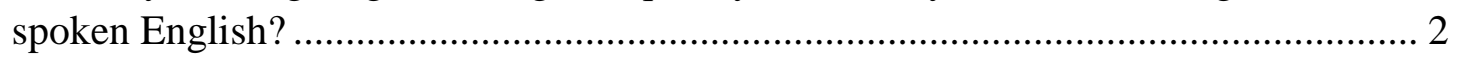

1.3. Why investigate corpora, teachers, and learners? ............................................. 3

1.4. Aims and scope of the present research ........................................................... 4

1.5. Significance of the present research ................................................................ 5

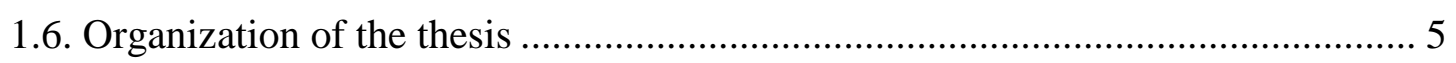

Chapter 2 - Literature Review....................................................................................... 7

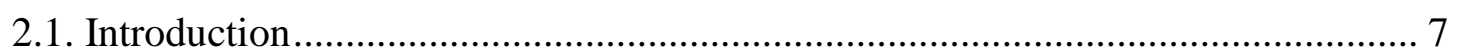

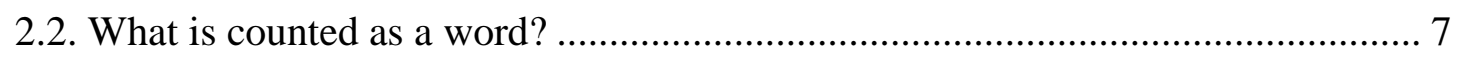

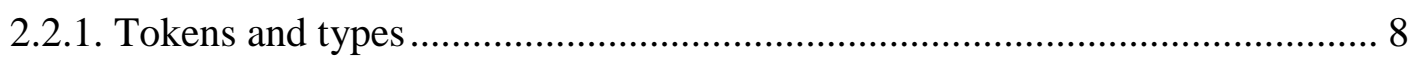

2.2.2. Lemmas and flemmas............................................................................ 8

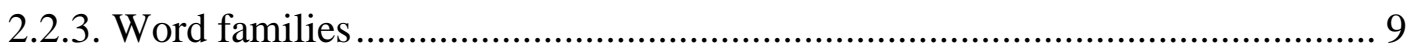

2.2.4. What is the most suitable unit of counting in a word list? ........................... 11

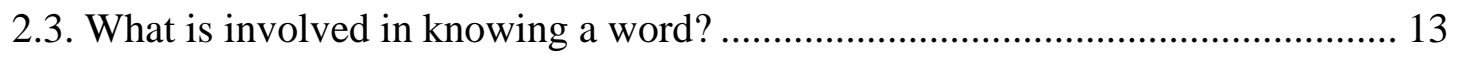

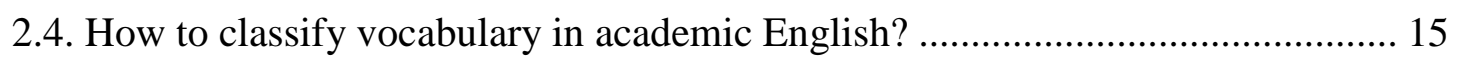

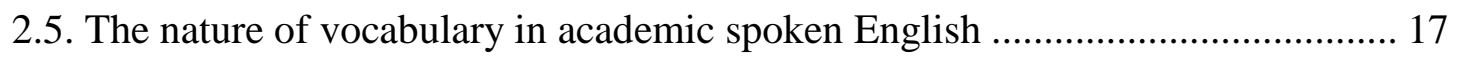

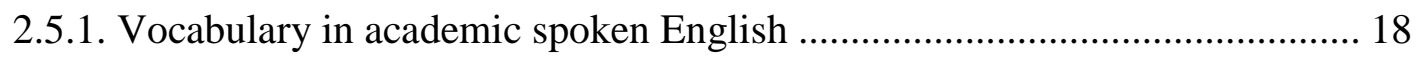

2.5.2. Vocabulary in academic written English ................................................ 23

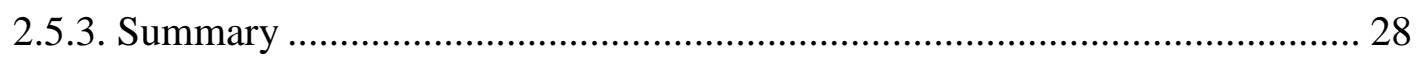

2.6. Teacher perception in word list validation ..................................................... 28

2.6.1. Teacher perception in word list evaluation .............................................. 29

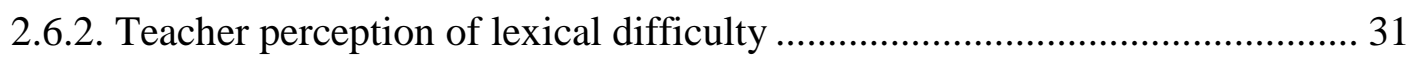

2.6.3. Teacher perception of vocabulary learning and instruction in general ......... 32

2.7. Learner proficiency in word list development ................................................... 34

2.7.1. L2 learners' vocabulary knowledge .............................................................. 34

2.7.2. How are existing word lists adaptable to learners' proficiency? .................. 38 


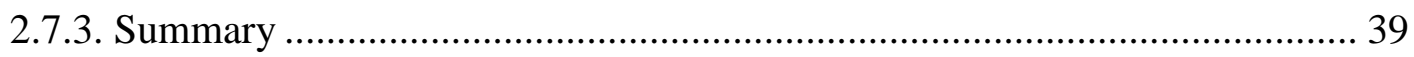

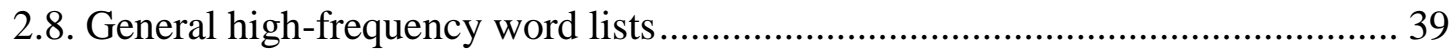

2.8.1. What are existing general high-frequency word lists? ............................... 39

2.8.2. What is the best general high-frequency word list for L2 learners? ............ 47

2.8.3. What is the value of general high-frequency words in academic spoken

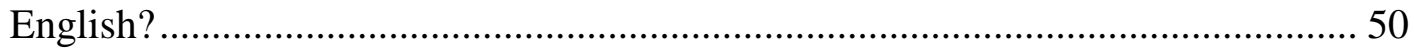

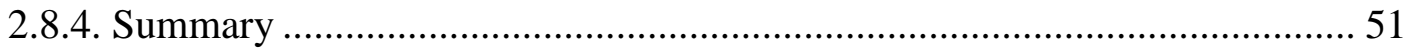

2.9. Summary of the chapter and rationale for the next chapter .............................. 52

Chapter 3 - Study 1: Lexical coverage of general high-frequency word lists ........ 57

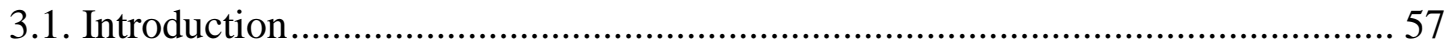

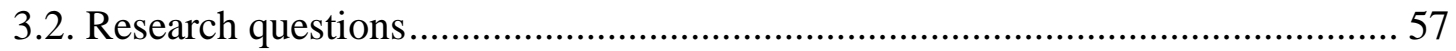

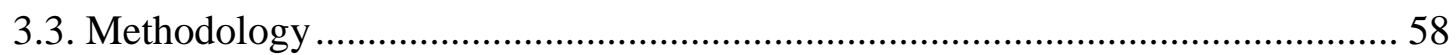

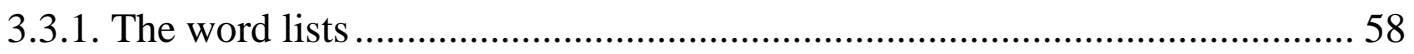

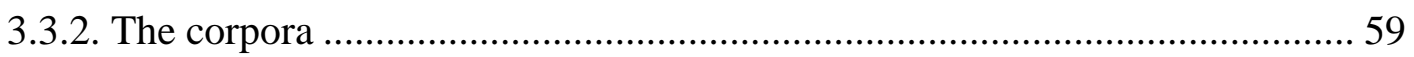

3.3.3. How to compare word lists of different units of counting ........................... 62

3.3.4. How to compare word lists with different numbers of items........................ 64

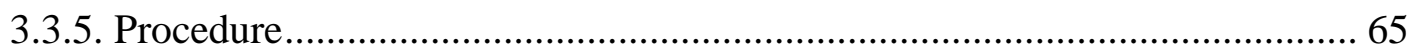

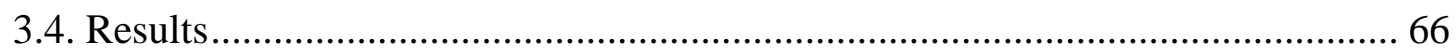

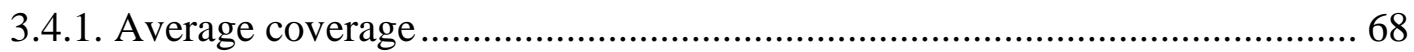

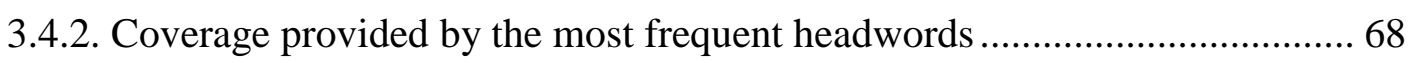

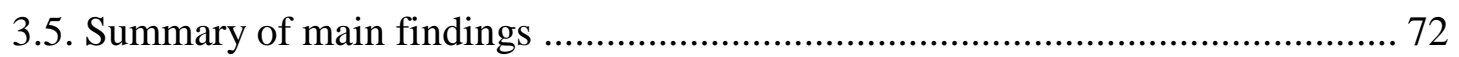

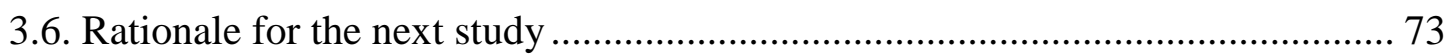

Chapter 4 - Study 2: Teacher perception of word usefulness and learner

knowledge of general high-frequency words ................................................................... 75

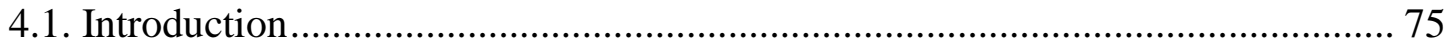

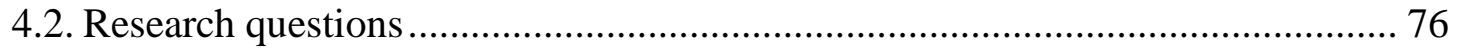

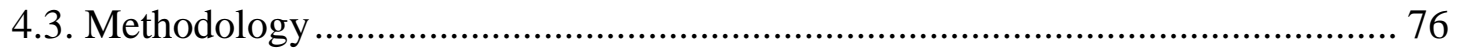

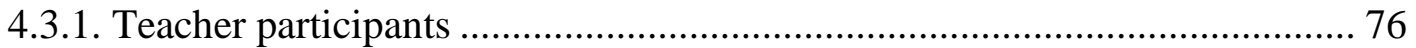

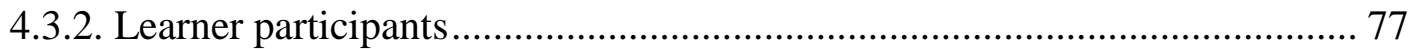

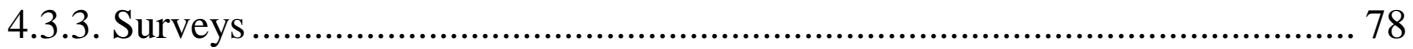

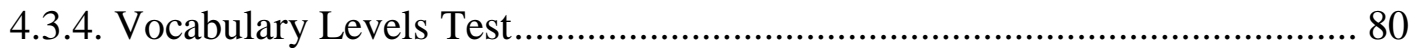

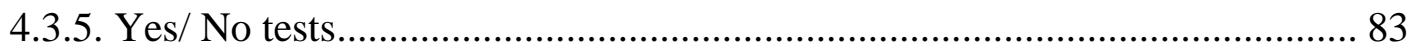

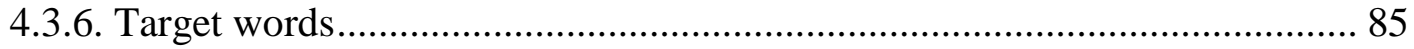

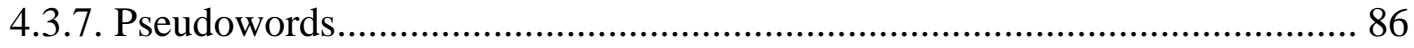

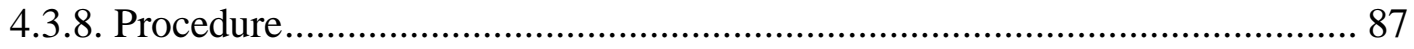

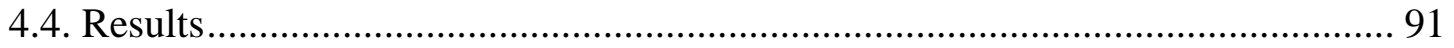

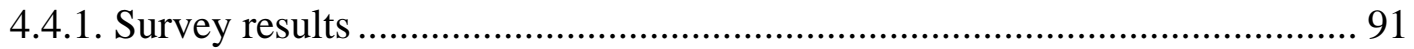




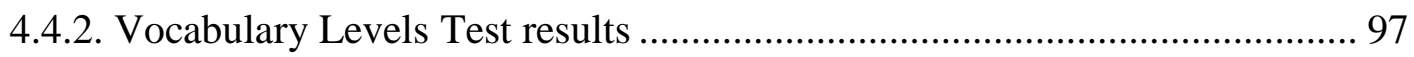

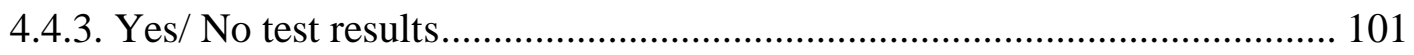

4.4.4. Relationships between the corpus, teacher, and learner data ..................... 103

4.5. Summary of main findings........................................................................... 106

4.5.1. The receptive vocabulary levels of Vietnamese EFL learners ................... 106

4.5.2. Teacher perception of word usefulness and learner knowledge of the BNC/COCA2000 and New-GSL words ........................................................ 107

4.5.3. Relationship between lexical coverage, teacher perception, and learner

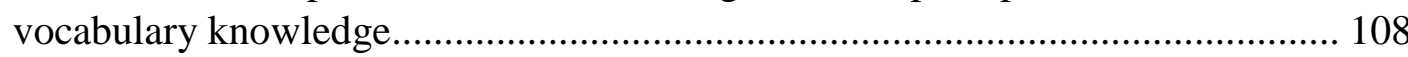

4.5.4. The correlations of three groups of teachers with the learner vocabulary

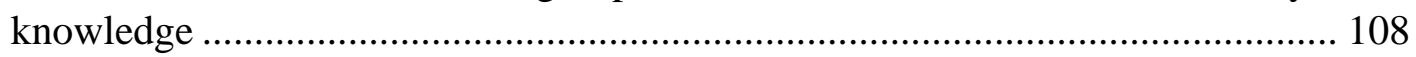

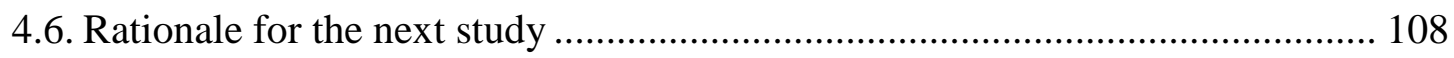

Chapter 5 - Study 3: Developing and validating an academic spoken word list.. 109

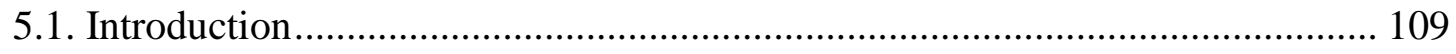

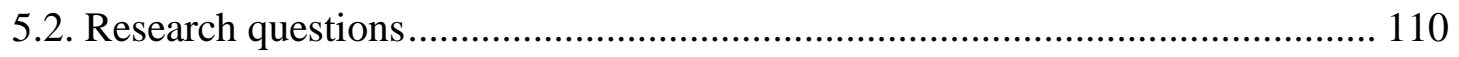

5.3. Developing the two academic spoken corpora .............................................. 110

5.3.1. Materials selection for the two academic spoken corpora ........................ 111

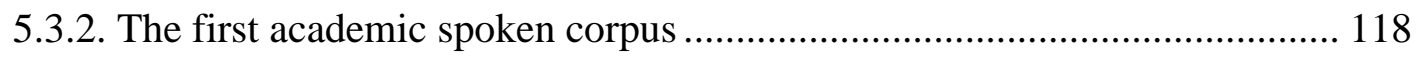

5.3.4. The second academic spoken corpus ...................................................... 124

5.4. Developing the academic written corpus and non-academic spoken corpus..... 129

5.5. Determining the unit of counting for the ASWL ............................................. 132

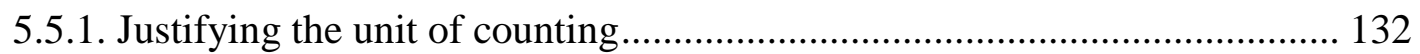

5.5.2. Identifying the word families in the first academic spoken corpus............. 133

5.5.3. Calculating the range, frequency and dispersion of the word families in the first academic spoken corpus .................................................................... 136

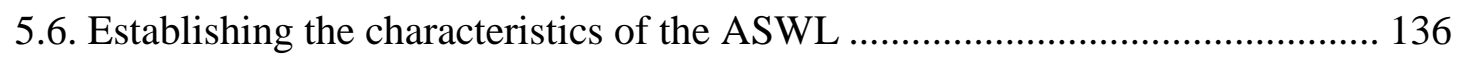

5.7. Determining the criteria for selecting the ASWL words ................................. 138

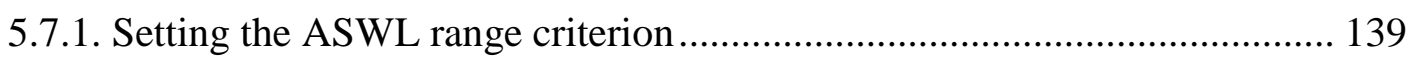

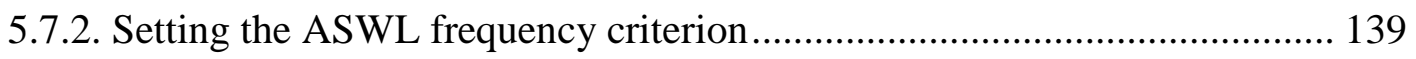

5.7.3. Setting the ASWL dispersion criterion ................................................... 144

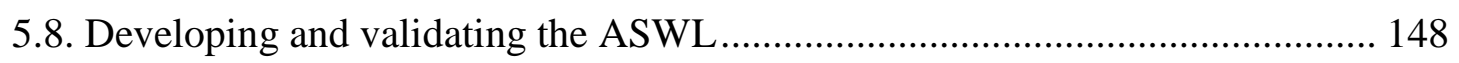

5.9. Determining the potential coverage that learners may reach by learning the

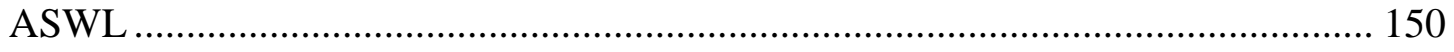

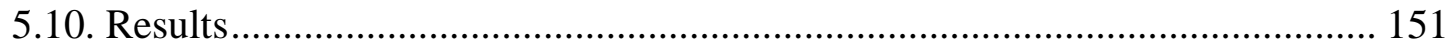

5.10.1. The most frequent, wide ranging, and evenly distributed words in academic speech

5.10.2. Coverage of the ASWL in hard-pure, hard-applied, soft-pure, and softapplied speech 
5.10.3. Coverage of the ASWL in academic speech, academic writing, and nonacademic speech

5.10.4. General high-frequency words in academic spoken, non-academic spoken

English, and written English

5.10.5. General high-frequency words and academic written words in the ASWL

5.10.6. Potential coverage of academic speech that learners may reach if they learn the ASWL

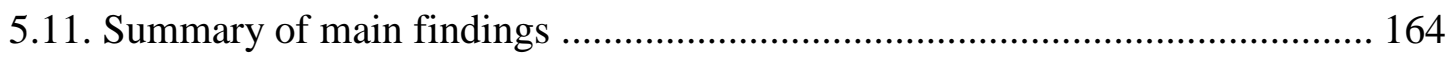

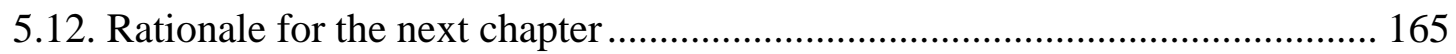

Chapter 6 - Discussion ......................................................................................... 167

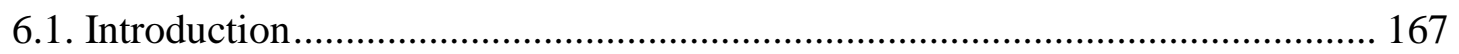

6.2. What is the importance of general high-frequency words in L2 vocabulary learning?.

6.2.1. What is the value of general high-frequency words in academic spoken English?.....

6.2.2. What is the most suitable general high-frequency word list for L2 learners?

6.2.3. How many items should be in a general high-frequency word list? ........... 171

6.3. How can word lists better support L2 vocabulary development?...................... 174

6.3.1. How can word lists be adaptable to learners' proficiency? ........................ 175

6.3.2. How can word lists be adaptable to learners' academic disciplines?.......... 177

6.3.3. What sources of information should be used in word list validation? ........ 182

6.4. How important is teachers' familiarity with learners' characteristics in L2

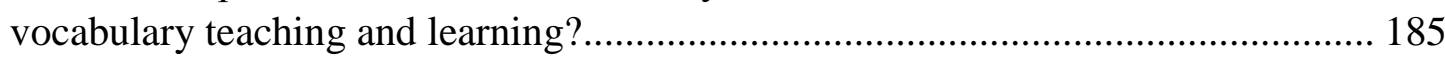

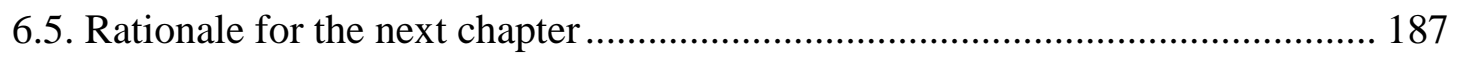

Chapter 7 - Conclusion............................................................................................. 189

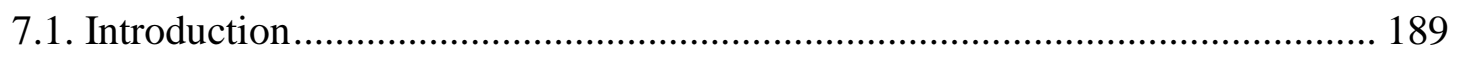

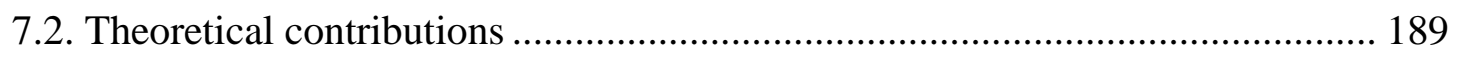

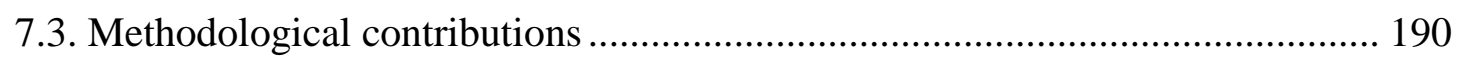

7.3.1. How to make an academic word list adaptable to learners' proficiency? ... 190

7.3.2. How to validate corpus-based word lists?.............................................. 191

7.3.3. How to compare word lists of different units of counting?........................ 193

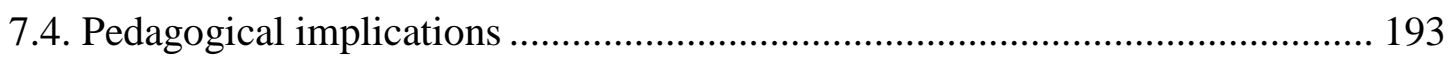

7.4.1. Developing learners' knowledge of the BNC/COCA2000 and ASWL words

7.4.2. Combining information from corpora, teachers, and learners in word list

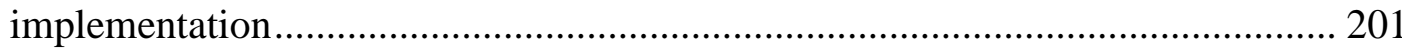

7.4.3. Enhancing teachers' knowledge of learners in vocabulary teaching .......... 202 


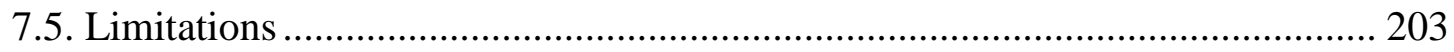

7.5.1. Limitations related to common assumptions............................................. 203

7.5.2. Limitations caused by practical reasons ................................................... 204

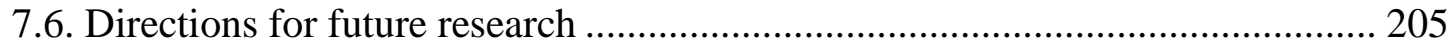

7.6.1. Further validation of general-high frequency word lists and the ASWL.... 206

7.6.2. Development of other specialized word lists .......................................... 206

7.6.3. Focusing on other aspects of academic spoken vocabulary ....................... 207

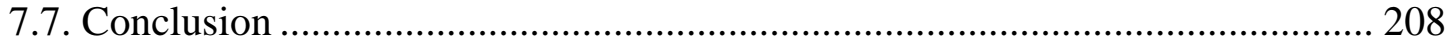

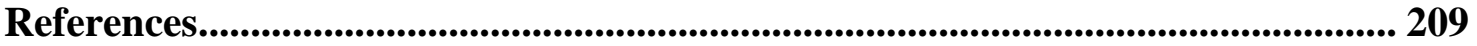

Appendices 


\section{List of Tables}

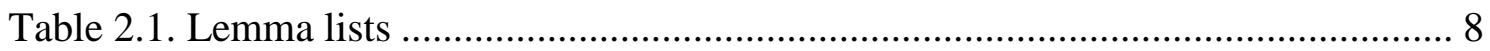

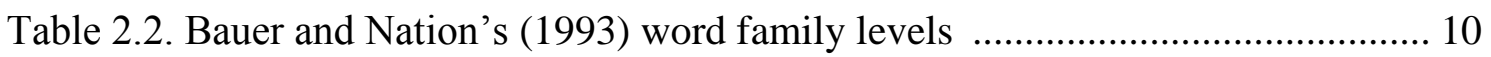

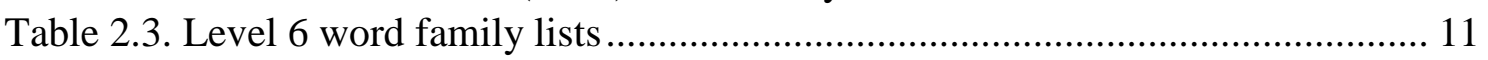

Table 2.4. Percentage of the most frequent 2,000 words known by Laufer's (1998)

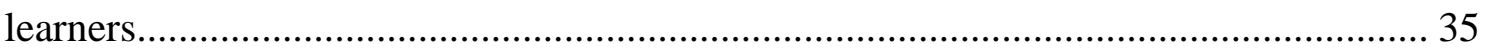

Table 2.5. Coverage of West's (1953) GSL in different text types................................ 41

Table 2.6. Coverage of Nation's (2006) BNC2000 in different text types ..................... 44

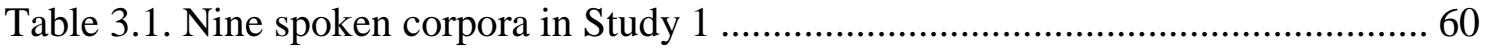

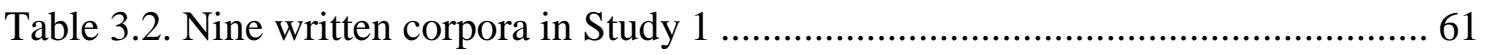

Table 3.3. Average coverage of the GSL, BNC2000, BNC/COCA2000 and New-GSL

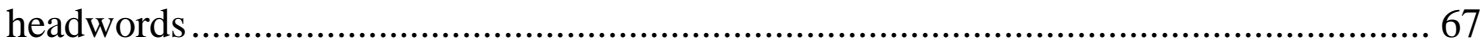

Table 3.4. Coverage by the most frequent GSL, BNC2000, BNC/COCA2000 and New-

GSL headwords

Table 3.5. Range of the coverage by the most frequent GSL, BNC2000,

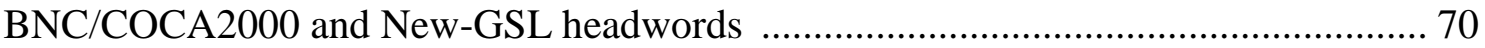

Table 3.6. Coverage by the GSL, BNC2000, BNC/COCA2000, New-GSL headwords at

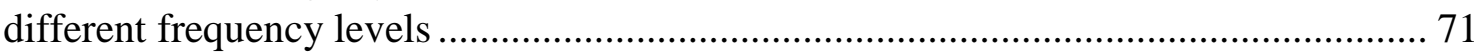

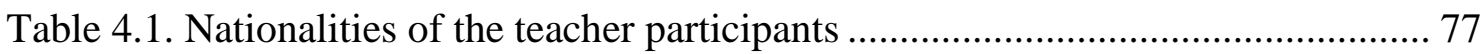

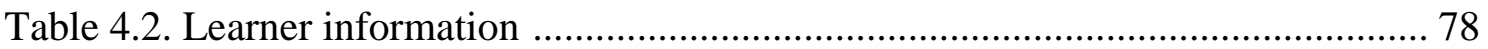

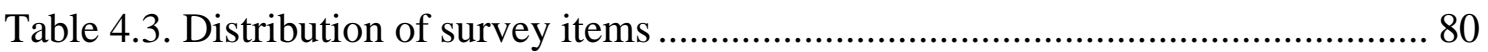

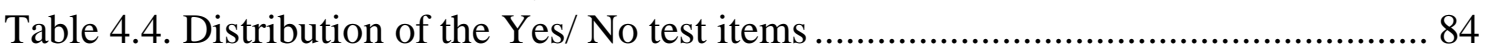

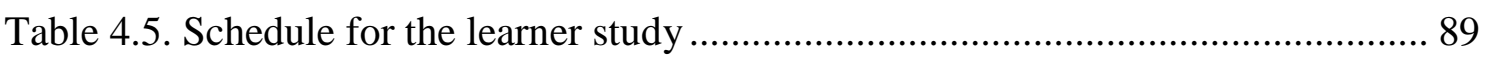

Table 4.6. Proportion of the BNC/COCA2000 and New-GSL rated as useful words by all teachers

Table 4.7. Proportion of the BNC/COCA2000 words rated as useful words by each

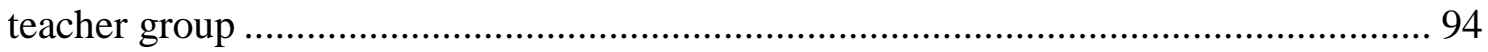

Table 4.8. Proportion of the New-GSL words rated as useful words by each teacher

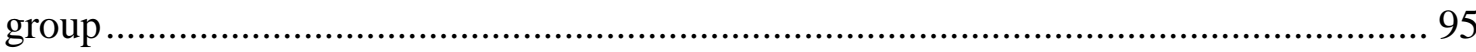

Table 4.9. Differences in the proportion of the BNC/COCA2000 and New-GSL words

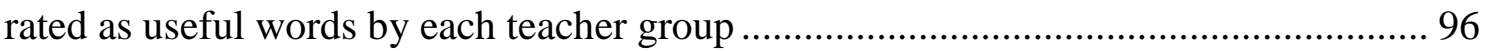

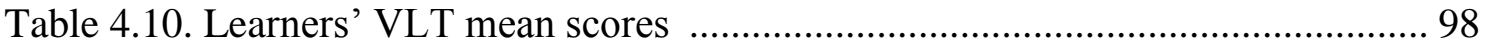

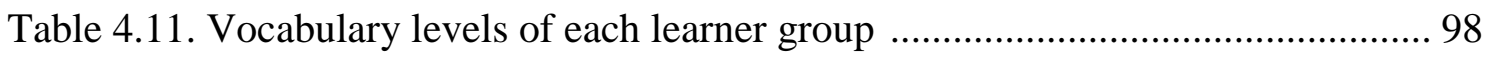

Table 4.12. Learners' VLT mean score according to academic majors ....................... 100

Table 4.13. Learners' VLT mean score according to university year .......................... 100

Table 4.14. Number of learners at different cut-off points of checked pseudowords .. 102 Table 4.15. Proportion of the BNC/COCA2000 and New-GSL words among the words

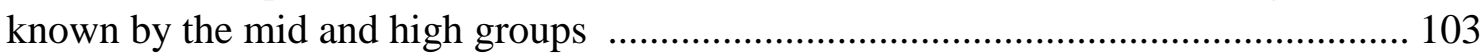

Table 4.16. Proportion of the BNC/COCA2000 and New-GSL words among the words

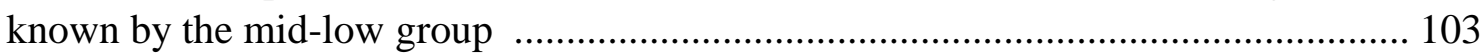

Table 4.17. Correlation between lexical coverage and teacher perception of word

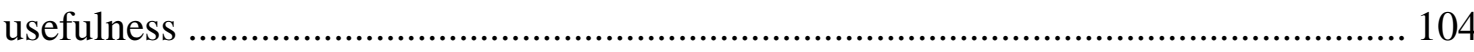

Table 4.18. Correlation between lexical coverage and learner vocabulary knowledge 105 Table 4.19. Correlation between learner vocabulary knowledge and teacher perception

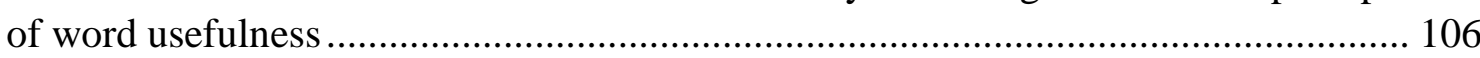

Table 5.1. Academic and non-academic corpora in Study 3 ..................................... 111

Table 5.2. Sources of the two academic spoken corpora in Study 3 .......................... 113 
Table 5.3. Speech events and disciplinary division of the corpora from publishers .... 114

Table 5.4. Speech events in the $1^{\text {st }}$ academic spoken corpus .................................... 120

Table 5.5. Composition of the $1^{\text {st }}$ academic spoken corpus ......................................... 121

Table 5.6. Composition of the hard-pure sub-corpus ( $1^{\text {st }}$ academic spoken corpus).... 122

Table 5.7. Composition of the hard-applied sub-corpus ( $1^{\text {st }}$ academic spoken corpus $) 123$

Table 5.8. Composition of the soft-pure sub-corpus ( $1^{\text {st }}$ academic spoken corpus)..... 123

Table 5.9. Composition of the soft-applied sub-corpus ( $1^{\text {st }}$ academic spoken corpus) 124

Table 5.10. Composition of the $2^{\text {nd }}$ academic spoken corpus .................................... 125

Table 5.11. Size of the four speech events in the $2^{\text {nd }}$ academic spoken corpus ............ 126

Table 5.12. Composition of the hard-applied sub-corpus ( $2^{\text {nd }}$ academic spoken corpus)

Table 5.13. Composition of the soft-pure sub-corpus ( $2^{\text {nd }}$ academic spoken corpus).. 128 Table 5.14. Composition of the soft-applied sub-corpus ( $2^{\text {nd }}$ academic spoken corpus)

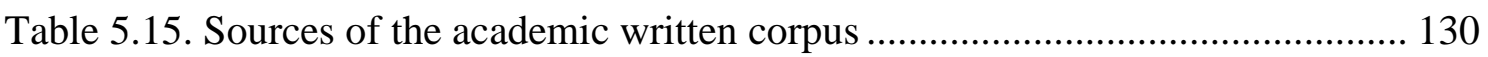

Table 5.16. Composition of the academic written corpus in terms of disciplines........ 130

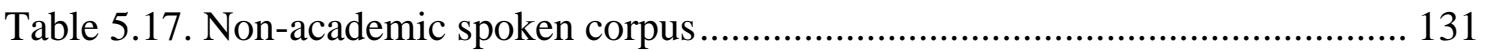

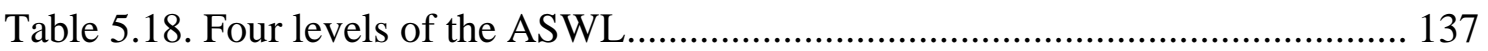

Table 5.19. Coverage of the pilot lists with frequency cut-off points from 50 to 350 in

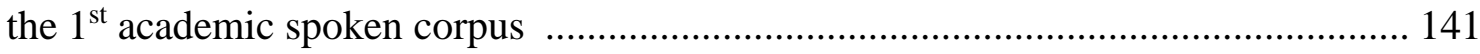

Table 5.20. Coverage of the pilot lists with frequency cut-off points of 350 and 300 in the $1^{\text {st }}$ academic spoken corpus (excluding Level 1)

Table 5.21. Difference in the coverage provided by the pilot lists with frequency cut-off points of 350 and 370 in four sub-corpora of the $1^{\text {st }}$ academic spoken corpus ............ 142 Table 5.22. Twenty-three word families beyond the most frequent 2,000 BNC/COCA word families added when the frequency cut-off point moved from 370 to 350 ........ 143

Table 5.23. Range and dispersion of the 23 word families ..................................... 143

Table 5.24. Coverage of the pilot lists with $D$ of $0.1,0.2$ and 0.6 in the $1^{\text {st }}$ academic

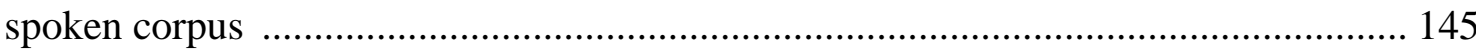

Table 5.25. Difference in the coverage in the $1^{\text {st }}$ academic spoken corpus provided by each level of the pilot lists with D of $0.3,0.4,0.5,0.6,0.7$, and Coxhead's uniformity

Table 5.26. Ranking in term of even distribution in the $1^{\text {st }}$ academic spoken corpus of the pilot lists with D of $0.3,0.4,0.5,0.6,0.7$, and Coxhead's uniformity .................... 146 Table 5.27. Distribution of the 301 additional word families across the BNC/COCA

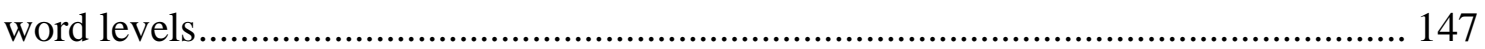

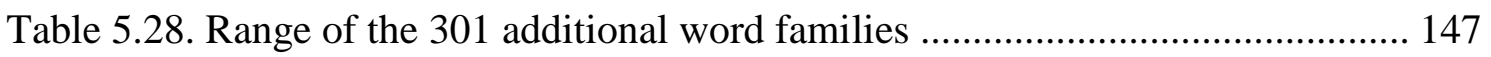

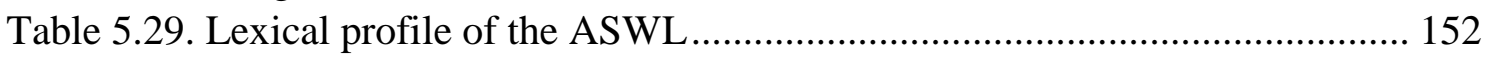

Table 5.30. Frequency of the ASWL levels in the $1^{\text {st }}$ academic spoken corpus .......... 153

Table 5.31. Range of the ASWL words in the $1^{\text {st }}$ academic spoken corpus.................. 153

Table 5.32. Range of the ASWL levels in the $1^{\text {st }}$ academic spoken corpus .................. 154

Table 5.33. Dispersion of the ASWL word in the $1^{\text {st }}$ academic spoken corpus ............ 154

Table 5.34. Dispersion of each ASWL level in the $1^{\text {st }}$ academic spoken corpus ......... 155

Table 5.35. Number of the ASWL sub-lists of function words and lexical words ...... 155

Table 5.36. Coverage of the ASWL in the sub-corpora of the two academic spoken

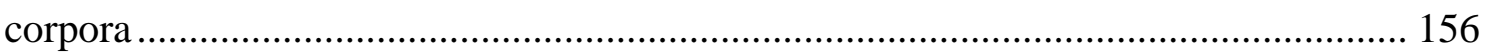

Table 5.37. Coverage of the ASWL levels in each sub-corpus .................................. 157

Table 5.38. Coverage of the ASWL in the three validating corpora ........................... 157 
Table 5.39. Coverage of the BNC/COCA2000 in the four corpora

Table 5.40. ASWL words in Nation's (2012) BNC/COCA2000, Coxhead's (2000)

AWL, and Gardner and Davies's (2014) AVL

Table 5.41. Potential coverage gained by learners with the aid of the ASWL

163

Table 5.42. Potential coverage of non-academic speech from different word lists for low and mid-low groups 


\section{List of Figures}

Figure 2.1. Nation's (2013, p.49) framework of vocabulary knowledge 14

Figure 2.2. Teacher cognition, schooling, professional education, and classroom

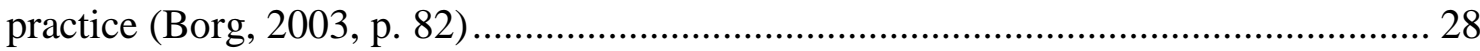

Figure 4.1. Instructions included in the surveys ....................................................... 79

Figure 4.2. Format of the VLT (Nation, 1983, 1990; Schmitt, Schmitt, \& Clapham,

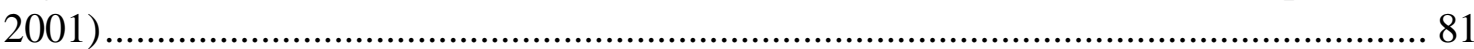

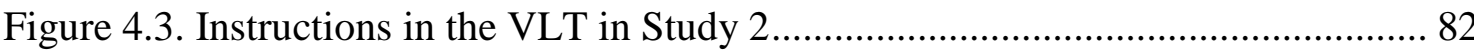

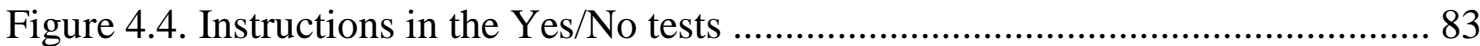

Figure 5.1. Potential coverage reached by learners of different vocabulary levels with

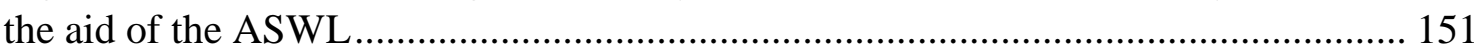

Figure 7.1. Vocabulary learning sequences for different groups of learners ................ 194

\section{List of Appendices}

Appendix 1. Sample of the learner Yes/ No tests................................235

Appendix 2. Pseudowords in Study 2 ........................................2237

Appendix 3. Ethics Approval...................................................241

Appendix 4. Amendment approval................................................. 242

Appendix 5. Teacher information sheet....................................... 243

Appendix 6. Teacher consent form............................................. 244

Appendix 7. Learner information sheet...................................... 245

Appendix 8. Learner information sheet (English translation)...................... 246

Appendix 9. Learner consent form ............................................. 247

Appendix 10. Learner consent form (English translation)..............................248

Appendix 11. Spoken materials from Yale courses...............................249

Appendix 12. Spoken materials from Massachusetts Institute of Technology courses

Appendix 13. Spoken materials from University of California, Berkeley............ 257

Appendix 14. Spoken materials from Stanford Engineering courses ...................259

Appendix 15. Written materials from Massachusetts Institute of Technology..........260

Appendix 16. Sample of 'predict' in the three ASWL versions........................ 264

Appendix 17. Sub-lists of the ASWL headwords at Level 1 .........................265

Appendix 18. Sub-lists of the ASWL headwords at Level 2 .............................273

Appendix 19. Sub-lists of the ASWL headwords at Level 3 .........................278

Appendix 20. Sub-lists of the ASWL headwords at Level 4 .......................... 283

Appendix 21. Publicly accessible corpora.................................... 285

Appendix 22. Source of open courseware and TED Talks......................... 285 


\section{List of Abbreviations}

\begin{tabular}{|l|l|}
\hline Abbreviation & Full form \\
\hline 2K & $\begin{array}{l}\text { The 2,000-word level of Schmitt, Schmit \& Clapham's (2001) } \\
\text { Vocabulary Levels Test }\end{array}$ \\
\hline The 3,000-word level of Schmitt, Schmit \& Clapham's (2001) \\
Vocabulary Levels Test
\end{tabular}




\begin{tabular}{|l|l|}
\hline Abbreviation & Full form \\
\hline MIT & Massachusetts Institute of Technology \\
\hline MW & Marginal words \\
\hline NAWL & Browne, Culligan, and Phillip's (n.d.) New Academic Word List \\
\hline New-GSL & Brezina and Gablasova's (2015) New General Service List \\
\hline NGSL & Browne's (2014) New General Service List \\
\hline NUCASE & Newcastle Corpus of Academic Spoken English \\
\hline OANC & Open American National Corpus \\
\hline PICAE & Pearson International Corpus of Academic English \\
\hline PLT & Laufer and Nation's (1999) Productive Vocabulary Levels Test \\
\hline PN & Proper nouns \\
\hline SAWL & Nesi's (2002) Spoken Academic Word List \\
\hline SBCSAE & Santa Barbara Corpus of Spoken American-English \\
\hline SD & Standard Deviation \\
\hline TED Talks & Technology, Entertainment, and Design Talks \\
\hline TESOL & Teaching English to Speakers of Other Languages \\
\hline UWL & Xue and Nation's (1984) University Word List \\
\hline VLT & $\begin{array}{l}\text { Vocabulary Levels Test (Nation, 1983, 1990; Schmitt, Schmitt, \& } \\
\text { Clapham, 2001) }\end{array}$ \\
\hline VOICE & Vienna-Oxford International Corpus of English \\
\hline WSC & Wellington Corpus of Spoken New Zealand-English \\
\hline WWC & Wellington Corpus of Written New Zealand- English \\
\hline & \\
\hline
\end{tabular}




\section{Chapter 1 - Introduction}

\subsection{Why investigate vocabulary in academic spoken English?}

English is widely used as a medium of instruction at the tertiary level in both Englishspeaking countries and non-English speaking countries (Hyland \& Shaw, 2016). The popularity of distance learning and online open courses means that people can participate in English-medium academic courses regardless of their locations (Crawford Camiciottoli \& Querol-Julián, 2016). To achieve academic success, second language (L2) learners in these programs need to comprehend not only academic written English (e.g., textbooks, journal articles, book chapters) but also academic spoken English (e.g., lectures, seminars, labs, tutorials) (Biber, 2006; Becker, 2016). Because of the close relationship between vocabulary knowledge and comprehension (Hu \& Nation, 2000; Laufer \& Ravenhorst-Kalovski, 2010; Schmitt, Jiang, \& Grabe, 2011; van Zeeland \& Schmitt, 2013), it is crucial for these learners to master the words that they are likely to encounter often in a wide range of academic written and spoken texts. A great deal of research has been done to identify the most important words in academic written English: Xue and Nation's (1984) University Word List, Coxhead's (2000) Academic Word List (AWL), Browne, Culligan, and Phillip's (n.d.) New Academic Word List, and Gardner and Davies's (2014) Academic Vocabulary List (AVL). In contrast, few attempts have been made to explore the most important lexical items in academic spoken English.

To the best of my knowledge, only two studies have developed academic spoken word lists. Nesi (2002) created a list of single words, the Spoken Academic Word List, while Simpson-Vlach and Ellis (2010) developed a list of formulas, the Academic Formulas List. Knowledge of multi-words is important for the development of fluency (Nation \& Webb, 2011; Simpson-Vlach \& Ellis, 2010). Yet, knowledge of single words provides an important basis for further vocabulary development. Unfortunately, Nesi's (2002) list is not available, and little has been written about this list. Therefore, we do not know much about the most important words in academic spoken English and their nature.

There are two reasons for the modest number of studies into academic spoken word lists. The first reason is the lack of a large and representative academic spoken corpus. Unlike written texts which can be quickly collected in large amounts by scanning or downloading texts from the Internet, speech needs to be recorded, manually transcribed, 
and coded (McCarthy \& Carter, 1997; O’Keeffe, McCarthy, \& Carter, 2007;

Thompson, 2006). Therefore, it takes much more time, effort, and resources to develop a large and representative academic spoken corpus than a written corpus. Consequently, today we have written academic corpora that contain hundreds of millions of words such as the 120-million running word academic section of the Corpus of Contemporary American English used by Gardner and Davies (2014) to develop their AVL. In contrast, the two largest publicly available academic spoken corpora, the British Academic Spoken English and Michigan Corpus of Academic Spoken English, each contain fewer than two-million running words.

Another reason for the lack of research on academic spoken word lists might be the assumption that academic written word lists such as Coxhead's (2000) AWL and Gardner and Davies's (2014) AVL are sufficient for learners to comprehend academic speech. This assumption, however, may be misleading. The nature of a word list is influenced by the nature of the corpus from which it was developed (Nation, 2016). It means that an academic written word list may not fully capture the most important words in academic speech. This argument is supported by the results of corpus-driven research (e.g., Dang \& Webb, 2014; Simpson-Vlach \& Ellis, 2010; Thompson, 2006) and lexico-grammar research (e.g., Biber 2006; Biber, Conrad, Reppen, Byrd, \& Helt, 2002) which suggested a distinctive difference between the linguistics features of academic speech and academic writing. Given the importance of understanding academic speech and the lack of research on academic spoken word lists, further research in this field is warranted.

\subsection{Why investigate general high-frequency vocabulary when examining academic spoken English?}

General high-frequency words (e.g., know, happy) are the words that appear very often in a wide range of academic and non-academic texts (Nation, 2013). These words have been widely recommended as the crucial starting point of vocabulary learning for any purposes, including academic purposes. A number of general high-frequency word lists (Brezina \& Gablasova, 2015; Browne, 2014; Nation, 2006, 2012; West, 1953) have been created to help learners acquire this important kind of vocabulary, and several attempts have been made to compare them (Brezina \& Gablasova, 2015; Browne, 2014; Gilner \& Morales, 2008; Nation, 2004; Nation \& Hwang, 1995). However, these studies compared a small number of lists in a small number of corpora which lack diversity. 
Importantly, all of them relied solely on the information from corpora. Consequently, it is still unclear which list is the most suitable starting point of vocabulary learning for L2 learners.

Moreover, although knowledge of general high-frequency words is fundamental for L2 learners' comprehension of academic spoken English, research also shows that the vocabulary levels of L2 learners are diverse. While some learners are able to master at least the most frequent 2,000 words (Laufer, 1998), others have difficulty mastering the most frequent 2,000 words (Barnard, 1961; Henriksen \& Danelund, 2015; Laufer, 1998; Nguyen \& Webb, 2016; Stæhr, 2008), and even the most frequent 1,000 words (Henriksen \& Danelund, 2015; Nurweni \& Read, 1999; Quinn, 1968; Webb \& Chang, 2012). As mentioned above, L2 learners who would like to comprehend academic spoken English come from different learning contexts. It means their vocabulary levels when starting learning items from academic word lists are diverse. Despite this situation, all existing academic word lists were created with the assumption that learners are a homogenous group which has the same starting point of vocabulary learning. To better support L2 learners' continual vocabulary development and achieve better comprehension of academic spoken English, it is essential to develop an academic word list which is adaptable to learners' proficiency.

\subsection{Why investigate corpora, teachers, and learners?}

There are two reasons for examining the information from corpora, teachers, and learners. First, with the advances in technology, there is a growing number of corpusbased word lists available. Although corpora provide a powerful way to identify and evaluate items in word lists because they represent the actual use of the language, researchers (e.g., Gardner, 2013; Nation, 2016) have recently questioned the use of corpora as the only source for word list construction. As the main agents in L2 vocabulary learning and teaching, teachers and learners may provide useful insight for the validation of corpus-based word lists. Research has suggested that there are relationships between the information from corpora, teachers, and learners. However, it is unclear how strong these relationships are. The only study that has involved both corpora, teachers and learners (Ellis, Simpson-Vlach, \& Maynard, 2008) in word list validation did not explicitly examine the correlation between the learners' data and teachers' data. Moreover, that study was based on a small corpus and a limited number of participants. Thus, it is beneficial to conduct a study that investigates the 
relationships between the information from corpora, teachers, and learners based on a large number of corpora, teachers, and learners with a greater degree of diversity.

Second, given the important role of teachers in L2 learning and teaching, it is surprising that teacher perception of vocabulary is an underexplored area in both vocabulary research and teacher cognition research. All studies that have investigated this area were based on samples of teachers which are fairly homogeneous. That is, the teachers shared the same teaching contexts with each other and with their learners. Therefore, it is unclear how teachers' perception change according to their familiarity with the learners' characteristics. Vocabulary research which involves teachers who have experienced teaching in different contexts is needed.

\subsection{Aims and scope of the present research}

To address the above mentioned gaps, the present research has been conducted with two primary aims. The first aim is to examine the role of general high-frequency words in enhancing L2 learners' comprehension of academic speech. It involves (1) determining the relationships between lexical coverage, teacher perceptions of word usefulness, and learner vocabulary knowledge of general high-frequency words, and (2) identifying the most suitable general high-frequency word list for L2 learners based on these criteria. The second aim is to explore the nature of academic spoken vocabulary. It includes (1) developing the Academic Spoken Word List (ASWL) which is adaptable to learners' proficiency and (2) examining the performance of the list in different discourse types: academic speech, academic writing, and non-academic speech.

The present study focuses on single word units rather than multi-word units for three reasons. First, as mentioned, knowledge of single words provides valuable support for the acquisition of multi-word units. Phrases in different lists of multi-word units may vary, but they share a considerable number of core single words (Adolphs \& Carter, 2013). Moreover, from a language learner's perspective, it may appear easier to learn a single unknown word than learning a phrase consisting of more than one unknown word (Coxhead, 2008). Hence, there is value in research on single word units. Second, this thesis compared four general high-frequency word lists, West's (1953) General Service List (GSL), Nation's (2006) most frequent 2,000 BNC word families (BNC2000), Nation's (2012) most frequent 2,000 BNC/COCA word families (BNC/COCA2000), and Brezina and Gablasova's (2015) New General Service List (New-GSL). They are lists of single words. Third, while there are lists of multiple words (Ellis et al., 2008; 
Simpson-Vlach \& Ellis, 2010), there is a lack of research on developing academic spoken word lists made up of single words. For these reasons, lists of multi-word units are beyond the scope of this thesis, and it only deals with single words.

\subsection{Significance of the present research}

The current research is of significance in several ways. First, considering the lack of research on academic spoken vocabulary and on comparing general high-frequency word lists based on different criteria, this research provides a significant contribution to the existing literature. It sheds light on the nature of academic spoken vocabulary as well as the role of general high-frequency words in helping L2 learners improve their comprehension of academic speech. These findings then provide grounds for further research on multiple aspects of academic spoken vocabulary such as vocabulary load, testing, incidental learning, and specialized word lists.

Second, this research identifies the most suitable general high-frequency word lists for L2 learners from the perspectives of corpus linguistics, teachers, and learners. It also involves the development and validation of an academic spoken word list. Word lists have a massive influence on multiple aspects of L2 learning and teaching practice (Nation, 2016; Nation \& Webb, 2011). Therefore, the findings from this research provide valuable information for teachers, textbook writers, material designers, curriculum developers, and test makers in setting learning goals, creating materials, learning activities, and tests that focus on the most important words at different stages of L2 vocabulary development.

Third, this study involves learners and teachers in the validation of general highfrequency word lists. Also, it creates an academic spoken word list that is adaptable to L2 learners' proficiency. In this way, this study promotes a more pedagogical approach towards developing and validating corpus-based word lists.

\subsection{Organization of the thesis}

This thesis consists of seven chapters. This introductory chapter is followed by a literature review (Chapter 2) which provides an in-depth discussion about research related to vocabulary in academic spoken English. The next three chapters (Chapters 35) are organized around three linked studies which respond to the two primary aims of the current research mentioned in Section 1.4. 
Studies 1 and 2 are conducted to deal with the first aim; that is investigating the role of general high-frequency words in helping L2 learners to comprehend academic spoken English. Study 1 compares the lexical coverage in nine spoken and nine written corpora of four well-known general high-frequency word lists: West's (1953) GSL, Nation's (2006) BNC2000, Nation's (2012) BNC/COCA2000, and Brezina and Gablasova's (2015) New-GSL. Study 2 further compares these lists by using teacher perception of word usefulness and learner vocabulary knowledge as the criteria. The findings of Study 1 are presented in Chapter 3, while those of Study 2 are described in Chapter 4.

Study 3 (Chapter 5) addresses the second aim of the research which is to explore the nature of academic spoken vocabulary. It identifies the most frequent, wide ranging, and evenly distributed words in academic speech and includes them in the ASWL. The list is divided into levels based on the best general high-frequency word list identified in Studies 1 and 2. Then, the ASWL is used as an instrument to examine the nature of vocabulary in academic speech. Each study builds upon the findings of the preceding study and is presented with separate research questions, methodology, results, summary of the main findings, and the rationale for the next study. This format allows the readers to have a clear picture of the aim and results of each study as well as the direction of the thesis.

Chapter 6 (Discussion) draws all the main findings from the three studies together under three main themes: (1) high-frequency words, (2) word list construction, and (3) the importance of teachers' familiarity with learners' characteristics in L2 vocabulary learning and teaching. The final chapter (Chapter 7) concludes the thesis by highlighting the theoretical, methodological, and pedagogical contributions, as well as limitations and direction for future research. 


\section{Chapter 2 - Literature Review}

\subsection{Introduction}

This thesis explores the nature of vocabulary in academic spoken English to help second language (L2) learners enhance their comprehension of academic speech in Englishmedium universities. Given the academic and spoken nature of academic speech, this chapter examines significant research on vocabulary in academic spoken English in relation to that in academic written English and general conversation with a focus on word list development and validation.

The chapter begins with a discussion of three key concepts in vocabulary studies: counting words (Section 2.2), aspects of vocabulary knowledge (Section 2.3), and the classification of vocabulary in a text (Section 2.4). After that, the question about the nature of vocabulary in academic spoken English is raised. Under this umbrella, previous studies into academic spoken word lists are discussed in relation to those into academic written word lists to highlight the fact that vocabulary in academic spoken English is somewhat lacking in word list research (Section 2.5). The next two sections look at the role of teacher perception (Section 2.6) and learners' proficiency (Section 2.7 ) in word list validation. Given the common assumption that learners already know general high-frequency words before studying items from academic word lists, Section 2.8 reviews research on general high-frequency word lists. The last part of the chapter (Section 2.9) points out the gaps from the literature and shows how these gaps were addressed in this thesis. Let us now look at each issue in turn.

\subsection{What is counted as a word?}

One core issue in vocabulary research is how to count a word. Different ways of counting words can influence the results of vocabulary studies (Gardner, 2007), especially word list studies (Nation, 2016). Ideally, the same unit of counting should be used by all researchers so that results of different studies can be compared (Schmitt, 2010). In reality, this issue is more complicated. Words can be counted either as single word units or multi-word units. Although kinds of multi-word units are important concepts of vocabulary research because of their great support for comprehending and producing language (Nation \& Webb, 2011), multi-word units are beyond the scope of this thesis for the reasons stated in Section 1.4. Therefore, this section only focuses on 
single word units. It has four sub-sections. The first three sub-sections in turn explain different kinds of units of counting in word list studies: tokens, types, lemmas, flemmas, and word families. The last sub-section raises the question about determining the most suitable unit of counting for a word list and factors influencing this decision.

\subsubsection{Tokens and types}

To better understand the definition of types, let us start with the definition of tokens, the simplest way of counting words. Tokens, or running words, are the number of word forms occurring in a text (Nation, 2013). Repeated word forms are counted as separate tokens. In contrast, types are the number of unrepeated word forms in a text (Nation, 2013). For example, the sentence, Counting words is difficult but it is fun, contains eight tokens but only seven types because the word form is is used twice. The word type has been chosen as the unit of counting in word lists for first language (L1) children (Carroll, Davies, \& Richman, 1971; Thorndike, 1921) and specialized vocabulary lists for L2 learners (Khani \& Tazik, 2013; Quero, 2015; Todd, 2017; Ward, 2009)

\subsubsection{Lemmas and flemmas}

A lemma is a larger unit of counting than word type. In a strict definition, a lemma is a set of word forms which have the same stem and part of speech, but are different in inflections and/or spelling (Francis \& Kučera, 1982). In other words, a lemma (allow) consists of a baseword (allow) together with its inflected forms (allows, allowed, allowed). Members of a lemma belong to the same word class (Gardner, 2007; Nation \& Webb, 2011; Schmitt, 2010). The lemma has been chosen as a unit of counting in a number of word lists (see Table 2.1).

Table 2.1. Lemma lists

\begin{tabular}{ll}
\hline Authors & Word lists \\
\hline Thorndike (1921) & Teacher's Word Book of 20,000 Words \\
Thorndike \& Lorge (1944) & Teacher's Word Book of 30,000 Words \\
Leech, Rayson, \& Wilson (2001) & British Nation Corpus word lists \\
Davies \& Gardner (2010) & Frequency Dictionary of American-English \\
Gardner \& Davies (2014) & Academic Vocabulary List \\
Brezina \& Gablasova (2015) & New-General Service List \\
Lei \& Liu (2016) & New Medical Academic Word List \\
\hline
\end{tabular}


A less strict way of counting lemmas is to include different parts of speech in the same lemma. Pinchbeck (2014) calls this unit of counting flemma with $f$ standing for family. For instance, according to the strict definition, form (verb) and form (noun) are counted as two lemmas. However, they are considered as one flemma. Flemmas were used as the unit of counting of Browne's (2014) New General Service List, Dang and Webb's (2016) Essential Word List, Nation's (2016) most frequent 3,000 BNC/COCA lists (BNC/COCA3000), and Browne, Culligan, \& Phillips's (n.d.) New Academic Word List.

\subsubsection{Word families}

A word family is the largest unit of counting. It consists of a stem together with its inflections, and closely related derivations (Nation, 2013). All word stems are free forms, not bound forms. For instance, belief and believe are counted as two separate word families. Similar to flemmas, word families do not distinguish part of speech. A question arising then is what should be included in a word family and what should not. Learners' knowledge of inflectional and derivational forms develops with their learning experience. It means what may be a suitable word family for one learner may be too demanding or too easy for other learners (Nation, 2013).

Bauer and Nation (1993) provide a useful solution to this problem. They used frequency, productivity, predictability, and regularity to establish a scale of word families. Frequency is the number of words with which an affix occurs. Productivity indicates the likelihood that the affix will be used to form new words. Predictability refers to how easy it is to determine the meaning of an affix. Affixes with more than one meaning may be less predictable than those with one meaning. Regularity deals with the degree of change to the base when an affix is added. As a result, Bauer and Nation's (1993) scale has seven levels with Level 1 consisting of single word types with no family members and Level 7 consisting of the least transparent members. The classification of an affix to a certain level is the result of a compromise between the mentioned criteria.

Table 2.2 presents the list of affixes at each word family level. It should be noted that, if a word family is defined as being at a particular level, it will include the stem together with its inflections and derivations made up of all affixes up to that level. For example, members of a word family at Level 6 would be the stem itself, and can potentially include eight members with the affixes at Level 2, 10 members with the affixes at Level 3, 11 with affixes at Level 4, 50 with affixes at Level 5, and 12 with affixes at Level 6. 
Table 2.2. Bauer and Nation's (1993) word family levels (adapted from Nation, 2016, p.27)

Please consult the print version of the thesis for access to the redacted content

As a result, a Level 6 word family can contain 92 members in total. The Level 6 word family is the most common unit of counting in word lists (see Table 2.3). 
Table 2.3. Level 6 word family lists

\begin{tabular}{ll}
\hline Authors & Word lists \\
\hline West (1953) & General Service List \\
Xue and Nation (1984) & University Word List \\
Ward (1999) & Engineering Word Lists \\
Coxhead (2000) & Academic Word List \\
Nation (2006) & BNC 14 word lists \\
Coxhead and Hirsh (2007) & EAP Science Word List \\
Konstantakis (2007) & Business Word List \\
Wang, Liang \& Ge (2008) & Medical Academic Word List \\
Martínez, Beck, and Panza (2009) & Academic word list of agriculture research articles \\
Nation (2012) & BNC/COCA 25 lists \\
Hsu (2013) & Eursing (2014) \\
Mungra and Canziani (2013) & Academic Word List for clinical case histories \\
\hline
\end{tabular}

\subsubsection{What is the most suitable unit of counting in a word list?}

Given the variation in the units of counting in word list studies, one question arising is which unit is the best. Considerable effort has been made in previous research to argue the choice of lemmas or word families (Gardner, 2007). Nation (2016) points out that word types, lemmas, flemmas, and word families are, in fact, different levels of word families in Bauer and Nation's (1993) scale. Word types can be considered as Level 1 word families, lemmas as Level 2, and flemmas as Level 2.5. In other words, word types, lemmas, flemmas, and Level 6 word families reflect different steps toward the full morphological knowledge (Level 7). A more accurate question thus should be 
which word family level is the most appropriate for a particular group of learners. This approach then lists all different units of counting in a systematic way and helps to achieve a higher degree of consistency in comparing and interpreting results of word list studies. However, it should be noted that Bauer and Nation's (1993) scale was based on usefulness and regularity, not learner knowledge. In other words, it focuses on what is useful for learners rather than what learners know. Learner morphological knowledge varies according to their L1 and L2 proficiency. Therefore, while Bauer and Nation's (1993) scale is a useful framework, it should be applied with flexibility when creating pedagogical word lists (Nation, 2016).

The level of word families should match the purpose of the word list study (Nation, 2016). Some important questions that need to be answered are who the target users of the list are, whether the list will be used for receptive or productive purposes, and whether the list examines technical words or non-technical words. To find the answer to these questions, the learning burden should be taken into account. Learning burden is the amount of effort needed to acquire a word family at a certain level (Nation, 2016). The idea behind word families is that learners may be able to see that word forms with the same stems are related to each other, and therefore, may find it easier to recognize or learn a word which is morphologically related to a known word rather than a totally unrelated word.

In terms of list users' characteristics, one problem of existing word families lists is that they are at either end of Bauer and Nation's (1993) scale (Nation, 2016). As can be seen from Sections 2.2.1 to 2.2.3, existing word lists focus either on Level 1 (word types), Level 2 (lemmas), Level 2.5 (flemmas), or Level 6 word families. Although these word lists may be relevant to a certain group of learners, they may be less applicable to others. For example, Level 6 may underestimate the morphological knowledge of adult native speakers of English (Brysbaert, Stevens, Mandera, \& Keuleers, 2016) but may overestimate the morphological knowledge of a considerable proportion of English as a Foreign Language (EFL) learners (Brown, 2013; Mochizuki \& Aizawa, 2000; Schmitt \& Meara, 1997; Schmitt \& Zimmerman, 2002; Ward \& Chuenjundaeng, 2009; Webb \& Sasao, 2013). Moreover, Ward (2009) and Ward and Chuenjundaeng (2009) found that the Thai EFL students in their studies had difficulty seeing the relationship between stems and derived forms because their L1 does not have these characteristics. Research also shows that learner knowledge of affixes increases with their vocabulary levels (Mochizuki \& Aizawa, 2000; Schmitt \& Meara, 1997; Schmitt \& Zimmerman, 2002). 
Together, these findings indicate that the suitable level of word family may vary according to the characteristics of target list users such as their L1 background or their L2 proficiency. This argument is supported by Nation (2013, 2016), Gardner (2007), and Schmitt (2010) who suggest that the unit of counting of a vocabulary study should match the characteristics of learners in that study.

In terms of list purposes, if the list is used for productive purposes, Level 1 word families (types) (Durrant, 2014; Sinclair, 1987) or Level 2 word families (lemmas) (Nation, 2016; Nation \& Webb, 2011) are more suitable because knowledge of one word form does not mean that learners are able to use other forms of this word productively (Schmitt \& Zimmerman, 2002). However, if the study investigates receptive use, word families at higher levels may be more appropriate (Nation, 2016; Nation \& Webb, 2011; Schmitt, 2010). Learners with knowledge of the base word or even one or two members of the word family may be able to recognize other members of the same word family in reading or listening. If the study deals with technical words, Level 1 word families (types) may be suitable because not all members are technical words (Chung \& Nation, 2004; Nation, 2016; Quero, 2015).

Taken together, this section shows that there is a variation in the unit of counting in previous word list studies. Despite this fact, each unit can be assigned into one of the levels in Bauer and Nation's (1993) word family scale. To determine the most suitable word family level for a word list, list developers should consider the purpose of their studies. Some important factors that should be taken into account are the characteristics of the target users and the list purposes.

\subsection{What is involved in knowing a word?}

The previous section looks at the unit of counting in word list studies. This section introduces another core issue of vocabulary research, that is, what it means to know a word. The section begins with a brief summary of different aspects of vocabulary knowledge proposed by previous research on L2 vocabulary acquisition. Then, it discusses more closely the aspects on which this thesis focuses.

Although different researchers may have different opinions about what is involved in knowing a word, it has been widely accepted that knowing a word is not just restricted to knowing the relationship between its forms and meaning. Richards (1976) listed word frequency, syntactic behavior, form and its derivations, association, limitations on use, semantic value, and conceptual meaning as different aspects of vocabulary knowledge. 
Although Richards (1976) did not mean to establish a general framework of vocabulary knowledge (Meara, 1996), others have characterized it as such and used it as the foundation to develop a more comprehensive framework (Aitchison, 1994; Laufer, 1997; McCarthy, 1990; Miller, 1999; Nation, 1990, 2013; Schmitt, 2000). Perhaps, the most thorough framework to date is that proposed by Nation (2013). As can be seen from Figure 2.1, in this framework, knowing a word involves knowing its forms (spoken form, written form, and word parts), meaning (form and meaning, concepts and referent, association) and use (grammatical functions, collocations, constraints of use).

Figure 2.1. Nation's (2013, p.49) framework of vocabulary knowledge

Please consult the print version of the thesis for access to the redacted content

Among different aspects of vocabulary knowledge, the relationship between form and meaning may be the most important. First, it provides the foundation for further development of other aspects (Gardner, 2013; Schmitt, 2008, 2010; Webb \& Chang, 2012). This claim is supported by the fact that the form and meaning relationship has been included in all previous studies of vocabulary knowledge although under different labels: semantic value (Richards, 1976), concept (Nation, 1990), meaning (Laufer, 1997), form and meaning (Nation, 2013). Also, it is the focus of the best known vocabulary tests such as the Eurocentres Vocabulary Size Test (Meara \& Buxton, 1987; Meara \& Jones, 1988, 1990), the Vocabulary Levels Test (McLean, Kramer, \& Beglar, 2015; Nation, 1983, 1990; Schmitt, Schmitt, \& Clapham, 2001; Webb, Sasao, \& 
Ballance, n.d.), and the Vocabulary Size Test (Nation \& Beglar, 2007). Second, numerous studies found that there is a close relationship between comprehension with vocabulary knowledge of the form and meaning relationship (Laufer \& RavenhorstKalovski, 2010; Qian, 1999; Schmitt, Jiang, \& Grabe, 2011; Stæhr, 2009). This suggests the importance of the form and meaning relationship on comprehension.

Each aspect of word knowledge can be broken down into receptive and productive knowledge. Following Schmitt (2010), Nation (2013) uses meaning recognition and meaning recall to refer to receptive knowledge, and form recognition and form recall to refer to productive knowledge. Receptive vocabulary use means perceiving the form of a word through listening or reading and retrieving its meaning. In contrast, productive vocabulary use means expressing its meaning though speaking and writing and retrieving and producing the relevant spoken or written form of the word. Receptive knowledge of a word does not necessarily entail productive knowledge of that words. Generally, learning and using a word receptively seem to be easier than learning and using it productively (Nation, 2013; Schmitt, 2010, Webb, 2009a, 2009b). Because this thesis focuses on word list development and validation, receptive knowledge of a word is the primary focus; however, productive knowledge is also important. To fully master a word, learners need to know it both receptively and productively.

In brief, there are different aspects involved in knowing a word receptively and productively. Among these aspects, knowledge of the form and meaning relationship is crucial. Knowing a word both receptively and productively is essential for L2 learners. The primary focus of this thesis is receptive vocabulary knowledge.

\subsection{How to classify vocabulary in academic English?}

The previous sections have introduced two keys concepts in vocabulary research (the unit of counting and aspects of vocabulary knowledge). This section discusses another key issue, the classification of vocabulary in academic English.

According to Nation (2013), there are two ways of classifying vocabulary. The first way uses frequency and range as the criteria. In this way, words are grouped into 1,000-item bands according to their frequency. Words at the $1^{\text {st }} 1,000$-word level are the most frequent and wide ranging words, while those at the $2^{\text {nd }} 1,000$ are less frequent and narrower ranging. The further the 1,000 -word levels are from the $1^{\text {st }} 1,000$-word level, the less frequent items in these levels become. Based on these levels, vocabulary can be divided into high, mid, and low frequency vocabulary. High-frequency words (e.g., 
know, sure) are those at the $1^{\text {st }}$ and $2^{\text {nd }} 1,000$-word levels. Mid-frequency words (e.g., aerial, inquisitive) are from the $3^{\text {rd }}$ to $9^{\text {th }} 1,000$-word levels. Low frequency words (e.g., perfusion, alkene) are beyond the $9^{\text {th }} 1,000$-word levels. Schmitt and Schmitt (2014) argue that the first 3,000 word families should be considered as high-frequency words. However, given that most existing general high-frequency word lists contain around 2,000 word families (see Section 2.8.1 below for these lists), Nation's (2013) classification was adopted in this thesis to define high, mid, and low frequency words. The second way uses learning purposes to categorize vocabulary into three kinds of vocabulary, including general, academic, and technical vocabulary. General vocabulary (e.g., know, happy) consists of lexical items that occur frequently in a wide range of non-academic areas. Academic vocabulary (e.g., define, equivalent) consists of lexical items that have high frequency in a wide range of academic areas, but are low in frequency in non-academic areas. Technical vocabulary consists of lexical items that occur frequently in a specific area or part of that area. For example, myocardial and edema are technical words in the field of medicine. Unlike general high-frequency vocabulary, academic words and technical words can occur across any frequency levels. This classification of vocabulary is aligned with the distinction between English for General Academic Purposes (EGAP) and English for Specific Academic Purposes (ESAP) suggested by researchers working in the field of English for Academic Purposes (EAP) (e.g., Basturkmen, 2003, 2006, 2010; Blue, 1988; Hyland, 2006). EGAP focuses on the core skills, linguistic features, and activities that are relevant for students studying any academic subject areas while the ESAP is relevant for students in certain academic subject areas.

This thesis focuses on developing an academic spoken word list for EGAP programs which consists of L2 learners from a wide range of academic subject areas. Therefore, general high-frequency words (i.e., the most frequent 2,000 words of general vocabulary from the $1^{\text {st }}$ and $2^{\text {nd }} 1,000$-word levels) and academic words are the focus of the present thesis. These words represent different kinds of vocabulary that L2 learners in these programs should focus on at different stages of their learning to achieve a solid vocabulary foundation for further acquisition of technical vocabulary. General highfrequency words are crucial for any learning purposes, including academic purposes (Nation, 2013). Learning a small number of general high-frequency words may allow learners to recognize a large proportion of different discourse types, both academic and non-academic. 
Knowledge of academic words is also important for EAP learners for three reasons. First, it helps them establish their membership in the academic community (Ivanič, 2004; McCarthy \& O’Dell, 2008; Santos, 1988). Second, vocabulary knowledge is a good predictor of academic success (Morris \& Cobb, 2004), which may then enable these learners to gain better economic opportunities and societal well-being (Corson, 1997; Ippolito, Steele, \& Samson, 2008; Jacobs, 2008; Nagy \& Townsend, 2012; Saville-Troike, 1984; Townsend, Filippini, Collins, \& Biancarosa, 2012). Third, compared with general vocabulary and technical vocabulary, academic words may be the most challenging vocabulary for EAP learners to acquire. They do not occur in general conversation and writing as frequently as general high-frequency words for these learners to acquire incidentally (Worthington \& Nation, 1996). Also, academic words are not closely related to the subject content; therefore, they are not as salient to the learners as technical words (Vidal, 2003, 2011) and are not likely to be explicitly taught by content teachers (Basturkmen \& Shackleford, 2015; Flowerdew, 1993). Hence, it is important for EAP programs to focus on academic words and create learning opportunities to enhance their learners' knowledge of academic vocabulary (Coxhead, 2000; Gardner \& Davies, 2014; Nation, 2013).

In sum, in terms of vocabulary classification, depending on the learning purposes, vocabulary can be classified into general vocabulary, academic vocabulary, and technical vocabulary. General high-frequency words and academic words are the focus of the thesis because knowledge of these words provides a firm vocabulary foundation for L2 learners' acquisition of technical words.

\subsection{The nature of vocabulary in academic spoken English}

This section discusses previous studies investigating the vocabulary in academic speech with a focus on developing and validating word lists. It consists of two sub-sections. The first sub-section reviews research on the vocabulary in academic spoken English. The second sub-section describes existing academic written word lists, firstly, to highlight the fact that academic spoken vocabulary is an underexplored area of vocabulary research compared with academic written vocabulary, and secondly, to examine the approaches towards developing academic written word lists to shed light on the way to identify items for academic spoken word lists. 


\subsubsection{Vocabulary in academic spoken English}

Academic spoken English is an underexplored area of vocabulary research. Among the very few studies that have investigated this area, only two studies focused on developing academic spoken word lists while the others looked at academic spoken English from the perspectives of incidental learning, vocabulary load, and the coverage of Coxhead's (2000) Academic Word List (AWL). Let us review each study in turn.

\subsubsection{Nesi's (2002) Spoken Academic Word List}

Nesi (2002) developed an academic spoken list of single words. Her Spoken Academic Word List (SAWL) was derived from the British Academic Spoken English (BASE) corpus which consists of 1.6-million running words. This corpus represents lectures and seminars recorded at two universities in the United Kingdom. It is divided into four disciplinary sub-corpora: Arts and Humanities (444,971 running words), Life and Medical Sciences (437,994 running words), Physical Sciences (345,585 running words), and Social Sciences (463,447 running words). To be included, the SAWL word families had to be outside Nation's most frequent 2,000 word families. They had to occur more than three times in each sub-corpus of the BASE and have high frequency in the corpus. When compared with Coxhead's (2000) AWL, a number of SAWL words that did not appear in the AWL exhibited the interactive and personal nature of spontaneous spoken discourse (e.g., please, perfect, stuff). Unfortunately, to date, there is no information about the items in Nesi's (2002) SAWL and the size of the list. Nor are there precise descriptions of the list development and validation as well as the overlapping items between the SAWL and the AWL. Hence, we can infer from Nesi's (2002) study that there might be a number of words shared among academic speech of multiple disciplines. Yet, we do not know exactly what these words are and how similar their distribution across different academic disciplines is. Neither do we know to what extent they are different from the vocabulary in academic written English.

\subsubsection{Ellis, Simpson-Vlach, and Maynard's (2008) and Simpson-Vlach and Ellis's} (2010) Academic Formula List

While Nesi (2002) looked at single word units, Ellis and colleagues focused on multiword units. These researchers developed three word lists: a spoken Academic Formulas List (AFL), a written AFL, and a core list which contains items common in both spoken and written discourse. The development and validation of these word lists are described in two studies: Ellis et al. (2008) and Simpson-Vlach and Ellis (2010). The latter study mainly focuses on using the information from corpora to select items for these lists 
while the former describes in detail how the information from learners and teachers was used to check the validity of the information from the corpora. This section reviews Simpson-Vlach and Ellis's (2010) study. Ellis et al.'s (2008) study will be discussed in Section 2.6.1 together with other studies into teacher perception in word list validation.

To develop their academic formulas lists, Simpson-Vlach and Ellis (2010) compiled four corpora: one academic spoken corpus, one academic written corpus, a nonacademic spoken corpus, and a non-academic written corpus. The two academic corpora each contained around 2.1-million running words. The academic spoken corpus included materials from the Michigan Corpus of Spoken English (MICASE), and the academic spoken section of the British National Corpus (BNC). It had five unequallysized sub-corpora: Humanities and Arts (559,912 running words), Social Sciences (710,007 running words), Physical Sciences (363,203 running words), and Nondepartmental/other (159,592 running words). The academic written corpus included materials from Hyland's (2004) research article corpus and the academic written section of the BNC. Four sub-corpora of this corpus were Humanities and Arts $(360,520$ words), Social Sciences (893,925 words), Natural Sciences/Medicine (513,586 words), and Technology and Engineering (349,838 words). The non-academic spoken corpus was the 2.9-million running word Switchboard corpus of unscripted casual telephone conversations. The non-academic written corpus consisted of 1.9-million running words. It was the combination of the Freiburg-LOB Corpus of British-English and Freiburg-Brown corpus of American-English.

The creation of the spoken word list and the written word list followed the same steps. Items in these lists had to satisfy two criteria. First, they had to be outside the formulas that occurred frequently in both their academic and non-academic corpora. Second, they had to have high frequency and wide range in the academic corpora. In particular, the spoken AFL items had to occur at the 10 tokens per million level or above in four out of five sub-corpora. The written AFL items had to have frequency of at least 10 tokens per million words in three out of four sub-corpora. The core AFL items had to have occurrences of 10 times in at least six out of nine sub-corpora. As a result, the number of formulas in the spoken AFL, written AFL, and core AFL was 979, 712, and 207 items, respectively.

A list of multi-word units has great value because knowledge of sequences of words is essential for fluent processing (Nation \& Webb, 2011; Simpson-Vlach \& Ellis, 2010). However, from the learners' viewpoints learning an unknown single word may appear 
easier than learning a phase consisting of more than one unknown single word (Coxhead, 2008). Moreover, knowledge of single words provides valuable support for the acquisition of multi-words. Phrases in different lists of multi-word units may vary, but they share a considerable number of core single words (Adolphs \& Carter, 2013). Therefore, while the list of formulas has great value, it is also important to explore the nature of single words in academic speech.

\subsubsection{Recent research on academic spoken vocabulary but beyond word list construction}

Not only are studies examining academic spoken word lists rare, those looking at other aspects of academic spoken vocabulary are also limited in number. There are eight recent studies that have investigated the vocabulary in academic spoken English in university contexts (Dang \& Webb, 2014; Hincks, 2003; Paribakht \& Webb, 2016; Simpson-Vlach \& Ellis, 2010; Thompson, 2006; Vidal, 2003, 2011; Webb \& Paribakht, 2015).

Two studies looked at the potential of academic lectures for incidental learning. Vidal (2003) examined whether academic lectures were suitable sources for incidental vocabulary learning. In her study with English for Specific Purposes (ESP) university students in Spain, Vidal (2003) found that these students learned a small but significant amount of vocabulary from academic lectures. Noticeably, they made greater gains for technical words than academic words which suggested that academic words are less salient to the students, and it is challenging for students to acquire academic words incidentally from listening to academic speech. Vidal (2011) then examined the incidental vocabulary learning of Spanish ESP learners from academic written texts and lectures and found similar results. Although the gain from academic listening was not as great as from academic reading, it was still significant. The learners made the highest gain of technical words from listening while the gain of technical words and low frequency words from reading was similar. In both cases, the learners made the lowest gains of academic words. Taken together, the results of the two studies indicate that, although academic lectures are valuable sources for L2 learners to acquire vocabulary, learners mainly attend to technical words while academic words are often neglected. This supports the argument in Section 2.4 that calls for more attention from EAP programs to focus on academic vocabulary.

Dang and Webb (2014) estimated the number of words necessary to reach adequate comprehension of lectures and seminars. Their analysis of the BASE corpus showed 
that a vocabulary size of 4,000 and 8,000 word families plus proper nouns and marginal words is needed to reach $95 \%$ and $98 \%$ coverage of academic spoken English, respectively. These vocabulary sizes are larger than the sizes needed to comprehend general spoken English: 2,000-3,000 word families (95\%) and 6,000-7,000 word families (98\%). Similar results were reported by Webb and Paribakht (2015) who examined the lexical profiles of listening passages (interviews, announcement, dialogues, and short lectures) from the CanTEST, a university admission test in Canada. They found that knowledge of 4,000 word families is needed to reach $95 \%$ coverage of academic spoken text while knowledge of 10,000 word families is necessary to reach $98 \%$ coverage. Compared with a vocabulary size of 3,000 word families and 6,0007,000 word families to reach $95 \%$ and $98 \%$ coverage of general spoken English (Nation, 2006; Webb \& Rodgers, 2009a, 2009b), the findings of Dang and Webb (2014) and Webb and Paribakht (2015) indicate that learners needs more vocabulary to comprehend academic spoken English than to comprehend general spoken English. In other words, knowledge of general high-frequency words is not enough for L2 learners to achieve adequate comprehension of academic spoken English.

Dang and Webb (2014) also examined the coverage of Coxhead's (2000) AWL in academic speech from four disciplinary sub-corpora of the BASE corpus: Arts and Humanities, Life and Medical Sciences, Physical Sciences, and Social Sciences. They found that the variation in the coverage of the AWL between these sub-corpora was not as great as that reported in studies which examined the coverage of the list in academic written texts (Cobb \& Horst, 2004; Coxhead, 2000; Hyland \& Tse, 2007). This finding indicates that the similarities between the vocabulary in academic spoken English are greater than the differences.

However, Dang and Webb's (2014) results also revealed that the AWL accounted for only $4.41 \%$ of the BASE corpus, which is much lower than the $10 \%$ figure reported in academic written texts. Similar results were reported by other studies which investigated the coverage of Coxhead's (2000) AWL in different kinds of academic speech. The AWL provided only $2.4 \%$ coverage of student presentations (Hincks, 2003), $4.9 \%$ coverage of lectures (Thompson, 2006), and $6.48 \%$ coverage of university admission listening comprehension tests (Paribakht \& Webb, 2016). Likewise, Simpson-Vlach and Ellis (2010) found that only 207 out of 712 items (29.07\%) in their written AFL appeared in their spoken AFL. These findings suggest that perhaps vocabulary in academic speech is distinctive from that in academic writing. This 
assumption is supported by the results of lexico-grammar research which used Biber's (1988, 2006) multidimensional framework.

Biber, Conrad, Reppen, Byrd, and Helt (2002) and Biber (2006) analyzed the linguistic features of a range of academic speech and writing and reported a clear-cut distinction between the linguistic features of the two modalities. That is, academic speech had a higher degree of interaction and personal involvement while academic writing had a higher density of information.

Csomay (2006) compared the linguistic features of university classroom teaching with those of face-to-face conversation and academic writing. She found that the language in university classroom teaching is more similar to that of academic writing than to faceto-face conversation in terms of informational focus and abstract style. However, the linguistic features of university classroom teaching are more similar to those in face-to face conversation than to academic writing in terms of involved production and explicit reference. Csomay (2006) also reported that, apart from these similarities, academic spoken language is distinctive from the other two registers in term of on-line informational elaboration. This feature characterizes the situation where the speakers share the same contexts with the listeners, and are under the pressure of transferring the information (informational) in real time production conditions (on-line).

The differences between vocabulary in academic spoken and written English are understandable given that the nature of a word list is influenced by the nature of the corpus from which it has been developed (Nation, 2016; Schmitt, 2010). In other words, previous studies suggest that the vocabulary in academic speech is distinctive from that of academic writing. Therefore, a word list developed specifically from an academic spoken corpus may capture the language in academic speech more fully than a word list which is developed from an academic written corpus or a corpus with mainly academic written materials.

Taken as a whole, this section has shown that few studies have investigated the nature of vocabulary in academic spoken English. Research that particularly focused on developing an academic spoken word list is even rarer. To further demonstrate this fact, the next section reviews research on academic written word lists. Another purpose of the next section is to examine the methods that have been used to develop academic written word lists. This will shed light on the best way to determine items for academic spoken word lists. 


\subsubsection{Vocabulary in academic written English}

Compared with vocabulary in academic spoken English, vocabulary in academic written English is a well-established area of vocabulary research. It can be illustrated by the large number of academic written word lists. To date, Coxhead's (2000) AWL is the most influential academic written word list. However, identifying the most important words in academic written English has been a continual interest of many researchers before and after the development of the AWL. This section reviews these word lists in turn.

\subsubsection{Lists before Coxhead's (2000) Academic Word List}

Creating lists of important words for academic study has drawn attention from researchers since the early 1970s. These lists were developed with different approaches from counting word frequency and range in corpora of academic texts (Campion \& Elley, 1971; Praninskas, 1972) to investigating learners' vocabulary difficulties (Ghadessy, 1979; Lynn, 1973). Campion and Elley (1971) created a list of 3,200 words from a 301,800 running word corpus of university textbooks, lectures, and examination papers. Praninskas's (1972) list consists of 507 words selected from a 272,466 running word corpus of university textbooks. Both studies assumed that general service vocabulary had been known by the learners for whom the lists were developed.

Therefore, Campion and Elley excluded Thorndike and Lorge's (1944) first 5,000 word types from their list while Praninskas excluded West's (1953) General Service List (GSL) words from her list.

Lynn (1973) and Ghadessy (1979) took a different approach. They suggested that course materials should focus on lexical items that learners find difficult. Therefore, Lynn (1973) created a list of 120 words selected from the 10,000 annotations written by first-year university students in their books and cyclo-styled handouts. Ghadessy (1979) analyzed the words noted down with translation by first-year university students in a 478,700 running word corpus of textbooks and developed a list of 795 words.

Despite their different approaches, these four academic written word lists were all conducted on a small scale and based on very small hand-compiled corpora (Coxhead, 2000). To deal with this limitation, Xue and Nation (1984) combined the four academic word lists into the University Word List (UWL), which consists of 836 word families. Similar to Praninskas's (1972) lists, the UWL includes words with high frequency in a range of academic texts that are outside West's (1953) GSL. The specialized nature of the UWL was validated by its high coverage in academic texts and low coverage in non- 
academic texts. Its coverage in academic written texts was $8.4 \%$ (Sutarsyah, Nation, \& Kennedy, 1994) and 9.8\% (Coxhead, 2000). In contrast, it provided only 3.9\% coverage of newspapers (Hwang, 1989) and 1.7\% coverage of fiction (Hwang, 1989). Because of its validity, the UWL was widely used for a long time (Coxhead, 2000). However, the UWL was not really corpus-based. Only two of the lists from which the UWL was derived were based on corpora (Campion \& Elley, 1971; Praninskas, 1972). Consequently, the UWL lacked consistent selection principles (Coxhead, 2000) and included the limitations of the component lists (Coxhead, 2000; Nation \& Webb, 2011).

\subsubsection{Coxhead's (2000) Academic Word List}

To date, perhaps Coxhead's (2000) AWL is the best-known academic written word list. The AWL contains 570 word families which were selected from an academic written corpus of 3.5-million running words. The corpus was divided into four sub-corpora: arts, commerce, law, and science. Each sub-corpus represents materials from seven subjects. Each subject contained around 875,000 running words.

Similar to Campion and Elley (1971), Praninskas (1972) and Xue and Nation (1984), Coxhead (2000) assumed that the target users of her list already knew general highfrequency words. Therefore, she looked for items with high frequencies, wide ranges, and even distribution in her academic corpus that were not general high-frequency words. To be selected, the AWL word families must satisfy four criteria. In terms of specialized occurrence, the selected word families must be outside West's (1953) GSL words. In terms of range, the AWL word families had to occur in all four sub-corpora, and in at least half of the subject areas. In terms of frequency, the AWL word families had to occur at least 100 times in the whole academic corpus. Range was considered before frequency to minimize the chance that high-frequency but narrow-ranging word families would be included in the AWL. In terms of uniformity, or dispersion, the selected word families had to occur at least 10 times in each sub-corpora, which ensured that the selected word families would not be biased toward a certain subject.

Although the AWL has fewer items, it provided higher coverage (10\%) than the UWL $(9.8 \%)$ in the academic written corpus. Moreover, its coverage in each sub-corpus ranged from $9.1 \%$ to $12.0 \%$. The specialized nature of the AWL is confirmed by its high coverage in an independent academic written corpus (8.5\%) and low coverage in a corpus of fiction $(1.4 \%)$. 
The strength of the AWL over the preceding lists is the comprehensive principles that guided its development and validation. First, the AWL was developed from a larger, more balanced, and representative corpus than those used to create previous word lists. The AWL corpus met Sinclair's (1991) criterion of corpus size; that is, a corpus should have millions of running words. Moreover, it included materials from a wide range of academic disciplines and sources (university textbooks, articles, book chapters, and laboratory manuals). It had a good balance in the number of subjects, words, and texts of different lengths in each sub-corpus. Consequently, the corpus represented an accurate picture of academic vocabulary that first-year university students may encounter in their academic reading. The second strength of the AWL is the thorough criteria used to select the AWL words. This ensured that the AWL would capture lexical items outside general high-frequency words occurring frequently in a wide range of academic disciplines. Third, the validation step of the AWL is innovative. A word list is only valid if it is evaluated in an independent corpus or compared with other lists (Nation \& Webb, 2011). Examining the coverage of the AWL in two independent corpora (an academic corpus and a non-academic corpus) ensures that the AWL is a truly academic word list.

The validity of the AWL and its well-established principles have been confirmed by its consistent coverage (around 10\%) in a wide range of academic written texts (see Coxhead, 2011 for a detailed review). Moreover, intervention studies (e.g., Lesaux, Kieffer, Faller, \& Kelley, 2010; Townsend \& Collins, 2009) showed that the AWL helps L2 learners improve their comprehension of academic written texts and academic achievement. Research on EAP teachers' practice, attitude and belief of the AWL also indicates that this list is a useful tool for learners from a wide range of subjects (Banister, 2016). As a result, the AWL has been widely used to design EAP teaching materials, vocabulary tests, and dictionaries (see Coxhead, 2011, 2016 for a detailed review).

Despite its validity and great impact on L2 vocabulary learning and teaching, the AWL is not without limitations. Its biggest limitation is the use of West's (1953) GSL as the general high-frequency vocabulary baseline. As will be discussed later in Section 2.8.1.1, the GSL was developed from 1930s texts, and may not accurately reflect current vocabulary (Coxhead, 1998, 2000; Gardner \& Davies, 2014; Nation \& Webb, 2011; Richards, 1974). Therefore, using the GSL to represent general high-frequency words means that the AWL contains a number of more current general high-frequency words 
that the GSL failed to capture. This argument is supported by Nation (2004), Cobb (2010), Neufeld, Hancioglu, and Eldridge (2011), and Dang and Webb (2014), who found a considerable number of AWL words appearing in recently-created highfrequency word lists. Subsequent studies have dealt with this limitation of the AWL in different ways. These studies are presented in turn below.

\subsubsection{Browne, Culligan, and Phillip's (n.d.) New Academic Word List}

Browne et al. (n.d.) followed Coxhead's (2000) approach by creating an academic word list on the top of Browne's (2014) New General Service List (NGSL), a more current general high-frequency word list (see Section 2.8.1.4 below for a detailed description of the NGSL). Their New Academic Word List (NAWL) consisted of 963 lemmas (Level 2 word families) selected from a 288-million running word corpus. Unlike the corpora used to develop other academic written word lists, the corpus from which the NAWL was derived consisted of $98.9 \%$ written texts and $1.1 \%$ spoken texts. The written texts represented academic journals, non-fiction, and student essays in the Cambridge English Corpus. The spoken texts were taken from the BASE and MICASE corpora.

Although Browne et al. (n.d.) claimed that frequency, dispersion, and appropriateness were used as the criteria for word selection, little has been written about the method from which the NAWL was developed. Similar to the AWL, the strength of the NAWL is that it takes learners' existing knowledge of general vocabulary into account, and enables learners and teachers to avoid repeatedly learning and teaching known items. However, like other academic word lists which followed this approach, the NAWL is still affected by the nature of the general high-frequency word list on which it was built.

\subsubsection{Gardner and Davies's (2014) Academic Vocabulary List}

Gardner and Davies (2014) took a different approach by not assuming that learners already know general high-frequency vocabulary. In the development of their Academic Vocabulary List (AVL), Gardner and Davies (2014) did not rely on any ready-made lists to distinguish general high-frequency words from academic words. Instead, they used statistical power to select items that have wider range and higher frequency in academic than non-academic texts for their list. Their academic written corpus was the 120-million running word academic section of the Corpus of Contemporary AmericanEnglish (COCA) corpus. It has nine unequally-sized sub-corpora: (1) education, (2) humanities, (3) history, (4) social science, (5) philosophy, religion, psychology, (6) law and political science, (7) science and technology, (8) medicine and health, and (9) business and finance. The size of these sub-corpora ranged from $8,030,324$ to 
$22,777,656$ running words. The corpora represented three kinds of academic texts: journal articles, newspapers, and magazines. Journal articles accounted for $70.83 \%$ of the corpus.

Similar to Browne et al. (n.d.), Gardner and Davies (2014) chose lemmas (Level 2 word families) as the unit of counting for their list. To be selected, an AVL lemma had to meet the ratio, range, dispersion, and discipline measure criteria. In terms of ratio, the frequency of the lemma in the whole academic corpus had to be at least $50 \%$ higher than that in the non-academic corpus. In terms of range, the selected items had to appear with at least $20 \%$ of the expected frequency in seven or more subjects. In terms of dispersion, the selected words had to have a Juilland and Chang-Rodríguez's (1964) dispersion of at least 0.8 . In terms of discipline measure criterion, the AVL words had to occur no more than three times the expected frequency in any of the nine subject areas. As a result, the AVL contains around 3,000 lemmas (Level 2 word families) or 1,991 Level 6 word families. When the most frequent 570 AVL word families were tested in the academic, newspaper, and fiction sections of the COCA and BNC corpora, they provided the highest coverage in the academic section, then the newspaper, and the fiction sections. This indicated the validity of the AVL as an academic word list. Durrant (2016) examined the coverage of the AVL in the 2,429 pieces of university student writing from the British Academic Written English corpus. He found that the list covered an average of $34 \%$ of the words in each text of the corpus, which indicates that the list is a useful resource for EAP learners.

The strength of Gardner and Davies's (2014) approach is that the AVL was created from scratch, and therefore, was not affected by the limitation related to the general high-frequency word lists. However, creating an academic word list from scratch without considering learners' existing levels of general vocabulary may carry the risk of repeatedly teaching and learning known items, which results in inefficient learning and teaching time.

In brief, compared with the modest number of academic spoken word lists, the number of academic written word lists is impressive. Different approaches have been taken to identify the lexical items for these word lists. Despite this difference, all studies suggest that there is a considerable amount of shared vocabulary between academic writing of different subject areas, and it covers a reasonable proportion of words in academic writing. 


\subsubsection{Summary}

This section has reviewed research on academic spoken vocabulary in relation to that on academic written vocabulary. Compared with the vocabulary in academic written English, the vocabulary in academic spoken English is still an underexplored area of vocabulary research. Only one study has attempted to develop an academic spoken word list of single words, and little is written about this list. Considering the differences between the linguistic features of academic spoken and written English, further research on creating an academic spoken word list is needed. Such research will provide better insight into the nature of vocabulary in academic spoken English. In the next two sections of this chapter, we will examine the role of teacher perception and learners' proficiency in word list validation.

\subsection{Teacher perception in word list validation}

Teachers can provide useful information for word list evaluation, because they have a significant impact on how vocabulary is taught and learned (Dobinson, 2001; Joe, 2006; Leeke \& Shaw, 2000; Moir, 1996; Schmitt, 2008; West, 1953). According to Borg (2003, 2006), what teachers do in classes is influenced by their perception or cognition (i.e. what they think, believe, and know), which is, in turn, affected by many factors.

Figure 2.2. Teacher cognition, schooling, professional education, and classroom practice (Borg, 2003, p. 82)

Please consult the print version of the thesis for access to the redacted content 
As demonstrated in Figure 2.2, these factors can be looked at from three perspectives:

(1) teacher cognition and prior language learning experience, (2) teacher cognition and teacher education, and (3) teacher cognition and classroom practice. Teachers' experience as learners establishes their cognition about learning and language learning. It then shapes the basis of their initial conceptualization of L2 teaching during teacher education and may continue to have an impact on teaching practice throughout their professional lives. Teacher education also influences teacher cognition, but the impact depends on each individual teacher. In terms of the relationship between teacher cognition and classroom practice, they are mutually informing, with contextual factors playing a significant part in determining the degree of congruency between teacher cognition and their actual teaching practice. The contextual factors either make teachers change their cognition or alter their teaching practice directly without changing the cognition underlying it.

Despite the importance of teachers in L2 vocabulary learning and teaching, research on teacher cognition in vocabulary learning is fairly limited. Borg $(2003,2006)$ reviewed a range of teacher cognition studies in first, second, and foreign language contexts published up to 2006. He found that these studies focused either on generic cognition (i.e. the nature and process of teacher cognition regardless of the curricular areas) or cognition in relation to specific curricular areas. Among studies which examined teacher cognition in relation to specific curricular areas, the majority focused on grammar, reading, and writing. Vocabulary, however, is an underexplored field of teacher cognition research. Borg's (2015) latest language teacher cognition bibliography shows that this remains the case today. Word list research which used teacher cognition as a criterion is even rarer. To the best of my knowledge, only 13 teacher cognition studies that have focused on the field of vocabulary. Of these studies, only Ellis et al. (2008) and Banister (2016) used teacher perception in word list evaluation. In this section, we will focus on these two studies first and then look at other studies into teacher cognition in the field of vocabulary.

\subsubsection{Teacher perception in word list evaluation}

As mentioned in Section 2.5.1.2 above, Ellis et al.'s (2008) study is part of a larger project conducted by Ellis and colleagues to develop and validate the core AFL which contains the core formulas in academic speech and writing for EAP learners. In their study, Ellis et al.'s (2008) examined teacher perception of the instructional value of 108 items randomly selected from the core AFL (see Section 2.5.1.2 above for the detailed 
description of the AFL). These items were used as the target formulas in a series of experiments with experienced EAP teachers and language testers, and advanced L2 learners in an EAP program in the United States. The teachers and test takers judged the instructional value of the target formulas while the learners took part in a series of experiments in which their processing of the target formulas was measured. Both the teacher and learner studies suggested that teacher perception and learner knowledge significantly correlated with the information from corpora. These findings are valuable because they confirm the relationship between the information from corpora with those from teachers and learners. However, a few points should be noted about this study.

First, Ellis et al.'s (2008) study has several limitations. The number of participants was limited (20 teachers and 6-18 learners). Moreover, the academic spoken corpus from which the AFL items were selected was small (2.1-million running words) and unbalanced (the size of each sub-corpus ranging from 159,592 words to 559,912 words. Importantly, they did not examine the correlation between the learners' data and teachers' data. Therefore, what is less transparent from their study is how close the relationship between the information from learners and teachers compared with those between each source of information with the information from corpora.

Second, for the purpose of their study, the teacher participants in Ellis et al.' (2008) study shared the same context with each other and with the learner participants, and the learners were at advanced levels. Therefore, studies that compared the perception of teachers who experience teaching in different contexts may provide more insight into the nature of teacher perception on the instructional value of a word. Third, Ellis et al. (2008) focused on academic multi-words. Further research on general high-frequency words and single words is also useful.

While Ellis et al. (2008) focused on teacher perception of each individual word in a list, Banister (2016) explored teacher perception of the list as a whole. He delivered online surveys and follow-up interviews with teachers of academic English to examine their teaching practice with reference to Coxhead's (2000) AWL as well as their beliefs and attitudes towards the pedagogical value of this list for vocabulary teaching and learning. There were 193 teachers responding to the survey. A number of them were selected for interviews. Unfortunately, the number of teachers participating in the interviews is unclear from Banister's (2016) article. The results show that the AWL was widely used by the teachers, but the practice varied according to contexts. The majority of the teachers believed that the AWL was a useful tool because it could benefit a wide range 
of learners. These findings are meaningful in two ways. First, they highlight the value of universal academic word lists such as the AWL. Second, they suggest that the information from corpora and teachers is related. However, like Ellis et al.'s (2008) teachers, most of the teachers in Banister's (2016) study came from a similar teaching context; that is, $70 \%$ of them were UK-based. Although there were a proportion of teachers working in different contexts, Banister (2016) did not report any differences in the teaching practice, beliefs, and attitudes of teachers in different contexts.

Apart from Ellis et al.'s (2008) and Banister's (2016) research, most teacher cognition studies in the field of vocabulary do not particularly focus on word list validation. The next two sections describe studies into teacher perception of vocabulary.

\subsubsection{Teacher perception of lexical difficulty}

There are three studies that examined teacher perception of lexical difficulty (Brutten, 1981; McNeill, 2005; Smith \& Tajino, 2003). Brutten (1981) and McNeill (2005) investigated the relationship between teacher perception of lexical difficulty and learner vocabulary knowledge. In both studies, lexical difficulty means the words that may cause problems for learners in reading comprehension. Brutten's (1981) study was conducted with 35 L2 learners and 12 English as a Second Language (ESL) teachers in the United States. These participants were given a reading passage. The learners underlined the words in the text that hindered their comprehension while the teachers underscored the words that they thought would cause difficulty for the learners in comprehending the passage. The results revealed a significant correlation between teachers' perception of lexical difficulty and learners' vocabulary knowledge.

McNeill (2005) conducted a similar study in Hong Kong with 200 Cantonese L1 secondary school students and two groups of ESL teachers (35 Cantonese L1 teachers and 30 English L1 teachers). In this study, the participants read a text. The students completed two vocabulary tests in which they had to translate into their L1 40 English words, which were the most difficult lexical items in a text. The teachers identified the words that they thought were difficult for the learners in terms of reading comprehension. Unlike Brutten (1981), McNeill (2005) only found a significant correlation between the ratings of the Cantonese L1 teachers with learners' scores. There were no significant correlations between the ratings of the English L1 teachers with the learners' score. These results were consistent whether the teachers were experienced or inexperienced teachers. Based on these findings, McNeill (2005) concluded that ESL teachers who share the same L1 with their students are likely to 
identify the words that hinder their students' comprehension more accurately than teachers who do not. Despite the slight inconsistency, both Brutten's (1981) and McNeill's (2005) studies suggested that, in general, teachers are able to predict their learners' lexical difficulty in terms of reading comprehension.

Smith and Tajino (2003) took a different approach. They looked at teacher perception of lexical difficulty in relation to word frequency. Additionally, in their study, lexical difficulty was interpreted as the words that may be difficult for learners to acquire. In particular, Smith and Tajino (2003) asked 41 Japanese upper secondary school English as a Foreign Language (EFL) teachers to rate the difficulty levels of five words from the 2,000-word level and five words from the 5,000-word level in Schmitt et al.'s (2001) Vocabulary Levels Test (VLT). Their results showed that, although the teachers' ratings were consistent, their reasons for word selection varied, and frequency was an important factor. This finding illustrates the fact that teacher perception is influenced by many factors.

Although Brutten's (1981), Smith and Tajino's (2003), and McNeill's (2005) studies provide valuable information for the use of teacher perception in word list validation, these studies shared the same limitation with Ellis et al.'s (2008) study. That is, they examined the perception of teachers sharing the same teaching context with each other and with the learner participants. Moreover, the learners in these studies were secondary school L2 learners. Studies that involved learners at other academic levels such as university students would also be useful. Additionally, these studies investigated teacher perception from the perspectives of lexical difficulty based on the assumption that teachers who are more aware of the difficulties that their students cope with are more likely to focus their teaching on that aspect to address their students' needs (McNeill, 2005). The same argument can be applied for word usefulness. If teachers think the words are useful for their learners to know, they are likely to put more effort to help their learners acquire these words. Therefore, research which determines the relationship between teacher perception of word usefulness and learner vocabulary knowledge is also valuable.

\subsubsection{Teacher perception of vocabulary learning and instruction in general}

Eight studies have investigated teacher cognition of vocabulary learning and instruction in general (Gao \& Ma, 2011; Gerami \& Noordin, 2013; Lau \& Rao, 2013; Macalister, 2012; Nural, 2012; Rahimi, 2014; Zhang, 2008; Zimmerman, 2005). These studies are not directly related to word list validation and the relationship between corpora, 
learners, and teachers. However, their findings may provide useful insight into the underlying factors that influence teacher perception in word list implementation; therefore, these studies are briefly reviewed below.

The methods used in these studies varied from teacher self-reported questionnaires (Gao \& Ma, 2011; Macalister, 2012; Rahimi, 2014; Zimmerman, 2005) to interviews (Gao \& Ma, 2011; Gerami \& Noordin, 2013; Lau \& Rao, 2013; Nural, 2012; Rahimi, 2014; Zhang, 2008), scenario-based interviews (Macalister, 2012; Zimmerman, 2005), classroom observation (Gerami \& Noordin, 2013; Lau \& Rao, 2013; Nural, 2012; Zhang, 2008), and field notes (Gerami \& Noordin, 2013; Lau \& Rao, 2013; Nural, 2012; Zhang, 2008). Also, these studies were conducted in different contexts: Hongkong (Gao \& Ma, 2011; Lau \& Rao, 2013), Iran (Gerami \& Noordin, 2013; Rahimi, 2014), Malaysia (Macalister, 2012), Mainland China (Gao \& Ma, 2011; Zhang, 2008), New Zealand (Macalister, 2012), Turkey (Nural, 2012), and the United States (Zimmerman, 2005). The number of teachers participating in these studies also varied: two teachers (Nural, 2012), four teachers (Gerami \& Noordin, 2013; Rahimi, 2014), seven teachers (Zhang, 2008), eight teachers (Lau \& Rao, 2013), 14 teachers (Zimmerman, 2005), 60 teachers (Macalister, 2012), and 250 teachers (Gao \& Ma, 2011).

Despite variation, the results of these studies were consistent with the literature in the field of teacher cognition. Together the research showed that teacher cognition of vocabulary learning and instruction is subject to many factors. Among these factors, prior learning experience (Gao \& Ma, 2011; Rahimi, 2014; Zhang, 2008), teaching experience (Macalister, 2012; Rahimi, 2014; Zhang, 2008; Zimmerman, 2005), and contextual factors (Gao \& Ma, 2011; Gerami \& Noordin, 2013; Lau \& Rao, 2013; Nural, 2012; Rahimi, 2014) are the most influential. Teacher cognition of vocabulary teaching affects their teaching practice, but it is not always the case due to the influence of contexts such as parents/ society expectation, university entrance exams, curricula, materials, resources, and students' characteristics (Gerami \& Noordin, 2013; Lau \& Rao, 2013; Nural, 2012; Zhang, 2008). Although not directly related to word list studies, these findings are meaningful. They suggest that teacher cognition of vocabulary instruction is shaped by many factors; therefore, examining teacher perception of the items from corpus-based word lists can provide an implicit picture of the actual learning and teaching of these words in L2 classrooms. 
Taken together, this section has shown that teacher cognition of vocabulary is still somewhat lacking in both teacher cognition research and vocabulary research. Among the few studies investigating this issue, only two studies into word list validation involve teachers. Together, these studies suggest that teacher perception of word value is affected by many factors such as prior learning experience, teaching experience, and contextual factors. They also indicate that teacher perception of the value of a word and learner vocabulary knowledge are related. However, the teacher participants in the previous studies shared the same contexts with each other and with the learners. Therefore, it is unclear how the relationship between teacher perception of word value and learner vocabulary knowledge will change according to contexts. Moreover, studies that looked at the relationship between teacher perception and the information from corpora or learners were based on small corpora and a sample of learners lacking diversity in terms of academic levels and language proficiency levels. Additionally, apart from lexical difficulty and instructional value, word usefulness is also an important aspect of teacher cognition. Further research in these areas is warranted.

\subsection{Learner proficiency in word list development}

Learners are the main agents in L2 learning activities (Nation, 2013; Schmitt, 2008). No matter what the corpora and teachers suggest, it is the learners who decide whether to learn the words or not. Examining their vocabulary knowledge may reveal the extent to which the learners have been exposed to the target language (Schmitt, 2010), which helps list developers avoid introducing items that learners already know. This section first focuses on how much we know about L2 learners' vocabulary levels. Then, it examines how learners' proficiency has been considered in the development of previous word lists, especially academic word lists.

\subsubsection{L2 learners' vocabulary knowledge}

Nine studies have investigated the vocabulary levels of learners in a wide range of contexts: China, Denmark, India, Indonesia, Israel, Taiwan, and Vietnam. Three of them were conducted at the secondary school level (Henriksen \& Danelund, 2015; Laufer, 1998; Stæhr, 2008) while six studies focused on the university level (Barnard, 1961; Matthews \& Cheng, 2015; Nguyen \& Webb, 2016; Nurweni \& Read, 1999; Quinn, 1968; Webb \& Chang, 2012). Despite the variation in the contexts and participants, these studies consistently showed that learners know more words at higher frequency levels than lower frequency levels. Experimental research on learners' knowledge of 
multi-word units also indicated that L2 learners are sensitive to frequency. Ellis et al.'s (2008) experiments on advanced L2 learners' knowledge of the formulas from their AFL showed that these learners' language processing is influenced by the frequency of the formulaic expressions. Similar results were reported by Hernández, Costa, and Arnon (2016) who investigated upper intermediate and lower advanced L2 learners' responses to high and low frequency words and phrases. Together previous studies suggest that L2 learners tend to encounter high frequency items more often than low frequency items and are more likely to know them better (Ellis, 2002a, 2002b). Despite this common trend, previous research also shows that learners' vocabulary levels are varied. Let us examine the nine studies which estimated the vocabulary levels of L2 learners in detail. The three studies with secondary school students are reviewed first, and are followed by those with university students.

\subsubsection{Vocabulary levels of secondary school students}

All of the three studies with secondary school students were conducted with a small sample of learners and used West's (1953) GSL to represent general high-frequency words. Yet, their findings provide useful insight into the vocabulary levels of L2 learners.

Laufer (1998) measured the vocabulary knowledge of two groups of Hebrew EFL secondary school students in Israel: 26 grade 10 learners and 22 grade 11 learners. The years of formal English instruction of the two groups were six years and seven years, respectively. The learners' receptive vocabulary sizes were measured by Nation's (1990, 1983) VLT while their productive vocabulary sizes were measured by Laufer and Nation's (1999) Productive Vocabulary Levels Test (PLT). The results showed that, the grade 10 learners only mastered 1,900 words receptively and 1,700 words productively. In contrast, the grade 11 learners knew 3,500 word families receptively and 2,550 words productively. The percentage of the most frequent 2,000 word families known by these learners is presented in Table 2.4.

Table 2.4. Percentage of the most frequent 2,000 words known by Laufer's (1998) learners $(\%)$

\begin{tabular}{lrr}
\hline Vocabulary knowledge & Grade $10(N=26)$ & Grade $11(N=22)$ \\
\hline Receptive & 73.55 & 92.78 \\
Productive & 65.72 & 83.33 \\
\hline
\end{tabular}


Studies with EFL secondary school learners in Denmark, however, reported a much lower vocabulary level. Stæhr (2008) used Schmitt et al.'s (2001) VLT to measure the receptive vocabulary levels of 88 Danish EFL grade 9 students. These students had studied English for seven years. It was found that $77.27 \%$ of the learners had not mastered the most frequent 2,000 words receptively. Henriksen and Danelund's (2015) series of three studies revealed similar results. Their first two studies were conducted with 26 grade 10 learners. Schmitt et al.'s (2001) VLT was used to measure the learners' receptive vocabulary sizes while Laufer and Nation's (1999) PLT was used to measure their productive vocabulary size. The results showed, despite spending seven to eight years studying English, these learners only knew $60.09 \%$ of the most frequent 2,000 words receptively and $54.70 \%$ of these words productively. The analysis of individual learners shows that more than $80 \%$ of the learners had not mastered the most frequent 2,000 word families receptively, and more than $88 \%$ had not mastered these words productively. The third study was conducted with 27 grade 10 and 29 grade 12 learners. The grade 12 learners had an additional 225 hours of instruction compared with the grade 10 learners. Similar to the first two studies, Schmitt et al.'s (2001) VLT and Laufer and Nation's (1999) PLT were used to measure learners' receptive and productive vocabulary knowledge, respectively. However, in this study, the 1,000 word frequency level items from Nation's (1983) receptive VLT were included to measure learners' knowledge of the first 1,000 word families. Results of this study indicated an alarming situation about the vocabulary levels of Danish EFL secondary school students. $11.11 \%$ of the grade 10 learners had not mastered the most frequent 1,000 word families while $33.33 \%$ of the grade 10 learners and $20.69 \%$ of the grade 12 learners had mastered only the most frequent 1,000 word families.

\subsubsection{Vocabulary knowledge of university learners}

Research with EFL university learners revealed a similar picture to those with secondary school students. Barnard (1961) measured the knowledge 1,253 first-year university students in India had of the GSL words. In this study, the students took a translation test in which they were provided with sentences containing the target words and had to translate the meaning of these words into their L1. The result showed that the students knew only around 1,500 words. Quinn (1968) replicated Barnard's (1961) study with 214 EFL first-year university students in Indonesia. The students in his study also participated in a translation test with the GSL words used as the target words. The 
results showed that these learners knew only around 900-1,000 words despite having at least six years of English instruction.

Nurweni and Read's (1999) study with 1,447 learners in the same learning context with Quinn's (1968) learners revealed the same results. Similar to Barnard (1961) and Quinn (1968), a translation test was used to measure learners' knowledge of 143 GSL words. The estimated results of the translation tests showed that, on average, the learners understood the meaning of only $60 \%$ of the $1^{\text {st }} 1,000$-words and $37 \%$ of the $2^{\text {nd }} 1,000$ words. If a score of $90 \%$ correct answers was set as the criterion to indicate acquiring a level, less than $6 \%$ of the learners had mastered the $1^{\text {st }} 1,000$-word level, and none of the learners had mastered the $2^{\text {nd }} 1,000$-word level.

Webb and Chang (2012) conducted a longitudinal study which measured the receptive vocabulary knowledge of 166 EFL learners in Taiwan over five years (including formal English instruction at secondary and university levels). Schmitt et al.'s (2001) VLT and the first 1,000 word frequency level from Nation's (1983) VLT were used as the instrument. They found that, despite over nine years of formal English instruction, only $47 \%$ of the learners had mastered the $1^{\text {st }} 1,000$-word level, and $16 \%$ had mastered the $2^{\text {nd }} 1,000$-word level.

Nguyen and Webb (2016) investigated the receptive vocabulary knowledge of 100 Vietnamese EFL first-year university English-major students. Webb et al.’s (n.d.) New Vocabulary Levels Test was used to measure the learner receptive vocabulary knowledge. This study revealed that, after at least seven years of learning English, the learners had mastered the $1^{\text {st }} 1,000$-word level, but had not mastered the $2^{\text {nd }} 1,000$-word level. In fact, on average, they knew only $77.83 \%$ of the $2^{\text {nd }} 1,000$-words.

Unlike the studies above, Matthews and Cheng (2015) focused on L2 learners' knowledge of the spoken forms of general high-frequency words. Their study was conducted with 167 EFL university students in China, who had studied English for at least 10 years. They measured learners' receptive knowledge of 89 high-frequency words from the $1^{\text {st }}, 2^{\text {nd }}$ and $3^{\text {rd }} 1,000$ word levels from Nation's (2012) BNC/COCA frequency lists. The learners were given written sentences with one target word missing. They then listened to the recording of the sentences once and filled in the blanks with the missing words. The results showed that learners knew only $81.96 \%$ of the $1^{\text {st }} 1,000$ words, $73.98 \%$ of the $2^{\text {nd }} 1,000$-words, and $60.71 \%$ of the $3^{\text {rd }} 1,000$-words. 
It can be seen that, except for Nguyen and Webb (2016) and Matthew and Cheng (2015), studies investigating the vocabulary levels of university students shared the same limitations as those with secondary school students. That is, they used West's (1953) GSL to represent high-frequency words. As mentioned later in Section 2.8.1.1, the GSL may not reflect current vocabulary as well as more recently created general high-frequency word lists (Gardner \& Davies, 2014; Nation \& Webb, 2011; Richards, 1974). Despite this limitation, together the findings of the studies into the vocabulary levels of secondary school students and university students indicate that the vocabulary levels of L2 learners are diverse. While some learners are able to master the most frequent 2,000 words (Laufer, 1998), others are not (Barnard, 1961; Henriksen \& Danelund, 2015; Laufer, 1998; Matthews \& Cheng, 2015; Nguyen \& Webb, 2016; Stæhr, 2008), and some cannot even master the most frequent 1,000 words (Henriksen \& Danelund, 2015; Nurweni \& Read, 1999; Quinn, 1968; Webb \& Chang, 2012). Given that word lists should suit the level of list users (Nation, 2016), let us look at how the variation in learners' proficiency has been considered in the development of existing word lists.

\subsubsection{How are existing word lists adaptable to learners' proficiency?}

As can be seen in Section 2.5 above, there are two different approaches towards identifying academic vocabulary. The first approach is assuming that learners know general high-frequency vocabulary and looking for lower frequency words that have wide range and high frequency in academic texts (Browne et al., n.d.; Campion \& Elley, 1971; Coxhead, 2000; Nesi, 2002; Praninskas, 1972; Simpson-Vlach \& Ellis, 2010; Xue \& Nation, 1984). The second approach (Gardner \& Davies, 2014) does not assume that learners know general high-frequency vocabulary. Instead of relying on any ready-made lists to distinguish general high-frequency words from academic words, all items that have wider range and higher frequency in academic than non-academic texts are included. These approaches are also common ways to select items for discipline-specific word lists. Most of these lists followed the first approach (Coxhead \& Hirsh, 2007; Hsu, 2013, 2014; Khani \& Tazik, 2013; Konstantakis, 2007; Liu \& Han, 2015; Martínez, Beck, \& Panza, 2009; Valipouri \& Nassaji, 2013; Wang, Liang, \& Ge, 2008) while several adopted the second approach (Lei \& Liu, 2016; Ward, 1999, 2009). Both approaches provide useful ways of determining the most frequent, wide ranging, and evenly distributed words in academic and specialized texts for L2 learners to acquire. The first approach enables learners and teachers to avoid repeatedly learning and 
teaching known items while the second approach allows academic word lists or discipline-specific word lists to avoid limitations related to ready-made general highfrequency word lists. However, both approaches share the same limitation; they look at learners as a homogeneous group that has the same starting point of vocabulary learning. As reviewed in the previous section, the vocabulary levels of L2 learners are varied. Given that all previous word lists were created for a homogeneous group, further efforts are needed to make word lists more adaptable to the diversity of learning contexts and vocabulary levels of list users.

\subsubsection{Summary}

Previous studies have shown that learners are sensitive to frequency and are more likely to know high-frequency lexical items than low-frequency items. Also, these studies found that the vocabulary levels of L2 learners in different EFL contexts are varied whereas existing word lists were created for a homogeneous group with the same vocabulary levels. Therefore, to better support learners' continual vocabulary development, further research on academic word lists should consider the variation in learners' proficiency.

\subsection{General high-frequency word lists}

The previous section has suggested that learners' proficiency should be taken into account in the development of academic word lists. Given the common assumption that learners already know general high-frequency words before they start learning items from academic word lists (e.g., Coxhead, 2000; Xue \& Nation, 1984), this section closely examines research on general high-frequency word lists. It begins with a review of existing general high-frequency word lists. Then, it examines previous studies which determined the most suitable general high-frequency word list for L2 learners. After that, it looks at the value of general high-frequency words in academic spoken English.

\subsubsection{What are existing general high-frequency word lists?}

To date, West's (1953) GSL is the best known and most influential list of general highfrequency words. However, this list has been criticized for its age (Carter \& McCarthy, 1988; Nation, 2004; Nation \& Webb, 2011; Richards, 1974; Schmitt, 2010). Several lists of general high-frequency vocabulary have been created recently with the aim to replace the GSL. This section describes the GSL and these lists in turn. 


\subsubsection{West's (1953) General Service List}

West's (1953) GSL was developed from a five-million running word corpus of written texts. Frequency was the most important criterion for selecting words. It was used to ensure that the GSL words are lexical items that L2 learners will often encounter and use. The frequency data information used in the GSL is based on Thorndike and Lorge's (1944) word counts. According to West (1953), although frequency is the most important criterion to select the GSL words, five other criteria were also used in vocabulary selection so that the GSL would consist of words suitable for the teaching of English to foreigners. The other criteria are ease of learning, necessity, cover, stylistic level, and emotional neutrality. In terms of ease of learning, a number of words which have similar forms with those having high frequency were also included in the GSL though their frequency is not high. This decision is based on the assumption that learners will need little effort to learn these items because of their close relationship to the high frequency items. In terms of necessity, low frequency items were still included in the GSL if they could cover a certain range of necessity ideas. In contrast, in terms of cover, the items near the root sense were more likely to be included than those distant from the root sense. For instance, although for the time being and for the present have the same meaning, for the present is preferred because present is used in its root sense while being is rather distant in meaning from to be. In terms of stylistic level and emotional neutrality, a number of stylistically marked expressions were not included in the GSL despite their high frequency because West (1953) assumed that ESL/EFL learners used English to express ideas rather than to express emotion.

When tested in different kinds of texts, the GSL provided coverage from $65.46 \%$ to $91.90 \%$ (Table 2.5). With its impressive coverage, the status of the GSL has been established for a long time, and it has a huge impact on L2 learning and teaching practice and vocabulary research. The GSL has been suggested as the starting point for L2 vocabulary learning (Nation, 2013), and has been widely used as the basis for early graded reader schemes (Nation, 2004). This list has been used in the construction of vocabulary tests such as the VLT (Nation, 1983; 1990; Schmitt et al., 2001). It was considered as the general high-frequency vocabulary baseline of a number of specialized word lists such as Praninskas's (1972) American University Word List, Xue and Nation's (1984) UWL, Coxhead's (2000) AWL (see Section 2.5.2 for these lists), Coxhead and Hirsh's (2007) EAP Science Word List, Khani and Tazik's (2013) academic word list for applied linguistics research, Valipouri and Nassaji’s (2013) 
Table 2.5. Coverage of West's (1953) GSL in different text types

\begin{tabular}{|c|c|c|c|}
\hline Studies & Kinds of text & Size (tokens) & Coverage $(\%)$ \\
\hline Hirsh \& Nation (1992) & Novels for L1 teenagers & $26,479-34,909$ & 89.7-91.9 \\
\hline \multirow{2}{*}{ Sutarsyah, Nation \& Kennedy (1994) } & The EAP corpus & 311,768 & 78.43 \\
\hline & The ESP corpus & 295,294 & 82.5 \\
\hline Nation \& Hwang (1995) & Lancaster-Oslo-Bergen Corpus & 1 million & 82.3 \\
\hline Ward (1999) & Engineering texts & 60,000 & 79.8 \\
\hline Coxhead (2000) & Written academic corpus & $3,513,330$ & 76.1 \\
\hline Cobb \& Horst (2004) & $\begin{array}{l}\text { The Learned section of the Brown } \\
\text { corpus }\end{array}$ & 14,283 & 73.32 \\
\hline \multirow{4}{*}{ Nation (2004) } & Academic corpus & 3.5 million & 75.5 \\
\hline & Economic text & 300,000 & 82.5 \\
\hline & London-Lund spoken corpus & 500,000 & 89.6 \\
\hline & Fiction & 3.5 million & 87.1 \\
\hline Thompson (2006) & BASE lecture corpus & No information & 86.5 \\
\hline Coxhead \& Hirsh (2007) & $\begin{array}{l}\text { A pilot science corpus of written } \\
\text { academic English }\end{array}$ & $1,761,380$ & 71.52 \\
\hline Hyland \& Tse (2007) & Professional academic writing & $3,292,600$ & 74 \\
\hline Konstantakis (2007) & Business English course books & 600,000 & 85.72 \\
\hline Gilner \& Morales (2008) & ELT material & $1,157,493$ & 86.73 \\
\hline Martínez, Beck \& Panza (2009) & $\begin{array}{l}\text { Research articles in the agricultural } \\
\text { sciences }\end{array}$ & 826,416 & 67.53 \\
\hline
\end{tabular}




\begin{tabular}{|l|l|l|l|}
\hline Studies & Kinds of text & Size (tokens) & Coverage (\%) \\
\hline Coxhead, Stevens \& Tinkle (2010) & $\begin{array}{l}\text { Corpus of secondary science } \\
\text { textbooks }\end{array}$ & 279,733 & 76.96 \\
\hline \multirow{2}{*}{ Li \& Qian (2010) } & $\begin{array}{l}\text { Hong Kong Financial Services } \\
\text { Corpus }\end{array}$ & $6,279,702$ & 72.63 \\
\hline \multirow{2}{*}{ Matsuoka \& Hirsh (2010) } & $\begin{array}{l}\text { An ELT course book (upper- } \\
\text { intermediate) }\end{array}$ & 44,877 & 90.0 \\
\hline \multirow{2}{*}{ Kwary (2011) } & $\begin{array}{l}\text { Chartered Financial Analyst } \\
\text { textbooks }\end{array}$ & 23,719 & 73.33 \\
\hline \multirow{2}{*}{ Khani \& Tazik (2013) } & $\begin{array}{l}\text { Applied Linguistics Research } \\
\text { Articles }\end{array}$ & $1,553,450$ & 76.04 \\
\hline Valipouri \& Nassaji (2013) & Chemistry research articles & 4 million & 65.46 \\
\hline Dang \&Webb (2014) & Academic spoken English & $1,691,997$ & 85.49 \\
\hline \multirow{2}{*}{ Brezina \& Gablasova (2015) } & Lancaster-Oslo-Bergen corpus & 1 million & 84.1 \\
\cline { 2 - 4 } & BNC & 100 million & 82.0 \\
\cline { 2 - 4 } & BE 06 & 1 million & 80.6 \\
\cline { 2 - 4 } & Ententen12 & 80.1 \\
\hline
\end{tabular}


academic word list for chemistry research articles, and Wang et al.'s (2008) Medical Academic Word List.

However, the GSL has three limitations. First, created from texts from the 1930s, the GSL may not accurately reflect current vocabulary (Carter, 1987; Carter \& McCarthy, 1988; Coxhead, 1998, 2000; Hyland \& Tse, 2007; Richards, 1974). Second, the list may be biased towards written English because it was developed from a written corpus (Carter \& McCarthy, 1988). Third, the frequency and range of words beyond the $1^{\text {st }}$ 1,000 GSL word level were not high enough to be included in a general high-frequency word list (Engels, 1968). Therefore, researchers have suggested that the GSL should be replaced or revised by lists which provide a more accurate picture of current vocabulary (Carter \& McCarthy, 1988; Nation, 2004; Nation \& Webb, 2011; Richards, 1974; Schmitt, 2010).

\subsubsection{Nation's (2006) BNC2000}

One candidate to replace the GSL is Nation's (2006) BNC2000. The BNC2000 words are the most frequent 2,000 word families from Nation's (2006) 14 BNC lists. The BNC2000 word families are at Bauer and Nation's (1993) Level 6. The BNC2000 was derived from the 100-million running word BNC corpus. Ten percent of this corpus was from spoken sources, and 90\% was from written sources. Three criteria were used to select the BNC2000 words: frequency, range and dispersion. In terms of range, the selected words should occur in at least 95 of 100 one million-word sections. In terms of frequency, only words which occurred at least 10,000 times in the whole corpus were selected. In terms of dispersion, the selected words should have Juilland and ChangRodríguez (1964) dispersion of at least 0.8. Apart from the three main criteria, some subjective judgments were also made to minimize the bias caused by the formal, written, and adult nature of the BNC. For example, common spoken words (e.g., goodbye, ok, oh), weekdays, months, numbers, letters, and names of countries were also included in the BNC2000 although they do not have high frequency in the corpus.

The strength of the BNC2000 lies in the fact that it provides better coverage than the GSL. The BNC2000 had higher coverage than the GSL in studies comparing the two lists (Gilner \& Morales, 2008; Nation, 2004) (see Section 2.8.2.1 below for the detailed description of these studies). Research on vocabulary load and opportunities for learning also indicates that the BNC2000 provides relatively high coverage $(81.03 \%$ $96.73 \%$ ) in different corpora (Table 2.6). This is much higher than the coverage 
Table 2.6. Coverage of Nation's (2006) BNC2000 in different text types

\begin{tabular}{|l|l|r|r|}
\hline Studies & Kinds of text & Size (tokens) & Coverage (\%) \\
\hline \multirow{5}{*}{ Nation (2004) } & academic corpus & 3.5 million & 83.9 \\
\cline { 2 - 4 } & economic text & 300,000 & 89.8 \\
\cline { 2 - 4 } & London-Lund spoken corpus & 500,000 & 91.1 \\
\cline { 2 - 4 } & Fiction & 3.5 million & 86.6 \\
\hline \multirow{5}{*}{ Nation (2006) } & A novel & 121,099 & 88.09 \\
\cline { 2 - 4 } & Newspapers & About 440,000 & 83 \\
\cline { 2 - 4 } & A simplified text & 10,578 & 91.2 \\
\cline { 2 - 4 } & A children's movie & 9,984 & 86.44 \\
\cline { 2 - 4 } & Unscripted spoken English & 200,000 & 89 \\
\hline Hsu (2009) & General English textbooks & $6,709-44,199$ & $85.22-93.3$ \\
\hline Webb \& Rodgers (2009a) & Movies & $2,841,887$ & 90.67 \\
\hline Webb \& Rodgers (2009b) & TV programs & 264,384 & 89.53 \\
\hline Coxhead, Stevens \& Tinkle (2010) & Secondary science textbooks & 279,733 & 81.03 \\
\hline \multirow{3}{*}{ Webb \& Macalister (2013) } & School journals & 285,143 & 89.88 \\
\cline { 2 - 4 } & Graded readers & 285,143 & 96.73 \\
\cline { 2 - 4 } & Wellington Corpus of Written New Zealand English & 285,143 & 89.76 \\
\hline Dang \&Webb (2014) & Academic spoken English & $1,691,997$ & 89.61 \\
\hline \multirow{2}{*}{ Webb \& Paribakht (2015) } & University admission reading tests & 18,252 & 86.02 \\
\cline { 2 - 4 } & University admission listening tests & 24,176 & 89.83 \\
\cline { 2 - 4 } & University admission cloze tests & 3,438 & 90.71 \\
\hline
\end{tabular}


achieved by the GSL (65.46\% - 91.90\%). However, this does not necessarily mean that the BNC 2000 is the most suitable general service list for L2 learners. Although attempts have been made to include in the BNC2000 words that are common in general spoken English, this list was developed solely from the BNC corpus and is inevitably affected by the British, adult, formal, written nature of this corpus and may not be suitable for designing language courses or materials for L2 beginner learners (Nation, 2004, 2012).

\subsubsection{Nation's (2012) BNC/COCA2000}

A second list which might serve as a more current general high-frequency word list is Nation's (2012) BNC/COCA2000. Similar to the BNC2000, the unit of counting of the BNC/COCA2000 is the Level 6 word family. To address the limitations of the BNC2000, Nation (2012) developed the BNC/COCA2000 from a 10-million running word corpus. $60 \%$ of the corpus was spoken texts from spoken English, movies, and TV programs whereas $40 \%$ was written texts for young children and fiction. Materials from three different varieties of English (British-English, American-English, and New ZealandEnglish) were included in the corpus. Although frequency and range were important criteria in selecting the BNC/COCA2000 words, Nation (2012) also included words that have lower frequency but may be useful in L2 learning contexts. Because the BNC/COCA2000 is quite new, it has not been explicitly compared with other lists. However, its teachingoriented purpose and its derivation from a corpus with a balance between spoken and written texts from different sources, and different varieties of English suggest that the BNC/COCA2000 may be a useful list for L2 learning purposes.

\subsubsection{Browne's (2014) New General Service List}

A third list is Browne's (2014) NGSL which contains 2,801 flemmas (Level 2.5 word families). These words were selected from a 274-million running word corpus derived from the Cambridge English Corpus. Similar to the BNC2000, the corpus used to develop the NGSL was biased toward written texts, which made up $75.03 \%$ of the corpus. According to Browne (2014), frequency, Carroll's dispersion $\mathrm{D}_{2}$, estimated frequency per million (Um), and Standard Frequency Index (Carroll et al., 1971) were used as the criteria to select the NGSL words. Also, feedback from teachers and learners was sought to perfect the list. Yet, no precise descriptions about the cut-off points of these statistical criteria nor the procedure of getting teachers' and learners' feedback have been provided. 
Browne (2014) reported that his NGSL provided higher coverage $(71.34 \%, 81.75 \%)$ than West's (1953) flemmatized GSL (65.87\%, 76.55\%) and Brezina and Gablasova's (2015) flemmatized New General Service List (New-GSL) $(68.68 \%, 78.30 \%$ ) (for information about the New-GSL see Section 2.8.1.5 below) in two magazine corpora (Scientific American, the Economist). It provided higher coverage (85.35\%) than Brezina and Gablasova's (2015) flemmatized New-GSL (82.76\%) in a classical fiction corpus, but lower coverage than the lemmatized version of West's (1953) GSL (86.17\%). However, this does not mean that the NGSL is a better general high-frequency word list than the other two lists for two reasons. First, the NGSL had more items $(2,801)$ than the flemmatized version of Brezina and Gablasova's (2015) New-GSL $(2,228)$ (see below for the description of the New-GSL). Therefore, it is not surprising that the former list provided higher coverage than the latter list. Second, materials from magazines on very specific subjects (science subjects and economics) may not provide an accurate validation of a general high-frequency word list. These materials may contain a large number of academic and technical words because of their specific topics.

\subsubsection{Brezina and Gablasova's (2015) New General Service List}

A fourth list that was recently developed with an aim to replace the GSL is Brezina and Gablasova's (2015) New-GSL. Lemmas (Level 2 word families) were the unit of counting of this list. The New-GSL contained 2,494 lemmas. It was created from four corpora: the BNC, the EnTenTen12, the Lancaster-Oslo-Bergen Corpus (LOB), and the BE06 Corpus of British English (BE06), which comprise a total size of around 12-billion running words. Unlike the other lists, the New-GSL was created from a purely quantitative approach. Three criteria (frequency, dispersion, and distribution across language corpora) were used to select the New-GSL words.

The New-GSL has two strengths. First, the total size of the corpora used to create the NewGSL is larger than the corpora used to create the other lists. Second, it is divided into two main parts: core vocabulary and current vocabulary, which enables teachers and learners to see the change of general vocabulary over time. However, the New-GSL has two limitations. First, it may be biased towards British, written English. Three out of the four corpora (LOB, BNC, BE06), on which the New-GSL were based, represented BritishEnglish, and three out of the four corpora (LOB, BE06, EnTenTen12) were made up of written discourse. In the only corpus which included spoken English (BNC), spoken 
samples accounted for only $10 \%$. Second, the New-GSL was developed from a purely quantitative approach and may not include words that are not very high in frequency but seem to be useful for L2 learning purposes such as hey, hi, and ok.

In brief, this section has described West's (1953) GSL and four recently created general high-frequency word lists. Given the availability of numerous general high-frequency word lists, one key question is: which list is the most suitable for L2 learners? The following section considers this question in detail.

\subsubsection{What is the best general high-frequency word list for $L 2$ learners?}

This section first reviews research on comparing general high-frequency word lists. Then, it points out the limitations of these studies and proposes that teachers and learners should be involved in word list evaluation.

\subsubsection{Previous research comparing lexical coverage of general high-frequency word lists}

Five studies have explicitly compared the GSL with other general high-frequency word lists (Brezina \& Gablasova, 2015; Browne, 2014; Gilner \& Morales, 2008; Nation, 2004; Nation $\&$ Hwang, 1995). All of these studies used lexical coverage (i.e. the percentage of words from a particular word list covered in a text, Nation \& Waring, 1997), as the criterion to evaluate word lists. Lexical coverage has a close relationship with listening comprehension (Bonk, 2000; Stæhr, 2009; van Zeeland \& Schmitt, 2013) and reading comprehension (Laufer, 1989; Laufer \& Ravenhorst-Kalovski, 2010; Schmitt et al., 2011). For this reason, lexical coverage is usually considered as the primary criterion to evaluate word lists. The higher the coverage a word list provides in a text, the better it is.

Most of these studies reported that the GSL did not provide as much lexical coverage as the other general high-frequency word lists while a few of them found that the GSL provided slightly higher coverage. Nation and Hwang (1995) compared the lexical coverage provided by the GSL with two general high-frequency word lists derived from the LOB corpus and the Brown corpus in the LOB corpus. The results showed that in general the GSL provided the lowest average coverage. In a study comparing the GSL plus AWL with the BNC3000, Nation (2004) found that, the GSL plus AWL always provided lower coverage $(85.5 \%, 91.2 \%, 91.4 \%, 88.5 \%)$ than the BNC3000 $(86.5 \%, 93.2 \%, 92.6 \%$, $89.6 \%$ ) in academic texts, economic text, general spoken English, and non-academic 
written English, respectively. Gilner and Morales (2008) compared the lexical coverage provided by GSL with the BNC2000 in a 1.2-million running word corpus made up of eight sub-corpora: interview scripts, short stories, family movie scripts, news articles, novels, ESP readings (technology), the International Herald Tribune, and the New York Times. They found that the BNC2000 provided slightly better coverage (88.08\%) than the GSL $(86.73 \%)$ in the whole corpus and in most of the sub-corpora except for short stories and novels.

As mentioned in Section 2.8.1.4 above, Browne (2014) compared the flemmatized version of the GSL (3,553 fllemmas) with Brezina and Gablasova’s (2015) New-GSL (2,228 flemmas) and his NGSL (2,801 flemmas) in a classical fiction corpus and two magazine corpora. He found that the GSL provided higher coverage than the other two lists in the fiction corpus but lower coverage in the two magazine corpora. The higher coverage of the GSL in the fiction corpus may be due to its larger size than the other lists and the age of the fiction corpus.

Brezina and Gablasova (2015) examined the coverage of the lemmatized version of the GSL (4,114 lemmas) with their New- GSL (2,494 lemmas). They found that the GSL provided higher coverage $(84.1 \% ; 82 \% ; 80.6 \%)$ than the New-GSL $(81.7 \% ; 80.3 \% ; 80.1 \%)$ in the LOB, BNC, and BE06 corpora, but lower coverage (80.1\%) than the New-GSL $(80.4 \%)$ in the EnTenTen 12 corpus. The lower coverage provided by the New-GSL than the GSL in the EnTenTen 12 corpus may be because the New-GSL had far fewer lemmas (2,494 lemmas) than the GSL (4,114 lemmas).

In sum, previous studies suggest that, often, the GSL did not provide as high lexical coverage as other general high-frequency word lists. In some cases, the GSL provided slightly higher coverage which may be because either it has more items than the other lists or the corpora used for the comparison were more advantageous for the GSL.

\subsubsection{Limitations of previous studies}

Despite their valuable findings, studies that compared lexical coverage of general highfrequency word lists have a number of limitations. First, they compared the GSL with only one or two general high-frequency word lists. No studies explicitly compared a larger number of lists. Second, except for Gilner and Morales (2008) and Browne (2014), all studies used the corpora from which the word lists were developed to evaluate the lists. 
Nation and Hwang (1995) used the LOB corpus, the corpus from which the LOB highfrequency word list was derived. Nation (2004) used Coxhead's (2000) academic corpus, the corpus from which the AWL was developed, to compare the GSL plus AWL and the BNC3000. Brezina and Gablasova (2015) used the LOB, BNC, BE06, and EnTenTen12, the four corpora from which the New-GSL was created. This is problematic. For a valid comparison, the corpora used to examine the lexical coverage provided by the lists must be different from the corpora from which the lists were developed (Coxhead, 2000; Nation \& Webb, 2011). Only Nation (2004) avoided this limitation by using three other corpora apart from the corpus on which the list was based. Third, the number of validating corpora in these studies was very small: one corpus (Gilner \& Morales, 2008; Nation \& Hwang, 1995), three corpora (Browne, 2014; Nation, 2004), and four corpora (Brezina \& Gablasova, 2015). Fourth, most of these corpora were written. Of those including spoken materials in the comparison (Brezina \& Gablasova, 2015; Browne, 2014; Gilner \& Morales, 2008; Nation, 2004), spoken materials accounted for a very small proportion compared with written materials. Therefore, studies that compared the lexical coverage of a larger number of general high-frequency word lists in a wide range of spoken and written corpora are needed.

The biggest limitation of these studies, however, is their use of lexical coverage as the sole criterion to compare word lists. Lexical coverage has two limitations. First, the results of a lexical coverage study deeply depend on the nature of the corpus used in a particular study (Nation, 2001; Schmitt, 2010). According to Schmitt (2010), every corpus has limitations. No corpora can completely represent the language that everyone experiences due to the variation in individual environment and interests. Moreover, the constraints of time and accessibility usually make it impossible for the corpus developers to achieve the exact amount and types of language that they would like to include in their corpora. As a result, to some degree, corpora are often biased towards the materials that are easy to collect.

Second, lexical coverage from corpora reflects natural language occurrence. Hence, although lexical coverage can provide L2 learners with the information about the words that they will be likely to encounter in the world (Milton, 2007, 2009), not all of these words are relevant to L2 learning and teaching in a specific context (Daller, Milton, \& Treffers-Daller, 2007; Milton, 2007, 2009). In other words, relying solely on lexical coverage to evaluate corpus-based word lists may not provide a thorough picture of the 
vocabulary that is necessary for L2 learners, which may discourage learners and teachers from using these lists (Simpson-Vlach \& Ellis, 2010; Sorell, 2013).

As discussed in Sections 2.6 and 2.7 above, teachers and learners can provide useful information to help corpus linguists predict outcomes when introducing corpus-based word lists into classrooms. Despite this importance, none of the five studies that compared general high-frequency word lists used information from learners and teachers as criteria. This is a limitation of most corpus-based word lists. To the best of my knowledge, Ellis et al. (2008) is the only study that involved both learners and teachers in the evaluation of a corpus-based word list. This study brings fresh insight into word list validation. However, as pointed out in Section 2.6.1 above, the focus of their study was academic formulas rather than general high-frequency words. Also, their study has several limitations. They did not examine the correlation between the learners' and teachers' data. Moreover, the size of their corpus and the number of teachers and learners in their study were small. Additionally, the teachers and learners in their study are fairly homogeneous.

Taken together, to provide a more thorough assessment of general high-frequency words, future research comparing general high-frequency word lists should make use of information from large and representative corpora, learners at different proficiency levels, and ESL and EFL teachers from a wide range of contexts. Such research will shed light on the most suitable general high-frequency word list for L2 learners as well as the relationship between the information from corpora, teachers, and learners.

\subsubsection{What is the value of general high-frequency words in academic spoken English?}

So far in this section we have reviewed research on the most suitable general highfrequency word lists for L2 learners. Let us now consider the role of general highfrequency words in academic spoken English. Previous studies examining the value of general high-frequency words from different perspectives imply that knowledge of these words is important for L2 learners' comprehension of academic spoken English.

To begin with, Matthews and Cheng (2015) examined the correlation between their learners' scores on their Word Recognition from Speech test with the IELTS listening test. They found that the learners' knowledge of the spoken forms of general high-frequency words was a good predictor of their listening comprehension. This indicates that knowledge 
of general high-frequency words is necessary for listening comprehension in general and comprehension of academic spoken English in particular.

Most studies looked at the value of general high-frequency words from the perspective of lexical coverage. As presented in Section 2.8.1, regardless of the list that represents these words, general high-frequency words always account for the majority of words in a range of discourse types. Compared with mid and low-frequency words, general high-frequency words have a smaller number but offer a larger amount of coverage, which indicates that they have much greater value to L2 learners than words at lower frequency levels.

Importantly, as shown in Table 2.5 and Table 2.6 above, although the coverage may vary according to the text types, the coverage of general high-frequency words in academic spoken English is often higher than in academic written English. West's (1953) GSL covered 85.49\%-86.50\% of academic speech (Dang \& Webb, 2014; Thompson, 2006), but only $65.46 \%-85.72 \%$ of academic writing (e.g., Coxhead, 2000; Konstantakis, 2007; Valipouri \& Nassaji, 2013). Similarly, Nation's (2006) BNC2000 provided higher coverage in academic speech (89.61\%) (Dang \& Webb, 2014) than in academic writing $(81.03 \%$ 89.80\%) (Coxhead, Stevens, \& Tinkle, 2010; Nation, 2004). These findings suggest that perhaps general high-frequency words are more important in academic spoken English than in academic writing. However, it should be noted that previous studies used either West's (1953) GSL or Nation's (2006) BNC2000 to represent general high-frequency words. As pointed out in Section 2.8.2, it is unclear which general high-frequency word list is the most suitable for L2 learners. Moreover, the academic spoken corpora used in the previous research (Dang \& Webb, 2014; Thompson, 2006) were small (fewer than 1.7-million running words) and not very representative (only including lectures and seminars). Further research that examines the coverage of a more updated general high-frequency word list in a larger and more representative academic spoken corpus will provide better insight into the role of general high-frequency words in academic spoken English.

\subsubsection{Summary}

This section has shown that several general high-frequency word lists have been created. However, it is unclear which list is the most suitable for L2 learners. Although attempts have been made to compare these lists, the number of word lists and corpora used in the comparison is limited. Moreover, the corpora lack diversity and were biased toward written 
discourse. Importantly, all studies that compared general high-frequency word lists used lexical coverage as the only criterion. Yet, the information from teachers and learners can reveal the degree to which L2 learners have been exposed to items from corpus-based general high-frequency word lists and how effective the lists are when implemented in L2 classrooms. The important roles of teachers and learners indicate the need to combine information from corpora, teachers, and learners in the validation of general high-frequency word lists. Unfortunately, no attempts have been made to address this need. Therefore, there is a need for a study which compares a large number of general high-frequency word lists using information from corpora, learners, and teachers with great degree of diversity. Such research will shed light on the most useful general high-frequency word lists for L2 learners as well as the relationships between the information from the three sources.

With respect to the role of general high-frequency words in academic spoken English, previous studies examined the coverage of West's (1953) GSL and Nation's (2006) BNC2000 in academic spoken corpora and indicated that these words may be more important in academic speech than in academic writing. However, it is not clear whether the GSL, BNC2000, or another list is the most suitable general high-frequency word list for L2 learners from the perspectives of corpus linguistics, teachers, and learners. Additionally, the academic spoken corpora used in these studies were small. Consequently, the research does not provide clear answers about the relative value of general high-frequency words in academic spoken and written English.

\subsection{Summary of the chapter and rationale for the next chapter}

This chapter has provided the key concepts related to the unit of counting, vocabulary knowledge, and kinds of vocabulary. It shows that, despite the variation in the terminology, all units of counting in word list studies can be placed in Bauer and Nation's (1993) scale that represents seven levels of word families. The unit of counting for a particular word list should suit the characteristics of the list users and the study purposes. In terms of vocabulary knowledge, the chapter shows that knowing a word involves many aspects. Among these aspects, perhaps the relationship between form and meaning is the most important. With respect to the kinds of vocabulary, EAP learners need support from EFL/ESL and EAP teachers to learn general high-frequency and academic words. 
Under the umbrella of academic spoken vocabulary, the chapter first reviewed research on vocabulary in academic spoken English in relation to academic written English. Then, it looked at the roles of teacher perception and learner proficiency in word list validation. Finally, it discussed the role of general high-frequency vocabulary in academic speech. Based on the review, five major gaps in the literature have been identified.

First, previous research has suggested that knowledge of general high-frequency words is important for L2 learners to comprehend academic spoken English. Several general highfrequency word lists have been developed, and several studies have been conducted to determine which list is best. However, these studies compared a limited number of word lists in a small number of corpora. Moreover, the corpora lack diversity and were biased towards written language. The biggest limitation of these studies, however, is their use of lexical coverage as the only criterion. Lexical coverage is an important criterion to evaluate the relative value of word lists because of its close relationship with comprehension. However, given the importance of teachers and learners in L2 vocabulary learning and teaching, lexical coverage should be complemented with the information from teachers and learners in the validation of general high-frequency word lists. Such robust assessment would shed light on the most suitable general high-frequency word list for L2 learners as well as the relative value of these words in academic spoken and written English.

Second, the findings of previous studies suggested that there are relationships between the information from corpora, learners and teachers. However, it is not clear how strong the relationships between these factors are. The only study that involved information from all three sources did not explicitly discuss the relationship between the information from teachers and learners. Moreover, it focused on academic formulas rather than general highfrequency words. Also, the corpora used in the evaluation were small and less representative, and the learners and teachers were fairly homogenous. Therefore, there is a need for a study that explicitly examines the relationship between the information from the three sources.

Third, teacher perception of word value is an underexplored area of both teacher cognition research and vocabulary research. The few studies that have investigated this area involved teachers sharing the same contexts with each other and with the learners. Therefore, it is unclear how teacher perception changes according to context. Moreover, these studies 
looked at teacher perception from the perspectives of lexical difficulty and instructional value. Studies examining teacher perception of word value from other aspects such as word usefulness and with teachers from different contexts would provide further insight into this research area.

Fourth, while research on the vocabulary in academic written English has received a great deal of attention from researchers, little has been done to explore the nature of vocabulary in academic spoken English. The reason for this may be the lack of an academic spoken word list. To date, there is only one academic spoken word list of single words, Nesi's (2002) SAWL. However, little is written about this list. Consequently, it is unclear exactly which words are shared between academic speech from multiple academic disciplines and how similar their distribution is across these disciplines. Nor do we know to what extent these words are different with the core vocabulary in academic written English. To better understand the nature of academic spoken vocabulary, it would be useful to develop an academic spoken word list of single word items.

Fifth, existing spoken and written academic word lists were created with the assumption that learners have the same vocabulary levels when they start learning their lists. However, studies with learners from different EFL contexts show that the vocabulary levels of L2 learners are diverse. Therefore, it is beneficial to create an academic spoken word list that is adaptable to the learners' proficiency.

The remaining chapters of the thesis describe three linked studies which aim to address these gaps. In Study 1 (Chapter 3) and Study 2 (Chapter 4), lexical coverage, learner vocabulary knowledge, and teacher perception of word usefulness are used to determine the most suitable general high-frequency word list for L2 learners. These studies also examine the relationship between the three factors, and how teacher perceptions change according to context. Study 3 (Chapter 5) focuses on the vocabulary in academic speech. It discusses the development of an academic spoken word list (ASWL) for L2 learners who plan to pursue their academic study in English-medium universities. To make the list adaptable to learners' proficiency, the ASWL is then graded into levels based on the best general highfrequency word list that is determined from Studies 1 and 2. To explore the nature of academic spoken vocabulary, the coverage of the ASWL and the best general highfrequency word list is examined in different kinds of discourse (academic speech, academic 
writing, and non-academic spoken English). Moreover, items in the ASWL are compared with those in lists of general high-frequency words and academic written words.

The next chapter presents Study 1, which compares the lexical coverage provided by West's (1953) GSL and three recently created general high-frequency word lists, Nation's (2006) BNC2000, Nation's (2012) BNC/COCA2000, and Brezina and Gablasova's (2015) New-GSL. 


\section{Chapter 3 - Study 1: Lexical coverage of general high-frequency word lists}

\subsection{Introduction}

Chapter 2 indicates a need to identify the most suitable general high-frequency word lists for second language (L2) learners from the perspectives of corpus linguistics, learners, and teachers, as well as determining the relationships between the information from these sources. The first two studies of this thesis are conducted to address these gaps. This chapter presents Study 1 that uses lexical coverage as the criterion for the comparison while the next chapter (Chapter 4) focuses on teacher perception of word usefulness and L2 learner vocabulary knowledge. Together, the findings of the two studies suggest the most appropriate general high-frequency word list for L2 learners. The relationship between the three factors will be addressed in the next chapter where the data from corpora, teachers, and learners are examined together.

This chapter has five sections. Section 3.2 presents the research questions of Study 1. Section 3.3 describes the methodology of this study. Section 3.4 presents the results of the analysis. Section 3.5 summarizes the main findings of the study. Section 3.6 ends the chapter by presenting the rationale for the next study.

\subsection{Research questions}

Study 1 was designed to answer the following questions:

1. Which list of headwords provides the highest average coverage in spoken and written discourse: West's (1953) General Service List (GSL), Nation's (2006) most frequent 2,000 BNC word families (BNC2000), Nation's (2012) most frequent 2,000 BNC/COCA word families (BNC/COCA2000), and Brezina and Gablasova's (2015) New General Service List (New-GSL)?

2. Which list has the highest coverage provided by the most frequent $1,000,1,500$ and 1,996 headwords in spoken and written discourse? 


\subsection{Methodology}

This section presents the methodology of the present study. First, it describes the word lists and corpora used in the study. Next, it justifies the way to compare word lists of different units of counting and different lengths. Finally, it reports the procedure of the study.

\subsubsection{The word lists}

Although there are a number of general high-frequency word lists, analyzing every word list is beyond the scope of a single study. Analysis of established lists that were developed from large corpora using precise and valid methodologies may provide a reliable list that will serve as the vocabulary foundation for L2 learners. Therefore, the present study only focuses on four general high-frequency word lists: West's (1953) GSL, Nation's (2006) BNC2000, Nation's (2012) BNC/COCA2000, and Brezina and Gablasova's (2015) NewGSL (see Section 2.8.1 above for more detailed descriptions of these lists). West's (1953) GSL was chosen because it is the oldest and most influential general high-frequency word list. The other three lists were chosen because they have been created recently. Earlier studies have shown that the BNC2000 and the New-GSL provided higher lexical coverage than the GSL (Brezina \& Gablasova, 2015; Gilner \& Morales, 2008; Nation, 2004). The $\mathrm{BNC} / \mathrm{COCA} 2000$ was chosen because it is the updated version of the BNC2000 and is expected to provide higher lexical coverage than the GSL.

Although Browne's (2014) New General Service List (NGSL) was also created recently, it was not used for the comparison in this study for two reasons. First, no precise description has been provided about the cut-off points of the statistical criteria (frequency and dispersion) that were used to select the NGSL words. Second, preliminary analysis with nine spoken and nine written corpora in this study (see detailed description of these corpora in Section 3.3.2 below) shows that the average coverage per items (multiplied by 1,000) by Browne's (2014) NGSL headwords ranged from $19.68 \%$ to $25.76 \%$. These coverage figures were much lower than those provided by any of the four general high-frequency word lists used in the present study (24.11\%-34.55\%). For these reasons, four word lists were used in this study: West's (1953) GSL headwords, Nation's (2006) BNC2000 headwords, Nation's (2012) BNC/COCA2000 headwords, and Brezina and Gablasova's (2015) New-GSL headwords. The GSL, BNC2000, and BNC/COCA2000 were downloaded as part of Nation, Heatley, and Coxhead's (2002) RANGE package from Paul 
Nation's website (http://www.victoria.ac.nz/lals/about/staff/paul-nation). The New-GSL was downloaded from the online Supplementary Data of Applied Linguistics Journal (http://applij.oxfordjournals.org/content/early/2013/08/25/applin.amt018/suppl/DC1). After being checked for consistency, the number of headwords in each list was 2,168 (GSL), 1,996 (BNC2000), 2,000 (BNC/COCA2000), and 2,228 (New-GSL). The headwords in the GSL, the BNC2000, and the BNC/COCA2000 are Bauer and Nation's (1993) Level 6 word family headwords while those in the New-GSL are Level 2.5 word family headwords (flemmas). In the original version, the New-GSL is a lemma list (Level 2 word family). However, in the present study, the New-GSL lemmas were converted into flemmas (Level 2.5 word families) by removing 266 repeated forms. There are two reasons for this decision. First, distinguishing part of speech has a negative impact on the distinction of very closely related items like smile (v) and smile (n), but cannot distinguish homonyms having the same part of speech like bank (for money) and bank (for river) (Nation, 2016). Second, the use of flemmas also makes it possible to compare the NewGSL with the other lists using Nation et al.'s (2002) RANGE program.

\subsubsection{The corpora}

Eighteen corpora were used in this study (see Table 3.1 and Table 3.2 below). These corpora were in the form of untagged text files. The number of tokens in each corpus ranged from 320,496 to $10,484,320$ in the spoken corpora and from 1,011,760 to $87,602,389$ in the written corpora. These corpora represented a wide range of spoken and written discourse and 10 different varieties of English (American-English, British-English, Canadian-English, Hong Kong-English, Indian-English, Irish-English, Jamaican-English, New Zealand-English, Pilipino-English, and Singapore-English). Moreover, there is a good balance between the number of spoken and written corpora. The purpose of general highfrequency word lists is to provide L2 learners with a solid vocabulary foundation so that they can effectively communicate in English in diverse spoken and written contexts where English is used as a first language, L2, or a lingua franca (Jenkins, 2009; Nation, 2012, 2013). Given the large number of corpora and their great degree of variety, it is expected that this study would provide a more accurate assessment of the lexical coverage provided by each word list. 
Table 3.1. Nine spoken corpora in Study 1

\begin{tabular}{|c|c|c|c|}
\hline Name & Abbreviation & Tokens & Variety of English \\
\hline $\begin{array}{l}\text { British National Corpus } \\
\text { (spoken component) }\end{array}$ & BNC (spoken) & $10,484,320$ & British \\
\hline $\begin{array}{l}\text { International Corpus of English } \\
\text { (spoken component) }\end{array}$ & ICE (spoken) & $5,641,642$ & $\begin{array}{l}\text { Indian, Filipino, Singapore, } \\
\text { Canadian, Hong Kong, Irish, } \\
\text { Jamaican \& New Zealand }\end{array}$ \\
\hline $\begin{array}{l}\text { Open American National Corpus } \\
\text { (spoken component) }\end{array}$ & OANC (spoken) & $3,243,449$ & American \\
\hline Movie corpus (Webb \& Rodgers, 2009a) & Movies & $2,841,573$ & British \& American \\
\hline Wellington Corpus of Spoken New Zealand-English & WSC & $1,112,905$ & New Zealand \\
\hline Hong Kong Corpus of Spoken English & HKCSE & 977,923 & Hong Kong \\
\hline TV program corpora (Rodgers \& Webb, 2011) & TV programs & 943,110 & British \& American \\
\hline London-Lund corpus & LUND & 512,801 & British \\
\hline Santa Barbara Corpus of Spoken American-English & SBCSAE & 320,496 & American \\
\hline Total & & $26,078,219$ & \\
\hline
\end{tabular}


Table 3.2. Nine written corpora in Study 1

\begin{tabular}{llrl}
\hline Name & Abbreviation & Tokens & Variety of English \\
\hline British National Corpus (written component) & BNC (written) & $87,602,389$ & British \\
\hline Open American National Corpus (written component) & OANC (written) & $12,839,527$ & American \\
\hline International Corpus of English (written component) & ICE (written) & $3,467,451$ & $\begin{array}{l}\text { Indian, Filipino, Singapore, } \\
\text { Canadian, Hong Kong, Irish, } \\
\text { Jamaican, New Zealand, \& } \\
\text { American }\end{array}$ \\
\hline Freiburg-Brown corpus of American-English & & American \\
\hline Freiburg-LOB Corpus of British-English & FROWN & $1,024,320$ & British \\
\hline Wellington Corpus of Written New Zealand- English & FLOB & $1,021,357$ & Ben \\
\hline Lancaster-Oslo/Bergen corpus & WWC & $1,019,642$ & New Zealand \\
\hline Brown corpus & LOB & $1,018,455$ & British \\
\hline Kolhapur Corpus of Indian-English & Brown & $1,017,502$ & American \\
\hline Total & KOHAPUR & $1,011,760$ & Indian \\
\hline
\end{tabular}


So far in this chapter, the lists and corpora used for the comparison in the present study have been described. The following two sections discuss in turn two issues that need consideration when comparing the four general high-frequency word lists: the unit of counting and the number of items in the lists.

\subsubsection{How to compare word lists of different units of counting}

For a valid result, the same unit of counting should be used when comparing word lists (Nation, 2016). Most earlier studies (e.g., Gilner \& Morales, 2008; Nation, 2004; Nation \& Hwang, 1995) compared lists using Level 6 word families as the unit of counting. Thus, they did not have to deal with a difference between units of counting. However, three out of the four general high-frequency word lists in this study (GSL, BNC2000, BNC/COCA2000) used Level 6 word family as the unit of counting while one list (NewGSL) used Level 2.5. This makes valid comparisons between the four lists a real challenge because different units of counting may influence the results of corpus-based vocabulary studies (Gardner, 2007; Nation, 2016). There are four options to solve this problem.

The first option is to compare lists in their original format. That is, no changes are made in terms of unit of counting. However, this option is not satisfactory because it favors lists using larger units of counting (e.g., Level 6 word family lists) over lists with smaller units of counting (e.g., flemmas or Level 2.5 word family lists). Level 6 word families include closely related derivations whereas Level 2.5 does not. Therefore, Level 6 word family lists have more members than Level 2 word family lists, and provide higher lexical coverage.

The second option is to convert the Level 2.5 word family list (flemma list) into a Level 6 word family list so that Level 6 word families will be the unit of counting in the comparison. However, this way will overestimate the benefit of the Level 2.5 lists in terms of lexical coverage. The principle behind the use of Level 2.5 word families is that, if learners know the word form (e.g., allow), they may recognize its inflections (e.g., allowed, allowing, allows), but may not recognize its closely related derivations (e.g., allowance, allowances, allowable). In this way, coverage provided by these derivations (e.g., allowance, allowances, allowable) is not counted in the coverage provided by items from Level 2.5 word family list. However, converting a Level 2.5 word family list into a Level 6 word family list would mean counting the coverage provided by these derivations, which 
would overestimate the benefit of a Level 2.5 word family list in terms of lexical coverage. Option 2, therefore, is not reasonable.

The third option is to convert Level 6 word family lists into Level 2.5 word family (flemmas) lists so that Level 2.5 word families will be the unit of counting for the comparison (Brezina \& Gablasova, 2015; Browne, 2014). A Level 6 word family is comprised of both frequent and infrequent Level 2.5 word families. For example, the Level 6 word family allow is made up of three Level 2.5 word families: allow, allowance, and allowable. In the Wellington Corpus of Spoken New-Zealand English (WSC), the lemma allow occurred very frequently (freq=165) while allowance (freq=32) and allowable (freq=4) occurred very infrequently. The rationale behind including infrequent Level 2.5 word families in Level 6 word family lists is that if learners know one member of a Level 6 word family, they may recognize other members even if these members do not occur very frequently. If we convert Level 6 word families into Level 2.5 word families, a flemma list which contains only frequent Level 2.5 word families (e.g., allow) will have advantages over a flemmatized Level 6 word family list that includes both frequent (e.g., allow) and infrequent Level 2.5 word families (e.g., allowance, allowable). Hence, Option 3 may not provide a valid comparison.

A final option is to compare headwords from different lists. That is, inflected forms and derived forms are not counted unless they are headwords. Using headwords has four advantages. First, it minimizes the difference between the numbers of items in each list because other members of the word families will not be included in the comparison. For example, in this study, the Level 6 word family GSL had 2,168 headwords with 11,283 members, while the Level 2.5 word family New-GSL had 2,228 headwords with only 3,214 members. If only headwords are used for the comparison, it will be fairer because the number of items in each list will be around 2,000. Second, using headwords still ensures that the nature of the lists does not change significantly because headwords are usually the most frequent members in word families. However, it should be noted that because family members are not included, coverage will always be less than $100 \%$. This might be seen as a disadvantage of this approach. Third, using headwords also reflects the nature of L2 teaching and learning. That is, L2 teachers and learners usually receive lists of headwords without their inflections and derivations, and thus may choose headwords to teach and learn first. Moreover, they may never focus at all on family members. Fourth, evaluating 
headwords also reflects the principles behind the development of each word list. That is, if learners know one word form such as the headword, they may recognize other Level 2.5 word family members of the headword (from the lemma or flemma approach), or other Level 6 word family members of the headword (from the word family approach). There is no perfect way of comparing word lists of different units of counting. However, the advantages of using headwords for comparisons between lists outweigh the disadvantages. Therefore, headword was chosen as the unit of counting to compare the four general highfrequency word lists in the present study.

\subsubsection{How to compare word lists with different numbers of items}

For a valid comparison, the number of items in word lists should be the same. However, the number of items in the four general high-frequency word lists in this study varied, which raises the need to address this problem. Three ways have been used to compare the lexical coverage provided by word lists: overall coverage, average coverage, and the coverage provided by the most frequent items.

Most earlier studies comparing word lists used overall coverage as the criterion for comparison (Brezina \& Gablasova, 2015; Browne, 2014; Gilner \& Morales, 2008). However, this may not provide a valid comparison for two reasons. First, using overall coverage will favor lists with larger units of counting (e.g., Level 6 word family) over lists that use a smaller unit of counting (e.g., Level 2.5 word family). Second, even if the same unit of counting is used in the comparison, using overall coverage will favor longer lists. For example, the 2,168 GSL Level 6 word families provide overall coverage of $88.33 \%$ in the WSC whereas the 1,996 Level 6 word families from the BNC2000 provided overall coverage of $87.62 \%$. Thus, even though the GSL provides greater coverage, we do not know if it is in fact the better list, because the additional 172 items in the list may be the only reason why it provides more coverage than the shorter list.

Two other ways to compare word lists are to use average coverage and the coverage provided by the most frequent items. Nation and Hwang (1995) used the average coverage

provided by each 100 word families to compare the GSL with other general high-frequency word lists. Nation (2004) and Gardner and Davies (2014) used coverage provided by the most frequent items (i.e. excluding the lowest frequency items from the lists so that each list had the same number of items) to compare word lists in their studies. Each method has 
its strengths and limitations. Average coverage is able to evaluate word lists as a whole. Yet, it does not provide information about the relative value of one item in comparison with other items in a list. Moreover, it favors shorter lists because the extra items in longer lists are likely to be the least frequent items. In contrast, coverage provided by the most frequent items indicates the relative value of the words in the lists. This is useful because lists may be made of very strong items and relatively weak items in terms of lexical coverage. Hence, looking at the most frequent items provides a picture of how the best (and worst) items in terms of lexical coverage compare between lists. However, looking at the most frequent items favors lists with larger numbers of items because a larger number of infrequent items are excluded. Therefore, it may not provide a valid comparison of the lists as a whole. The strengths and limitations of the two approaches can be illustrated by the performance of the GSL and the BNC2000 headwords in the WSC. When the average coverage provided by each headword is compared, the GSL provides lower average coverage $(0.02979 \%)$ than the BNC2000 (0.03201\%). This indicates that as a whole, the BNC2000 is superior to the GSL in terms of lexical coverage. However, when the coverage provided by the most frequent 1,996 headwords is compared, the GSL provides higher coverage $(64.59 \%)$ than the BNC2000 (63.90\%). This is because the GSL had more infrequent headwords excluded (172) than the BNC2000 (0). This suggests that average coverage and coverage provided by the most frequent items on their own may not provide a thorough evaluation of the lists; however, together they may provide a robust assessment of word lists. Thus, both average coverage and coverage provided by the most frequent items were used to evaluate the relative value of the four general high-frequency word lists in the current study.

\subsubsection{Procedure}

The RANGE program (Nation et al., 2002) was used for the analysis. RANGE enables users to analyze the lexical coverage provided by a certain word list in a text. It can be downloaded from Paul Nation's website.

To calculate the average coverage in the corpora provided by each headword list, the overall coverage in the 18 different corpora provided by each list was determined first. This was done by running the corpora through RANGE with each list in turn serving as the baseword list. For example, to find the overall coverage provided by the 2,168 GSL headwords in the WSC (64.59\%), the corpus was run through RANGE with the GSL headword list being the baseword list. Then, the average coverage was calculated by 
dividing the overall coverage by the number of headwords in each list. For example, the average coverage provided by each GSL headword in the WSC was calculated by dividing the overall coverage provided by all 2,168 GSL headwords in this corpus by the number of headwords in the GSL $(64.59 \% \div 2,168=0.02979 \%)$.

To examine the coverage provided by the most frequent headwords in the lists, three cut-off points were selected: 1,000,1,500 and 1,996. The 1,000 cut-off point was chosen because it has been suggested that the most frequent 1,000 words are quite stable and should be included in a general high-frequency word list (Engels, 1968; Schmitt \& Schmitt, 2014). The 1,996 cut-off point was selected because it was the number of headwords in the shortest list (the BNC2000). The 1,500 cut-off point was chosen for two reasons. First, it is near the midpoint between 1,000 and 1,996 headwords. Second, it is useful to look at how the coverage changes at the 1,500 point as well as the 1,000 and 1,996 cut-off points because, as the list gets longer, the additional coverage provided by the added items drops dramatically (Zipf, 1935, 1949), and the items in the list become less stable (Engels, 1968).

To determine the coverage provided by the most frequent 1,000, 1,500, and 1,996 headwords in the GSL, BNC2000, BNC/COCA2000, and New-GSL, headwords in each list were first ranked according to their frequency in different corpora. Then, the coverage provided by each set of the most frequent 1,000,1,500, 1,996 items from each list in each corpus was calculated by adding the coverage of each headword in the set together. For example, to determine the coverage provided by the most frequent 1,000 GSL headwords in the WSC, all 2,168 GSL headwords were ranked according to their frequency in the WSC. The coverage provided by the most frequent 1,000 GSL headwords in the WSC (63.54\%) was the sum of the coverage of each item in the set of the top 1,000 GSL headwords in the WSC.

\subsection{Results}

This section presents the results of the analysis that address the two research questions. First, it focuses on the comparison of the average coverage provided by the four word lists in 18 corpora. Then, it describes the results related to the coverage provided by the most frequent 1,000,1,500 and 1,996 headwords in each list in these corpora. 
Table 3.3. Average coverage of the GSL, BNC2000, BNC/COCA2000 and New-GSL headwords (\%)

\begin{tabular}{|c|c|c|c|c|c|}
\hline & Size & GSL & BNC2000 & BNC/COCA2000 & New-GSL \\
\hline Corpus & (running words) & 2,168 headwords & 1996 headwords & 2,000 headwords & 2228 headwords \\
\hline \multicolumn{6}{|l|}{ Spoken corpora } \\
\hline BNC (spoken) & $10,484,320$ & 30.71 & 33.20 & 33.70 & 31.59 \\
\hline ICE (spoken) & $5,641,642$ & 29.63 & 32.06 & 32.45 & 30.89 \\
\hline OANC (spoken) & $3,243,449$ & 31.46 & 33.31 & 34.55 & 32.03 \\
\hline Movies & $2,841,573$ & 30.12 & 32.65 & 33.09 & 30.86 \\
\hline WSC & $1,112,905$ & 29.79 & 32.01 & 32.70 & 30.50 \\
\hline HKCSE & 977,923 & 29.39 & 33.25 & 32.32 & 30.77 \\
\hline TV programs & 943,110 & 29.89 & 32.41 & 32.88 & 30.89 \\
\hline LUND & 512,801 & 30.60 & 33.81 & 33.35 & 31.41 \\
\hline SBCSAE & 320,496 & 24.11 & 28.32 & 26.40 & 24.39 \\
\hline \multicolumn{6}{|l|}{ Written corpora } \\
\hline BNC (written) & $87,602,389$ & 27.42 & 30.17 & 30.09 & 29.51 \\
\hline OANC (written) & $12,839,527$ & 24.39 & 27.03 & 26.90 & 26.78 \\
\hline ICE (written) & $3,467,451$ & 26.45 & 29.20 & 29.09 & 28.82 \\
\hline FROWN & $1,024,320$ & 26.62 & 29.09 & 29.22 & 28.69 \\
\hline FLOB & $1,021,357$ & 27.33 & 29.96 & 29.93 & 29.37 \\
\hline WWC & $1,019,642$ & 27.60 & 30.16 & 30.28 & 29.56 \\
\hline LOB & $1,018,455$ & 28.45 & 30.88 & 30.94 & 30.04 \\
\hline Brown & $1,017,502$ & 27.86 & 30.18 & 30.39 & 29.55 \\
\hline KOHAPUR & $1,011,760$ & 27.51 & 29.87 & 29.88 & 29.40 \\
\hline
\end{tabular}




\subsubsection{Average coverage}

Table 3.3 above presents the average coverage provided by each headword in the GSL, the BNC2000, the BNC/COCA2000, and the New-GSL. To clarify the significance of the lexical coverage per headword, the figures were multiplied by 1,000. This provided figures that are more in line with studies of lexical coverage. For example, the average coverage provided by each GSL headword in the WSC was 0.02979\%. However, when reporting the result, this figure was multiplied by 1,000 (29.79\%) to enable readers to see the differences more clearly.

The ranking of the lists in terms of average coverage is quite consistent for both written and spoken discourse. The BNC/COCA2000 provided the highest average coverage in most spoken and written corpora. Although the BNC/COCA2000 and the BNC2000 took turns in being ranked first and second, the former list ranked first in 11 out of 18 corpora. The average coverage provided by each list were $26.40 \%$ to $34.55 \%$ (BNC/COCA2000) and $27.03 \%$ to $33.81 \%$ (BNC2000). Moreover, the BNC/COCA2000 provided higher average coverage than the BNC2000 in five out of nine written corpora, which is surprising. The former list was developed from a corpus that consisted of only $40 \%$ written texts. In contrast, the latter was derived from a corpus that was made up of $90 \%$ written texts. Using average coverage as the criterion for evaluation, the New-GSL always ranked third (from $24.39 \%$ to $32.03 \%$ ) while the GSL always ranked last (from $24.11 \%$ to $31.46 \%$ ).

\subsubsection{Coverage provided by the most frequent headwords}

The New-GSL had the highest coverage provided by the most frequent 1,000, 1,500, and 1,996 headwords in spoken and written discourse. Table 3.4 presents the detailed information about the coverage provided by the most frequent 1,000, 1,500 and 1,996 headwords in the four lists while Table 3.5 provides a summary of the information in Table 3.4. No matter which cut-off point was chosen, the New-GSL consistently provided the highest coverage. It ranked first in 17 corpora and second in the remaining corpus (SBCSAE). The fact the New-GSL provided the highest coverage indicates that, despite not performing well as a whole list, the New-GSL might have a number of very frequent items as well as a number of very infrequent items. This is supported by the fact that 139 headwords unique to the New-GSL were among the most frequent 1,000 headwords in at least $50 \%$ of the corpora (e.g., majority, primary). In contrast, 23 headwords unique to the 
Table 3.4. Coverage by the most frequent GSL, BNC2000, BNC/COCA2000 and New-GSL headwords (\%)

\begin{tabular}{|c|c|c|c|c|c|c|c|c|c|c|c|c|c|}
\hline \multirow[t]{2}{*}{ Corpus } & \multirow[t]{2}{*}{ Tokens } & \multicolumn{4}{|c|}{1,000 headwords } & \multicolumn{4}{|c|}{1,500 headwords } & \multicolumn{4}{|c|}{ 1,996 headwords } \\
\hline & & GSL & $\begin{array}{l}\text { BNC } \\
2000\end{array}$ & $\begin{array}{l}\mathrm{BNCl} \\
\mathrm{COCA} \\
2000\end{array}$ & $\begin{array}{l}\text { New- } \\
\text { GSL }\end{array}$ & GSL & $\begin{array}{l}\text { BNC } \\
2000\end{array}$ & $\begin{array}{l}\mathrm{BNC} / \\
\mathrm{COCA} \\
2000\end{array}$ & $\begin{array}{l}\text { New- } \\
\text { GSL }\end{array}$ & GSL & $\begin{array}{l}\text { BNC } \\
2000\end{array}$ & $\begin{array}{l}\mathrm{BNCl} \\
\mathrm{COCA} \\
2000\end{array}$ & $\begin{array}{l}\text { New- } \\
\text { GSL }\end{array}$ \\
\hline \multicolumn{14}{|l|}{ Spoken corpora } \\
\hline BNC (spoken) & $10,484,320$ & 65.29 & 64.25 & 65.80 & 67.81 & 66.26 & 65.68 & 67.01 & 69.39 & 66.57 & 66.26 & 67.40 & 70.12 \\
\hline ICE (spoken) & $5,641,642$ & 62.77 & 61.94 & 63.18 & 65.62 & 63.87 & 63.47 & 64.50 & 67.59 & 64.21 & 63.98 & 64.89 & 68.57 \\
\hline OANC(spoken) & $3,243,449$ & 67.49 & 65.55 & 68.09 & 69.51 & 68.06 & 66.30 & 68.88 & 70.47 & 68.20 & 66.48 & 69.09 & 70.82 \\
\hline Movies & $2,841,573$ & 63.94 & 63.75 & 64.48 & 67.02 & 64.96 & 64.87 & 65.74 & 68.22 & 65.30 & 65.16 & 66.17 & 68.63 \\
\hline WSC & $1,112,905$ & 63.54 & 62.47 & 64.02 & 65.96 & 64.37 & 63.56 & 65.08 & 67.23 & 64.59 & 63.90 & 65.39 & 67.74 \\
\hline HKCSE & 977,923 & 62.82 & 64.92 & 63.57 & 65.93 & 63.57 & 66.10 & 64.48 & 67.67 & 63.71 & 66.37 & 64.63 & 68.40 \\
\hline TV programs & 943,110 & 63.54 & 63.30 & 64.17 & 66.92 & 64.51 & 64.40 & 65.37 & 68.19 & 64.79 & 64.69 & 65.75 & 68.77 \\
\hline LUND & 512,801 & 65.23 & 66.04 & 65.46 & 67.89 & 66.10 & 67.18 & 66.47 & 69.27 & 66.34 & 67.49 & 66.70 & 69.81 \\
\hline SBCSAE & 320,496 & 51.60 & 55.74 & 51.95 & 53.22 & 52.18 & 56.42 & 52.68 & 54.01 & 52.27 & 56.52 & 52.80 & 54.24 \\
\hline \multicolumn{14}{|l|}{ Written corpora } \\
\hline BNC (written) & $87,602,389$ & 57.05 & 57.09 & 57.61 & 60.61 & 58.76 & 59.42 & 59.55 & 63.63 & 59.39 & 60.21 & 60.17 & 65.33 \\
\hline OANC(written) & $12,839,527$ & 51.17 & 51.70 & 51.98 & 55.26 & 52.44 & 53.46 & 53.40 & 57.97 & 52.84 & 53.96 & 53.79 & 59.35 \\
\hline ICE (written) & $3,467,451$ & 55.19 & 55.50 & 55.90 & 59.16 & 56.76 & 57.62 & 57.65 & 62.15 & 57.30 & 58.29 & 58.18 & 63.79 \\
\hline FROWN & $1,024,320$ & 55.46 & 55.25 & 55.99 & 59.10 & 57.12 & 57.43 & 57.88 & 62.06 & 57.68 & 58.06 & 58.44 & 63.61 \\
\hline FLOB & $1,021,357$ & 56.92 & 56.88 & 57.42 & 60.62 & 58.62 & 59.10 & 59.29 & 63.52 & 59.22 & 59.80 & 59.85 & 65.09 \\
\hline WWC & $1,019,642$ & 57.49 & 57.33 & 58.00 & 61.11 & 59.22 & 59.52 & 59.97 & 64.01 & 59.81 & 60.20 & 60.56 & 65.56 \\
\hline LOB & $1,018,455$ & 59.19 & 58.90 & 59.45 & 62.66 & 60.97 & 61.00 & 61.31 & 65.36 & 61.63 & 61.63 & 61.87 & 66.69 \\
\hline Brown & $1,017,502$ & 57.95 & 57.54 & 58.30 & 61.34 & 59.73 & 59.65 & 60.22 & 64.17 & 60.36 & 60.24 & 60.78 & 65.61 \\
\hline KOHAPUR & $1,011,760$ & 57.36 & 57.20 & 57.68 & 61.25 & 59.02 & 59.09 & 59.31 & 63.91 & 59.60 & 59.62 & 59.76 & 65.22 \\
\hline
\end{tabular}


Table 3.5. Range of the coverage by the most frequent GSL, BNC2000, BNC/COCA2000 and New-GSL headwords (\%)

\begin{tabular}{llll}
\hline Word list & 1,000 headwords & 1,500 headwords & 1,996 headwords \\
\hline New-GSL & $53.22-69.51$ & $54.01-70.47$ & $54.24-70.82$ \\
BNC/COCA & $51.95-68.09$ & $52.68-68.88$ & $52.80-69.09$ \\
BNC2000 & $51.70-66.04$ & $53.46-67.18$ & $53.96-67.49$ \\
GSL & $51.17-67.49$ & $52.18-68.06$ & $52.27-68.20$ \\
\hline
\end{tabular}

New-GSL occurred fewer than 10 times in at least 50\% of the corpora (e.g., celebrity, symptom, participant).

The BNC/COCA2000 usually ranked second. It provided higher coverage than the GSL in all 18 corpora irrespective of the chosen cut-off point. Similarly, the BNC/COCA2000 provided higher coverage than the BNC2000 in six out of nine spoken corpora at all three cut-off points. As the number of the most frequent headwords decreased, the number of written corpora in which the BNC/COCA2000 provided higher coverage than the BNC2000 increased. The number of written corpora in which the most frequent 1,996, 1,500 and 1,000 BNC/COCA2000 headwords provided higher coverage than those from the BNC2000 was six, eight, and nine corpora, respectively.

The GSL and BNC2000 took turns ranking last in most cases. The BNC2000 had lower coverage than the GSL in the spoken corpora. It ranked last in six out of nine spoken corpora at all three cut-off points. In contrast, the GSL had lower coverage than the BNC2000 in written corpora. It provided the lowest coverage in three out of nine corpora, eight out of nine corpora and eight out of nine written corpora when the coverage provided by the most frequent 1,000,1,500 and 1,996 GSL headwords was compared.

If average coverage is used as the criterion to evaluate lists, the BNC/COCA2000 might be the most suitable list for L2 learners. However, if coverage provided by the most frequent headwords is used, then a reduced version of the New-GSL might be the most suitable. In other words, the BNC/COCA2000 may be the most suitable list as a whole, but the NewGSL may consist of a very large number of frequent items. As shown in Table 3.6, the difference in the amount of coverage between the cut-off points is quite small. The most 
frequent 1,000 headwords from each list provided coverage ranging from $51.17 \%$ to 69.51\%. In contrast, the next 500 headwords after the most frequent 1,000 headwords only provided additional coverage of $0.57 \%$ to $3.02 \%$. Similarly, the next 496 headwords beyond the most frequent 1,500 headwords added very little coverage, from $0.09 \%$ to $1.70 \%$. This clearly shows a large difference in the relative value of the most frequent 1,000 headwords and the subsequent 996 headwords.

Table 3.6. Coverage by the GSL, BNC2000, BNC/COCA2000, New-GSL headwords at different frequency levels (\%)

\begin{tabular}{llll}
\hline Word list & $\begin{array}{l}\text { 1st 1,000 } \\
\text { headwords }\end{array}$ & $\begin{array}{l}\text { 1001st-1500th } \\
\text { headwords }\end{array}$ & $\begin{array}{l}\text { 1501th-1996th } \\
\text { headwords }\end{array}$ \\
\hline GSL & $51.17-67.49$ & $0.57-1.78$ & $0.09-0.66$ \\
BNC2000 & $51.70-66.04$ & $0.68-2.33$ & $0.10-0.79$ \\
BNC/COCA2000 & $51.95-68.09$ & $0.73-1.97$ & $0.12-0.62$ \\
New-GSL & $53.22-69.51$ & $0.79-3.02$ & $0.23-1.70$ \\
\hline
\end{tabular}

The results of this study show that, depending on the criterion chosen for assessment, either the BNC/COCA2000 or the New-GSL is the best general high-frequency word list in terms of lexical coverage. Perhaps the most clearly apparent finding was that the GSL provided the lowest average coverage in all 18 corpora. Moreover, when the coverage provided by the most frequent 1,000,1,500 and 1,996 headwords was compared, the GSL either ranked third or last. This is surprising because, as mentioned, using coverage provided by the most frequent headwords as the criterion should favor lists with a larger number of headwords because a greater number of infrequent headwords are excluded. In this study, the GSL had the second largest number of headwords (2,168 headwords). Therefore, it could be expected to provide better performance when the coverage provided by the most frequent headwords was compared. Despite this advantage, the GSL ranked last in six out of 18 corpora and third in 12 out of 18 corpora when the coverage provided by the most frequent 1,000 best headwords was compared. It ranked last in 11 out of 18 corpora and third in the remaining seven corpora at both the 1,500 and 1,996 cut-off points. The low coverage of the GSL in Study 1 is consistent with the results of earlier studies by Nation and Hwang (1995), Nation (2004), and Gilner and Morales (2008), which found that the GSL provided 
lower coverage than other lists. The results of the present study also support the recommendation of earlier research (Carter \& McCarthy, 1988; Coxhead, 1998, 2000; Nation, 2004; Nation \& Webb, 2011; Read, 2000; Richards, 1974; Schmitt, 2010) that the GSL should be replaced by a list that represents current vocabulary. An examination of the items in the four lists indicated that a number of words appearing in only the GSL (e.g., haste, inn, shilling, telegram) are infrequent and should be excluded from a current general high-frequency word list. In contrast, a number of frequent words appearing in the other three lists that refer to common technology (e.g., computer, television, technology) or current issues (e.g., diet, energy, environment) did not appear in the GSL. Moreover, words for everyday conversation (e.g., beer, chocolate, job) that appeared in the other three lists were also absent from the GSL. Taken together, the findings suggest that the GSL should be replaced by a list that reflects current language. It can be either the BNC/COCA2000 or the New-GSL.

\subsection{Summary of main findings}

Study 1 has found that the BNC/COCA provided the highest average coverage in spoken and written discourse. In contrast, the New-GSL had the highest coverage provided by the most frequent 1,000,1,500 and 1,996 headwords in spoken and written discourse. These findings suggest that, from the perspective of corpus linguistics, either the

BNC/COCA2000 or the New-GSL is the most suitable general high-frequency word list for L2 learners. These findings form the starting point for Study 2 (Chapter 4) which further compared these lists from the perspectives of teachers and learners. Also, in Chapter 6, these findings will be integrated with those from other studies to answer the question about the most suitable general high-frequency word list for L2 learners (see Section 6.2.2).

This study also reveals that irrespective of the general higher-frequency word lists, the most frequent 1,000 headwords provided a relatively large proportion of coverage in all 18 corpora while headwords beyond this level provided a relatively small proportion of coverage. Chapter 6 will draw this finding together with the findings from the three other studies to discuss the most suitable size for a general high-frequency word list for L2 learners (see Section 6.2.3). 


\subsection{Rationale for the next study}

The present study has found that Nation's (2012) BNC/COCA2000 and Brezina and Gablasova's (2015) New-GSL provided higher lexical coverage than West's (1953) GSL and Nation's (2006) BNC2000 in a range of spoken and written texts. However, it is unclear whether the BNC/COCA2000 or the New-GSL is the most suitable general highfrequency word list for L2 learners. Moreover, this study used lexical coverage as the only criterion to compare these word lists. As pointed out in Section 2.8.2.2, word lists based on lexical coverage may be biased towards the nature of the corpora and may not always be relevant to L2 learning and teaching. To provide a complete picture about the nature of general high-frequency word lists, the next study (Study 2) further compares the BNC/COCA2000 and the New-GSL by using information from teachers and learners. 


\section{Chapter 4 - Study 2: Teacher perception of word usefulness and learner knowledge of general high-frequency words}

\subsection{Introduction}

The previous chapter has shown that Nation's (2012) most frequent 2,000 BNC/COCA word families (BNC/COCA2000) and Brezina and Gablasova's (2015) New General Service List (New-GSL) provided higher lexical coverage than the other two general highfrequency word lists. Built on this finding, Study 2 (this chapter) examines teacher perception of the usefulness and learner vocabulary knowledge of items from the BNC/COCA2000 and the New-GSL. General high-frequency words have been widely accepted as the starting point of second language (L2) vocabulary learning (Nation, 2013; Schmitt, 2010). Therefore, a general high-frequency word list should consist of a large proportion of words perceived as being useful by teachers and known by L2 learners early in their vocabulary development. The findings of this study then help us to address the question about the most suitable general high-frequency word list for L2 learners.

Moreover, this study examines the relationship between lexical coverage, teacher perception of word usefulness, and learner vocabulary knowledge of items from the two lists. Additionally, it investigates the receptive vocabulary knowledge of Vietnamese English as a Foreign Language (EFL) learners. These findings shed light on the relationship between the information from corpora, teachers, and learners as well as completing the picture of L2 learners' vocabulary levels when they start learning items from an academic spoken word list.

Similar to the previous chapter, this chapter has five main sections. Section 4.2 lists the research questions of Study 2 while Section 4.3 presents its methodology. Section 4.4 describes the results of the analysis. Section 4.5 summarizes the main findings of this study. Section 4.6 concludes the chapter with the rationale for the next study. 


\subsection{Research questions}

Study 2 was guided by the following questions:

1. What is the receptive vocabulary knowledge of Vietnamese EFL learners?

2. Which word list, Nation's (2012) BNC/COCA2000 or Brezina and Gablasova's (2015) New-GSL, accounts for a larger proportion of headwords perceived as being useful for L2 learners by experienced teachers of English?

3. Which word list, the BNC/COCA2000 or the New-GSL, accounts for a larger proportion of headwords known by $100 \%, 90 \%$, and $80 \%$ of the learners?

4. Is there a relationship between

(a) lexical coverage and teacher perception of word usefulness

(b) lexical coverage and learner vocabulary knowledge

(c) learner vocabulary knowledge and teacher perception of usefulness of the items from the BNC/COCA2000 and the New-GSL?

\subsection{Methodology}

This section begins with a description of the teacher and learner participants in this study. It is then followed by description of three kinds of instruments used in the study (surveys, Vocabulary Levels Test, and Yes/ No tests), as well as discussion of the target words, and pseudowords found in these tests. The section ends with the procedure of the study.

\subsubsection{Teacher participants}

Eighty-four experienced teachers of English agreed to participate in the present study. However, six of them withdrew during the study because of their busy working schedules. Consequently, 78 teachers remained. These teachers came from three groups with similar sizes: 25 teachers with English as their first language (L1), 26 teachers with Vietnamese as their L1, and 27 with various L1s. The English L1 teachers had experience teaching L2 learners from a wide range of L1 backgrounds in ESL (English as a Second Language)/EFL contexts (e.g., Arabic, Chinese, Ethiopian, French, Iranian, and Portuguese). Their years of experience teaching L2 learners ranged from two to 40 years $(M=13.12, S D=9.35)$. In contrast, most Vietnamese L1 teachers and various L1 teachers experienced teaching English to learners who shared the same L1 background with them in their home countries. The years of experience of Vietnamese L1 teachers ranged from two to $22(M=6.88, S D$ 
$=5.29)$, while that of various L1 teachers was from two to $20(M=8.63, S D=4.64)$. Further information about the nationalities of the teachers is presented in Table 4.1. All 78 teachers had experience teaching English to L2 learners from beginners to advanced levels. The diversity in these teachers' L1 background, teaching contexts, and experience should result in a thorough picture of the perceptions of teachers of English about word usefulness for L2 learners.

Table 4.1. Nationalities of the teacher participants

\begin{tabular}{|c|c|c|c|c|c|}
\hline English L1 teachers & $N$ & Vietnamese L1 teacher & $N$ & Various L1 teachers & $N$ \\
\hline New-Zealander & 13 & Vietnamese & 26 & Indonesian & 6 \\
\hline American & 4 & & & Malaysian & 6 \\
\hline British & 3 & & & Iranian & 2 \\
\hline Canadian & 3 & & & Japanese & 2 \\
\hline \multirow[t]{9}{*}{ Australian } & 2 & & & Taiwanese & 2 \\
\hline & & & & Thai & 2 \\
\hline & & & & Chinese & 1 \\
\hline & & & & Greek & 1 \\
\hline & & & & Jordanian & 1 \\
\hline & & & & Kenyan & 1 \\
\hline & & & & Laotian & 1 \\
\hline & & & & Sri Lankan & 1 \\
\hline & & & & Venezuelan & 1 \\
\hline
\end{tabular}

\subsubsection{Learner participants}

The learners were 346 Vietnamese EFL undergraduate students from 21 intact classes in six universities in Vietnam. Seventy-one students were excluded from the study because they did not complete all the tests. These 71 students spred across all 21 classses. As a result, 275 students remained. Using learners having the same L1 and learning contexts with one group of teacher participants (Vietnamese L1 teachers) made it possible to examine the change in teacher perception of word usefulness according to the degree of familiarity with learners' characteristics and learning contexts. It also allowed the researcher to obtain a 
sufficient number of learners and easily conduct follow-up participant checking about their answers to increase the reliability of the results. The learners' years of studying English ranged from two to 15 years, with an average of $8.92(S D=2.18)$. Based on the normal age of Vietnamese university undergraduate students, it is estimated that the ages of these students were from 18 to 22 years old. The academic majors of these learners varied. Further information about the learners' university levels and academic majors is shown in Table 4.2.

Table 4.2. Learner information $(N=275)$

\begin{tabular}{|c|c|c|c|}
\hline University levels & $N$ & Academic majors & $N$ \\
\hline $1^{\text {st }}$-year & 141 & Computer Sciences \& Technology & 89 \\
\hline $2^{\text {nd }}$-year & 115 & TESOL & 86 \\
\hline \multirow[t]{4}{*}{$3^{\text {rd }}$-year } & 19 & Natural Sciences & 35 \\
\hline & & Law & 31 \\
\hline & & Economics \& Business & 20 \\
\hline & & Social Sciences \& Humanities & 14 \\
\hline
\end{tabular}

\subsubsection{Surveys}

Ten surveys using a five-point Likert scale were created to investigate teacher perception of the usefulness of the 973 target words from the BNC/COCA2000 and the New-GSL (see Section 4.3.6 below for the description of the target words). Point 1 on the scale was labelled as the least useful, and Point 5 the most useful. The surveys were in the Excel format and were emailed to each individual teacher. The written instructions were in English and put at the top of the surveys (see Figure 4.1 below). Detailed instructions of the way to complete the surveys were provided in the recruitment (see Section 4.3.8.1).

Compared with paper-and-pencil or online formats, using the attached Excel format offered a more efficient and effective means of data collection from the teachers in this study. Unlike the paper-and pencil format, emailing the soft copies of the surveys allows researchers to collect data from a geographically disparate population of teachers while causing minimal intrusion into their busy working lives (Dörnyei \& Taguchi, 2010). Unlike online surveys, emailing Excel files enables researchers to obtain a higher rate of responses with more valid data. If all 973 target words had been included in one online survey, it may have either discouraged teachers from participating in the present study or led to fatigue 
effects when completing the survey. Distributing the target words into 10 short surveys for teachers to do when they had time solved this problem. Additionally, emailing the surveys to each individual teacher helped the current researcher to better manage the progress of each participant.

Figure 4.1. Instructions included in the surveys

In the following section, please give your answer to the following question:

"TO WHAT EXTENT IS THE WORD USEFUL FOR YOUR STUDENTS?

(FOR GENERAL PURPOSES, NOT ACADEMIC PURPOSES)

By typing a number from $\mathbf{1}$ to $\mathbf{5}$ in the degree of usefulness column

( 1 is the LEAST useful and $\mathbf{5}$ is the MOST useful).

For example, if you think that knowing the word "haste" is not useful at all, knowing the word "lorry" is relatively useful, and knowing the word "go" is very useful, your response will be as follows.

\begin{tabular}{|r|l|r|}
\hline Number & Word & Degree of usefulness \\
\hline 1 & haste & 1 \\
\hline 2 & lorry & 4 \\
\hline 3 & go & 5 \\
\hline
\end{tabular}

The Likert rating scale format was chosen because it is a useful instrument to explore participants' opinions about nearly any aspect of language learning (Brown \& Rodgers, 2002). The scale survey format provides deep information about the usefulness of the target words. One limitation of this format is that it takes more time to complete due to the task demand. However, because the teachers had the flexibility to complete the surveys when they had time, it was still practical to conduct the scale surveys. A scale of five points was used because, compared with a seven-or 10-point scale, a five-point Likert scale does not make the participants confused between different points on the scale, which minimizes the number of unreliable responses (Dörnyei\& Taguchi, 2010). Compared with a three-point scale, a five-point scale can explore more in-depth information from the participants. A scale with too few points will not provide researchers with enough necessary information from the participants (Dörnyei\& Taguchi, 2010). Moreover, the choice of a five-point Likert scale was supported by a pilot study with three teachers of English (one English L1 teacher, one Vietnamese L1 teacher, and one Chinese L1 teacher). In the pilot study, these 
teachers were asked how many points in a Likert scale they preferred (three, five, seven, or ten). They all agreed that a five-point scale was the most suitable. Furthermore, the fivepoint Likert scale has been used in a number of studies that explored teacher perception (Banister, 2016; Ellis, Simpson-Vlach, \& Maynard, 2008; Macalister, 2012; SimpsonVlach \& Ellis, 2010; Smith \& Tajino, 2003).

Each survey consisted of nearly 100 items. The decision was supported by the results of a pilot study with three teachers of English. In the pilot study, these teachers completed two survey samples: a 100-item survey and a 200-item survey. After completing these surveys, they all agreed that around 100 items per survey were sufficient. In their opinions, completing a 200-item survey was very time-consuming, and it would cause fatigue effects. Stratified randomization was used so that each test contained an equal proportion of BNC/COCA2000 words and New-GSL words. The website www.random.org was used for randomization. The composition of each survey is presented in Table 4.3.

Table 4.3. Distribution of survey items

\begin{tabular}{llll}
\hline & \multicolumn{3}{c}{ Number of survey items } \\
\cline { 2 - 4 } Survey number & COCA/BNC2000 & New-GSL & Total \\
\hline $1-5$ & 54 & 43 & 97 \\
$6-7$ & 55 & 42 & 97 \\
$8-10$ & 55 & 43 & 98 \\
\hline Total & 545 & 428 & 973 \\
\hline
\end{tabular}

\subsubsection{Vocabulary Levels Test}

Schmitt, Schmitt, and Clapham's (2001) Vocabulary Levels Test (VLT) (Version 2) was conducted to measure the receptive vocabulary levels of the learners in the present study. This test was initially developed by Nation $(1983,1990)$ and then revised by Schmitt et al. (2001). The VLT was in the form of word-definition matching (see Figure 4.2 below). Each level of the test has 10 sections. Each section has six words together with three definitions. Test-takers have to choose three out of the six words to match with the three definitions.

The VLT was chosen because it has been validated and widely used by researchers and teachers to measure vocabulary levels in numerous contexts (e.g., Stæhr, 2008; Webb \& Chang, 2012). Moreover, using the VLT makes it possible to compare the vocabulary levels 
of the Vietnamese learners in this study with those in other EFL/ESL contexts reported in previous research.

Figure 4.2. Format of the VLT (Nation, 1983, 1990; Schmitt, Schmitt, \& Clapham, 2001)

$\begin{array}{llll}1 & \text { Copy } & - & \begin{array}{l}\text { End of highest point } \\ \text { This moves a car }\end{array} \\ 3 & \text { Event } & \text { Motor } & \text { Thing made to be like another } \\ 4 & \text { Pity } & & \\ 5 & \text { Profit } & & \\ 6 & \text { tip } & & \end{array}$

In its original version, the VLT had five levels: the 2,000 (2K), 3,000 (3K), 5,000 (5K), 10,000-word (10K) levels and the Academic Word List (AWL). The $2 \mathrm{~K}$ and $3 \mathrm{~K}$ levels might be considered sufficient high-frequency vocabulary to function effectively in English. The $5 \mathrm{~K}$ level represents mid-frequency vocabulary. The $10 \mathrm{~K}$ level focuses on lowfrequency vocabulary while the AWL level examines test takers' knowledge of academic vocabulary. However, the AWL level was not used in the present study because this study aimed to measure learners' knowledge of general high-frequency vocabulary, not academic vocabulary. Similarly, the $10 \mathrm{~K}$ level was removed because the learners in this study were unlikely to have mastered this level. Previous studies that included the $10 \mathrm{~K}$ level to measure L2 learners' vocabulary knowledge reported very few participants having reached this level: no participants (Stæhr, 2008) and two out of 82 participants (Henriksen \& Danelund, 2015). Additionally, many studies that used Schmitt et al.'s (2001) VLT to estimate the vocabulary levels of learners (e.g., Laufer, 1998; Stæhr, 2008; Webb \& Chang, 2012) did not include the $10 \mathrm{~K}$ level because it may be too demanding for their learner participants. Nguyen and Webb's (2016) study with Vietnamese EFL university Englishmajor students showed that their learners had not mastered the $2 \mathrm{~K}$ and $3 \mathrm{~K}$ levels of Webb, Sasao, and Ballance's (n.d.) New Vocabulary Levels Test indicating that there would likely be little value in measuring knowledge of the $10 \mathrm{~K}$ level.

Unlike the surveys for the teachers, the VLT for the learners was in the paper-and-pencil format because response rates to paper-and-pencil surveys tend to be higher than those of online surveys (Dörnyei \& Taguchi, 2010). Moreover, this study involved testing the vocabulary knowledge of a large number of learners (346). Letting the learners take the paper-and-pencil tests during the break between their English lessons with supervision 
ensured that all learners took the tests seriously, and a sufficient number of participants could be recruited. Using a paper-and-pencil format also increased the validity of the data. Unlike the online format, in which the participants are anonymous, the paper-and-pencil format offered the current researcher the opportunities to meet the participants face-to-face and have follow-up participant checking about the options that they did not answer in the tests. To help the learners clearly understand the way to complete the test, the test instructions were written in their L1 (Vietnamese) and put at the top of the tests (see Figure 4.3).

Figure 4.3. Instructions in the VLT in Study 2

Original version

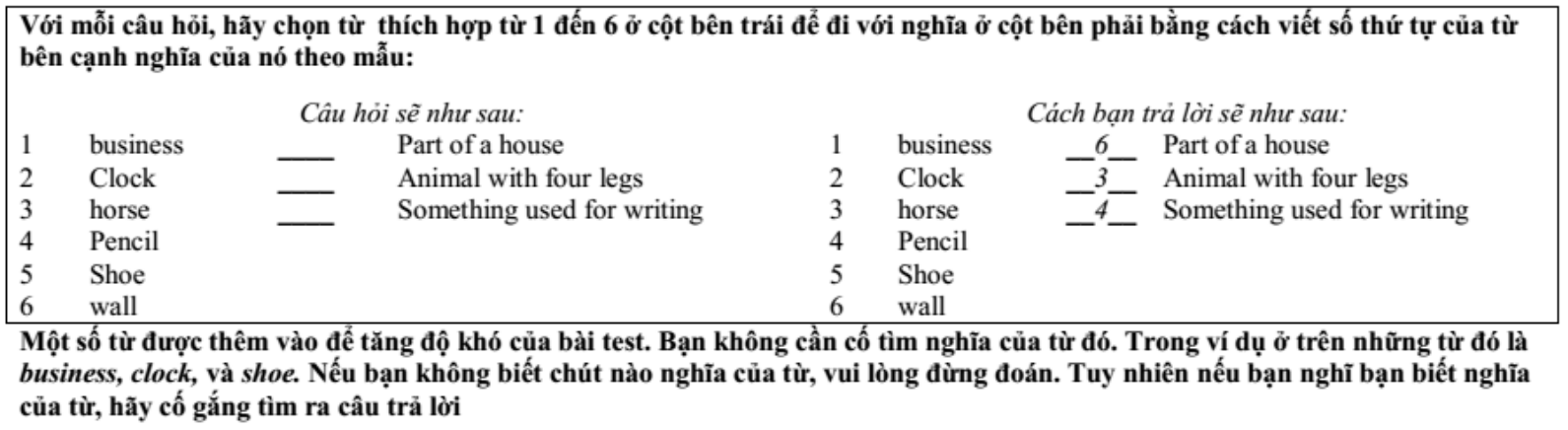

English translation

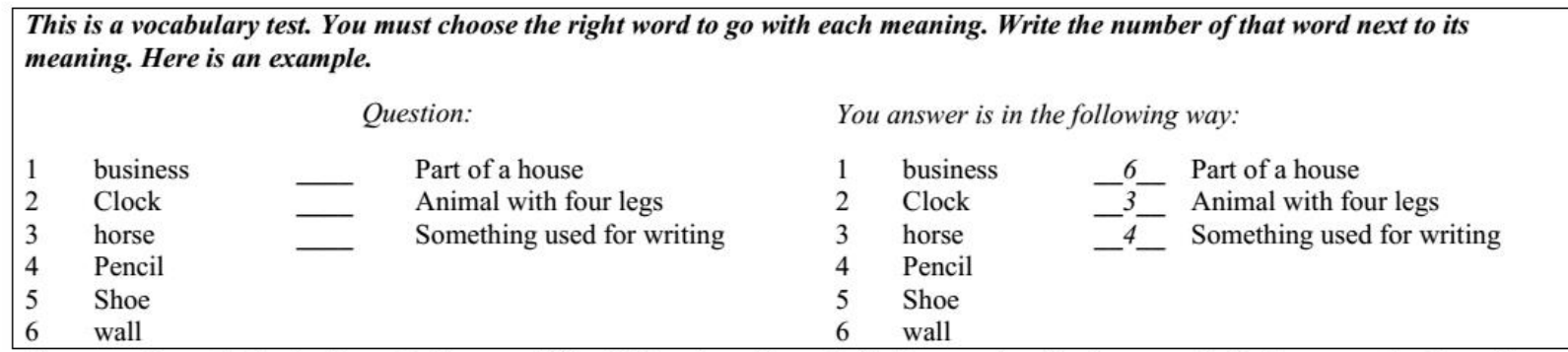

Some words are in the test to make it more difficult. You do not have to find a meaning for these words. In the example above, these words are business, clock and shoe.

If you have no ideas about the meaning of a word, do not guess. But if you think you might know the meaning, then you should try to find the answer

When scoring the VLT in this study, a score of 24 out of 30 was used as the cut-off point for showing mastery of a level. This cut-off point has been used in Xing and Fulcher's (2007) study which examined the reliability of the two VLT versions and in Rodgers' (2013) study of English language learning through viewing television. It is also relevant to the cut-off point (14 out of 18) in Hu and Nation's (2000) study which determined the 
lexical threshold of reading comprehension as they both aim for around $80 \%$ of the correct answers.

\subsubsection{Yes/ No tests}

The previous section described the teacher surveys and learner VLT, this section presents the last instrument from this study, the Yes/No tests. Fifteen Yes/ No tests were designed to measure learners' receptive vocabulary knowledge of the form and meaning relationship of the 973 target words (see Figure 4.4 for the example of the test instruction and Appendix 1 for the test sample). The form and meaning relationship was chosen because it is the most important aspect of knowing a word, and provides the foundation for further development of other aspects (Gardner, 2013; Schmitt, 2008; Webb \& Chang, 2012). The tests were in the paper-and-pencil format. The instructions were in Vietnamese and put at the top of the tests. The reasons for choosing this format and the language of instruction were the same as for the VLT (see Section 4.3.4 above).

Figure 4.4. Instructions in the Yes/No tests

Original version

Với mỗi từ dưới đây, khoanh tròn: "Yes” nếu bạn biết ít nhất 1 nghĩa của từ
"No" nêu bạn không chắc từ đó nghĩa là gì
Lưu ý, một số từ trong bài test không tồn tại trong tiếng Anh. Vì vậy xin bạn hãy thành thật khi đưa ra câu trả lời.

Ví dụ, nếu bạn biết it nhất 1 nghĩa của tì "go", nhung không biết nghĩa của tìn “hapgood", câu trả lời của bạn sẽ nhu sau: $\begin{array}{llllll}\text { 1. go } & \text { 2. hes nopgood } & \text { Yes No }\end{array}$

English translation

For each word, circle "Yes" if you know at least one meaning of the word

"No" if you do not know its meaning

Notes: some words in the test are not real words in English. Please be honest when giving the answers.

For example, if you know at least one meaning of the word "go", but you do not know the meaning of "hapgood", your

$\begin{array}{lll}\begin{array}{l}\text { answer will be as follows: } \\ \text { 1. go }\end{array} \text { (Yes No hapgood } & \text { 2. Yes No }\end{array}$

The Yes/ No test format was chosen because it is the most appropriate format to measure a large number of target words with a large number of participants (Read, 2000; Schmitt et al., 2011). It is a common test format in vocabulary studies (e.g., Anderson \& Freebody, 1983; Meara \& Buxton, 1987; Mochida \& Harrington, 2006). Moreover, this test format is not difficult to construct, administer, or score (Harrington \& Carey, 2009; Meara \& Buxton, 1987; Mochida \& Harrington, 2006). Using the Yes/ No test format also allows a higher 
sampling rate for reliable estimation because a large number of items can be tested in a limited period of time (Beeckmans, Eyckmans, Janssens, Dufranne, \& Van de Velde, 2001; Harrington \& Carey, 2009; Meara \& Buxton, 1987; Mochida \& Harrington, 2006; PellicerSánchez \& Schmitt, 2012; Read, 2000).

One limitation of the Yes/ No test format is face validity. The format does not require test takers to actually demonstrate their vocabulary knowledge, which makes a Yes/ No test not look like a test that measures vocabulary knowledge (Read, 2000). The lack of face validity may lead to the risk of test-takers not taking the test seriously (Nation \& Webb, 2011). Despite this criticism, a large number of studies have found a strong correlation between tests using the Yes/ No test format with those using other formats such as multiple choice (Anderson \& Freebody, 1983; Harrington \& Carey, 2009; Meara, 1996; Meara \& Buxton, 1987; Meara \& Jones, 1988), matching (Mochida \& Harrington, 2006), and cloze (Fairclough, 2011). This suggests that the Yes/ No test format is a valid measure of test takers' receptive vocabulary knowledge (Nation \& Webb, 2011). Moreover, to minimize learners' overestimation of their vocabulary knowledge, the current study followed many earlier studies by including pseudowords as test items in the learner Yes/ No tests (e.g., Meara \& Buxton, 1987; Schmitt, Jiang, \& Grabe, 2011) (see Section 4.3.7 below for detailed explanation of pseudowords).

As shown in Table 4.4, each test has nearly 100 items, which is consistent with previous studies using the Yes/ No test format (e.g., Beeckmans et al., 2001; Cameron, 2002; Harrington \& Carey, 2009; Meara \& Buxton, 1987). Stratified randomization was used to ensure that the proportion of BNC/COCA2000 words, New-GSL words, and pseudowords in each test was the same (see Sections 4.3.6 and 4.3.7 below for more information about these words).

Table 4.4. Distribution of the Yes/ No test items

\begin{tabular}{lllll}
\hline & \multicolumn{4}{c}{ Number of test items in each test } \\
\cline { 2 - 5 } Test number & COCA/BNC2000 & New-GSL & Pseudowords & Total \\
\hline $1-8$ & 36 & 29 & 32 & 97 \\
$9-13$ & 37 & 28 & 32 & 97 \\
$14-15$ & 36 & 28 & 32 & 96 \\
\hline Total & 545 & 428 & 480 & 1,453 \\
\hline
\end{tabular}


So far in this Methodology section, the participants and instruments of the present study have been described, the next two sections focus on the items included in these instruments, including target words and pseudowords.

\subsubsection{Target words}

In this study, 973 target words were used as the test items in the learner Yes/ No tests and the teacher surveys. They were non-overlapping Level 6 word family headwords between the BNC/COCA2000 and the New-GSL (545 BNC/COCA2000 headwords and 428 NewGSL headwords). Headwords were chosen as the unit of counting of the target words for two reasons. First, as mentioned in Section 3.3.3, using headwords reflects the nature of L2 teaching and learning. That is, L2 teachers and learners usually receive lists of headwords without their inflections and derivations, and, therefore, may choose headwords to teach and learn first. Moreover, they may never focus at all on lemmas and family members. Second, headwords are usually the most frequent members of word families, and thus the most likely members to be known.

The headwords were from Bauer and Nation's (1993) Level 6 word family because it is the unit of counting of most earlier academic word lists (e.g. Coxhead's (2000) AWL, Xue and Nation's (1984) University Word List). It is also the primary unit of counting of the Academic Spoken Word List in Study 3 (see Section 5.5 below). Moreover, Nation and Webb (2011) recommend that Level 6 word families are suitable for receptive knowledge. To match this unit of counting, Level 2.5 word family headwords (flemmas) in the NewGSL were converted to Level 6 word family headwords. This was done by grouping flemma headwords belonging to the same Level 6 word family together. For example, two flemma headwords able and ability were listed under the same Level 6 word family headword able. Consequently, the number of New-GSL Level 6 word family headwords was 1,880 .

Only non-overlapping headwords between the two lists were tested. As items appearing in more than one list are likely to be useful and strong items (Nation, 2001; Nation \& Webb, 2011), it may be unnecessary to test the overlapping headwords. Moreover, if all BNC/COCA2000 and New-GSL words were included, it would have resulted in atal number of 2,425 target words. This large number would have made it impractical to conduct the learner Yes/ No tests and teacher surveys in the context of the present study. 


\subsubsection{Pseudowords}

Pseudowords are items that look like real words in the language being tested such as porfume or freath (Meara \& Buxton, 1987). In this study, 480 pseudowords were included in the Yes/No tests to deal with learners' overestimation of their vocabulary knowledge (see Appendix 2). These pseudowords were first taken from Meara's (1992) EFL Vocabulary Tests. Then, they were checked with Nation's (2012) $1^{\text {st }}-25^{\text {th }} 1,000$ BNC/COCA lists, lists of proper nouns, marginal words, compounds, and abbreviations to ensure that these peseudowords did not appear in any of these lists. These pesuedowords were also checked by three native speakers of English to make sure that none of them were real words or proper nouns. After that, they were checked by four Vietnamese L1 speakers with advance level of English proficiency to ensure that the selected pseudowords were not real words or cognates in Vietnamese.

The use of pseudowords is based on the assumption that a person knowing all the words will tick 'Yes' to all the real words, but 'No' to all the pseudowords (Meara \& Buxton, 1987). If the test-takers indicate that they know the pseudowords, their results will be adjusted accordingly (Beeckmans et al., 2001; Read, 2000). This study followed Schmitt et al.'s (2011) approach by excluding the data of participants who checked more than $10 \%$ of pseudowords (see the justification for the 10\% cut-off point in Section 4.4.3.1 below).

Pseudowords were used in this study for three reasons. Firstly, it is a common way to detect learners' overestimation of their vocabulary knowledge in Yes/No test format (Read, 2000). Secondly, several alternatives have been proposed to replace pseudowords such as the Rasch model (Shillaw, 1996) or reaction time (Harrington \& Carey, 2009; Mochida \& Harrington, 2006; Pellicer-Sánchez \& Schmitt, 2012). None of them have proved to be superior to pseudowords. Using the Rasch model makes the Yes/ No test format lose its strength; that is, the format does not need any complex standardization like other test formats (Eyckmans, 2004). Meanwhile, although using reaction time is an interesting idea, its superiority over pseudowords has not yet been confirmed. Pellicer-Sánchez and Schmitt's (2012) study compared the scoring of the Yes/No test adjusted based on the information from the reaction time and from the pseudowords and did not find any significant differences between the two measures. Harrington and Carey (2009) also acknowledged that reaction time alone may not be a sensitive measure of test takers' vocabulary knowledge. Thirdly, from the practicability perspectives, using pseudowords 
seems to be a better option for the present study. It does not require any special facilities that enabled the researcher to administer a series of Yes/ No tests in the paper-and-pencil format with a large number of participants.

The pseudowords accounted for $33 \%$ of the 1,453 test items (480 pseudowords, 545 BNC/COCA2000 words, and 428 New-GSL words). This percentage was chosen because it has been used in a number of earlier studies (Brysbaert, Stevens, Mandera, \& Keuleers, 2016; Meara, 1992; Shillaw, 1996). Nation and Webb (2011) also suggested that pseudowords should comprise one-third of the test items in a Yes/No test. Moreover, 33\% is the most suitable for the present study than other percentages. It is more practical than the 40\% figure used in several earlier studies (Beeckmans et al., 2001; Fairclough, 2011; Harrington \& Carey, 2009; Meara \& Buxton, 1987; Stubbe, 2012). If $40 \%$ had been chosen, 649 pseudowords would have been added to the 973 real words, which would have increased the total number of test items to around 1,622 items. It would then have reduced the number of target words tested in each test, which is impractical (Beeckmans et al., 2001). In contrast, $33 \%$ better guaranteed the validity of the Yes/ No tests in this study than lower percentages: 20\% (Schmitt et al., 2011), 28\% (Harrington \& Carey, 2009), and 29\% (Pellicer-Sánchez \& Schmitt, 2012). The number of target words in these studies was 120 (Schmitt et al, 2011), 72 (Harrington \& Carey, 2009), and 40 (Pellicer-Sánchez \& Schmitt, 2012) whereas in the current study it was 973 . Considering the large number of target words needed to be tested in this study, the $20 \%, 28 \%$, and $29 \%$ figures may be too low and may not guarantee the validity of the Yes/ No tests. For these reasons, 33\% was chosen as the accepted percentage of pseudowords in the present study.

\subsubsection{Procedure}

So far in this Methodology section, the participants, instruments, and test items have been presented. This sub-section describes how the data were collected. It begins with the data collection from the teacher surveys. Then, it presents the procedure of collecting the data from the learner Yes/No tests and the VLT. The data collection was approved by the Human Ethics Committee of Victoria University of Wellington, New Zealand (see Appendices 3 and 4). 


\subsubsection{Teacher survey collection}

The data collection with the teachers was conducted in two stages from December 2014 to May 2015: (1) inviting participants and (2) delivering the surveys. In the first stage, an official invitation was sent to ESL/EFL teachers through different channels such as teacher networks or face-to-face meetings. Teachers who were interested in participating in the study emailed the researcher. The researcher set up one-on-one meetings with the teachers. Most of these meeting were in person while some of them were on Skpye, Facebook, or by emails. In these meeting, the participants were provided with the information sheets (see Appendix 5 for the information sheet) and step-by-step instructions of the way to complete the surveys. They were also given the opportunities to ask for further information about the study. Also, the teachers signed written consent forms (see Appendix 6 for the consent form). Because of their busy working schedules, the teachers were given the flexibility to choose how often and how many surveys would be sent to them each time. To minimize the impact of the variation in the way that teachers responded to the surveys on the results of the study, the teachers were asked to complete the surveys as soon as possible but not to try to finish them all at the same time. They were also asked to have a break when they felt tired. When they were satisfied with their answers, they could send the surveys back to the researcher. In the second stage, the surveys were emailed to each individual teacher in the attached Excel format to match each teacher's working schedule. The teachers downloaded the surveys, completed them, and sent them back to the researcher when they finished. After that, the researcher checked the results, and asked the teachers to provide further information if necessary.

\subsubsection{Learner Yes/No tests and Vocabulary Levels Test collection}

The learner Yes/ No tests and VLT were delivered to 346 learners from 21 intact classes in six universities in Vietnam during the second semester of the Vietnamese university school year from March to April, 2015. The data collection had three main stages: participant invitation, Yes/No tests, and VLT. The tests were conducted during the break between the learners' English lessons. All instructions were given in learners' L1 (Vietnamese) so that the learners were clear about the purpose of the study and the way to complete the tests.

Because of the differences in the participants' English lesson schedules, the schedules for data collection in the 21 classes varied slightly (see Table 4.5). For 19 groups, the learners had six 50-minutes lesson a week while the remaining two groups had only five 50-minute 
English lessons a week. The pilot study showed that it took five to ten minutes to complete one Yes/ No test. Therefore, depending on their English lesson schedules, the learners did three to four tests per week. However, due to other factors such as bad weather, there were instances where the data collection was delayed until the next lesson.

Table 4.5. Schedule for the learner study

\begin{tabular}{|l|l|l|}
\hline \multirow{2}{*}{ Week } & \multicolumn{2}{|c|}{ Learner study schedule } \\
\cline { 2 - 3 } & 19 classes & 2 classes \\
\hline 1 & Participant invitation \\
\hline 2 & Yes/No tests $1,2,3, \& 4$ & Yes/No tests 1, 2, \& 3 \\
\hline 3 & Yes/No tests 5, 6, 7, \& 8 & Yes/No tests 4, 5, \& 6 \\
\hline 4 & Yes/No tests 9, 10,11, \& 12 & Yes/No tests 7, 8, \& 9 \\
\hline 5 & Yes/No tests 13,14, \& 15 & Yes/No tests 10,11, \& 12 \\
\hline 6 & VLT & Yes/No tests 13, 14, \&15 \\
\hline 7 & & VLT \\
\hline
\end{tabular}

The detailed procedure of the data collection was as follows. In the first stage, the researcher met the learners to provide them with detailed explanations of the research and the information sheets (see Appendices 7 and 8), and invited them to participate in the study. Learners who were willing to take part in the study signed written consent forms (see Appendices 9 and 10).

In the second stage, the learners completed $15 \mathrm{Yes} /$ No tests in turn. The purpose of this stage was to determine which BNC/COCA2000 and New-GSL headwords were known to learners. At the beginning of each Yes/ No test, the researcher explained the purpose of the test and the way to complete the test. The learners were informed that the tests were used for research purposes only, and that the test results would not affect their academic results. They were also told that they would be given as much time as they needed to complete the tests. They were asked not to try to guess the words but to only choose "Yes" if they were sure about their meanings. If they knew at least one meaning of the words, they could indicate "Yes", otherwise they should choose "No". The definition of pseudowords and the reasons to use pseudowords in the Yes/No tests were also explained to the learners. The 
researcher also reminded the learners that guessing the meaning from word parts was not encouraged because some pseudowords were made up of parts of real words.

Once the learners had completed all 15 Yes/ No tests, in the third stage, they took the VLT. This stage aimed to examine learners' receptive vocabulary levels. The VLT was conducted after all Yes/ No tests had been completed to avoid possible bias caused by the appearance of 62 target words in the Yes/No tests (e.g., abandon, champion, knit, shy, twelve) in the VLT. The appearance of these items in the VLT is because the $2 \mathrm{~K}$ level measures learners' knowledge of general high-frequency words, and the definitions used in the VLT are general high-frequency words. If the VLT had been conducted before the Yes/ No tests, there could have been the chance that learners did not know the words, but after doing the VLT, they could have checked the words in the dictionary. Later, when doing the Yes/ No tests, the learners might indicate that they knew these words, which might not be true if they did not take the VLT first. Therefore, to avoid bias, the VLT was conducted after all Yes/ No tests had been completed.

Although this order may raise the concern about the bias of the Yes/No test towards the VLT results. This limitation did not seriously influence the result of this study because measuring learners' vocabulary levels was not the primary purpose of this study. Moreover, the learners' vocabulary levels may not have been much improved because of the Yes/ No tests. To retain new words, learners need a considerable amount of time and exposure (Grabe, 2009; Nation, 2013; Webb \& Chang, 2012). The interval between the first Yes/ No test and the VLT was from five to six weeks, and the tested words may not have appeared frequently enough for learning to happen. Moreover, the matching format of the VLT is more demanding than that of the Yes/ No tests, and the VLT also tests learners' knowledge of words from lower frequency levels, not just high-frequency words. Therefore, even if learners may recognize the form of the words due to their experience meeting the words in the Yes/ No tests, they may not be able to give correct answers in the VLT.

When the tests were conducted, the researcher monitored the learners to ensure that they followed the instructions accurately. Data inputting was done immediately after each test was collected. If there were unclear issues related to the learners' answers, the researcher contacted the learners for further checking. 


\subsection{Results}

So far in this chapter, the methodology of the current study has been provided. This section first presents the results of the teacher surveys, learner VLT, and learner Yes/ No tests in turn. Then, it describes the results of the comparison between the data from the teachers and learners in the present study with those from the corpora in Study 1 to determine the relationship between lexical coverage, teacher perception of word usefulness, and learner vocabulary knowledge of the target words.

\subsubsection{Survey results}

To determine teacher perception of the usefulness of the 973 target words, the teacher surveys were examined from two perspectives: (1) the teachers' ratings of the 545

BNC/COCA words and the 428 New-GSL words and (2) the teachers' ratings of the most frequent 420 items from each set of words. The second kind of analysis was included to deal with the difference in the number of items in the two sets of words.

To identify the most frequent 420 items of the 545 BNC/COCA2000 headwords, five steps were followed. First, the frequency of the 545 BNC/COCA headwords in each of the 18 corpora used in Study 1 was determined (see Section 3.3.2 above for more information about these corpora) by running each corpus through Nation, Heatley, and Coxhead's (2002) RANGE with these headwords as the baseword list. Second, the coverage provided by each headword in each corpus was calculated by dividing the frequency of the headword by the number of running words in the corpus, and multiplying by 100 . In the third step, the mean coverage provided by the headword in the 18 corpora was determined by adding the coverage provided by the headword in each corpus together, and then dividing by the number of corpora (18). Mean coverage was used to rank the headwords rather than the combined frequencies because combined frequencies would bias the results towards the findings of the largest corpora. In the fourth step, the 545 headwords were ranked according to their mean coverage in descending order. In the last step, the top 420 headwords were identified. To identify the most frequent 420 items of the 428 New-GSL headwords, the same process was followed.

For each kind of analysis, the data from the three groups of teachers were first analyzed together and then separately. The same four steps were followed to analyze the data from each group. In the first step, the 973 target words were sorted by the mean score given by 
the teachers in the group, and then by the standard deviation $(S D)$. The mean score was sorted in descending order while the $S D$ was sorted in ascending order. In the second step, seven indicators of usefulness were determined:

(1) Mean coverage of four or above

(2) Mean coverage of three or above

(3) The top 100 useful words

(4) The top 200 useful words

(5) The top 300 useful words

(6) The top 400 useful words

(7) The top 500 useful words

In the third step, items that met these criteria were identified and selected for comparison. In the final step, the proportions of the BNC/COCA2000 and the New-GSL words that met each criterion were calculated. A series of $\mathrm{Z}$ tests for two population proportions were conducted to determine whether there existed a significant difference between the proportions of the two lists. The $\mathrm{Z}$ tests were calculated by using the calculator available at http://www.socscistatistics.com/tests/ztest/Default2.aspx.

\subsubsection{Survey results of all teachers}

Table 4.6 presents the results of all 78 teachers. The BNC/COCA2000 always made up a larger proportion of words than the New-GSL irrespective of the criteria of usefulness.

Table 4.6. Proportion of the BNC/COCA2000 and New-GSL rated as useful words by all teachers $(\%)$

\begin{tabular}{|c|c|c|c|c|}
\hline \multirow{2}{*}{ Criterion } & \multicolumn{2}{|c|}{ All 973 target words } & \multicolumn{2}{|c|}{ The most frequent 420 words from each list } \\
\hline & BNC/COCA2000 & New-GSL & BNC/COCA2000 & New-GSL \\
\hline \multicolumn{5}{|l|}{ Mean score } \\
\hline 4 or above & 72.68 & 27.32 & 70.55 & 29.45 \\
\hline 3 or above & 55.96 & 44.04 & 51.38 & 48.62 \\
\hline \multicolumn{5}{|l|}{ Top items } \\
\hline 100 & 79.00 & 21.00 & 77.00 & 23.00 \\
\hline 200 & 71.50 & 28.50 & 68.00 & 32.00 \\
\hline 300 & 66.00 & 34.00 & 60.33 & 39.67 \\
\hline 400 & 61.50 & 38.50 & 57.00 & 43.00 \\
\hline 500 & 60.40 & 39.60 & 54.20 & 45.80 \\
\hline
\end{tabular}


When the teachers' ratings of all 973 target words were examined, the BNC/COCA2000 words comprised from $55.96 \%$ to $79.0 \%$ of the useful words while the New-GSL words accounted for only $21.0 \%$ to $44.04 \%$. Similarly, when the teachers' ratings of the most frequent 420 items from each word list were compared, the BNC/COCA2000 made up from $51.38 \%$ to $77.0 \%$ of the useful words, which is larger than the proportion of the NewGSL (23.0\%-48.62\%). The results of the Z-tests indicate that, except for the case of mean score of 3 or above (the most frequent 420 words), in all cases, the differences were significant at $p<0.05$.

\subsubsection{Survey results of each teacher group}

When the data for each group of teachers were analyzed, the same patterns were found. Irrespective of the criteria of usefulness, the BNC/COCA2000 always provided a significantly larger proportion of useful words than the New-GSL at $p<0.05$. As shown in Table 4.7, the proportion of the BNC/COCA2000 among the most useful words rated by each teacher group was from $55.66 \%$ to $77.52 \%$ (all 973 target words) and from $50.60 \%$ to $77.0 \%$ (the most frequent 420 words). In contrast, the proportion of the New-GSL among the most useful words was only $22.48 \%-44.34 \%$ (all 973 target words) and $23 \%-49.4 \%$ (the most frequent 420 words) (see Table 4.8).

Table 4.9 presents the difference in the proportion of the BNC/COCA2000 and New-GSL words among the most useful words rated by each teacher group. When the data of all 973 target words were compared, the proportion of the BNC/COCA2000 words was $17.44 \%$ $55.04 \%$ (English L1 teachers), 11.32\%-50.0\% (Vietnamese L1 teachers), and 12.38\%$25.0 \%$ (Various L1 teachers) larger than the proportion of the New-GSL among the most useful words. When the most frequent 420 items from each list were compared, the differences in the proportion of the two lists among the useful words was $7.42 \%-54.0 \%$ (English L1 teachers), 1.2\%-48.0\% (Vietnamese L1 teachers), and 2.56\%-26.0\% (Various L1 teachers). As the criterion of usefulness got stricter, the differences between the proportion of the BNC/COCA2000 and the New-GSL become larger. In most cases, the differences were significant at $p<0.05$. 
Table 4.7. Proportion of the BNC/COCA2000 words rated as useful words by each teacher group (\%)

\begin{tabular}{|c|c|c|c|c|c|c|}
\hline \multirow{3}{*}{ Criterion } & \multicolumn{3}{|c|}{ All 973 target words } & \multicolumn{3}{|c|}{ The most frequent 420 words from each list } \\
\hline & $\begin{array}{c}\text { English L1 } \\
\text { teachers }\end{array}$ & $\begin{array}{c}\text { Vietnamese L1 } \\
\text { teachers }\end{array}$ & $\begin{array}{c}\text { Various L1 } \\
\text { teachers }\end{array}$ & $\begin{array}{l}\text { English L1 } \\
\text { teachers }\end{array}$ & $\begin{array}{c}\text { Vietnamese L1 } \\
\text { teachers }\end{array}$ & $\begin{array}{c}\text { Various L1 } \\
\text { teachers }\end{array}$ \\
\hline & $N=25$ & $N=26$ & $N=27$ & $N=25$ & $N=26$ & $N=27$ \\
\hline \multicolumn{7}{|l|}{ Mean score } \\
\hline 4 or above & 77.52 & 66.97 & 62.50 & 76.32 & 64.16 & 59.30 \\
\hline 3 or above & 58.72 & 55.66 & 56.19 & 53.71 & 50.60 & 51.28 \\
\hline \multicolumn{7}{|l|}{ Top items } \\
\hline 100 & 77.00 & 75.00 & 62.00 & 77.00 & 74.00 & 63.00 \\
\hline 200 & 74.50 & 72.50 & 61.50 & 70.00 & 69.00 & 58.00 \\
\hline 300 & 68.33 & 66.00 & 61.67 & 64.00 & 63.33 & 57.00 \\
\hline 400 & 62.50 & 62.72 & 60.75 & 56.75 & 58.00 & 57.00 \\
\hline 500 & 60.00 & 59.80 & 59.00 & 53.80 & 54.60 & 54.60 \\
\hline
\end{tabular}


Table 4.8. Proportion of the New-GSL words rated as useful words by each teacher group (\%)

\begin{tabular}{|c|c|c|c|c|c|c|}
\hline \multirow{3}{*}{ Criterion } & \multicolumn{3}{|c|}{ All 973 target words } & \multicolumn{3}{|c|}{ The most frequent 420 words from each list } \\
\hline & $\begin{array}{c}\text { English L1 } \\
\text { teachers }\end{array}$ & $\begin{array}{c}\text { Vietnamese L1 } \\
\text { teachers }\end{array}$ & $\begin{array}{c}\text { Various L1 } \\
\text { teachers }\end{array}$ & $\begin{array}{c}\text { English L1 } \\
\text { teachers }\end{array}$ & $\begin{array}{c}\text { Vietnamese L1 } \\
\text { teachers }\end{array}$ & $\begin{array}{c}\text { Various L1 } \\
\text { teachers }\end{array}$ \\
\hline & $N=25$ & $N=26$ & $N=27$ & $N=25$ & $N=26$ & $N=27$ \\
\hline \multicolumn{7}{|l|}{ Mean score } \\
\hline 4 or above & 22.48 & 33.03 & 37.50 & 23.68 & 35.84 & 40.70 \\
\hline 3 or above & 41.28 & 44.34 & 43.81 & 46.29 & 49.40 & 48.72 \\
\hline \multicolumn{7}{|l|}{ Top items } \\
\hline 100 & 23.00 & 25.00 & 38.00 & 23.00 & 26.00 & 37.00 \\
\hline 200 & 25.50 & 27.50 & 38.50 & 30.00 & 31.00 & 42.00 \\
\hline 300 & 31.67 & 34.00 & 38.33 & 36.00 & 36.67 & 43.00 \\
\hline 400 & 37.50 & 37.28 & 39.25 & 43.25 & 42.00 & 43.00 \\
\hline 500 & 40.00 & 40.20 & 41.00 & 46.20 & 45.40 & 45.40 \\
\hline
\end{tabular}


Table 4.9. Differences in the proportion of the BNC/COCA2000 and New-GSL words rated as useful words by each teacher group (\%)

\begin{tabular}{|c|c|c|c|c|c|c|}
\hline \multirow{3}{*}{ Criterion } & \multicolumn{3}{|c|}{ All 973 target words } & \multicolumn{3}{|c|}{ The most frequent 420 words from each list } \\
\hline & $\begin{array}{c}\text { English L1 } \\
\text { teachers }\end{array}$ & $\begin{array}{l}\text { Vietnamese } \\
\text { L1 teachers }\end{array}$ & $\begin{array}{c}\text { Various L1 } \\
\text { teachers }\end{array}$ & $\begin{array}{l}\text { English L1 } \\
\text { teachers }\end{array}$ & $\begin{array}{c}\text { Vietnamese L1 } \\
\text { teachers }\end{array}$ & $\begin{array}{c}\text { Various L1 } \\
\text { teachers }\end{array}$ \\
\hline & $N=25$ & $N=26$ & $N=27$ & $N=25$ & $N=26$ & $N=27$ \\
\hline \multicolumn{7}{|l|}{ Mean score } \\
\hline 4 or above & 55.04 & 33.94 & 25.00 & 52.64 & 28.32 & 18.60 \\
\hline 3 or above & 17.44 & 11.32 & 12.38 & 7.42 & 1.20 & 2.56 \\
\hline \multicolumn{7}{|l|}{ Top items } \\
\hline 100 & 54.00 & 50.00 & 24.00 & 54.00 & 48.00 & 26.00 \\
\hline 200 & 49.00 & 45.00 & 23.00 & 40.00 & 38.00 & 16.00 \\
\hline 300 & 36.66 & 32.00 & 23.34 & 28.00 & 26.66 & 14.00 \\
\hline 400 & 25.00 & 25.44 & 21.50 & 13.50 & 16.00 & 14.00 \\
\hline 500 & 20.00 & 19.60 & 18.00 & 7.60 & 9.20 & 9.20 \\
\hline
\end{tabular}


Noticeably, no matter whether the data of the whole group or each group of teachers, or the data of all 973 target words or the most frequent 420 words were analyzed, the BNC/COCA2000 always had a greater proportion of words rated as useful than the NewGSL. So far in this section, the results of the teacher surveys have been presented. Let us now look at the results of the learner VLT and Yes/ No tests in turn.

\subsubsection{Vocabulary Levels Test results}

The data from 71 learners who did not complete all 16 tests (15 Yes/ No tests and VLT) were excluded from the total sample of 346 learners. Consequently, the data from 275 learners were used for the analysis. An SPSS for Microsoft Window Release 23.0 package was used for the statistical analysis. The mean score and SD at each level of the VLT were calculated. A one-way repeated measures ANOVA was conducted to compare learners' scores at the $2 \mathrm{~K}, 3 \mathrm{~K}$, and $5 \mathrm{~K}$ levels. If there were significant differences among the three levels, post-hoc tests were conducted to identify where the difference occurred.

Table 4.10 presents the mean scores of the 275 learners in the VLT. The first row of this table shows that the mean scores of these learners decreased according to the VLT, from 16.42 ( $2 \mathrm{~K}$ level) to 11.01 ( $3 \mathrm{~K}$ level), and then 6.19 (5K level). Results of the one-way repeated measures ANOVA showed that there was statistically significant differences in the mean scores across three levels of the test, Wilks' Lambda $=.22, F(3,272)=326.412, p$ $<.001, \eta^{2}=.78$. Post-hoc tests indicated that knowledge of words at the $2 \mathrm{~K}$ level was significantly higher than at the $3 \mathrm{~K}$ level, which was significantly higher than at the $5 \mathrm{~K}$ level. Normality was in doubt; therefore, the results were supplemented with Friedman's test to confirm the results $(p<0.0005)$. These findings then indicate that the receptive vocabulary knowledge of the learners in this study followed the typical lexical profile. That is, they knew more words at higher frequency levels than words at lower frequency levels. Applying the 24 out of 30 as the cut-off point of mastering a level, it can be inferred from

the first row of Table 4.10 that, as a whole group, the learner participants had not mastered any levels of the VLT. 
Table 4.10. Learners' VLT mean scores $(N=275)$

\begin{tabular}{lllll}
\hline Correct responses & $2 \mathrm{~K}$ & $3 \mathrm{~K}$ & $5 \mathrm{~K}$ & Total \\
\hline Mean & 16.42 & 11.01 & 6.19 & 33.62 \\
Min & 1 & 0 & 0 & 1 \\
Max & 30 & 30 & 30 & 90 \\
$S D$ & 9.66 & 9.38 & 7.47 & 25.17 \\
\hline
\end{tabular}

Table 4.11 provides an overview of the vocabulary levels of the 275 learners. These learners were divided into four groups according to their VLT results: low, mid-low, mid, and high. Learners from the low and mid-low groups had not mastered the $2 \mathrm{~K}$ level. The former group knew less than $50 \%$ of the $2 \mathrm{~K}$ level words while the latter group knew more than $50 \%$. Those from the mid and high group had mastered at least the $2 \mathrm{~K}$ level.

Table 4.11. Vocabulary levels of each learner group $(N=275)$

\begin{tabular}{lll}
\hline $\begin{array}{l}\text { Groups of learners } \\
\text { (vocabulary level) }\end{array}$ & Number of learners & Vocabulary level \\
\hline Low & 140 & $\begin{array}{l}\text { yet to master the 2K level } \\
\text { (scored less than } 15 \text { out of } 30 \text { at the 2K level) }\end{array}$ \\
Mid-low & 37 & $\begin{array}{l}\text { yet to master the 2K level } \\
\text { (scored } 15-23 \text { out of } 30 \text { at the 2K level) }\end{array}$ \\
Mid & 50 & mastered the 2K level \\
High & 48 & mastered the 3K or the 5K levels \\
\hline
\end{tabular}

It can be seen from the table that $64.36 \%$ of the learners (low and mid-low groups) had not mastered the most frequent 2,000 word families. In essence, $50.91 \%$ of the total learners (low group) scored less than $50 \%$ of the correct answers at the $2 \mathrm{~K}$ level. The $2 \mathrm{~K}$ level of the VLT made up of 13 items from the $1^{\text {st }} 1,000$-word level (e.g., ancient, develop, difficult, ) and 17 items (e.g., admire, arrange, clerk, copy, debt) from the $2^{\text {nd }} 1,000$-word level. Because these learners scored less than $50 \%$ of this level, it is unlikely that they had mastered the $1^{\text {st }} 1,000$-word level. 
There were differences between the vocabulary levels of the English-major and the nonEnglish major learners. The English major learners studied TESOL while the non-English major learners studied other subjects (e.g., law, computer sciences). Table 4.12 shows that, on average, the non-English majors had mastered none of the levels of the VLT. In contrast, the right-hand side columns of the table reveal that, the English majors had mastered the $2 \mathrm{~K}$ level $(M=26.94)$, but had not reached mastery of the $3 \mathrm{~K}(M=21.09)$ and $5 \mathrm{~K}$ levels $(M=$ 13.03). This is understandable when considering the Vietnamese EFL context. Englishmajors study English to get an undergraduate or postgraduate degree in English whereas non-English majors study English as part of their programs to obtain an undergraduate or postgraduate degree in other majors (e.g., law, computer sciences, or business) (Hoang, 2010). Therefore, English-majors study English more intensively than non-English majors (Ton \& Pham, 2010). The result of an independent-samples t-test shows that there was a significant difference in the overall scores in the VLT for English majors $(M=61.07, S D$ $=16.74$ ) and non-English majors, $M=21.13, S D=17.19 ; t(273)=18, p<.05$ (two tailed), $\eta^{2}$ $=0.54$. As normality was in doubt, the results were supplemented with a Mann-Whitney U test to confirm the results $(p<0.0005)$. Similar results were found when the scores at the $2 \mathrm{~K}, 3 \mathrm{~K}$ and $5 \mathrm{~K}$ levels were examined. These results suggested that major has an influence on the learners' vocabulary levels.

Table 4.13 shows that neither the $1^{\text {st }}$ year nor $2^{\text {nd }}$ year students mastered any levels of the VLT. The $3^{\text {rd }}$ year students; however, had mastered the $2 \mathrm{~K}$ and $3 \mathrm{~K}$ levels, but not the $5 \mathrm{~K}$. There are two possible reasons for this. First, all $3^{\text {rd }}$ year students were English-majors. Second, their length of studies of English was longer $(M=10.47 ; S D=2.39)$ than the $1^{\text {st }}$ year students $(M=8.37 ; S D=1.91)$, and $2^{\text {nd }}$ year $(M=9.35 ; S D=2.25)$, and previous research has shown that age has influence on vocabulary knowledge (Brysbaert et al., 2016; Nation \& Coxhead, 2014). Despite this fact, the $3^{\text {rd }}$ year students had mastered neither the $3 \mathrm{~K}$ nor the $5 \mathrm{~K}$ levels. 
Table 4.12. Learners' VLT mean score according to academic majors

\begin{tabular}{|c|c|c|c|c|c|c|c|c|}
\hline \multirow{2}{*}{$\begin{array}{l}\text { Correct } \\
\text { responses }\end{array}$} & \multicolumn{4}{|c|}{ Non- English majors $(N=189)$} & \multicolumn{4}{|c|}{ English majors $(N=86)$} \\
\hline & $2 \mathrm{~K}$ & $3 \mathrm{~K}$ & $5 \mathrm{~K}$ & Total & $2 \mathrm{~K}$ & $3 \mathrm{~K}$ & $5 \mathrm{~K}$ & Total \\
\hline Mean & 11.63 & 6.42 & 3.07 & 21.13 & 26.94 & 21.09 & 13.03 & 61.07 \\
\hline Min & 1 & 0 & 0 & 1 & 17 & 2 & 0 & 20 \\
\hline Max & 30 & 30 & 30 & 90 & 30 & 30 & 30 & 89 \\
\hline$S D$ & 7.47 & 6.20 & 4.79 & 17.19 & 3.80 & 7.01 & 7.76 & 16.74 \\
\hline
\end{tabular}

Table 4.13. Learners' VLT mean score according to university year

\begin{tabular}{|c|c|c|c|c|c|c|c|c|c|c|c|c|}
\hline $\begin{array}{l}\text { Correct } \\
\text { responses }\end{array}$ & \multicolumn{4}{|c|}{1 st year $(N=141)$} & \multicolumn{4}{|c|}{ 2nd year $(N=115)$} & \multicolumn{4}{|c|}{ 3rd year $(N=19)$} \\
\hline Min & 1 & 0 & 0 & 1 & 1 & 0 & 0 & 4 & 19 & 8 & 1 & 32 \\
\hline Max & 30 & 29 & 30 & 88 & 30 & 30 & 30 & 90 & 30 & 30 & 25 & 82 \\
\hline
\end{tabular}




\subsubsection{Yes/ No test results}

\subsubsection{Selection of relevant data}

Only data of 112 learners who scored at least 50\% (15 out of 30) of the correct answers at the $2 \mathrm{~K}$ level of the VLT and checked no more than $10 \%$ of the pseudowords in the Yes/ No tests were used for the analysis. They were 32 mid-low level learners, 40 midlevel learners, and 40 high-level learners (for the definition of each group, see Table 4.11 above).

Because 2,000 is commonly accepted as the number of items in a general highfrequency word list (Nation, 2013), data of the mid and high level learners, who had mastered the $2 \mathrm{~K}$ level, was the most relevant. However, given that 2,000 is an arbitrary cut-off of general high-frequency words, data of the mid-low learners whose vocabulary levels were around 2,000 words were also included. Data of low-level learners, however, were not included in the analysis because these learners scored less than 50\% of the correct answers at the $2 \mathrm{~K}$ level, which suggested that they were unlikely to have mastered the $1^{\text {st }} 1,000$ word level.

Only the data from learners checking no more than $10 \%$ of the pseudowords were kept. $10 \%$ was chosen as the acceptable percentage of checked pseudowords for three reasons. First, it is consistent with Schmitt et al.'s (2011) decision. In their study, Schmitt et al. (2011) wanted to know which words from each of the two different texts were known by their participants. Therefore, they made a Yes/No test which consisted of 120 vocabulary items from the two texts, and 30 pseudowords. After that, they excluded data of any participants checking more than $10 \%$ of pseudowords (3 out of 30) from the study. Second, as can be seen from the first three rows of Table 4.14, choosing stricter cut-off points $(0 \%, 1 \%$, or $5 \%)$ means a small number of learners either in total or in each group. In contrast, choosing a maximum of $10 \%$ error ensured that the present study had 112 learners in total with around 32 mid-low vocabulary level learners, and a good balance between the number of mid and high vocabulary level learners (40).

Third, the $10 \%$ figure still ensured that the Yes/ No test results were as reliable as the stricter cut-off points. Following Schmitt et al.'s (2011) approach, the current researcher conducted a series of independent-sample t-tests to compare the scores at the VLT of 88 learners who checked no more than $5 \%$ of the pseudowords, with that of the 112 
learners who checked no more than $10 \%$ of the pseudowords. The results showed no significant differences in the overall vocabulary levels score for the 5\% set $(M=$ $55.65 \%, S D=19.11)$, and the $10 \%$ set $(M=54.73, S D=18.38), t(198)=.344, p=0.731$ (2-tailed). Normality was confirmed using Kolmogorov-Smirnov test for normality ( $p>$ $0.05)$, and visually assessed using Q-Q plots and boxplots. Similar analysis with the $2 \mathrm{~K}$, $3 \mathrm{~K}$, and $5 \mathrm{~K}$ levels revealed the same results. These results suggested that choosing $5 \%$ or $10 \%$ did not make any difference in the VLT mean scores. Moreover, a comparison between the VLT scores of the 112 learners who checked no more than $10 \%$ of the pseudowords with the total number of target words they indicated were known in the Yes/ No tests revealed that there was a linear relationship between the two variables. In particular, there was a positive, strong, significant correlations between the learners' scores in the two tests $(r=.83)$. Given the high validity of the VLT, the strong correlation between the learners' scores in the two tests suggested that the data of the 112 learners who checked no more than $10 \%$ of pseudowords in the Yes/ No tests were accurate indicators of their vocabulary knowledge of the target words.

Table 4.14. Number of learners at different cut-off points of checked pseudowords

\begin{tabular}{lllllll}
\hline \multirow{2}{*}{$\begin{array}{c}\text { Checked pseudowords } \\
\%\end{array}$} & Raw & \multicolumn{2}{l}{ Number of learners in each group } & Number of \\
\cline { 3 - 5 } & Mid-low & Mid & High & Total & added learners \\
\hline 0 & 0 & 0 & 0 & 0 & 0 & 0 \\
1 & $0-5$ & 12 & 6 & 7 & 25 & 25 \\
5 & $0-24$ & 25 & 28 & 35 & 88 & 63 \\
10 & $0-48$ & 32 & 40 & 40 & 112 & 24 \\
\hline
\end{tabular}

Taken together, data of 112 learners checking no more than $10 \%$ of pseudowords (32 mid-low learners, 40 mid learners and 40 high learners) were used for the analysis which compared learner knowledge of the BNC/COCA2000 and New-GSL.

\subsubsection{Results of the Yes/ No tests}

Similar to the teacher surveys, data from the Yes/ No tests were looked at from two aspects: (1) the learners' knowledge of the 545 BNC/COCA words and 428 New-GSL words and (2) the most frequent 420 items from each list.

Table 4.15 presents the proportion of the BNC/COCA2000 and New-GSL words among the words known by $100 \%, 90 \%$, and $80 \%$ of the mid and high level learners. The 
second and third columns show that, when all 973 words were compared, the BNC/COCA2000 always accounted for a significantly larger proportion of words known by $100 \%, 90 \%$ and $80 \%$ of the learners (53.70\%-58.97\%) than the New-GSL (41.03\%-46.30\%). The last two columns present the percentage of each list that the learners knew when the most frequent 420 words from each list were compared. The BNC/COCA2000 accounted for a significantly larger proportion of words known by $100 \%$ of the learners. At the $90 \%$ and $80 \%$ levels, although the two lists took turns comprising higher proportions of known words, the differences were always insignificant at $p<0.05$. This means that it cannot be concluded whether the BNC/COCA2000 or the New-GSL had more words known by $90 \%$ and $80 \%$ of the learners.

Table 4.15. Proportion of the BNC/COCA2000 and New-GSL words among the words known by the mid and high groups $(N=80)(\%)$

\begin{tabular}{rrrrr}
\hline \% of learners & \multicolumn{2}{c}{ All 973 words } & \multicolumn{2}{c}{ The most frequent 420 words } \\
\cline { 2 - 5 } knowing the words & BNC/COCA2000 & New-GSL & BNC/COCA2000 & New-GSL \\
\hline $100 \%$ & 58.97 & 41.03 & 56.76 & 43.24 \\
$90 \%$ & 53.96 & 46.04 & 50.33 & 49.67 \\
$80 \%$ & 53.70 & 46.30 & 49.33 & 50.67 \\
\hline
\end{tabular}

When the supplementary data from the mid-low vocabulary learners were examined, whether all 973 target words or the most frequent 420 words from each list were examined, the BNC/COCA2000 always made up a significantly larger proportion of known words than the New-GSL, $p<0.05$ (Table 4.16).

Table 4.16. Proportion of the BNC/COCA2000 and New-GSL words among the words known by the mid-low group $(N=32)(\%)$

\begin{tabular}{rrrrr}
\hline \% of learners & \multicolumn{2}{c}{ All 973 words } & \multicolumn{2}{c}{ The most frequent 420 words } \\
\cline { 2 - 5 } knowing the words & BNC/COCA2000 & New-GSL & BNC/COCA2000 & New-GSL \\
\hline $100 \%$ & 68.42 & 31.58 & 69.44 & 30.56 \\
$90 \%$ & 67.36 & 32.64 & 66.17 & 33.83 \\
$80 \%$ & 65.46 & 34.54 & 62.67 & 37.33 \\
\hline
\end{tabular}

\subsubsection{Relationships between the corpus, teacher, and learner data}

Pearson product moment correlations were conducted to examine a possible relationship between lexical coverage, teacher perceptions of the usefulness, and learner vocabulary 
knowledge of each of the 973 target words. The interpretation of correlation values between 0 and 1 followed Cohen's (1988) guideline; that is, an $r$ value from 0.10 to 0.29 indicates a small correlation; from 0.30 to 0.49 a medium correlation, and 0.50 to 1.0 a large correlation. Hereafter, lexical coverage means the mean coverage provided by a headword in 18 corpora. The way to calculate the mean coverage was provided at the beginning of Section 4.4.1. Teacher perception of the word usefulness was the mean of the scores given by 78 teachers from all three groups for each word. Learner vocabulary knowledge was the proportion of learners knowing the words. It was calculated by dividing the number of learners knowing the word by the total number of learners who checked no more than $10 \%$ of the pseudowords.

\subsubsection{Relationship between lexical coverage and teacher perception of word usefulness}

When the data of all teachers were analyzed, a small but significant correlation was found between lexical coverage and teacher perception of word usefulness, $r=.23, n=$ 973, $p<0.0005$. Similar patterns were found when the data of each group of teachers were examined. There were always small and significant correlations between lexical coverage and teacher perception (Table 4.17). The correlations of the three groups of teachers are fairly similar with the $r$ value ranging from .21 to .23 .

Table 4.17. Correlation between lexical coverage and teacher perception of word usefulness

\begin{tabular}{lll}
\hline Groups of teachers & $\mathrm{N}$ & r correlation \\
\hline Vietnamese L1 teachers & 26 & $.23^{* *}$ \\
English L1 teachers & 25 & $.22^{* *}$ \\
Various L1 teachers & 27 & $.21^{* *}$ \\
\hline
\end{tabular}

**. Correlation is significant at the 0.01 level (2-tailed).

\subsubsection{Relationship between lexical coverage and learner vocabulary knowledge}

The analysis of the data from all learners revealed a small but significant correlation between the lexical coverage and learner vocabulary knowledge of the 973 target words, $r=.20, n=973, p<0.0005$. Similarly, when each group of learners was examined, the lexical coverage and learner vocabulary knowledge were significantly correlated at $p$ $<0.0005$ (Table 4.18). Of the four groups, the mid group had the higher correlation with lexical coverage. This may be because the vocabulary knowledge of this group is the 
most relevant to the vocabulary level that the target words represent (the most frequent 2,000 words). The correlations of the mid-low and high groups were slightly lower because the vocabulary levels of these learners were slightly lower or higher than the level that the target words represent. The low group had the lowest correlation, which is not surprising given the lack of vocabulary knowledge of these learners.

Table 4.18. Correlation between lexical coverage and learner vocabulary knowledge

\begin{tabular}{lll}
\hline Groups of learners & $\mathrm{N}$ & $\mathrm{r}$ correlation \\
\hline Mid & 40 & $.22^{* *}$ \\
Mid-low & 32 & $.19^{* *}$ \\
High & 40 & $.19^{* *}$ \\
Low & 133 & $.17^{* *}$ \\
\hline
\end{tabular}

\subsubsection{Relationship between learner vocabulary knowledge and teacher perception of word usefulness}

Table 4.19 presents the results of the examination between learner vocabulary knowledge and teacher perception. The last row and last column of the table indicates that there was a large correlation between learner vocabulary knowledge and teacher perception, $r=.67, n=973, p<.0005$. When data of each group of learners and each group of teachers were compared, similar patterns were found. The $r$ values ranged from .45 to .71 , which indicated medium to strong significant correlations. This means that the larger proportion of learners knowing the words, the higher the degree of usefulness of the words rated by the teachers.

Of the three groups of teachers, irrespective of the groups of learners being compared, Vietnamese L1 teachers always had the highest correlations ( $r$ from 0.58 to 0.71 ). Next came the various L1 teachers ( $r$ from 0.52 to 0.65 ). English L1 teachers had the lowest correlations ( $r$ from 0.45 to 0.56 ).

Of the four groups of learners, regardless of the teacher groups being compared, the mid and mid-low groups always had higher correlations with the teachers' ratings than the low and high groups. The correlation of the mid and mid-low groups were from .53 to .69 and from .55 to .70 , respectively while that of the other two groups were from .49 to .61 (low) and from .45 to .58 (high). 
Table 4.19. Correlation between learner vocabulary knowledge and teacher perception of word usefulness

\begin{tabular}{|l|l|l|l|l|l|l|}
\hline \multirow{2}{*}{\begin{tabular}{l}
\multirow{2}{*}{ Groups of teachers } \\
\cline { 3 - 7 }
\end{tabular}} & \multirow{2}{*}{ Low } & Mid-low & Mid & High & \multirow{2}{*}{ All } \\
\cline { 3 - 8 } & & $N=133$ & $N=32$ & $N=40$ & $N=40$ & $N=245$ \\
\hline Vietnamese L1 teachers & $N=26$ & $.61^{* *}$ & $.70^{* *}$ & $.69^{* *}$ & $.58^{* *}$ & $.71^{* *}$ \\
\hline Various L1 teachers & $N=27$ & $.52^{* *}$ & $.63^{* *}$ & $.65^{* *}$ & $.58^{* *}$ & $.62^{* *}$ \\
\hline English L1 teachers & $N=25$ & $.49^{* *}$ & $.55^{* *}$ & $.53^{* *}$ & $.45^{* *}$ & $.56^{* *}$ \\
\hline All & $N=78$ & $.57^{* *}$ & $.66^{* *}$ & $.66^{* *}$ & $.56^{* *}$ & $.67^{* *}$ \\
\hline
\end{tabular}

**. Correlation is significant at the 0.01 level (2-tailed).

\subsection{Summary of main findings}

This section summarizes the findings in this study. The findings related to the four research questions are presented first. They are then followed by other findings emerging from the results.

\subsubsection{The receptive vocabulary levels of Vietnamese EFL learners}

In answer to the first research question, the receptive vocabulary levels of the learner participants followed the typical lexical profile; that is, they knew more words at higher frequency levels than words at lower frequency levels. However, depending on their university years and majors, their scores on the VLT may vary from having not mastered the $1^{\text {st }} 1,000$ word level to having mastered the $2 \mathrm{~K}$ or even $3 \mathrm{~K}$ levels. Englishmajors tended to know more words than non-English majors. Senior students were more likely to know more words than junior students. Given this variation, the next study (Study 3) did not adopt a one-size-fit all benchmark of general vocabulary for every learner to acquire before learning items from the Academic Spoken Word List (ASWL). Instead, the ASWL was adaptable to learners' existing vocabulary levels.

The present study also found that, as a whole, the learner participants had not mastered any levels of the VLT even though they had spent an average of nine years studying English. Importantly, nearly $65 \%$ of the learners had not mastered the most frequent 2,000 word families, and more than half of the learners were unlikely to have mastered the most frequent 1,000 word families. In Chapter 6, these findings will be examined with the findings from other studies to discuss the suitable size of a general high- 
frequency word list for L2 learners (see Section 6.2.3) and how to make word lists adaptable to learners' proficiency (see Section 6.3.1).

\subsubsection{Teacher perception of word usefulness and learner knowledge of the BNC/COCA2000 and New-GSL words}

Research questions 2 and 3 are related to which list, the BNC/COCA2000 or the NewGSL, is the most suitable for L2 learners from the perspective of teachers and learners.

The second research question asks which list accounts for a larger proportion of headwords perceived as being useful for L2 learners by experienced teachers of English. Irrespective of the criteria of usefulness and groups of teachers, the BNC/COCA2000 consistently accounted for a larger proportion of useful words than the New-GSL. In most cases, the differences were significant. Importantly, as the criterion of usefulness got stricter, the differences became larger.

The third research question concerns which list accounts for a larger proportion of headwords known by $100 \%, 90 \%$, and $80 \%$ of the learners. When the non-overlapping headwords in the two lists were compared, the 545 items unique to the BNC/COCA2000 always made up a larger proportion of words known by learners than the 428 items unique to the New-GSL. In essence, the differences were significant in most cases. When the most frequent 420 items from each component of the target words (the $545 \mathrm{BNC} / \mathrm{COCA} 2000$ words and $428 \mathrm{New}-\mathrm{GSL}$ words) were compared, the BNC/COCA2000 had a significantly larger proportion of words know by $100 \%$ learners having mastered at least the most frequent 2,000 word families, and words known by $100 \%, 90 \%$, and $80 \%$ of the learners nearly mastered the most frequent 2000 word families.

Together these findings indicated that the BNC/COCA2000 is the most suitable general high-frequency word list for L2 learners, because (1) we should expect a general highfrequency word list to be made up of a large proportion of words considered to be useful by teachers, and (2) we should expect students to learn general high-frequency words early in their lexical development. Therefore, in the next study (Study 3), the BNC/COCA2000 was used to examine the coverage of general high-frequency words in academic spoken and written English. Moreover, the BNC/COCA lists, of which the $\mathrm{BNC} / \mathrm{COCA} 2000$ is a part, was chosen to guide the division of the ASWL into levels to match learners' existing vocabulary levels. The reason for the superiority of the 
BNC/COCA2000 over the other general high-frequency word lists will be explained in detail in Section 6.2.2. Also, in Chapter 6, these findings will be used to discuss what source of information should be used in word list validation (see Section 6.3.3).

\subsubsection{Relationship between lexical coverage, teacher perception, and learner vocabulary knowledge}

The last research question asks if there is a relationship between lexical coverage, teacher perception of word usefulness, and learner vocabulary knowledge. In answer to this question, these factors were significantly related to each other. However, there were differences in the degree of correlations between these factors. The correlations between lexical coverage and other two factors (vocabulary knowledge and teacher perception) were small. In contrast, there was a strong correlation between learner vocabulary knowledge and teacher perception. In Chapter 6, this finding will be drawn together with other findings from the three studies to discuss the sources of information needed for word list validation (see Section 6.3.3).

\subsubsection{The correlations of three groups of teachers with the learner vocabulary knowledge}

Apart from the findings related to the four research questions, one interesting finding that emerged from the results was the correlations in the ratings of each teacher group with learner vocabulary knowledge. The Vietnamese L1 teachers always had the highest correlations. Next came the various L1 teachers. The English L1 teachers had the lowest correlations. In Chapter 6, this finding will be discussed in detail to highlight the importance of teachers' familiarity with learners' characteristics in L2 vocabulary learning and teaching (see Section 6.4).

\subsection{Rationale for the next study}

In the first two studies of this thesis, lexical coverage, teacher perception of word usefulness, and learner vocabulary knowledge have been used to determine the most suitable general high-frequency word list for L2 learners. Also, these studies have investigated the relationship between the three factors. The next study then focuses particularly on the vocabulary in academic spoken English. It involves developing an ASWL, and using this list and Nation's (2012) BNC/COCA2000 as instruments to examine the nature of vocabulary in academic speech. 


\section{Chapter 5 - Study 3: Developing and validating an academic spoken word list}

\subsection{Introduction}

Studies 1 and 2 have used lexical coverage, learner knowledge, and teacher perceptions as criteria to compare four well-known general high-frequency word lists. They have indicated that the BNC/COCA2000 is the most suitable list for second language (L2) learners. The findings of Study 2 also suggest that the existing vocabulary levels of English for Academic Purposes (EAP) learners are varied. Therefore, it is important to develop an academic spoken word list which is adaptable to the learners' existing proficiency.

Study 3 explores the nature of vocabulary in academic speech. To be specific, it has three aims. The first aim is to identify which items appeared with high frequency in academic speech in a wide range of academic subjects and include them in an Academic Spoken Word List (ASWL). The list is then divided into levels according to Nation's (2012) BNC/COCA lists so that it can match learners' vocabulary levels. The second aim is to examine the nature of vocabulary in academic spoken English. This involves comparing the lexical coverage of the ASWL in academic spoken English, academic written English, and non-academic spoken English. The third aim is to determine the potential coverage that learners with different levels of general vocabulary may reach if they learn the ASWL. Study 3 seeks to shed light on the nature of vocabulary in academic spoken English. Also, it involves the development of an academic spoken word list that should be useful for EAP learners irrespective of their academic subject areas and proficiency levels.

Like the previous chapters, the remaining part of this chapter focuses on the research questions, methodology, results, and the main findings of Study 3. Section 5.2 presents the research question of the study. Sections 5.3 and 5.4 describe how the corpora used to develop and validate the ASWL were compiled while Section 5.5 justifies the unit of counting of the list. The establishment of the criteria for selecting the ASWL words is described in Sections 5.6 and 5.7. Sections 5.8 and 5.9 show how the data were analyzed. The results related to the five research questions are presented in Section 5.10. 
The chapter ends with a summary of the main findings (Section 5.11) and the rationale for the next chapter (Section 5.12).

\subsection{Research questions}

Study 3 was conducted to address six questions:

1. Which lexical items occur frequently and are evenly distributed in a wide range of academic speech?

2. Is there a difference in their coverage in academic speech in hard-pure, hardapplied, soft-pure, and soft-applied subjects?

3. What is the coverage of the ASWL in independent collections of academic speech, academic writing, and non-academic speech?

4. What is the coverage of Nation's (2012) BNC/COCA2000 in academic speech, academic writing, and non-academic speech?

5. What is the overlap between the ASWL with lists of general high-frequency words and academic written words?

6. With knowledge of the ASWL words, how much coverage of academic speech may be reached by learners with different levels of general vocabulary?

\subsection{Developing the two academic spoken corpora}

Four corpora of around the same size were developed in this study: two academic spoken corpora, one academic written corpus, and one non-academic spoken corpus. The first academic spoken corpus was used to create the ASWL while the other corpora were used to validate the list from different perspectives (see Table 5.1). This was the most common way of validating academic word lists in earlier research (Coxhead, 2000; Gardner \& Davies, 2014; Nation \& Webb, 2011).

The coverage of the ASWL in the second academic spoken corpus should indicate if the list accurately captures the high frequency, wide ranging, and evenly distributed word families in academic speech. Its coverage in the academic written corpus should determine whether the list better represents academic spoken vocabulary than academic written vocabulary. Likewise, its coverage in the non-academic spoken corpus would indicate if the ASWL accurately represents academic rather than non-academic vocabulary. Together, findings of this examination should suggest whether the ASWL is truly an academic spoken word list or not. 
Table 5.1. Academic and non-academic corpora in Study 3

\begin{tabular}{llc}
\hline Corpus & Purpose & $\begin{array}{c}\text { Size } \\
\text { (running-words) }\end{array}$ \\
\hline $1^{\text {st }}$ academic spoken corpus & Develop the ASWL & $13,029,661$ \\
$2^{\text {nd }}$ academic spoken corpus & $\begin{array}{l}\text { Determine if the ASWL accurately } \\
\text { reflects the vocabulary in academic } \\
\text { speech }\end{array}$ & $12,740,619$ \\
& $\begin{array}{l}\text { Examine if the ASWL reflects spoken } \\
\text { Academic written corpus }\end{array}$ & $13,449,584$ \\
vocabulary & $\begin{array}{l}\text { Examine if the ASWL reflects } \\
\text { Non-academic spoken corpus }\end{array}$ & $13,863,628$ \\
\hline
\end{tabular}

This section focuses on the development of the two academic spoken corpora. In his seminal paper, Biber (1993) suggested three steps that researchers should follow when designing a corpus so that their corpus can fully capture the variability of a language. They are (1) defining the target population represented by the corpus, (2) selecting the sampling frames and methods to represent the target population, and (3) evaluating the extent to which the final sample actually represents the target population. Moreover, as mentioned in Section 2.5.2.2, Coxhead $(1998,2000)$ provides comprehensive principles in developing an academic corpus. Therefore, Biber's (1993) and Coxhead's (1998, 2000) frameworks were used to guide every step of developing the two academic spoken corpora in this study so that these corpora represent as closely as possible the academic speech that the target users of the ASWL are likely to encounter in their study. This section begins with the selection of the materials for the two corpora. Then, it describes the development of the first academic spoken corpus (to develop the ASWL) and the second academic spoken corpus (to validate the list) in turn.

\subsubsection{Materials selection for the two academic spoken corpora}

The two academic spoken corpora in the present study were developed from materials recorded by lecturers and researchers rather than recorded by the current researcher 
herself. This approach was used to deal with the challenge of collecting and achieving large and representative academic spoken corpora. Unlike written texts which can be quickly collected in large amounts by scanning or downloading texts from the Internet, speech needs to be recorded, manually transcribed, and coded (McCarthy \& Carter, 1997; O’Keeffe, McCarthy, \& Carter, 2007; Thompson, 2006). Materials in the two academic spoken corpora came from 11 sources:

(1) six corpora from publishers

(2) four open access coursewares

(3) a collection of university lectures collected by an independent researcher

Table 5.2 presents an overview of these sources. The names of the courses from the open access coursewares are listed in Appendices 11 to 14. These materials represent naturally occurring academic spoken English in a wide range of institutions in different parts of the world and represent at least seven varieties of English. Of these sources, the six corpora from publishers were well-constructed for linguistic research purposes. Therefore, apart from sharing the same purpose with other sources, that is, to increase the size of the two academic spoken corpora in the present study, the use of these six corpora also allowed the current researcher to establish the principles that guided the construction of the two academic spoken corpora in this study. Let us now look at how the structure of the six corpora from publishers helped with the construction of the two academic spoken corpora in the present study from the perspectives of disciplinary division and speech events in turn.

\subsubsection{Disciplinary division in the two academic spoken corpora}

Table 5.3 provides an overview of the structure of the six corpora from publishers. The second column of the table shows that the disciplinary division in most corpora was based on the administrative structure of the institutions in which the data were recorded. Of the remaining corpora, one corpus, the Pearson International Corpus of Academic English (PICAE), relied on Wikipedia's classification while the other, British Academic Spoken English (BASE), adopted Becher's (1989) classification of academic disciplines in higher education to categorize their disciplinary sub-corpora. Following the developers of the BASE corpus, Becher's (1989) classification was used as a guide to organize the spoken materials from 11 sources into four disciplinary sub-corpora in the present study. According to this classification, academic disciplines can be divided into 
Table 5.2. Sources of the two academic spoken corpora in Study 3

\begin{tabular}{|c|c|c|c|c|c|c|c|}
\hline Source & Abbreviation & Location & Time & $\begin{array}{l}\text { Main varieties } \\
\text { of English }\end{array}$ & 1 st corpus & 2nd corpus & Total \\
\hline \multicolumn{8}{|l|}{ Corpora from publishers } \\
\hline $\begin{array}{l}\text { British Academic Spoken } \\
\text { English Corpus }\end{array}$ & BASE & $\begin{array}{l}\text { University of Warwick \& } \\
\text { University of Reading }\end{array}$ & $2000-2005$ & British & $1,090,040$ & 576,065 & $1,666,105$ \\
\hline $\begin{array}{l}\text { Michigan Corpus of } \\
\text { Academic Spoken English }\end{array}$ & MICASE & University of Michigan & $1997-2002$ & American & 707,287 & 400,205 & $1,107,492$ \\
\hline $\begin{array}{l}\text { Newcastle Corpus of } \\
\text { Academic Spoken English }\end{array}$ & NUCASE & University of Newcastle & 2011 & British & 407,370 & 124,214 & 531,584 \\
\hline $\begin{array}{l}\text { Limerick-Belfast Corpus of } \\
\text { Academic Spoken English }\end{array}$ & LIBEL & $\begin{array}{l}\text { University of Limerick \& } \\
\text { Queen's University Belfast }\end{array}$ & 2010-2011 & Irish & 181,047 & 295,180 & 476,227 \\
\hline $\begin{array}{l}\text { Pearson International Corpus } \\
\text { of Academic English } \\
\text { (Spoken component) }\end{array}$ & PICAE & $\begin{array}{l}\text { Longman Spoken } \\
\text { American Corpus/ } \\
\text { American National } \\
\text { Corpus/BNC }\end{array}$ & $2007-2010$ & $\begin{array}{l}\text { American, } \\
\text { Australian, } \\
\text { British, New } \\
\text { Zealand, } \\
\text { Canadian }\end{array}$ & 304,371 & 50,703 & 355,074 \\
\hline $\begin{array}{l}\text { Hong Kong Corpus of } \\
\text { Spoken English (academic) }\end{array}$ & HKCSE & A university in Hong Kong & $1997-2002$ & Hong Kong & 47,418 & 140,279 & 187,697 \\
\hline \multicolumn{8}{|l|}{ Open access coursewares } \\
\hline Yale University & & Yale University & 2006-2011 & American & $3,584,394$ & $3,455,485$ & $7,039,879$ \\
\hline $\begin{array}{l}\text { Massachusetts Institute of } \\
\text { Technology }\end{array}$ & & $\begin{array}{l}\text { Massachusetts Institute of } \\
\text { Technology }\end{array}$ & 2004-2014 & American & $2,323,068$ & $3,956,258$ & $6,279,326$ \\
\hline $\begin{array}{l}\text { University of California, } \\
\text { Berkeley }\end{array}$ & & $\begin{array}{l}\text { University of California, } \\
\text { Berkeley }\end{array}$ & $2011-2015$ & American & $3,142,428$ & $2,328,511$ & $5,470,939$ \\
\hline Stanford University & & Stanford University & $2007-2008$ & American & $1,043,367$ & $1,207,516$ & $2,250,883$ \\
\hline
\end{tabular}




\begin{tabular}{|c|c|c|c|c|c|c|c|}
\hline Source & Abbreviation & Location & Time & $\begin{array}{l}\text { Main varieties } \\
\text { of English }\end{array}$ & 1st corpus & 2nd corpus & Total \\
\hline \multicolumn{8}{|c|}{ Materials collected by an independent researcher } \\
\hline $\begin{array}{l}\text { Lectures at a university in } \\
\text { New Zealand }\end{array}$ & & $\begin{array}{l}\text { A university in New } \\
\text { Zealand }\end{array}$ & 2006-2009 & New-Zealand & 198,871 & 206,203 & 405,074 \\
\hline Total & & & & & $13,029,661$ & $12,740,619$ & $25,770,280$ \\
\hline
\end{tabular}

Table 5.3. Speech events and disciplinary division of the corpora from publishers

\begin{tabular}{|c|c|c|c|}
\hline \multirow[b]{2}{*}{ Source } & \multirow[b]{2}{*}{ Disciplinary division } & \multicolumn{2}{|c|}{ Speech events } \\
\hline & & Classroom events & Non-class events \\
\hline BASE & $\begin{array}{l}\text { Becher's (1989) classification of } \\
\text { academic disciplines at higher education }\end{array}$ & lectures \& seminars & None \\
\hline HKCSE & $\begin{array}{l}\text { administrative structure of a university in } \\
\text { Hong Kong }\end{array}$ & $\begin{array}{l}\text { lectures, seminars, student presentations, } \\
\text { tutorials, supervisions, \& workshops for staff }\end{array}$ & None \\
\hline LIBEL & $\begin{array}{l}\text { administrative structure of University of } \\
\text { Limerick and Queen's University Belfast }\end{array}$ & $\begin{array}{l}\text { lectures, seminars, tutorials, labs, conference } \\
\text { papers, \& EFL classes }\end{array}$ & None \\
\hline MICASE & $\begin{array}{l}\text { University of Michigan School of } \\
\text { Graduate Studies classification of } \\
\text { departments }\end{array}$ & $\begin{array}{l}\text { small lectures, large lectures, discussion } \\
\text { sections, seminars, student presentations, \& } \\
\text { labs }\end{array}$ & $\begin{array}{l}\text { advising sessions, colloquia, } \\
\text { dissertation defenses, interviews, } \\
\text { meetings, office hours, service } \\
\text { encounters, study groups, \& tours }\end{array}$ \\
\hline NUCASE & $\begin{array}{l}\text { administrative structure of University of } \\
\text { Newcastle }\end{array}$ & practicals, presentations, seminars & group work \& project meetings \\
\hline PICAE & Wikipedia's list of academic disciplines & lectures \& seminars & TV broadcasts \& radio broadcasts \\
\hline
\end{tabular}


four groups: hard-pure (e.g., Mathematics, Physics), hard-applied (e.g., Medicine, Engineering), soft-pure (e.g., History, Philosophy), and soft-applied (e.g., Business, Law). The hard/soft dimension is related to the degree to which a paradigm exists. The pure/ applied dimension associates with application to practical problems.

There were several reasons to use Becher's (1989) classification in this study. First, Becher's (1989) classification is highly valid. It was developed from Biglan's (1973a, 1973b) and Kolb's (1981) classification. Biglan (1973a, 1973b) examined the judgment of 219 scholars from various fields at different universities in the United States on the similarities among 36 academic disciplines. Based on the results, he suggested a threedimension, eight-cluster model that could reflect the characteristics of disciplinary subjects in higher education: hard-pure-nonlife, hard-applied-nonlife, hard-pure-life, hard-applied-life, soft-pure-non-life, soft-applied-nonlife, soft-pure-life, and softapplied-life. Kolb (1981), however, relied on the learning strategies of 800 postgraduates from a wide variety of disciplinary backgrounds to develop his classification. Despite the different approach, Kolb's (1981) results were surprisingly consistent with Biglan's (1973a, 1973b) studies. He found a strong similarity between his data on the learning style dimensions of abstract-concrete and active-reflective and Biglan's hard/soft, and pure/applied dimensions. As a result, Kolb (1981) suggested that a four-fold typology of disciplines could be developed based on these two dimensions: (1) the abstract-reflective (or hard-pure) quadrant, (2) the abstract-active (e.g., hardapplied) quadrant, (3) the concrete-active (e.g., soft-applied) quadrant, and (4) the concrete-reflective (soft-pure) quadrant. This classification later was adopted by Becher (1989) but was represented by Biglan's (1973a, 1973b) shorthand headings: hard-pure, soft-pure, hard-applied, and soft-applied. The validity of this classification has been confirmed by numerous empirical studies in different contexts (see Jones, 2011 for a review).

Second, the consistent results in studies validating Becher's (1989) classification in different contexts indicate that it can be transferable across institutions and can serve as a common standard for comparison (Nesi, 2002). As a result, it has been widely used as a way to categorize academic disciplines in higher education (see Jones, 2011; Neumann, 2001 for more details). In applied linguistics research, Becher's (1989) classification has been adopted to structure the BASE corpus, the British Academic Written English corpus (BAWE) corpus, and Hyland's $(2000,2004)$ academic written corpus. Given the high validity and wide transferability of Becher's (1989) 
classification, it is expected that the ASWL can be globally used by EAP learners irrespective of the administrative structure of their universities.

One limitation of Becher's (1989) classification is that it cannot reflect the complexity and variation in inquiry processes and knowledge structures in various disciplines due to the lack of clear-cut boundaries between hard/soft and pure/applied (Becher \& Trowler, 2001; Nesi, 2002). As a result, it may fail to classify interdisciplinary subjects and cannot capture the fact that, depending on the context, an individual course can range from hard to soft, or from pure to applied. This limitation, however, does not apply in the present study because its aim was to develop a universal academic spoken word list to suit the English for General Academic Purpose (EGAP) context, in which EAP learners plan to study different academic subjects or are not clear about their specific subject areas. In brief, because of its high validity and wide transferability, Becher's (1989) classification was chosen to categorize the disciplines in the two academic spoken corpora of the present study.

\subsubsection{Speech events represented in the two academic spoken corpora}

All six corpora represent academic spoken English as speech occurring in academic settings. However, as can be seen from the last two columns of Table 5.3 above, the way that academic settings are interpreted varies from corpus to corpus. The BASE corpus has the narrowest definition by restricting academic spoken English to lectures and seminars. The Hong Kong Corpus of Spoken English (HKCSE) and the LimerickBelfast Corpus of Academic Spoken English (LIBEL) also take a narrow view of academic spoken English, but expand to include other kinds of classroom speech events (labs, tutorials, student presentations, supervisions, workshops for staff, conference papers, and EFL classes). The PICAE, the Newcastle Corpus of Academic Spoken English (NUCASE), and the Michigan Corpus of Academic Spoken English (MICASE) have the broadest representation. In these corpora, academic spoken English is expanded to include language in non-class academic speech events.

The present study adopted the narrow view of academic spoken English; that is, classroom language, for two reasons. First, the target users of the ASWL are L2 learners who plan to study academic disciplines in English-medium programs. Considering these learners' limited time and their slow vocabulary growth rates (Milton, 2009; Webb \& Chang, 2012), focusing on classroom language is practical. It also better reflects these learners' primary needs; that is, being able to comprehend and engage in the academic courses with their instructors and fellow students. Compared with vocabulary in non- 
class academic speech events, vocabulary in classroom speech events comprises a larger proportion of subject-related vocabulary (Csomay, 2006) which is unlikely to be encountered often by learners in general conversation. In contrast, vocabulary in nonclass academic speech events may be a mixture of the vocabulary in classroom events and general conversation due to the overlap between non-classroom academic speech events within these domains. Thus, it is expected that it may not be too challenging for learners to comprehend non-class academic speech if they know classroom language and language of general conversation. Second, as mentioned in the previous section, the two academic spoken corpora in this study were divided into four disciplinary subcorpora: hard-pure, hard-applied, soft-pure, and soft-applied. Classroom language is more subject-focused than non-class language (Csomay, 2006). Therefore, focusing on classroom language makes it possible to classify the materials from the 11 sources into these sub-corpora.

Four classroom speech events are presented in the two academic spoken corpora of the present study: lectures, seminars, labs, and tutorials. Lectures are opportunities in which lecturers inform, evaluate, and critique important information in the reading materials that they would like to draw their students' attention to (Lynch, 2011). Although the degree of interaction between course instructors and students may vary according to lecture types, course instructors are the ones who mainly speak in this speech event. Seminars, tutorials, and labs are opportunities for students to participate in group discussion with lecturers, tutors, and fellow students (Adolphs \& Carter, 2013). According to Aguilar (2016), seminars can be classified into student instructional seminars and expert research seminars. Student instructional seminars refer to the involvement of interactions between course instructors and students. Course instructors act as the moderators who select the topic for investigation, but students are the ones who prepare the seminar discussion on the topic. Either instructors or students lead the discussion, but students are the ones who mainly speak. Expert research seminars, however, are opportunities for academics to speak about their on-going or completed research to a small expert audience. As the target users of the ASWL are L2 learners who plan to study their academic subjects at English-medium universities, in the present research, seminars are defined as student instructional seminars. Labs and tutorials are the speech events that provide students with opportunities to deepen their understanding of the concepts, theories, and reading issues mentioned in the lectures, as well as developing practical skills. Labs are more common in hard subjects while tutorials are more common in soft subjects (Neumann, 2001). Including labs and tutorials together 
with lectures and seminars ensures that the two academic spoken corpora better represent a range of academic speech that L2 students will often encounter at Englishmedium programs. It also better demonstrates the distinctive features of different disciplinary groups (hard versus soft). Other kinds of classroom speech events (supervision, workshops for staff, conference papers, and EFL classes) were not selected for the corpora in the present study. These events either focus more on staff than students (workshops for staff, conference papers), have limited application (supervision), or are not directly related to the academic disciplines that the learners will study at university (EFL classes). Taken together, four kinds of classroom speech events were represented in the two academic spoken corpora: lectures, seminars, labs, and tutorials.

\subsubsection{The first academic spoken corpus}

So far in this chapter, the selection of the materials for the two academic spoken corpora has been presented. This section describes how these materials were distributed into the first academic spoken corpus to develop the ASWL.

\subsubsection{Principles behind the corpus structure}

The development of the first academic spoken corpus was guided by the principles of size, representativeness, and balance. In terms of size, because the ASWL aims to benefit learners with different proficiency levels, the first academic spoken corpus should be large enough to capture a considerable number of occurrences of words outside general high-frequency words (Coxhead, 1998). Previous research has suggested that one-million running words per sub-corpus is sufficient for a spoken corpus (Baker, Hardie, \& McEnery, 2006; Brysbaert \& New, 2009; Nation, 2016). Hence, with its four sub-corpora, the first academic spoken corpus should have a total size of at least four-million running words.

In terms of representativeness, following Biber's (1993) and Coxhead's (2000) guidelines, the first academic spoken corpus should represent materials from a wide range of disciplines and subject areas. To be specific, this corpus would be divided into four sub-corpora following Becher's (1989) classification: hard-pure, hard-applied, softpure, and soft-applied. Each sub-corpus should be made up of materials from different subject areas. If possible, the proportion of lectures, seminar, labs, and tutorials in the corpus should reflect the proportion of these events encountered by the students in their academic study. That is, lectures should account for the largest proportion. Next come 
seminars, and then labs and tutorials depending on whether the subjects were hard or soft. This proportion was determined based on the fact that lectures and seminars are common speech events in the four disciplines while labs and tutorials are distinctive to hard and soft subjects, respectively. This was also supported by the analysis of the proportion of speech events in the six corpora from publishers as well as an analysis of the proportion of lectures, seminars, labs, and tutorials presented in the 2013 undergraduate and postgraduate course outlines at Victoria University of Wellington. Moreover, to maximize the representativeness of the corpus, when possible, materials from different courses should be prioritized over those from the same course, and materials represent different varieties of English should also be included. This helps to minimize the bias caused by speakers' styles and topics as well as a particular variety of English.

In terms of balance, the ASWL aims to benefit learners from a wide range of disciplines and subject areas. Hence, following Coxhead (2000), each disciplinary sub-corpus of the first academic spoken corpus should contain the same number of words and the same number of subject areas. Each subject area should have around the same number of words. This ensures that the corpus is not biased towards the vocabulary in a particular discipline or subject area.

Let us now look at how the first academic spoken corpus satisfied these principles. This corpus was comprised of 1,543 transcripts, which made up a total size of 13,029,661 running words. It is much larger than the suggested target size (four-million). It is more than eight times larger than the spoken corpus used to develop Nesi's (2002) Spoken Academic Word List (SAWL) (1.6-million) and more than six times larger than the spoken corpus used to create Simpson-Vlach and Ellis's (2010) spoken Academic Formulas List (AFL) (2.1-million). Each academic spoken corpus in the present study is even four times larger than the written corpus used to make Coxhead's (2000) Academic Word List (AWL) (3.5-million). Given the wide recognition of the AFL and AWL, it is expected that the two academic spoken corpora in this study are large enough to capture academic spoken vocabulary.

The transcripts came from all 11 sources and represent at least seven varieties of English (see Table 5.2 above). All four speech events are represented. Lectures account for the largest proportion. Next come seminars. Labs and tutorials account for the smallest proportion (see Table 5.4). 
Table 5.4. Speech events in the $1^{\text {st }}$ academic spoken corpus

\begin{tabular}{lrr}
\hline Speech events & Size (running words) & Proportion (\%) \\
\hline Lectures & $11,950,542$ & 91.72 \\
Seminars & 698,812 & 5.36 \\
Tutorials & 280,562 & 2.15 \\
Labs & 99,745 & 0.77 \\
\hline Total & $13,029,661$ & 100 \\
\hline
\end{tabular}

Following Coxhead (2000), the range in the corpus is looked at from two levels: the number of disciplinary sub-corpora and the number of subject areas in which the words appear. Table 5.5 illustrates the composition of the first academic spoken corpus. At the macro level, the corpus is divided into four sub-corpora: hard-pure, hard-applied, softpure, and soft-applied. Each sub-corpus contains around 3.25-million running words from around 380 transcripts, which is very even. This is the broadest level of organizing the corpus. It allows the current researcher to identify words common between disciplines and compare their occurrences in each discipline. At the micro level, each disciplinary sub-corpus is divided into six subjects. In other words, the first academic spoken corpus is comprised from 24 subject areas in total. The size of each subject is very similar (around 500,000 running words). The aim of this level is to enable the current researcher to identify the words appearing in a wide range of academic subjects that L2 learners are likely to study in English-medium programs.

Although the 11 sources contain materials from a larger number of hard-applied, softpure, and soft-applied subjects (see Section 5.3.1 above for these sources), six was chosen as the number of subjects in each sub-corpus of the first academic spoken corpus because only six hard-pure subjects are represented in these sources. This choice satisfied the balance principle; that is, the number of subjects in each sub-corpus of the first academic spoken corpus should be the same. Moreover, six subjects per sub-corpus is sufficient given the number of subjects in the academic corpora of previous research. It is much larger than the number of subjects per sub-corpus in Hyland and Tse's (2007) academic written corpus (two to three). Biber's (2006) Spoken and Written Academic Language Corpus also had six subjects per sub-corpus. Coxhead's (2000) academic 
Table 5.5. Composition of the $1^{\text {st }}$ academic spoken corpus

\begin{tabular}{|c|c|c|c|c|c|c|c|}
\hline Hard-pure & & Hard-applied & & Soft-pure & & Soft-applied & \\
\hline 379 transcripts & & 388 transcripts & & 406 transcripts & & 370 transcripts & \\
\hline Subject & Size & Subject & Size & Subjects & Size & Subjects & Size \\
\hline Astronomy & 593,062 & Chemical Engineering & 563,938 & Art & 553,160 & Business & 513,133 \\
\hline Biology & 552,452 & Computer Sciences & 555,175 & Cultural Studies & 498,393 & Economics & 610,998 \\
\hline Chemistry & 556,138 & Cybernetics & 555,401 & History & 554,214 & Education & 571,023 \\
\hline Ecology \& Geology & 555,312 & Electrical Engineering & 550,181 & Philosophy & 549,577 & Law & 616,398 \\
\hline Mathematics & 450,481 & Health \& Medical Sciences & 470,795 & Political Studies & 545,059 & Management & 461,093 \\
\hline Physics & 554,178 & Mechanical Engineering & 558,604 & Psychology & 555,880 & Public Policy & 485,016 \\
\hline Total & $3,261,623$ & Total & $3,254,094$ & Total & $3,256,283$ & Total & $3,257,661$ \\
\hline
\end{tabular}


written corpus also had around the same number of subjects per sub-corpus (seven).

Additionally, although six subjects are represented in the sub-corpora of the first academic spoken corpus to develop the ASWL, the list was evaluated against a second academic spoken corpus which contains a wider range of subjects (42), more than $50 \%$ of which do not appear in the first academic spoken corpus (see Section 5.3.4 below for the description of this corpus). The following section describes each sub-corpus of the first academic spoken corpus in detail.

\subsubsection{Hard-pure sub-corpus of the first academic spoken corpus}

The hard-pure sub-corpus contains 3,261,623 words from 379 transcripts. Table 5.6 demonstrates the composition of this sub-corpus. Three types of speech events are represented. Lectures account for the largest proportion and then seminars. Labs make up the smallest proportion of this sub-corpus. There are no materials from tutorials because they are not common in hard subjects. Except for Mathematics, which has 450,481 running words, the size of the other subjects is around 550,000 words.

Table 5.6. Composition of the hard-pure sub-corpus ( $1^{\text {st }}$ academic spoken corpus)

\begin{tabular}{lrrrr}
\hline Subject & Lectures & Seminars & Labs & Total \\
\hline Astronomy & 593,062 & 0 & 0 & 593,062 \\
Chemistry & 497,423 & 50,634 & 8,081 & 556,138 \\
Ecology \& Geology & 555,312 & 0 & 0 & 555,312 \\
Physics & 554,178 & 0 & 0 & 554,178 \\
Biology & 496,159 & 25,553 & 30,740 & 552,452 \\
Mathematics & 435,715 & 8,170 & 6,596 & 450,481 \\
\hline Total size & $3,131,849$ & 84,357 & 45,417 & $3,261,623$ \\
Proportion (\%) & 96.02 & 2.59 & 1.39 & 100 \\
\hline
\end{tabular}

\subsubsection{Hard-applied sub-corpus of the first academic spoken corpus}

The hard applied sub-corpus was derived from 388 transcripts which make up a total size of 3,254,094 running words. Table 5.7 shows that, similar to the hard-pure sub-corpus, three types of speech events (lectures, seminars, and labs) are represented in the hard-applied sub-corpus with lecturing accounting for the largest proportion and labs for the smallest. Each subject consists of over 550,000 running words, except for the Health and Medical Sciences whose size is 470,795 running words. 
Table 5.7. Composition of the hard-applied sub-corpus ( $1^{\text {st }}$ academic spoken corpus)

\begin{tabular}{lrrrr}
\hline Subject & Lectures & Seminars & Labs & Total \\
\hline Chemical Engineering & 535,243 & 9958 & 18737 & 563,938 \\
Mechanical Engineering & 558,604 & 0 & 0 & 558,604 \\
Cybernetics & 555,401 & 0 & 0 & 555,401 \\
Computer Sciences & 535,852 & 6,990 & 12,333 & 555,175 \\
Electrical Engineering & 550,181 & 0 & 0 & 550,181 \\
Health \& Medical Sciences & 338,629 & 132,166 & 0 & 470,795 \\
\hline Total size & $3,073,910$ & 149,114 & 31,070 & $3,254,094$ \\
Proportion (\%) & 94.46 & 4.58 & 0.95 & 100 \\
\hline
\end{tabular}

\subsubsection{Soft-pure sub-corpus of the first academic spoken corpus}

The soft-pure sub-corpus includes 3,256,283 running words from 406 transcripts. Unlike the two hard sub-corpora, this sub-corpus includes materials from all four speech events (see Table 5.8). Lectures comprise the largest proportion of the sub-corpus, then seminars, and tutorials. Labs have the smallest proportion. The proportion of the four speech events reflects the nature of soft subjects; that is, tutorials are more common than labs in soft subjects. Except for Cultural Studies which has 498,393 words, the other subjects contain around 550,000 words.

Table 5.8. Composition of the soft-pure sub-corpus ( $1^{\text {st }}$ academic spoken corpus)

\begin{tabular}{lrrrrr}
\hline Subject & Lectures & Seminars & Labs & Tutorial & Total \\
\hline Psychology & 532,622 & 0 & 23258 & 0 & 555,880 \\
History & 554,214 & 0 & 0 & 0 & 554,214 \\
Art & 512,991 & 28869 & 0 & 11,300 & 553,160 \\
Philosophy & 515,767 & 33810 & 0 & 0 & 549,577 \\
Political Studies & 498,652 & 19,347 & 0 & 27,060 & 545,059 \\
Cultural Studies & 395,396 & 102,997 & 0 & 0 & 498,393 \\
\hline Total size & $3,009,642$ & 185,023 & 23,258 & 38,360 & $3,256,283$ \\
Proportion (\%) & 92.43 & 5.68 & 0.71 & 1.18 & 100 \\
\hline
\end{tabular}

\subsubsection{Soft-applied sub-corpus of the first academic spoken corpus}

The soft-applied sub-corpus, which was derived from 370 transcripts, consists of 3,257,661 running words. The composition of this sub-corpus is presented in Table 5.9. Three speech events are represented in this sub-corpus: lectures, seminars, and tutorials. Lectures make 
up the largest proportion of the corpus. Tutorials comprise the smallest proportion. Labs are not represented because they are not common in soft subjects.

Table 5.9. Composition of the soft-applied sub-corpus $\left(1^{\text {st }}\right.$ academic spoken corpus)

\begin{tabular}{lrrrr}
\hline Subject & Lectures & \multicolumn{1}{l}{ Seminars } & Tutorial & Total \\
\hline Law & 607,500 & 8898 & 0 & 616,398 \\
Economics & 604,083 & 6,915 & 0 & 610,998 \\
Education & 484,668 & 29,713 & 56,642 & 571,023 \\
Business & 178,080 & 149493 & 185,560 & 513,133 \\
Public Policy & 430,339 & 54677 & 0 & 485,016 \\
Management & 430,471 & 30,622 & 0 & 461,093 \\
\hline Total size & $2,735,141$ & 280,318 & 242,202 & $3,257,661$ \\
Proportion (\%) & 83.96 & 8.60 & 7.43 & 100 \\
\hline
\end{tabular}

Unlike the other three sub-corpora, the number of running words in the soft-applied subcorpora is slightly uneven. Law and Economics each has more than 600,000 running words. In contrast, Management and Public Policy each have nearly 500,000 running words due to the lack of materials. However, the evenness in the size of the soft-applied subjects should have little effect on the ASWL because the difference in the sizes of the subjects is fairly small, and the total size of the soft-applied sub-corpus is around the same as the other subcorpora. Moreover, the loss of important items is minimized by the fact that words selected for the ASWL should occur in a wide range of subjects from the four disciplinary subcorpora combined.

\subsubsection{The second academic spoken corpus}

The previous section has described the development and structure of the first academic spoken corpus, which was used to develop the ASWL. This section focuses on the development of the second academic spoken corpus, in which the list was validated. In general, the principles behind the structure of this corpus are similar to those used to structure the first academic spoken corpora, but they were applied with more flexibility due to the difficulty of collecting academic spoken materials. 
Table 5.10. Composition of the $2^{\text {nd }}$ academic spoken corpus

\begin{tabular}{|c|c|c|c|c|c|c|c|}
\hline $\begin{array}{l}\text { Hard-pure } \\
361 \text { transcripts }\end{array}$ & & $\begin{array}{l}\text { Hard-applied } \\
299 \text { transcripts }\end{array}$ & & $\begin{array}{l}\text { Soft-pure } \\
416 \text { transcripts }\end{array}$ & & $\begin{array}{l}\text { Soft-applied } \\
284 \text { transcripts }\end{array}$ & \\
\hline Subjects & Size & Subjects & Size & Subject & Size & Subject & Size \\
\hline Biology & 699,286 & Applied Statistics $^{*}$ & 14,179 & Anthropology* & 53,903 & Architecture \& Design ${ }^{*}$ & 103,417 \\
\hline Chemistry & 761,025 & Civil Engineering* $^{*}$ & 33,718 & Archeology* & 10,382 & Economics & $1,907,942$ \\
\hline Ecology \& Geology & 15,459 & Computer Sciences & 347,348 & Art & 204,759 & Education & 63,910 \\
\hline Mathematics & 924,437 & Construction ${ }^{*}$ & 20,358 & Classic Studies ${ }^{*}$ & 290,367 & Film, Theater, Music* & 71,022 \\
\hline \multirow[t]{13}{*}{ Physics } & 768,409 & Cybernetics & 904,854 & Communication $^{*}$ & 124,335 & Law & 895,181 \\
\hline & & Electrical Engineering & $1,581,306$ & $\begin{array}{l}\text { Cultural Studies } \\
\text { English \& }\end{array}$ & 345,588 & Nursing ${ }^{*}$ & 119,903 \\
\hline & & Engineering Graphics* & 22,409 & Literature $^{*}$ & 220,456 & Textiles \& Clothing ${ }^{*}$ & 13,829 \\
\hline & & $\begin{array}{l}\text { General Engineering* } \\
\text { Industrial \& Operation }\end{array}$ & 118,751 & Gender Studies* & 34,451 & & \\
\hline & & Engineering $^{*}$ & 10,722 & Geography* & 204,806 & & \\
\hline & & Manufacturing* & 63,912 & History & 782,628 & & \\
\hline & & Marine Engineering* & 46,567 & Journalism* & 34,463 & & \\
\hline & & Mechanical Engineering & 21,279 & Linguistics* & 86,692 & & \\
\hline & & Meteorology ${ }^{*}$ & 43,439 & Philosophy & 65,370 & & \\
\hline & & & & Political Science & 67,401 & & \\
\hline & & & & Psychology & 28,181 & & \\
\hline & & & & Religious Studies* & 398,652 & & \\
\hline & & & & Sociology ${ }^{*}$ & 215,523 & & \\
\hline Total & $3,168,616$ & Total & $3,228,842$ & Total & $3,167,957$ & Total & $3,175,204$ \\
\hline
\end{tabular}

* Subjects that do not appear in the $1^{\text {st }}$ academic spoken corpus 
Table 5.10 presents the composition of the second academic spoken corpus. To some extent, this corpus is a mirror of the first academic spoken corpus. Like the first corpus, the second corpus was derived from around 1,400 transcripts which made up a total size of about 13-million running words. All four kinds of speech events were represented in this corpus. Lectures comprise the largest proportion. Next come seminars. Labs and tutorials account for the smallest proportions (see Table 5.11 for the proportion of speech events in the second academic spoken corpus).

Table 5.11. Size of the four speech events in the $2^{\text {nd }}$ academic spoken corpus

\begin{tabular}{lrr}
\hline Speech event & Size (running words) & Proportion (\%) \\
\hline Lectures & $12,304,666$ & 96.58 \\
Seminars & 285,625 & 2.24 \\
Tutorials & 112,844 & 0.89 \\
Labs & 374,84 & 0.29 \\
\hline Total & $12,740,619$ & 100 \\
\hline
\end{tabular}

The corpus is divided into four equally-sized disciplinary sub-corpora: hard-pure, hardapplied, soft-pure, and soft-applied. Each sub-corpus consists of around 3.2-million running words. The difference between the two academic spoken corpora is that the second corpus represents a larger number of subjects (42), more than half of which are not represented in the first corpus. Moreover, the number of subjects per sub-corpus in the second academic spoken corpus and the number of words in these subjects are more varied (see Table 5.10). The following section will provide a detailed description of each sub-corpus of the second academic spoken corpus in turn.

\subsubsection{Hard-pure sub-corpus of the second academic spoken corpus}

The hard-pure sub-corpus contains 3,168,616 running words. This sub-corpus is comprised of 361 transcripts, which are all lectures. As can be seen in the first column of Table 5.10 above, five subjects are represented in this sub-corpus. They are Biology, Chemistry, Ecology and Geology, Mathematics, and Physics. All of these subjects were included in the first academic spoken corpus. The sizes of these subjects in the second academic spoken corpus ranged from 15,459 (Ecology \& Geology) to 924,437 running words (Mathematics). 


\subsubsection{Hard-applied sub-corpus of the second academic spoken corpus}

The hard-applied sub-corpus was developed from 299 transcripts from 13 subject areas that make up a total size of 3,228,842 running words. Nine of these subjects did not appear in the first academic spoken corpus. Table 5.12 shows the composition of this sub-corpus.

Table 5.12. Composition of the hard-applied sub-corpus (2 $2^{\text {nd }}$ academic spoken corpus)

\begin{tabular}{|c|c|c|c|c|c|}
\hline Subject & Lectures & Seminars & Labs & Tutorials & Total \\
\hline Electrical Engineering & $1,581,306$ & 0 & 0 & 0 & $1,581,306$ \\
\hline Cybernetics & 904,854 & 0 & 0 & 0 & 904,854 \\
\hline Computer Sciences & 347,348 & 0 & 0 & 0 & 347,348 \\
\hline General Engineering* & 47,033 & 20,114 & 13,090 & 38,514 & 118,751 \\
\hline Manufacturing* & 56,573 & 7,339 & 0 & 0 & 63,912 \\
\hline Marine Engineering* & 46,567 & 0 & 0 & 0 & 46,567 \\
\hline Meteorology ${ }^{*}$ & 35,070 & 8,369 & 0 & 0 & 43,439 \\
\hline Civil Engineering* & 23,684 & 0 & 10,034 & 0 & 33,718 \\
\hline Engineering Graphics ${ }^{*}$ & 22,409 & 0 & 0 & 0 & 22,409 \\
\hline Mechanical Engineering & 21,279 & 0 & 0 & 0 & 21,279 \\
\hline Construction $^{*}$ & 4,664 & 15,694 & 0 & 0 & 20,358 \\
\hline Applied Statistics ${ }^{*}$ & 14,179 & 0 & 0 & 0 & 14,179 \\
\hline Industrial \& Operation Engineering* & 10,722 & 0 & 0 & 0 & 10,722 \\
\hline Total size & $3,115,688$ & 51,516 & 23,124 & 38,514 & $3,228,842$ \\
\hline Proportion (\%) & 96.50 & 1.60 & 0.72 & 1.19 & 100 \\
\hline
\end{tabular}

${ }^{*}$ Subjects that do not appear in the $1^{\text {st }}$ academic spoken corpus

All four speech events are represented. Lectures account for the largest proportion of the sub-corpus, and then are followed by seminars. Labs and tutorials comprise the smallest proportion. The sizes of the subjects represented in the sub-corpus range from 10,711 to $1,577,752$ running words.

\subsubsection{Soft-pure sub-corpus of the second academic spoken corpus}

The soft-pure sub-corpus had 416 transcripts from 17 subjects, which comprise a total size of 3,167,957 running words. Table 5.13 shows that all four speech events are represented. Their order in terms of size is lectures, seminars, tutorials, and labs. Of the 17 subjects, 11 subjects do not appear in the first academic spoken corpus. 
Table 5.13. Composition of the soft-pure sub-corpus ( $2^{\text {nd }}$ academic spoken corpus)

\begin{tabular}{|c|c|c|c|c|c|}
\hline Subject & Lectures & Seminars & Labs & Tutorial & Total \\
\hline History & 777,039 & 0 & 0 & 5,589 & 782,628 \\
\hline Religious Studies* & 398,652 & 0 & 0 & 0 & 398,652 \\
\hline Cultural Studies & 323,864 & 21,724 & 0 & 0 & 345,588 \\
\hline Classic Studies* & 290,367 & 0 & 0 & 0 & 290,367 \\
\hline English \& Literature* & 126,239 & 93,208 & 0 & 1,009 & 220,456 \\
\hline Sociology ${ }^{*}$ & 215,523 & 0 & 0 & 0 & 215,523 \\
\hline Geography* & 204,806 & 0 & 0 & 0 & 204,806 \\
\hline Art & 204,759 & 0 & 0 & 0 & 204,759 \\
\hline Communication $^{*}$ & 104,338 & 5,258 & 8687 & 6,052 & 124,335 \\
\hline Linguistics $^{*}$ & 63,606 & 23,086 & 0 & 0 & 86,692 \\
\hline Political Science & 57,531 & 0 & 0 & 9,870 & 67,401 \\
\hline Philosophy & 51,829 & 13,541 & 0 & 0 & 65,370 \\
\hline Anthropology ${ }^{*}$ & 53,903 & 0 & 0 & 0 & 53,903 \\
\hline Journalism $^{*}$ & 34,463 & 0 & 0 & 0 & 34,463 \\
\hline Gender Studies* & 21,323 & 13,128 & 0 & 0 & 34,451 \\
\hline Psychology & 21,251 & 6,930 & 0 & 0 & 28,181 \\
\hline Archeology ${ }^{*}$ & 10,382 & 0 & 0 & 0 & 10,382 \\
\hline Total size & $2,959,875$ & 176,875 & 8,687 & 22,520 & $3,167,957$ \\
\hline Proportion (\%) & 93.43 & 5.58 & 0.27 & 0.71 & 100 \\
\hline
\end{tabular}

${ }^{*}$ Subjects that do not appear in the $1^{\text {st }}$ academic spoken corpus

\subsubsection{Soft-applied sub-corpus of the second academic spoken corpus}

The soft-applied sub-corpus consists of 3,175,204 running words from 284 transcripts. The composition of the corpus is presented in Table 5.14. There are seven subjects in the subcorpus. Four of them (Nursing, Architecture \& Design, Film, Theater, Music, and Textiles $\&$ Clothing) do not appear in the first academic spoken corpus. The number of running words in the subjects ranges from 13,829 to 1,907,942 running words. All four speech events are represented in this sub-corpora. Most of the materials are transcripts of lectures. Seminars account for the second largest proportion. Next come tutorials. Labs make up the smallest proportion of the sub-corpus. 
Table 5.14. Composition of the soft-applied sub-corpus ( $2^{\text {nd }}$ academic spoken corpus)

\begin{tabular}{llllll}
\hline Subject & Lectures & Seminars & Labs & Tutorial & Total \\
\hline Economics & $1,907,942$ & 0 & 0 & 0 & $1,907,942$ \\
Law & 895,181 & 0 & 0 & 0 & 895,181 \\
Nursing $^{*}$ & 104,314 & 15,589 & 0 & 0 & 119,903 \\
Architecture \& Design $^{*}$ & 83,115 & 20,302 & 0 & 0 & 103,417 \\
Film, Theater, Music $^{*}$ & 44,006 & 21,343 & 5,673 & 0 & 71,022 \\
Education & 19,482 & 0 & 0 & 44,428 & 63,910 \\
Textiles \& Clothing $^{*}$ & 6,447 & 0 & 0 & 7,382 & 13,829 \\
\hline Total size & $3,060,487$ & 57,234 & 5,673 & 51,810 & $3,175,204$ \\
Proportion (\%) & 96.39 & 1.8 & 0.18 & 1.63 & 100 \\
\hline
\end{tabular}

* Subjects that do not appear in the $1^{\text {st }}$ academic spoken corpus

\subsection{Developing the academic written corpus and non-academic spoken corpus}

So far in this chapter, the development of the two academic spoken corpora has been described. This section presents the development of the academic written corpus and nonacademic spoken corpus.

The academic written corpus was developed to test whether the ASWL reflects spoken vocabulary. Table 5.15 shows that this corpus includes the reading materials in 30 courses at a university in New Zealand and 29 open courses delivered by Massachusetts Institution of Technology (MIT) (see Appendix 15 for the names of the MIT courses). It also contains proficient assessed writings from the BAWE corpus, which was downloaded from the Oxford Text Archive (http://ota.ahds.ac.uk), and the academic texts of science (2010-2012) in Corpus of Contemporary American-English (COCA). Together, these components make up a corpus of 13,449,584 running words. The corpus represents different kinds of academic writing (book chapters, journal articles, student writings, research reports, and textbooks) as well as three varieties of English (American-English, British-English, and New Zealand-English). As can be seen in Table 5.16, this corpus has a similar structure as the two academic spoken corpora. It is divided into four disciplinary sub-corpora, each of which contains more than 3-million running words. Each sub-corpus contains a range of subject areas. 
Table 5.15. Sources of the academic written corpus

\begin{tabular}{llr}
\hline Component & Course location & Size \\
\hline Academic section of the COCA corpus ( science, 2010-2012) & U.S.A & $1,195,124$ \\
Reading materials from courses in a New Zealand university & New-Zealand & $2,929,247$ \\
Reading materials in MIT open courseware & U.S.A & $2,988,387$ \\
BAWE corpus & U.K & $6,336,826$ \\
\hline Total & & $13,449,584$ \\
\hline
\end{tabular}

Table 5.16. Composition of the academic written corpus in terms of disciplines

\begin{tabular}{lrlrlrrr}
\hline \multicolumn{1}{c}{ Hard pure } & & \multicolumn{2}{c}{ Hard applied } & \multicolumn{2}{c}{ Soft pure } & \multicolumn{2}{l}{ Soft applied } \\
\hline Subjects & Words & Subjects & Words & Subjects & Words & Subjects & Words \\
Astronomy & 293,720 & Agriculture & 425,647 & Anthropology & 110,084 & Architecture & 20,449 \\
Biology & 341,250 & Civil engineering & 430,706 & Archeology & 184,828 & Business & 319,167 \\
Chemistry & 122,283 & Computer science & 191,735 & Classic Studies & 201,195 & Economics & 214,940 \\
Ecology \& Geology & 275,173 & Cybernetics & 86,208 & Cultural Studies & 211,260 & Education & $1,249,258$ \\
General Sciences & $1,195,124$ & Electrical engineering & 576,810 & English & 262,155 & Law & 405,044 \\
Mathematics & 688,465 & General Engineering & 720,587 & History & 286,184 & Management & 738,946 \\
Physics & 183,776 & Health \& Medicine & 398,153 & Linguistics & 253,306 & Public Policies & 56,479 \\
& & Material Engineering & 155,905 & Philosophy & 247,281 & \\
& & Mechanical engineering & 382,337 & Political Studies & $1,585,357$ & \\
& & Media Art \& Science & 120,796 & Psychology & 195,616 & \\
\hline Total & & Meteorology & 42,728 & Sociology & 276,632 & \\
\hline
\end{tabular}


Table 5.17. Non-academic spoken corpus

\begin{tabular}{llr}
\hline Corpus & Main variety of English & Size \\
\hline International Corpus of English (spoken, non-academic) & \multicolumn{1}{l}{$\begin{array}{l}\text { Indian, Pilipino, Singapore, } \\
\text { Canadian, Hong Kong, Irish, }\end{array}$} \\
$\begin{array}{ll}\text { Jamaican \& New Zealand } \\
\text { Open American National Corpus (spoken, non-academic) }\end{array}$ & American & $3,243,302$ \\
Webb \& Rodgers's (2009a) movie corpus & British \& American & $2,841,573$ \\
Hong Kong Corpus of Spoken English (non-academic) & Hong Kong & 760,516 \\
TV program corpus (Rodgers \& Webb, 2011) & British \& American & 943,110 \\
London-Lund corpus & British & 512,801 \\
Santa Barbara Corpus of Spoken American-English (non-academic) & American & 299,822 \\
\hline Total & & $13,863,628$ \\
\hline
\end{tabular}


The non-academic spoken corpus was developed to examine if the ASWL reflects academic vocabulary. This corpus has a similar size $(13,863,628$ running words) to the academic spoken corpora and the academic written corpus. Table 5.17 reveals that the nonacademic spoken corpora was comprised from the non-academic components of seven spoken corpora used in Study 1 (Chapter 3). They represent different kinds of general spoken English and 10 varieties of English. More information about these corpora can be found in Table 3.1 in Section 3.3.2.

\subsection{Determining the unit of counting for the ASWL}

The previous section described the development of the four corpora used in the present study, this section focuses on the unit of counting for the ASWL. It begins with the justification for the choice of word families at Bauer and Nation's (1993) Level 6. Then it describes how Level 6 word families in the first academic spoken corpus were identified.

\subsubsection{Justifying the unit of counting}

Word families up to Bauer and Nation's (1993) Level 6 were chosen as the primary unit of counting for the ASWL for two reason. First, this is the most commonly used unit of counting in earlier studies of academic word lists (Coxhead, 2000; Nesi, 2002; Xue \& Nation, 1984). Choosing Level 6, therefore, makes it possible to compare the results of the present study with previous research. Second, ideally, what is included in the word family should increase according to the growth of learners' morphological proficiency, but, in reality, making word family lists is very time consuming (Nation, 2016). Together with the analysis from computer programs, a large amount of manual checking and editing is required to ensure that derivational affixes are correctly classified into word families. The target users of the ASWL are from different learning contexts, and therefore, it will be very challenging to create word list versions at various word family levels to satisfy the needs of every group of learners. As Level 6 is nearly the broadest level of word families, choosing this level as the unit of counting for the ASWL may deal with the diversity in the characteristics of the list users to some extent. Importantly, following Coxhead (2000) and Nation (2013), the use of word families at Level 6 in this study is looked at from a pedagogical perspective. That is, knowledge of word family members is gradually picked

up during the learning process rather than acquired all at the same time. Knowledge of one word family member helps to facilitate the acquisition of other members. This assumption 
is supported by the results of a number of studies showing that L2 learners' derivational knowledge increases incrementally over time (Mochizuki \& Aizawa, 2000; Schmitt \& Meara, 1997; Schmitt \& Zimmerman, 2002). In other words, a Level 6 word family list should be seen as a guide in vocabulary learning rather than a handbook for learners and teachers to strictly follow.

However, Level 6 word families may overestimate the morphological knowledge of a proportion of English as a Foreign Language (EFL) learners (Brown, 2013; Mochizuki \& Aizawa, 2000; Schmitt \& Meara, 1997; Schmitt \& Zimmerman, 2002; Ward \& Chuenjundaeng, 2009; Webb \& Sasao, 2013). Therefore, apart from the list of Level-6 word family, to make the ASWL adaptable to learners' proficiency levels, two other versions of the ASWL are being developed. One version listed the Level 2.5 word families, and the other listed Level 3 word families (see Appendix 16 for samples of word family members in these versions). Low-level learners can choose the ASWL version that best suits their proficiency.

\subsubsection{Identifying the word families in the first academic spoken corpus}

The Level 6 word families in the first academic corpus were created with the aid of Nation's (2012) 25,000 BNC/COCA word families, as well as the word families in Nation's (2012) lists of proper nouns, marginal words, compound words, and abbreviations. These word lists were downloaded from the BNC/COCA25000 RANGE package available at Paul Nation's website. There were some special cases in the determination of the word families in the first academic spoken corpus. These cases are listed below.

\subsubsection{Transparent compounds}

Transparent compounds (e.g., textbook, keyword) are words which are comprised from two existing words, and the meaning of the words are closely related to the meaning of its part (Nation, 2016). There are three approaches dealing with these words. The first approach is to consider them as separate word families and list them in a separate list. This is the approach taken in the development of Nation's (2012) BNC/COCA lists. A second approach is to break the transparent compounds into parts so that each part is counted as a separate word (Brysbaert, Stevens, Mandera, \& Keuleers, 2016). For example, textbook is split into two words text and book, and their occurrences are counted along with the use of the component words (text, book). A third approach is to consider transparent compounds 
as members of the least frequent component of the compounds (Nation, 2016; Nation \& Webb, 2011). For instance, in the first academic spoken corpus of the present study, text (freq $=1,148)$ is less frequent than book (freq=4,613). Following the third approach means listing textbook under the word family text. The occurrences of textbook are then added to the occurrence of the word family text.

The third approach was chosen as the way to deal with transparent compounds in this study because it better serves the purpose of the study than the other two options. This study aimed to develop a word list that is adaptable to learners' existing vocabulary levels. Following the first approach by considering transparent compounds as separate words may overestimate the learning burden of these words. It does not take learners' knowledge of the components of compounds into account and may lead to the risk of including known compounds in the ASWL levels that are beyond learners' existing vocabulary knowledge. This then results in repeatedly teaching and learning known items. In contrast, the second approach may underestimate the learning burden of transparent compounds. If this approach had been adopted, the compound forms would have been lost. In other words, compounds would not have appeared in the ASWL either as family headwords or members. As a result, the learners may not see the importance of these words. For example, following this approach textbook will not appear in ASWL.

The third approach offers a compromise between the first and second approaches. Listing the compounds as members of the less frequent component takes the learning burden of compound words into account. For instance, learners without knowledge of the less frequent component of the compounds (text) may not be able to infer the meaning of the compounds (textbook) even if they know the meaning of the more frequent component (book). However, if they know the meaning of the less frequent component, this knowledge together with the knowledge of the more frequent component may enable these learners to recognize the meaning of the compounds. This way will ensure that the ASWL does not include the compounds, both components of which are already known by learners, meanwhile, include compounds which consist of at least one unknown component. For this reason, the third approach was adopted to deal with compound words in the present study. 


\subsubsection{Word types outside the BNC/COCA lists}

Other special cases are word types appearing in the first academic corpus that were outside the BNC/COCA lists. They were 2,294 proper nouns (e.g., Brocklin, Antonio, Furhmann), 32 marginal words (e.g., hahahhah, arrrgh), and 2,130 abbreviation representing formulas (e.g., $\mathrm{CH}_{3} \mathrm{CH}_{2} \mathrm{CH}_{2} \mathrm{OH}, \mathrm{CH}_{3} \mathrm{COOH}$ ). Also, they included 267 word types occurring more than 50 times in the first academic corpus but were neither proper nouns, marginal words, nor abbreviations (e.g., orbiters, coriolis). The proper nouns, marginal words, and abbreviations were added to the relevant BNC/COCA lists. The way to deal with the 267 word types which were neither proper nouns, marginal words, nor abbreviations was as follows.

First, the meanings of the word types were looked up in dictionaries and on the Internet. The results revealed that all of them were real words. The second step involved considering the meaning relationship of the types and the potential word families of which it may be a member. If they were related, the affixes of the word types were then checked in Bauer and Nation's (1993) taxonomy of affixes. If the affixes appeared in Bauer and Nation's (1993) scale up to Level 6, the types were listed as members of the word families. For instance, orbiter was included in the word family orbit because they were related in meaning, and the affix er appeared at Level 3 of Bauer and Nation's (1993) scale. In contrast, if the meanings of the word types and the potential word family were not related, or the affixes of the types were not in Bauer and Nation' (1993) scale up to Level 6, the word types and their potential word families were treated as separate word families. For example, coriolis was treated as a separate word family because there were no BNC/COCA word families having a related meaning with this type. Transcontinental was considered as a separate word family from continent even though they are related in meaning. This was because the affix trans was not listed in Bauer and Nation's (1993) scale up to Level 6.

In the third step, the current researcher consulted two hard-pure, four hard-applied, one soft-pure and two soft-applied postgraduate students to deal with 62 word types that the current researcher did not know. These word types include 20 hard-pure subject words (e.g., orthonormal), 23 hard-applied subject words (e.g., glomerulus), 12 soft-pure subject words (e.g., tholos), and seven soft-applied subject words (e.g., bailout). Depending on their disciplinary areas, these students were asked to look through the relevant lists and indicate if the words existed in their disciplines. They were also explained the definition of 
word families, and asked if certain words (e.g., subspace, space) were related to each other. For the words that the current researcher could not find possible family members, these students were asked to brainstorm any possible words they knew that came from the same word family. Based on their opinions, the treatment to the 267 word types that did not appear in the BNC/COCA25000 lists were adjusted. Consequently, 180 word types were added to existing BNC/COCA word families (e.g., subspace, sorter) whereas 87 word families were treated as separate word families and were included in a separate list (e.g., glomerulus, nephron).

\subsubsection{Calculating the range, frequency and dispersion of the word families in the first academic spoken corpus}

As mentioned in Section 5.6 below, range, frequency, and dispersion were chosen as the criteria to select the ASWL words. Therefore, an important step was to identify the range, frequency, and dispersion of the word families in the first academic spoken corpus. The range and frequency figures were calculated by Nation, Heatley and Coxhead's (2002) RANGE program. The dispersion figures were calculated by following two steps. First, the relative frequency of the word families per 100,000 items was calculated. Normalized frequency was used so that Juilland and Chang-Rodríguez's (1964) dispersion can be applied for unequally sized sub-corpus. For example, the relative frequency of be in the Physics subject was 6,701.10. It is calculated by dividing the raw frequency by the number of words in the Physics subject and multiplying by $100,000(37,136 \div 554,178 \times 100,000)$. Second, Juilland and Chang-Rodrigues's (1964) dispersion D for each word family was calculated by using Brezina's (2014) Statistics Toolbox which is available at Lancaster University website.

This section has justified the choice of Level 6 word families as the unit of counting for the ASWL. Also, it has described how the word families in the first academic spoken corpus were identified and how their range, frequency, and dispersion were calculated. The following section presents the principles behind the development of the ASWL and how these principles guided the selection of the ASWL words.

\subsection{Establishing the characteristics of the ASWL}

The ASWL must possess the following four characteristics: 
(1) Size and coverage: The ASWL must contain a smaller number of word families but provide higher coverage in the first academic spoken corpus than the BNC/COCA2000.

(2) Word families outside general high-frequency word families: The ASWL must include a considerable number of word families outside the BNC/COCA2000, but have high frequency, wide range, and even distribution in the first academic spoken corpus.

The purpose of the ASWL is to direct EAP learners' attention to the most important words in academic speech that are beyond their existing vocabulary levels. Therefore, if the ASWL either had a larger size but provided lower coverage than the BNC/COCA2000 or contained mainly the BNC/COCA2000 words, it would not draw much interest from EAP learners and teachers. They may simply use the BNC/COCA2000 rather than putting their effort on learning a new list.

(3) Distribution across the four sub-corpora: The coverage of the ASWL in the subcorpora of the first academic spoken corpus should be similar. In this way, the ASWL can benefit a wide range of EAP learners irrespective of their disciplines.

(4) Adaptability to learners' levels: the ASWL must be divided into four levels according to Nation's (2012) BNC/COCA lists to benefit learners with different proficiency levels (see Table 5.18). Levels 1-3 contain ASWL word families from the $1^{\text {st }}, 2^{\text {nd }}$, and $3^{\text {rd }} 1,000 \mathrm{BNC} / C O C A$ frequency levels, respectively. Level 4 represents ASWL items that are outside the most frequent 3,000 BNC/COCA word families.

Table 5.18. Four levels of the ASWL

\begin{tabular}{ll}
\hline ASWL level & Relevant BNC/COCA level \\
\hline Level 1 & $1^{\text {st }} 1,000$ \\
Level 2 & $2^{\text {nd }} 1,000$ \\
Level 3 & $3^{\text {rd }} 1,000$ \\
Level 4 & Outside the most frequent 3,000 words \\
\hline
\end{tabular}


ASWL words at Level 4 were not further classified. Learners with a vocabulary size of 4,000 word families may be able to recognize $95 \%$ of the words in academic speech (Dang \& Webb, 2014; Webb \& Paribakht, 2015), which may enable them to achieve a high and stable degree of listening comprehension (van Zeeland \& Schmitt, 2013). Therefore, perhaps learning the ASWL words that are outside their existing vocabulary levels is not as crucial for learners having mastered the most frequent 4,000 BNC/COCA word families as for those with lower levels of general vocabulary. Moreover, as was mentioned in Section 2.8.3 of the Literature review chapter, the proportion of general high-frequency words in academic speech is higher than in academic writing. Hence, it is expected that the number of ASWL word families beyond the most frequent 4,000 BNC/COCA word families would be very small, and their coverage would not be meaningful enough. However, in order for the ASWL to be also useful for learners with the vocabulary levels higher than the most frequent 4,000 BNC/COCA word families, the ASWL also provides information about the BNC/COCA levels at which the ASWL Level 4 word families appear.

To make sure that the list benefits learners at different vocabulary levels, not only the whole list but its levels should have the first three characteristics. This section has justified the characteristics that the ASWL must have. The following section describes how these characteristics influenced the decisions related to the range, frequency and dispersion criteria in the selection of the ASWL words.

\subsection{Determining the criteria for selecting the ASWL words}

A selected ASWL word family had to satisfy the range, frequency, and dispersion criteria. These criteria have been widely used to make corpus-based word lists so that lists capture items that occur frequently and distribute evenly in a wide range of target texts (Nation, 2016; Nation \& Webb, 2011). Another common criterion in research on developing academic word lists is specialized occurrence; that is the academic words should be outside general high-frequency words (Browne, Culligan, \& Phillips, n.d.; Campion \& Elley, 1971; Coxhead, 2000; Nesi, 2002; Praninskas, 1972; Xue \& Nation, 1984). This criterion was not used to select the ASWL because the present study aims to develop an ASWL that is 
adaptable to learners' vocabulary levels. The range, frequency, and dispersion criteria of the ASWL are as follows.

(1) Range: a selected word family had to occur in all four disciplinary sub-corpora (hard-pure, hard-applied, soft-pure, and soft-applied) of the first academic spoken corpus, and at least $50 \%$ of the subject areas (12 out of 24 subjects).

(2) Frequency: a selected word family had to occur at least 350 times in the first academic spoken corpus.

(3) Dispersion: a selected word family had to have Juilland and Chang-Rodríguez's (1964) dispersion D of at least 0.6 across 24 subjects.

Let us look at the justification for these criteria in turn.

\subsubsection{Setting the ASWL range criterion}

The range criterion (a selected word family had to occur in all sub-corpora and at least 50\% of the subject areas) was chosen because it has been consistently used in studies aimed at developing specialized word lists (Coxhead, 2000; Coxhead \& Hirsh, 2007; Hsu, 2013; Khani \& Tazik, 2013; Mungra \& Canziani, 2013; Valipouri \& Nassaji, 2013; Wang, Liang, \& Ge, 2008; Yang, 2015). It ensures that students from a wide range of disciplines can benefit from learning the ASWL. The frequency and dispersion criteria were established based on the result of extensive experimentation which compared the items included or excluded from the ASWL at different frequency cut-off points. The following sections explain more details how the ASWL frequency and dispersion criteria were established in the present study.

\subsubsection{Setting the ASWL frequency criterion}

The frequency cut-off point of 350 was chosen as a result of a comparison of eight pilot lists. These lists satisfied the ASWL range and dispersion criteria mentioned above. The only difference between these lists is the frequency criterion. The lists represented eight different frequency cut-off points ranging from 50 to 370 . For instance, the pilot list with the frequency cut-off point of 370 contains word families with the frequency of 370 or higher.

The 370 figure was transferred from the frequency criterion used by Coxhead (2000) to select the AWL words. In her study, the selected word families had to occur at least 100 
times in the 3.5-million running word academic written corpus. This criterion has been consistently adopted by subsequent specialized written word list studies with adjustment to the size of their academic corpora (Coxhead \& Hirsh, 2007; Hsu, 2013; Khani \& Tazik, 2013; Mungra \& Canziani, 2013; Valipouri \& Nassaji, 2013; Wang et al., 2008; Yang, 2015). The first academic spoken corpus in the present study $(13,029,661$ running words) is 3.7 times larger than Coxhead's (2000) academic written corpus (3,513,330 running words). Applying Coxhead's (2000) frequency criterion means that the selected ASWL word families had to occur at least 370 times in the first academic spoken corpus.

Frequency cut off points higher than 370 were not examined because, as mentioned in Section 2.8.3, general high-frequency words accounted for a larger proportion of texts in academic speech than in academic writing. Because the 370 figure was the frequency criterion for an academic written list, the difference in the vocabulary between the spoken and written modalities suggested that frequencies higher than 370 would have resulted in an ASWL which was mainly made up of general high-frequency words. If so, the second characteristic of an ASWL mentioned in Section 5.6 (word families outside general highfrequency word families) would not be satisfied.

The comparison showed that, of the eight frequency cut-off points, 350 satisfied the four characteristics of an ASWL best. First, it better fulfilled the first characteristic of an ASWL (size and coverage) mentioned in Section 5.6 than lists with the frequency cut-off points of 250 or lower. As can be seen from Table 5.19, the pilot list with the frequency cut-off point of 350 had fewer items than the most frequent 2,000 BNC/COCA word families. In contrast, although the pilot lists with frequency cut-off points of 250 or lower also provided higher coverage than the most frequent 2,000 BNC/COCA word families, they all contained more than 2,000 items. In other words, unlike the cut-off points of 250 or lower, 350 resulted in a word list which had fewer items but provided higher coverage in the first academic spoken corpus and its sub-corpora than the most frequent 2,000 BNC/COCA word families. 
Table 5.19. Coverage of the pilot lists with frequency cut-off points from 50 to 350 in the $1^{\text {st }}$ academic spoken corpus (\%)

\begin{tabular}{lrrrrrr}
\hline List (frequency cut-off points) & $\begin{array}{r}\text { Number of } \\
\text { word }\end{array}$ & $\begin{array}{r}\text { Whole } \\
\text { families }\end{array}$ & $\begin{array}{r}\text { Hard } \\
\text { corpus }\end{array}$ & $\begin{array}{r}\text { Hard } \\
\text { applied }\end{array}$ & $\begin{array}{r}\text { Soft } \\
\text { pure }\end{array}$ & $\begin{array}{r}\text { Soft } \\
\text { applied }\end{array}$ \\
\hline 50 & 3,416 & 92.10 & 91.01 & 92.70 & 91.60 & 93.07 \\
100 & 2,827 & 91.77 & 90.76 & 92.43 & 91.15 & 92.74 \\
150 & 2,487 & 91.47 & 90.51 & 92.18 & 90.71 & 92.40 \\
200 & 2,223 & 91.12 & 90.25 & 91.91 & 90.26 & 92.00 \\
250 & 2,033 & 90.79 & 90.00 & 91.66 & 89.80 & 91.63 \\
300 & 1,878 & 90.45 & 89.73 & 91.37 & 89.40 & 91.30 \\
$\mathbf{3 5 0}$ & $\mathbf{1 , 7 4 1}$ & $\mathbf{9 0 . 1 3}$ & $\mathbf{8 9 . 4 6}$ & $\mathbf{9 1 . 0 7}$ & $\mathbf{8 9 . 0 0}$ & $\mathbf{9 0 . 9 2}$ \\
\hline $1^{\text {st }}$ and $2^{\text {nd }} 1,000$ BNC/COCA & 2,000 & 88.61 & 87.37 & 88.76 & 88.51 & 89.83 \\
\hline
\end{tabular}

Second, the frequency cut-off point of 350 resulted in a list which satisfied the fourth characteristic of an ASWL (Adaptability to learners' levels) mentioned in Section 5.6 better than the frequency cut-off point of 300. The list with the frequency cut-off point of 350 is more beneficial for learners who have mastered the most frequent 1,000 words than the pilot list with the frequent cut-off point of 300. Because the learners already know the most frequent 1,000 words, words from the $1^{\text {st }} 1,000 \mathrm{BNC} / \mathrm{COCA}$ frequency level were excluded from these pilot lists. Table 5.20 shows that both lists provided higher coverage than the $2^{\text {nd }}$ 1,000 BNC/COCA word families. Yet, the list with the frequency cut-off point of 350 had fewer items than the $2^{\text {nd }} 1,000 \mathrm{BNC} / \mathrm{COCA}$ word families. In contrast, the list with the frequency cut-off point of 300 had more items.

Table 5.20. Coverage of the pilot lists with frequency cut-off points of 350 and 300 in the $1^{\text {st }}$ academic spoken corpus (excluding Level 1) (\%)

\begin{tabular}{lrrrrrr}
\hline List (frequency) & $\begin{array}{r}\text { Number of } \\
\text { word families }\end{array}$ & $\begin{array}{r}\text { Whole } \\
\text { corpus }\end{array}$ & $\begin{array}{r}\text { Hard } \\
\text { pure }\end{array}$ & $\begin{array}{r}\text { Hard } \\
\text { applied }\end{array}$ & $\begin{array}{r}\text { Soft } \\
\text { pure }\end{array}$ & $\begin{array}{r}\text { Soft } \\
\text { applied }\end{array}$ \\
\hline 300 & 1,024 & 8.77 & 8.78 & 9.14 & 8.03 & 9.12 \\
$\mathbf{3 5 0}$ & $\mathbf{9 1 1}$ & $\mathbf{8 . 5 1}$ & $\mathbf{8 . 5 5}$ & $\mathbf{8 . 8 8}$ & $\mathbf{7 . 7 1}$ & $\mathbf{8 . 8 2}$ \\
\hline $2^{\text {nd }} 1,000$ BNC/COCA & 1,000 & 6.33 & 6.01 & 6.10 & 6.33 & 6.89 \\
\hline
\end{tabular}


Third, compared with the frequency cut-off point of 370, 350 offered a better compromise between the second characteristic (Word families outside general high-frequency word families) and third characteristic of the ASWL (Distribution across the four sub-corpora). Table 5.21 presented the $S D$ of the coverage of the two pilot lists (350 and 370) in the four sub-corpora of the first academic spoken corpus. The $S D$ figures indicated the degree of evenness in the distribution of the two lists across the four sub-corpora. The smaller the $S D$ was, the more evenly the list is distributed.

Table 5.21. Difference in the coverage provided by the pilot lists with frequency cut-off points of 350 and 370 in four sub-corpora of the $1^{\text {st }}$ academic spoken corpus (SD)

\begin{tabular}{llllll}
\hline List (frequency) & Whole list & Level 1 & Level 2 & Level 3 & Level 4 \\
\hline $\mathbf{3 5 0}$ & $\mathbf{1 . 0 4}$ & $\mathbf{0 . 6 2}$ & $\mathbf{0 . 2 9}$ & $\mathbf{0 . 2 5}$ & $\mathbf{0 . 2 8}$ \\
370 & 1.05 & 0.63 & 0.29 & 0.25 & 0.27 \\
\hline
\end{tabular}

It can be seen that the difference in the $S D$ of the two pilot lists was small. The two lists took turns having higher $S D$ and had the same $S D$ in some cases. This finding indicates that, despite its lower frequency criterion, the pilot list with the frequency cut-off point of 350 satisfied the third characteristic of the ASWL (Distribution across the four subcorpora) as well as the frequency cut-off point of 370 .

However, lowering the frequency cut-off point from 370 to 350 means adding 23 word families outside the most frequent 2,000 BNC/COCA word families to the ASWL. These words families came from a wide range of BNC/COCA word levels (from the $3^{\text {rd }}$ to $7^{\text {th }}$ 1,000-word level) (Table 5.22), and most of them occurred in at least 20 out of 24 subjects and had a dispersion of 0.7 or above (Table 5.23). Setting 370 as the frequency cut-off point means the absence of these 23 high frequency, wide ranging, and evenly distributed word families from the ASWL. In other words, compared with 370, 350 better satisfied the second characteristic of an ASWL (Word families outside general high-frequency word families). It can be seen that, compared with the cut-off point of 370, 350 still ensured that the ASWL was evenly distributed across the sub-corpora, meanwhile, including more high frequency, wide ranging, and evenly distributed items outside the most frequent 2,000 BNC/COCA word families. 
Table 5.22. Twenty-three word families beyond the most frequent 2,000 BNC/COCA word families added when the frequency cut-off point moved from 370 to 350

\begin{tabular}{lrl}
\hline BNC/COCA level & Number of words & Words \\
\hline $3^{\text {rd }} 1,000$ & 16 & $\begin{array}{l}\text { perception, beam, principal, contradict, } \\
\text { excess, trend, differ, client, inject, approve, }\end{array}$ \\
& & journal, fragment, formation, amend, \\
& & eastern, cluster \\
& 3 & infer, scenario, tutor \\
$4^{\text {th }} 1,000$ & 2 & saturate, analytic \\
$5^{\text {th }} 1,000$ & 1 & micro \\
$6^{\text {th }} 1,000$ & 1 & subtract \\
$7^{\text {th }} 1,000$ & & \\
\hline
\end{tabular}

Table 5.23. Range and dispersion of the 23 word families beyond the most frequent 2,000 BNC/COCA word families added when the frequency cut-off point moved from 370 to 350

\begin{tabular}{rr|rr}
\hline \multicolumn{2}{c|}{ Range } & \multicolumn{2}{c}{ Dispersion } \\
\hline Number of subjects & Number of word families & D & Number of word families \\
\hline 24 & 3 & $0.8-0.9$ & 6 \\
23 & 3 & $0.7-0.8$ & 10 \\
22 & 6 & $0.6-0.7$ & 7 \\
21 & 3 & & \\
20 & 3 & & \\
19 & 2 & & \\
18 & 2 & & \\
16 & 1 & & \\
\hline
\end{tabular}

Taken as a whole, the ASWL with a frequency cut-off point of 350 best satisfied the four characteristics listed in Section 5.6. Unlike lower frequency cut-off points, 350 resulted in a word list that had a smaller number of items but provided higher coverage in the first academic spoken corpus and its sub-corpora. Unlike the higher cut-off point, 350 resulted in the inclusion of more high frequency, wide ranging, and evenly distributed items outside general high-frequency words. Meanwhile, it still ensured a similar degree of evenness in relation to the frequency cut-off point of 370 . The frequency cut-off point of the ASWL is lower than that applied to academic written word lists in earlier research, which reflects the difference between the nature of academic speech and writing. 


\subsubsection{Setting the ASWL dispersion criterion}

Dispersion indicates the evenness of the distribution of a word family across the corpus. The dispersion value can range from 0 (extremely uneven distribution) to 1 (perfectly even distribution). Similar to the frequency criterion, the dispersion cut-off point of 0.6 is the result of an examination which compared 10 different pilot versions using different dispersion cut-off points. It should be noted that the 10 pilot lists used in this section were different from the eight pilot lists in Section 5.7.2 above.

The 10 pilot lists in this section all satisfied the range and frequency criteria mentioned at the beginning of Section 5.7. The only difference between these pilot lists was the dispersion measure. Nine pilot lists represented nine different Juilland and ChangRodríguez's (1964) dispersion cut-off points ranging from 0.1 to 0.9 . For example, the pilot list with the dispersion cut-off of 0.6 contained words with a dispersion of at least 0.6 across the 24 subjects. One pilot list used Coxhead's (2000) uniformity as the criterion; that is, a word family had to occur at least 37 times in each sub-corpus of the first academic spoken corpus. The 37 figure was transferred from Coxhead's (2000) uniformity criterion. In her study, a selected AWL word family had to occur 10 times in each sub-corpus of Coxhead's (2000) written academic corpus. As the first academic spoken corpus in the present study is 3.7 times larger than Coxhead's corpus, if Coxhead's uniformity were applied, a word family would have to occur at least 37 in each sub-corpus of the first academic spoken corpus. Juilland and Chang-Rodríguez's (1964) dispersion was used in the comparison because this measure has received increasing attention from research on developing specialized word lists (Coxhead \& Hirsh, 2007; Gardner \& Davies, 2014; Lei \& Liu, 2016). Moreover, this dispersion measure has been widely used in corpus-based research to develop general word lists (Nation, 2004, 2012). Coxhead's (2000) uniformity criterion was used for the comparison because it is the most common measure of dispersion in previous research on developing specialized word lists (Khani \& Tazik, 2013; Valipouri $\&$ Nassaji, 2013; Ward, 2009). The comparison revealed that, of the 10 pilot lists, the list with the dispersion cut-off point of 0.6 better satisfied the four characteristics of the ASWL than other lists.

First, the pilot list with the dispersion cut-off point of 0.6 fulfilled the first characteristic of an ASWL (Size and coverage) better than those with the dispersion cut-off points of 0.1 and 0.2. Table 5.24 showed that, although all three pilot lists provided higher coverage than 
the most frequent 2,000 BNC/COCA word families, the list with the dispersion cut-off point of 0.6 contained much fewer items than the most frequent 2,000 BNC/COCA word families. In contrast, lists with the dispersion cut-off points of 0.1 and 0.2 had more items. It means that, compared with dispersion cut-off points of 0.1 and $0.2,0.6$ resulted in a word list which had fewer items but provided higher coverage than the most frequent 2,000 BNC/COCA word families.

Table 5.24. Coverage of the pilot lists with D of 0.1, 0.2 and 0.6 in the $1^{\text {st }}$ academic spoken corpus (\%)

\begin{tabular}{lrccccc}
\hline \multirow{2}{*}{ List (dispersion) } & $\begin{array}{l}\text { Number of } \\
\text { word families }\end{array}$ & $\begin{array}{l}\text { Whole } \\
\text { corpus }\end{array}$ & $\begin{array}{l}\text { Hard } \\
\text { pure }\end{array}$ & $\begin{array}{l}\text { Hard } \\
\text { applied }\end{array}$ & $\begin{array}{l}\text { Soft } \\
\text { pure }\end{array}$ & $\begin{array}{l}\text { Soft } \\
\text { applied }\end{array}$ \\
\hline 0.1 & 2,023 & 92.38 & 92.31 & 93.51 & 90.73 & 92.97 \\
0.2 & 2,016 & 92.35 & 92.27 & 93.47 & 90.7 & 92.97 \\
$\mathbf{0 . 6}$ & $\mathbf{1 , 7 4 1}$ & $\mathbf{9 0 . 1 3}$ & $\mathbf{8 9 . 4 6}$ & $\mathbf{9 1 . 0 7}$ & $\mathbf{8 9 . 0}$ & $\mathbf{9 0 . 9 2}$ \\
\hline $1^{\text {st }} \& 2^{\text {nd }} 1,000 \mathrm{BNC} / \mathrm{COCA}$ & 2,000 & 88.61 & 87.37 & 88.76 & 88.51 & 89.83 \\
\hline
\end{tabular}

Second, the pilot list with the dispersion cut-off point of 0.6 better satisfied the third characteristic (Distribution across the four sub-corpora) and the fourth characteristic (Adaptability to learners' levels) than the pilot lists with dispersion cut-off point of $0.3,0.4$, 0.5, 0.7, and Coxhead's (2000) uniformity. Table 5.25 presents the degree of evenness in distribution of these pilot lists and their levels cross the sub-corpora. The evenness was measured by the $S D$ of the coverage. The lower the $S D$ was, the more even the distribution of the list across the first academic spoken corpus was.

Table 5.25. Difference in the coverage in the $1^{\text {st }}$ academic spoken corpus provided by each level of the pilot lists with D of 0.3, 0.4, 0.5, 0.6, 0.7, and Coxhead's uniformity (SD)

\begin{tabular}{llllll}
\hline List (dispersion) & Whole list & Level 1 & Level 2 & Level 3 & Level 4 \\
\hline 0.3 & 1.16 & 0.66 & 0.37 & 0.32 & 0.72 \\
0.4 & 1.14 & 0.64 & 0.38 & 0.30 & 0.59 \\
0.5 & 1.09 & 0.60 & 0.33 & 0.28 & 0.45 \\
$\mathbf{0 . 6}$ & $\mathbf{1 . 0 4}$ & $\mathbf{0 . 6 2}$ & $\mathbf{0 . 2 9}$ & $\mathbf{0 . 2 5}$ & $\mathbf{0 . 2 8}$ \\
0.7 & 1.10 & 0.72 & 0.35 & 0.24 & 0.06 \\
Coxhead's uniformity & 1.28 & 0.63 & 0.36 & 0.56 & 0.12 \\
\hline
\end{tabular}


Table 5.26 demonstrates the ranking of these six pilot lists in terms of evenness according to the $S D$ figures. Number 1 indicates the list with the most even distribution while number 6 refers to the list with the least even distribution. Except for the case of Level 4, in all cases, the pilot list with the dispersion cut-off point of 0.6 either ranked first or second in terms of even distribution. The other lists either had lower or unstable ranking, or both.

Table 5.26. Ranking in term of even distribution in the $1^{\text {st }}$ academic spoken corpus of the pilot lists with D of 0.3, 0.4, 0.5, 0.6, 0.7, and Coxhead's uniformity

\begin{tabular}{llllll}
\hline Lists (dispersion) & Whole list & Level 1 & Level 2 & Level 3 & Level 4 \\
\hline 0.3 & 5 & 5 & 5 & 5 & 6 \\
0.4 & 4 & 4 & 6 & 4 & 5 \\
0.5 & 2 & 1 & 2 & 3 & 4 \\
$\mathbf{0 . 6}$ & $\mathbf{1}$ & $\mathbf{2}$ & $\mathbf{1}$ & $\mathbf{2}$ & $\mathbf{3}$ \\
0.7 & 3 & 6 & 3 & 1 & 1 \\
Coxhead's uniformity & 6 & 3 & 4 & 6 & 2 \\
\hline
\end{tabular}

Third, the pilot list of 0.6 met the second characteristic of an ASWL (Word families outside general high-frequency word families) better than the pilot lists with the dispersion cut-off point of 0.8 and of 0.9 . The pilot list with the dispersion cut-off point of 0.6 had 455 words families outside the BNC/COCA2000. Their coverage in the first academic spoken corpus was $3.28 \%$ (whole corpus) and 2.63\%-3.64\% (sub-corpora). In contrast, the pilot list with the dispersion cut-off point of 0.8 only had 154 word families outside the BNC/COCA2000, which covered only $1.19 \%$ of the $1^{\text {st }}$ academic spoken corpus and $1.16 \%-1.25 \%$ of its sub-corpora. Thus, lowering the dispersion cut-off point from 0.8 to 0.6 would add a further 301 words families beyond the most frequent 2,000 BNC/COCA word families. They were 123 word families with a dispersion of 0.6-0.69, and 178 word families with a dispersion of $0.7-0.79$. Their frequency ranged from 350 to 8,697 , and they represented items from a range of lower BNC/COCA frequency levels (Table 5.27). 
Table 5.27. Distribution of the 301 additional word families across the BNC/COCA word levels

\begin{tabular}{ll}
\hline COCA level & Number of word families \\
\hline $3^{\text {rd }} 1,000$ & 230 \\
$4^{\text {th }} 1,000$ & 46 \\
$5^{\text {th }} 1,000$ & 13 \\
$6^{\text {th }} 1,000$ & 6 \\
$7^{\text {th }} 1,000$ & 3 \\
$8^{\text {th }} 1,000$ & 1 \\
$9^{\text {th }} 1,000$ & 1 \\
Outside BNC/COCA & 1 \\
\hline Total & 301 \\
\hline
\end{tabular}

Most of these word families appeared in at least 20 out of 24 subjects (Table 5.28).

Noticeably, 51 word families occurred in all 24 subjects with frequency higher than 1,000 (e.g., function, method, element, solve, potential, solution, dimension, strategy, derive, component, reflect, random, evaluate, implement, conduct). Choosing the dispersion cut-off point of 0.8 would have excluded these high frequency and wide ranging items from the ASWL.

Table 5.28. Range of the 301 additional word families

\begin{tabular}{ll}
\hline Number of subjects & Number of word families \\
\hline 24 & 112 \\
23 & 56 \\
22 & 43 \\
21 & 27 \\
20 & 25 \\
19 & 16 \\
18 & 12 \\
17 & 4 \\
16 & 1 \\
15 & 4 \\
14 & 1 \\
\hline Total & 301 \\
\hline
\end{tabular}


Similarly, compared with 0.9 , the dispersion cut-off point of 0.6 included more academic words beyond the most frequent 2,000 BNC/COCA word families. If 0.9 were chosen, it would result in an ASWL, 99.38\% (319 out of 321 items) of which was from the BNC/COCA2000. In sum, the pilot list with the dispersion cut-off point of 0.6 better met the Word families outside general high-frequency word families characteristic of an ASWL than that with the dispersion cut-off point of 0.8 or 0.9 .

In sum, the comparison of ten pilot lists with different dispersion measures showed that the pilot list with the dispersion cut-off point of 0.6 best satisfied the four characteristics of the ASWL (see Section 5.6). Compared with 0.1 and 0.2, the dispersion cut-off point of 0.6 resulted in a word list that had fewer items but provided higher coverage than the most frequent 2,000 BNC/COCA word families. Compared with the lists with the dispersion cutoff points of $0.3,0.4,0.5$, or 0.7 , the list with a dispersion cut-off point of 0.6 distributed more evenly across the first academic spoken corpus. Compared with the lists with the dispersion cut-off points of 0.8 and 0.9 , the list with a dispersion cut-off point of 0.6 included more academic words beyond the general high-frequency words. The dispersion cut-off point of the ASWL was lower than that used to select Gardner and Davies's (2014) Academic Vocabulary List (AVL) words (0.8), which supports the findings of previous research that there is a clear-cut difference between the linguistic features of academic speech and academic writing (Biber, 2006; Biber, Conrad, Reppen, Byrd, \& Helt, 2002).

\subsection{Developing and validating the ASWL}

The previous sections have described the establishment of the range, frequency, and dispersion criteria of the ASWL. The next step was to select word families in the first academic spoken corpora that met these criteria and include them in the ASWL. These word families were then divided into four levels according to the BNC/COCA frequency levels (see Table 5.18 above).

The coverage of the ASWL and its levels in the two academic spoken corpora, the academic written corpus, and the non-academic spoken corpus was determined by running these corpora in turn through Nation et al.'s (2002) RANGE with the list and its levels serving as the base word lists. Similar steps were done to determine the coverage of the BNC/COCA2000 in the two corpora. The $1^{\text {st }}$ and $2^{\text {nd }} 1,000 \mathrm{BNC} / \mathrm{COCA}$ words were used as the baseword lists. 
To examine the overlap between academic spoken vocabulary with general high-frequency words and academic written vocabulary, the ASWL was compared with Nation's (2012) BNC/COCA2000 and two academic written word lists, Coxhead's (2000) AWL and Gardner and Davies's (2014) AVL. The BNC/COCA2000 was chosen to represent general high-frequency vocabulary because Studies 1 and 2 (Chapters 3 and 4) have suggested that it is the most suitable general high-frequency word list for L2 learners. Coxhead's (2000) AWL was chosen because it is the best-known academic written word list, and its validity has been confirmed by its consistently high coverage in numerous kinds of academic written texts (see Section 2.5.2.2 for more details about the AWL). Gardner and Davies's (2014) AVL was chosen because it is a recently created academic written word list that was validated in two studies (Durrant, 2016; Gardner \& Davies, 2014) (see Section 2.5.2.4 for more details about the AVL).

Another academic word list that was created recently is Browne et al.'s (n.d.) New Academic Word List (NAWL) (see Section 2.5.2.3 for more detail about the NAWL). This list was not used in the comparison for two reasons. First, the NAWL was created on top of Browne's (2014) New General Service List (NGSL) (see Section 2.8.1.4 for more details about the NGSL). As mentioned in Chapter 3 (Study 1), the NGSL provided lower coverage per item in 18 general English corpora than any of the four general highfrequency word lists including West's (1953) GSL. This limitation of the NGSL may have a negative impact on the value of the NAWL. Second, around 1.1\% of the corpus from which the NAWL was derived was spoken materials. Therefore, the NAWL may not be a truly academic written word list.

Coxhead's (2000) AWL was downloaded as part of the RANGE package that is available at Paul Nation's website. It contains 570 Level 6 word families. Gardner and Davies's (2014) AVL Level 6 word families was downloaded from Davies and Gardner's website, Academic Vocabulary Lists (http://www.academicvocabulary.info/). Although in its original format, the AVL was a lemma (Level 2 word family) list, Gardner and Davies also developed a Level 6 word family version of the AVL to make it possible to compare the AVL with other Level 6 word family lists. The AVL Level 6 word family list contains 1,991 items. However, unlike other Level 6 word family lists used in the present thesis, Gardner and Davies's (2014) AVL Level 6 word families distinguished between word 
classes. For example, view (n) and view (v) are considered as two separate word families. To be consistent, in the present study, eight repeated Level 6 word families were removed. As a result, the AVL Level 6 word family list used for this study had 1,983 word families. It should be noted that the AVL contains 12 proper nouns: Africa, Anglo, Asia, Assyria, Auspice, Darwinian, Dominican, Europe, Germany, Greece, Hellenic, and Tanzania. This approach is different from the approach taken by Coxhead (2000), Nation (2012), and the current researcher. Proper nouns were not counted in the selection of the AWL and the ASWL words because of the assumption that the learning burden of proper nouns is not as high as other common words (Coxhead, 2000; Nation \& Webb, 2011). However, in order not to change the nature of Gardner and Davies's (2014) AVL, these 12 proper nouns were kept in the list. Once the versions of Coxhead's (2000) AWL and Gardner and Davies's (2014) AVL were finalized, these lists were then compared with the ASWL to see the overlap between them.

\subsection{Determining the potential coverage that learners may reach by learning the ASWL}

This section describes how to calculate the potential coverage that learners with different levels (low, mid-low, mid, and high levels) may achieve if they learn the ASWL (see the definition of these groups in Table 4.11 above). The potential coverage for these learners is the sum of the coverage provided by two groups of words. The first group includes the word families that students may already know. They are items at the BNC/COCA levels that are relevant to students' existing vocabulary knowledge.

The second group is the coverage provided by the ASWL word families that students may not know. They are ASWL items that are outside the BNC/COCA words in the first group. Figure 5.1 demonstrates the components of the potential coverage for each group of learners. For example, low-level learners (i.e., those with the vocabulary level less than 1,000 word families) may gain the full coverage provided by the ASWL. For the mid-low group (i.e. those with the vocabulary level of the most frequent 1,000 word families), their potential coverage is the combination of the coverage provided by word families from the $1^{\text {st }} 1,000 \mathrm{BNC} / \mathrm{COCA}$ word families, and the ASWL words from Levels 2-4. 
Figure 5.1. Potential coverage reached by learners of different vocabulary levels with the aid of the ASWL

\section{Groups of learners Vocabulary level Sources of potential coverage}
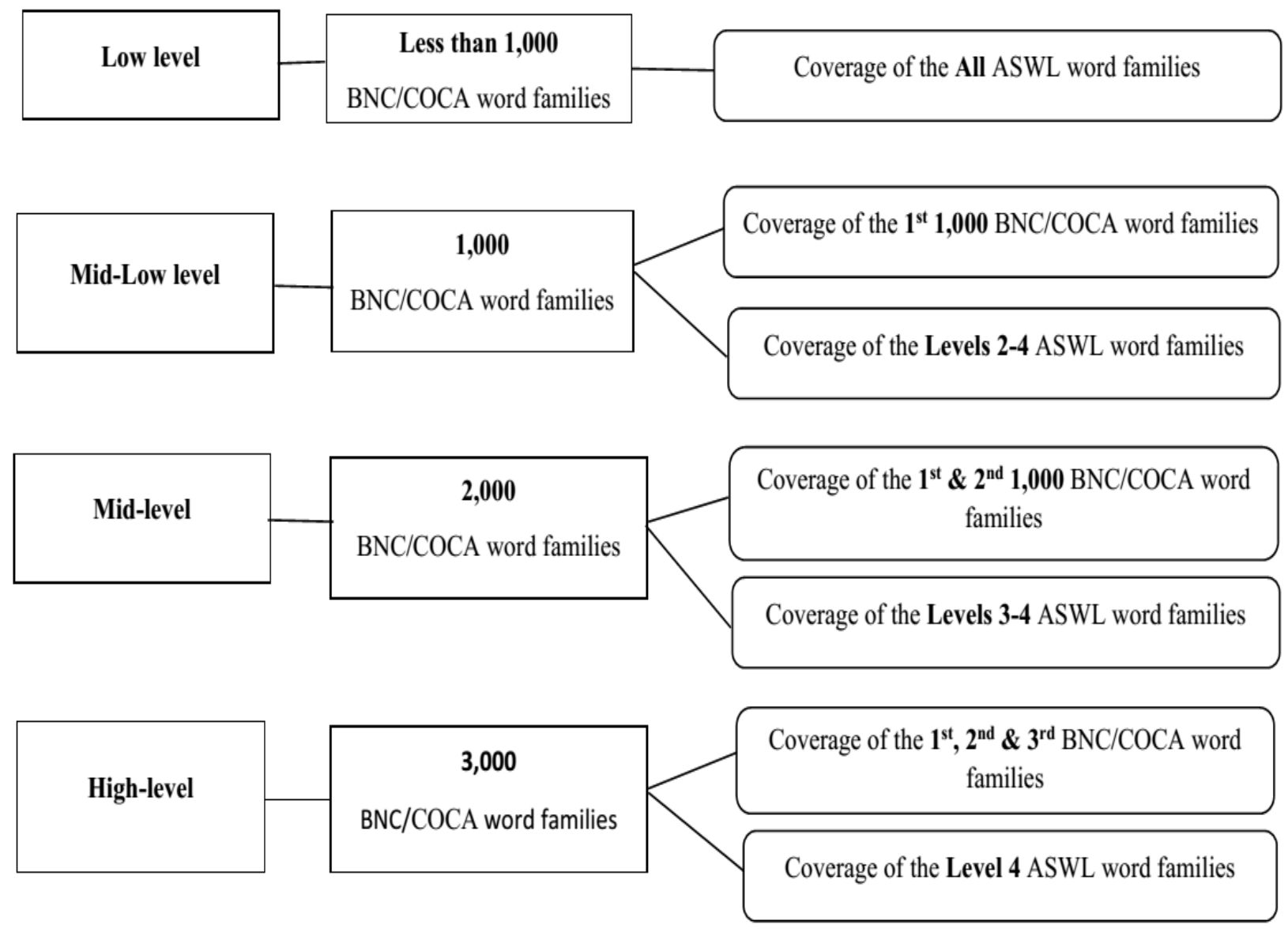

\subsection{Results}

So far in this chapter, the methodology of the present study has been described. In this section, we look at the findings of the study in response to the six research questions.

\subsubsection{The most frequent, wide ranging, and evenly distributed words in academic speech}

In answer to the first research question, 1,741 word families in the first academic spoken corpus satisfied the criteria for inclusion in the ASWL mentioned in Section 5.7 (see these word families in the Appendices 17-20). Table 5.29 presents the distribution of these word families across the BNC/COCA lists. Although most of them are among the most frequent 2,000 BNC/COCA words families, 454 word families are at lower BNC/COCA frequency 
levels ranging from the $3^{\text {rd }}$ to $10^{\text {th }} 1,000$-word levels, and one word family (so-called) is outside the $25 \mathrm{BNC} / \mathrm{COCA}$ lists. The 1,741 ASWL word families are divided into four levels. Levels 1 and 2 contain general high-frequency words from the most frequent 2,000 $\mathrm{BNC} / \mathrm{COCA}$ word families that also have high frequency, wide range, and even distribution in academic speech. Levels 3 and 4 are items that have high frequency in academic speech and beyond general high-frequency words.

Table 5.29. Lexical profile of the ASWL

\begin{tabular}{lllll}
\hline $\begin{array}{l}\text { ASWL } \\
\text { level }\end{array}$ & $\begin{array}{l}\text { BNC/COCA word } \\
\text { level }\end{array}$ & $\begin{array}{l}\text { Number } \\
\text { of word } \\
\text { families }\end{array}$ & $\begin{array}{l}\text { Coverage } \\
(\%)\end{array}$ & Examples \\
\hline Level 1 & $1^{\text {st }} 1,000$ & 830 & 81.62 & alright, know, stuff \\
\hline Level 2 & $2^{\text {nd }} 1,000$ & 456 & 5.23 & therefore, determine, approach \\
\hline Level 3 & $3^{\text {rd }} 1,000$ & 380 & 2.85 & achieve, significant, review \\
\hline Level 4 & $4^{\text {th }} 1,000$ & 49 & 0.28 & straightforward, differentiate \\
& $5^{\text {th }} 1,000$ & 13 & 0.08 & arbitrary, coefficient, analytic \\
& $6^{\text {th }} 1,000$ & 6 & 0.03 & radius, optimise, intuition \\
& $7^{\text {th }} 1,000$ & 3 & 0.01 & subtract, gamma, inverse \\
& $8^{\text {th }} 1,000$ & 1 & 0.004 & theorem \\
& $9^{\text {th }} 1,000$ & 1 & 0.01 & exponential \\
& $10^{\text {th }} 1,000$ & 1 & 0.01 & semester \\
& Outside BNC/COCA & 1 & 0.01 & so-called \\
\hline Total & & 1,741 & 90.13 & \\
\hline
\end{tabular}

The coverage of the ASWL levels in the first academic spoken corpus was $86.85 \%$ (Levels 1 and 2) and 3.28\% (Levels 3 and 4). This means, as a whole, the ASWL accounts for $90.13 \%$ of the first academic spoken corpus. This coverage is higher than the coverage of the most frequent 2,000 BNC/COCA word families (88.61\%). It should be noted that the former list has 259 fewer items than the latter list.

\subsubsection{Frequency of the ASWL words}

Table 5.30 provides an overview of the frequency of the ASWL word families. Although 350 was set as the minimum frequency of ASWL items, 57.04\% of the ASWL word families have a frequency higher than 1,000. It should be noted that word families at Levels 3 and 4 were not expected to have very high frequency because they are outside general high-frequency words. Yet, $28.57 \%$ of these words occur more than 1,000 times in the first 
academic spoken corpus, and the word family with the highest frequency in this group (function) occurs 8,697 times.

Table 5.30. Frequency of the ASWL levels in the $1^{\text {st }}$ academic spoken corpus

\begin{tabular}{lllll}
\hline \multirow{2}{*}{ Frequency } & \multicolumn{4}{l}{ Number of word families } \\
\cline { 2 - 5 }$>1000$ & Level 1 & Level 2 & Level 3 & Level 4 \\
$350-1000$ & 632 & 231 & 119 & 11 \\
\hline Total & 198 & 225 & 261 & 64 \\
Range of frequency & $351-791,050$ & $350-12,253$ & $350-8,697$ & $350-2,565$ \\
\hline
\end{tabular}

\subsubsection{Range of the ASWL words}

As presented in Table 5.31, although the minimum number of subjects in which an ASWL word family had to occur was 12 out of 24 subjects, all ASWL words appear in 14 subjects or more. A large number of ASWL items occur in a wide range of subject areas. $97.47 \%$ of the ASWL word families occur in at least 20 subject areas. In essence, $75.36 \%$ occur in all 24 subjects.

Table 5.31. Range of the ASWL words in the $1^{\text {st }}$ academic spoken corpus

\begin{tabular}{ll}
\hline Number of subjects & Number of word families \\
\hline 24 & 1,312 \\
23 & 185 \\
22 & 102 \\
21 & 55 \\
20 & 43 \\
19 & 20 \\
18 & 14 \\
17 & 4 \\
16 & 1 \\
15 & 4 \\
14 & 1 \\
\hline Total & 1,741 \\
\hline
\end{tabular}

Table 5.32 shows that, the ASWL items at the highest frequency levels tended to appear in the greatest range of subjects. Word families at Levels 1 and 2 have very wide range: from 18 to 24 subjects (Level 1) and 19-24 subjects (Level 2). Most of these items appeared in 
24 subjects. Word families at Levels 3 and 4 had narrower range than those at Levels 1-2.

Despite this fact, the range of Levels 3 and 4 word families is still wide: $15-24$ subjects

(Level 3) and 14-24 subjects (Level 4). The majority of ASWL word families occurred in 20 subjects with $56.58 \%$ of the word families at Level 3 and $26.67 \%$ word families at Level 4 appearing in 24 subjects.

Table 5.32. Range of the ASWL levels in the $1^{\text {st }}$ academic spoken corpus

\begin{tabular}{lllll}
\hline & \multicolumn{3}{l}{ Number of word families } & \\
\cline { 2 - 5 } Number of subjects & Level 1 & Level 2 & Level 3 & Level 4 \\
\hline 24 & 731 & 346 & 215 & 20 \\
23 & 65 & 42 & 63 & 15 \\
22 & 21 & 31 & 37 & 13 \\
21 & 6 & 21 & 25 & 3 \\
20 & 5 & 12 & 16 & 10 \\
19 & 0 & 4 & 11 & 5 \\
18 & 2 & 0 & 8 & 4 \\
17 & 0 & 0 & 2 & 2 \\
16 & 0 & 0 & 1 & 0 \\
15 & 0 & 0 & 2 & 2 \\
14 & 0 & 0 & 0 & 1 \\
\hline Total & 830 & 456 & 380 & 75 \\
\hline
\end{tabular}

\subsubsection{Dispersion of the ASWL words}

Table 5.33 shows that, although 0.6 was set as the dispersion cut-off point, $85.41 \%$ of the ASWL words have a dispersion of at least 0.7. Except for the case of dispersion from 0.9 to 1.0, as the degree of even distribution decreased, the number of lexical items decreased accordingly.

Table 5.33. Dispersion of the ASWL word in the $1^{\text {st }}$ academic spoken corpus

\begin{tabular}{ll}
\hline Dispersion & Number of word families \\
\hline $0.9-1.0$ & 321 \\
$0.8-0.9$ & 696 \\
$0.7-0.8$ & 470 \\
$0.6-0.7$ & 254 \\
\hline Total & 1,741 \\
\hline
\end{tabular}


A similar pattern was found when each level was examined. As shown in Table 5.34, there is a decrease in dispersion from Level 1 to Level 4. Most ASWL word families at Level 1 have a dispersion of 0.8 or above while most of those at Levels 2 and 3 have a dispersion from 0.7 to 0.9 . Word families at Level 4 have a lower dispersion. Four word families (straightforward, semester, fraction, domain) had a dispersion from 0.8 to 0.9 while most of them had a dispersion between 0.6 and 0.8 .

Table 5.34. Dispersion of each ASWL level in the $1^{\text {st }}$ academic spoken corpus

\begin{tabular}{lllll}
\hline \multirow{2}{*}{ Dispersion } & \multicolumn{4}{l}{ Number of word families } \\
\cline { 2 - 5 } & Level 1 & Level 2 & Level 3 & Level 4 \\
\hline $0.9-1.0$ & 295 & 24 & 2 & 0 \\
$0.8-0.9$ & 336 & 208 & 148 & 4 \\
$0.7-0.8$ & 137 & 155 & 145 & 33 \\
$0.6-0.7$ & 62 & 69 & 85 & 38 \\
\hline Total & 830 & 456 & 380 & 75 \\
\hline
\end{tabular}

\subsubsection{The ASWL sub-lists}

Following Dang and Webb (2016), each level of the ASWL was divided into a sub-list of function words and lists of lexical words. The lexical words were further divided into sublists of 50 word families or fewer according to the frequency of the word families in the first academic spoken corpus. The number of function words and lexical words in each sublist is presented in Table 5.35. The ASWL words in each sub-list can be seen in Appendices 17-20.

Table 5.35. Number of the ASWL sub-lists of function words and lexical words

\begin{tabular}{rrrrl}
\hline \multirow{2}{*}{ Level } & \multicolumn{3}{c}{ Number of items } & \multicolumn{2}{c}{ Sub-list of lexical words } \\
\cline { 2 - 5 } & $\begin{array}{l}\text { Function } \\
\text { words }\end{array}$ & $\begin{array}{l}\text { Lexical } \\
\text { words }\end{array}$ & Total & \\
\hline 1 & 141 & 689 & 830 & 13 sub-lists of 50 words \& 1 sub-list of 39 words \\
2 & 6 & 450 & 456 & 9 sub-lists of 50 words \\
3 & 0 & 380 & 380 & 7 sub-lists of 50 words \& 1 sub-list of 30 words \\
4 & 0 & 75 & 75 & 1 sub-list of 50 words and 1 sub-list of 25 words \\
\hline Total & 147 & 1,594 & 1,741 & \\
\hline
\end{tabular}

Although there are variations in the definitions of function and lexical words, in this study, Biber, Johansson, Leech, and Finegan's (1999) definitions were adopted. Lexical words are 
items conveying content meanings whereas function words are items expressing grammatical relationship. Words that can be either function words or lexical words (e.g., have, past) will be considered function words. The ASWL function words were determined based on Nation's (2016) list of function words. This list was originally from Biber et al. (1999) but then adapted for the RANGE program by Nation (2016).

Taken as a whole, the ASWL contains 1,741 word families. It covers $90.13 \%$ of the words in the first academic spoken corpus. ASWL words are high frequency, wide ranging, and evenly distributed items in academic speech. The coverage, frequency, range, and dispersion of the ASWL word families decrease from Level 1 to Level 4. ASWL words at Levels 1 and 2 are also general high-frequency words while those at Levels 3-4 are outside general high-frequency words. Because frequency is closely related to range and dispersion (Carroll, Davies, \& Richman, 1971; Nation, 2016), it is not surprising that Levels 1 and 2 word families have higher frequency, wider range, and higher dispersion than those at Levels 3 and 4. The remarkable point is that a considerable number of words at Levels 3 and 4 are still as frequent, wide ranging, and evenly distributed as those at Levels 1 and 2. Each level of the ASWL is further divided into sub-lists of function words and lexical words.

\subsubsection{Coverage of the ASWL in hard-pure, hard-applied, soft-pure, and soft-applied speech}

The second research question concerns coverage of the ASWL in academic speech in hardpure, hard-applied, soft-pure, and soft-applied subjects. Table 5.36 shows that, in all cases, the ASWL provides around $90 \%$ coverage of academic speech.

Table 5.36. Coverage of the ASWL in the sub-corpora of the two academic spoken corpora

\begin{tabular}{ll}
\hline Sub-corpus & Coverage $(\%)$ \\
\hline Hard applied & 91.07 \\
Soft applied & 90.92 \\
Hard pure & 89.46 \\
Soft pure & 89.00 \\
\hline
\end{tabular}


The coverage of the ASWL levels in each sub-corpus is presented in Table 5.37.

Irrespective of the ASWL levels, the coverage in the applied sub-corpus was slightly higher than the relevant pure sub-corpus. That is, the coverage in the hard-applied sub-corpus was higher than the hard-pure sub-corpus, and the coverage in the soft-applied sub-corpus was higher than the soft-pure sub-corpus. When the ASWL words at Levels 1 and 2, which are also general high-frequency words, were examined, these words always provided slightly higher coverage in the soft sub-corpus than in the relevant hard sub-corpus. In contrast, the ASWL words at Levels 3 and 4, which are not general high-frequency words, always covered a slightly larger proportion of the hard sub-corpus than the relevant soft subcorpus. Despite these differences, the coverage of the ASWL and its levels in the four subcorpora was generally similar.

Table 5.37. Coverage of the ASWL levels in each sub-corpus (\%)

\begin{tabular}{llllll}
\hline & Relevant BNC/COCA level & $\begin{array}{l}\text { Hard } \\
\text { pure }\end{array}$ & $\begin{array}{l}\text { Hard } \\
\text { applied }\end{array}$ & $\begin{array}{l}\text { Soft } \\
\text { pure }\end{array}$ & $\begin{array}{l}\text { Soft } \\
\text { applied }\end{array}$ \\
\hline Levels 1 \& 2 & $1^{\text {st }} \& 2^{\text {nd }} 1,000$ & 85.87 & 87.43 & 86.37 & 87.72 \\
Levels 3+4 & Beyond the $1^{\text {st }} \& 2^{\text {nd }} 1,000$ & 3.59 & 3.64 & 2.63 & 3.20 \\
\hline
\end{tabular}

\subsubsection{Coverage of the ASWL in academic speech, academic writing, and non- academic speech}

The third research question is related to the coverage of the ASWL in different discourse types. Table 5.38 presents the coverage of the ASWL in the three validating corpora.

Table 5.38. Coverage of the ASWL in the three validating corpora (\%)

\begin{tabular}{ll}
\hline Corpus & Coverage $(\%)$ \\
\hline 2nd academic spoken corpus & 89.59 \\
Non-academic corpus & 87.06 \\
Academic written corpus & 81.43 \\
\hline
\end{tabular}

The first row of the table shows that, as a whole, the ASWL covered around $90 \%$ of the words in the second academic spoken corpus. In fact, Levels 1 and 2 of the ASWL provided coverage of $86.34 \%$ while the coverage of Levels 3 and 4 was $3.25 \%$. These coverage figures are very similar to the coverage of the ASWL and its levels in the first 
academic spoken corpus. These findings indicate that the ASWL accurately captures high frequency, wide ranging, and even distributed word families in academic speech.

In contrast, the ASWL provided lower coverage of the academic written corpus than the first academic spoken corpus. This suggests that the list better represents spoken than written vocabulary. Similarly, its coverage in the non-academic spoken corpus was not as high as in the first academic spoken corpus. This demonstrates that the ASWL accurately represents academic rather than non-academic vocabulary. Taken together, these findings suggest that the ASWL truly reflect academic spoken vocabulary.

\subsubsection{General high-frequency words in academic spoken, non-academic spoken} English, and written English

The fourth research question is about the coverage of the BNC/COCA2000 in different kinds of discourse. The last column of Table 5.39 shows that, as a whole list, the BNC/COCA2000 provided the highest coverage in the non-academic spoken corpus. It is understandable because this list is a general high-frequency word list. Its coverage in the two academic spoken corpora was slightly lower.

Table 5.39. Coverage of the BNC/COCA2000 in the four corpora (\%)

\begin{tabular}{llll}
\hline & \multicolumn{3}{c}{ Coverage of the BNC/COCA2000 $(\%)$} \\
\cline { 2 - 4 } Corpus & $1^{\text {st }} 1,000$ & $2^{\text {nd }} 1,000$ & Total \\
\hline Non-academic spoken corpus & 84.38 & 4.34 & 88.72 \\
$1^{\text {st }}$ academic spoken corpus & 82.28 & 6.33 & 88.61 \\
$2^{\text {nd }}$ academic spoken corpus & 82.07 & 5.71 & 87.78 \\
Academic written corpus & 64.60 & 11.79 & 76.39 \\
\hline
\end{tabular}

In contrast, coverage of the BNC/COCA2000 in the academic written corpus was much lower. A similar pattern was seen when coverage of the $1^{\text {st }} 1,000 \mathrm{BNC} / \mathrm{COCA}$ words was examined (see the second column of the table). These words provided the highest coverage in the non-academic spoken corpus. Next came the two academic spoken corpora. The academic written corpus had the lowest coverage. However, when coverage of the $2^{\text {nd }} 1,000$ BNC/COCA words was determined, a reverse trend was witnessed (see the third column of the table). The academic written corpus ranked first. It is followed by the two academic spoken corpora. The non-academic spoken corpus ranked last. Together these findings indicate that, as a whole, general high-frequency words are more important in academic 
spoken English than in academic written English. However, the relative value of general high-frequency words changed according to the level. The most frequent 1,000 words are more significant in academic speech than in academic writing, but it is the other way round for the subsequent 1,000 words.

\subsubsection{General high-frequency words and academic written words in the ASWL}

The fifth research question is about the overlap between the ASWL and lists of general high-frequency words and academic written words. Table 5.40 presents the overlap between the ASWL and Coxhead's (2000) AWL, Nation's (2012) BNC/COCA2000, and Gardner and Davies's (2014) AVL. The first row of the table shows that the BNC/COCA2000 had the largest overlap with the ASWL. $73.87 \%$ of the ASWL $(1,286$ out of 1,741word families) appeared in the BNC/COCA2000. These items accounted for $86 \%$ $87 \%$ of academic speech.

Table 5.40. ASWL words in Nation's (2012) BNC/COCA2000, Coxhead's (2000) AWL, and Gardner and Davies's (2014) AVL

\begin{tabular}{|c|c|c|c|c|}
\hline \multirow[b]{2}{*}{ Word list } & \multirow{2}{*}{$\begin{array}{l}\text { Number } \\
\text { of words }\end{array}$} & \multicolumn{2}{|l|}{ Coverage $(\%)$} & \multirow[b]{2}{*}{ Examples } \\
\hline & & $\begin{array}{l}1^{\text {st }} \text { academic } \\
\text { spoken corpus }\end{array}$ & $\begin{array}{l}2^{\text {nd }} \text { academic } \\
\text { spoken corpus }\end{array}$ & \\
\hline BNC/COCA2000 & 1,286 & 86.85 & 86.34 & alright, though \\
\hline AVL & 763 & 22.45 & 22.38 & review, synthesis \\
\hline AWL & 368 & 3.66 & 3.52 & investigate, guarantee \\
\hline
\end{tabular}

The large overlap between the two lists may be the result of the nature of the ASWL and the BNC/COCA2000. Both lists were derived from spoken vocabulary. Moreover, the ASWL did not exclude general high-frequency words if they also had high frequency, wide range and even distribution in academic speech. However, this does not mean that the ASWL is a general high-frequency word list. A closer examination of the 1,286 overlapping word families between the ASWL and the BNC/COCA2000 reveals that, although they are general high frequency words, they reflect Csomay's (2006) construct of "on-line informational elaboration", a distinctive feature of academic speech. That is, the focus of the message is the information, but the information is transferred in real time production circumstance. 
The "on-line informational elaboration" feature is reflected in the ASWL by the fact that 218 ASWL items that also appear in the BNC/COCA2000 (e.g., seem, suppose, believe) are in Biber, Conrad, Reppen, Byrd, Helt, Clark, Cortes, Csomay, and Urzua's (2004) list of stances. Although, stances are, by definition, the means for writers or speaker to convey their personal feelings, attitudes, value judgments, or assessments (Biber et al., 1999). They are more likely to be used for function rather than the expression of epistemic or attitudinal meanings in academic speech (Biber, 2006). Academic speakers use them to guide listeners through steps of complex explanations, indicate the source of information, signal the extent to which information is known or doubtful, and convey personal attitudes about the course content. For example, in Excerpt [5.1], actually (an ASWL word family) indicates the speaker's certainty about the information while excellent (another ASWL word family) expresses his/her positive attitude towards the information.

\section{[5.1] the 2011 post mortem is actually an excellent write up by Steve Arcangeli}

(Electrical engineering, hard applied, $1^{\text {st }}$ academic spoken corpus)

These functions of stances reflect the on-line information elaboration feature in the sense that the main focus of the speech is to convey subject-related information; however, the shared contexts between speakers and listeners allow speakers to express their personal opinions about the information more openly.

Moreover, 30 items (e.g., okay, now, so) are in Fung and Carter's (2007) lists of discourse markers. Although there are different ways of classifying discourse markers, most items in Fung and Carter's (2007) list are single words. The ASWL words appearing in their list are much more common in academic speech than in academic writing (Biber, 2006; SimpsonVlach \& Ellis, 2010). They are the means for the speakers to signal the content and sequence of the ideas as well as indicating the boundary between relevant and irrelevant information (Eslami \& Eslami-Rasekh, 2007). The 30 ASWL words listed as discourse markers belong to one or more than one category in Fung and Carter's (2007) lists of discourse markers, including interpersonal category, referential category, structural category, and cognitive category. Interpersonal category (e.g., ok, alright, yes, well) consists of words marking shared knowledge, indicating attitudes, or showing responses. Referential category (e.g., because, however, and, so) includes conjunctions which express cause, contrast, coordination, disjunction, consequence, digression, and comparison. Words 
belong to the structural category (e.g., then, first, now, so) signal links and transitions between topics, indicate sequential relationships, and mark topic shifts. Lastly, words in the cognitive category (e.g., and, well) demonstrate the thinking process, reformulation, elaboration, or hesitations. For example, in the Excerpt [5.2] below, the speakers used alright and now to signal to the listeners about the close of a topic and the opening of a new one while okay was used as a means to check listeners' attention.

[5.2] Alright, anything else kind of on the administration angle that I could okay. Now I'm going to show you a movie that's going to make everything about pointers clear, okay?

(Computer science, hard applied, first academic spoken corpus)

Similar to stances, the presence of discourse markers in the ASWL demonstrates the on-line informational elaboration feature of academic speech. The density of the information and real time production require academic speakers to structure the information in a way that is easy for their listeners to process (Deroey \& Taverniers, 2011). Discourse markers are effective means for academic speakers to convey what is normally abstract information to listeners under time pressure.

So far in this section, we have found that the ASWL has a large number of shared items with the BNC/COCA2000. Despite this fact, these words reflect the on-line informational elaboration feature of academic speech. Let us now examine the overlap between the ASWL with the two academic written word lists. The second row of Table 5.40 above indicates that Gardner and Davies's (2014) AVL had the second largest overlap with the ASWL. Of the 1,741 words families, 763 items (43.77\%) were also AVL members. These words covered around $22 \%$ of academic speech. The last row of Table 5.40 demonstrates that Coxhead's (2000) AWL had the smallest overlap with the ASWL. Only 368 ASWL word families $(21.14 \%)$ occurred in the AWL. They provided less than $4 \%$ coverage of academic speech. It should be noted that the coverage of ASWL words overlapping with Coxhead's (2000) AWL in the two academic spoken corpora (3.66\% and 3.52\%) is slightly higher than the coverage of the ASWL words beyond the BNC/COCA2000 (3.28\%, $3.25 \%$ ). This is because $154 \mathrm{AWL}$ words are among the most frequent 2,000 BNC/COCA words. These words accounted for $1.91 \%$ and $1.82 \%$ of the $1^{\text {st }}$ and $2^{\text {nd }}$ academic spoken corpora, respectively. 
The difference in the principles of Gardner and Davies's (2014) AVL and Coxhead's (2000) AWL may be the reason why the former list had a larger overlap than the latter list. As mentioned in Section 2.5.2, Coxhead (2000) assumed that learners already know general high-frequency words, and therefore, did not include West's (1953) GSL words in her AWL. In contrast, Gardner and Davies (2014) did not assume that learners know general high-frequency words, and included in their AVL all items that have higher frequency and wider ranges in academic than in non-academic texts. This decision means that Gardner and Davies's (2014) AVL had a larger number of word families among the most frequent 2,000 BNC/COCA words (477) than Coxhead's (2000) AWL (154). This then results in the fact that the former list had larger overlap with the ASWL than the latter list.

In sum, the results show that the ASWL has considerable overlap with the list of general high-frequency words and some overlap with lists of academic written words. It was also found that, although a large number of ASWL items appeared in the general high-frequency word list, they may often have special function in academic speech.

\subsubsection{Potential coverage of academic speech that learners may reach if they learn the} ASWL

The last research question concerns the coverage that learners with different vocabulary levels may reach if they know the ASWL. Table 5.41 demonstrates the potential coverage that different groups of learners may gain with the aid of the ASWL. The second column shows the number of ASWL word families that are beyond learners' vocabulary levels. The next two columns show the potential coverage that learners may reach if they learn these ASWL words. Coverage provided by proper nouns (e.g., John, Berlin) and marginal words (e.g., $a h, h m m$ ) is presented in the last two rows. Previous research on vocabulary load of spoken English (e.g., Nation, 2006; Webb \& Paribakht, 2015; Webb \& Rodgers, 2009a, 2009b) assumed that proper nouns and marginal words have a minimal learning burden for learners, and therefore, added the coverage of these words to the potential coverage. The last two columns of Table 5.41 demonstrate the potential coverage including proper nouns and marginal words.

The first row of Table 5.41 shows the potential coverage for low level learners (i.e. those with the vocabulary level lower than the most frequent 1,000 BNC/COCA word families). These learners are not likely to know the ASWL due to their insufficient vocabulary knowledge. If they study all 1,741 ASWL word families, they may reach $90 \%$ coverage of 
the two academic spoken corpora. If proper nouns and marginal words are known, the potential coverage for these learners is $92.35 \%-92.60 \%$. These coverage figures are higher than those by the most frequent 2,000 BNC/COCA word families (90.54\%-91.08\%).

Table 5.41. Potential coverage gained by learners with the aid of the ASWL (\%)

\begin{tabular}{|c|c|c|c|c|c|c|}
\hline \multirow[t]{2}{*}{ Group } & \multirow{2}{*}{$\begin{array}{l}\text { Existing } \\
\text { vocabulary level } \\
\text { (BNC/COCA } \\
\text { word families) }\end{array}$} & \multirow{2}{*}{$\begin{array}{l}\text { Number of } \\
\text { ASWL word } \\
\text { families beyond } \\
\text { learners' level }\end{array}$} & \multicolumn{2}{|c|}{ Without PN \& MW } & \multicolumn{2}{|c|}{ With PN \& MW } \\
\hline & & & $\begin{array}{l}1 \mathrm{st} \\
\text { corpus }\end{array}$ & $\begin{array}{l}2 \text { nd } \\
\text { corpus }\end{array}$ & $\begin{array}{l}\text { st } \\
\text { corpus }\end{array}$ & $\begin{array}{l}\text { 2nd } \\
\text { corpus }\end{array}$ \\
\hline Low & Less than 1,000 & 1,741 & 90.13 & 89.59 & 92.60 & 92.35 \\
\hline Mid low & 1,000 & 911 & 90.79 & 90.12 & 93.26 & 92.88 \\
\hline Mid & 2,000 & 455 & 91.89 & 91.03 & 94.36 & 93.79 \\
\hline High & 3,000 & 75 & 93.33 & 92.24 & 95.80 & 95.0 \\
\hline \multicolumn{3}{|c|}{ Proper nouns (PN) } & 1.23 & 1.40 & & \\
\hline \multicolumn{3}{|c|}{ Marginal words (MW) } & 1.24 & 1.36 & & \\
\hline
\end{tabular}

The potential coverage for the mid-low level learners (i.e. those having mastered the most frequent 1,000 BNC/COCA word families) is presented in the second row of Table 5.41. With their existing knowledge, these learners only need to learn 911 ASWL word families. Yet, they may gain potential coverage of $90.12 \%-90.79 \%$. Including proper nouns and marginal words, the potential coverage that these learners may reach with the aid of the ASWL is just over 93\%. This is higher than the potential coverage they may gain if they study 1,000 words from the $2^{\text {nd }} 1,000$ BNC/COCA word level instead $(90.54 \%-91.08 \%)$. Interestingly, as shown in Table 5.42, learning the ASWL still allows learners from the low and mid-low groups to gain $92 \%-93 \%$ coverage of non-academic speech. These figures are fairly close to the coverage that learners may achieve if they study the BNC/COCA lists instead $(93.87 \%)$.

Table 5.42. Potential coverage of non-academic speech from different word lists for low and mid-low groups

\begin{tabular}{lll}
\hline $\begin{array}{l}\text { Group of learners } \\
\text { (vocabulary levels) }\end{array}$ & $\begin{array}{l}\text { Number of ASWL word families } \\
\text { beyond learners' level }\end{array}$ & $\begin{array}{l}\text { Potential } \\
\text { coverage (\%) }\end{array}$ \\
\hline Less than 1,000 word families & 1,741 & 92.21 \\
1,000 word families & 911 & 93.45 \\
\hline
\end{tabular}


Learners with higher vocabulary levels may be able to reach even higher potential coverage of academic speech by acquiring a smaller number of words. The third row of Table 5.41 shows that, mid-level learners (i.e., those with the vocabulary level of the most frequent 2,000 BNC/COCA word families) only have to learn 455 ASWL words, but can gain the potential coverage of $91.03 \%-91.89 \%$ (without proper nouns and marginal words) and $94 \%$ (with proper nouns and marginal words). For the high-level learners (i.e. those with the vocabulary level of the most frequent 3,000 BNC/COCA word families) (see the fourth column of Table 5.41), knowledge of 75 ASWL word families that are beyond their vocabulary levels may enable them to achieve coverage of $92.24 \%-93.33 \%$. Plus proper nouns and marginal words, the potential coverage is $95 \%-95.80 \%$. Taken as a whole, depending on learners' existing vocabulary level, the potential coverage including proper nouns and marginal words ranges from $92 \%$ to $96 \%$.

\subsection{Summary of main findings}

The most important finding of this study is related to the development of the ASWL. There are 1,741 word families which are the most frequent, wide ranging and evenly distributed in academic speech. They are included in the ASWL, which is divided into four levels according to Nation's (2012) BNC/COCA lists. Levels 1 and 2 represent general highfrequency words that also have high frequency, wide range, and even distribution in academic speech. Levels 3 and 4 are words that are not general high-frequency words but are frequent, wide ranging, and evenly distributed items in academic speech. The ASWL consistently provided around $90 \%$ coverage of the academic spoken corpus from which it was developed and an independent academic spoken corpus. This coverage was higher than the coverage of the list in the academic written corpus and the non-academic spoken corpus. This finding suggests that the ASWL is a truly academic spoken word list.

In chapter 6, this finding will be examined more closely from different perspectives. It will be used to highlight the value of general high-frequency words in comprehending academic spoken English (see Section 6.2.1). Moreover, it is mentioned in the discussion about the most suitable size of a general high-frequency word list for L2 learners (see Section 6.2.3). Also, it is used to demonstrate how word lists can be made adaptable to learners' proficiency (see Section 6.3.1) and academic disciplines (see Section 6.3.2). 
The present study also has other important findings that provide further insight for the discussion in Chapter 6. The first finding is related to the coverage of the ASWL in different disciplinary groups. The ASWL covered around $90 \%$ of the words in academic speech in hard-pure, hard-applied, soft-pure, and soft-applied subjects. However, it provided slightly higher coverage in the applied sub-corpora than in the pure sub-corpora. Moreover, the ASWL words at Levels 1 and 2 that are also general high-frequency words provided slightly higher coverage in the soft sub-corpora than in the hard sub-corpora. However, it was the other way around at Levels 3 and 4. In Chapter 6, this finding will be drawn together with the findings from other studies to discuss how word lists can be made adaptable to learners' academic disciplines (Section 6.3.2).

The second finding is related to general high-frequency words. The ASWL had a larger number of shared items with the list of general high-frequency words (BNC/COCA2000) than with lists of academic written words (AWL and AVL). The BNC/COCA2000, especially the most frequent 1,000 BNC/COCA words, provided much higher coverage in academic speech than academic writing. In Chapter 6, this finding will be discussed in detail to highlight the value of general high-frequency words in academic spoken English (see Section 6.2.1).

Last but not least, the present study reveals that, depending on their existing levels, learners can achieve 92\%-96\% coverage of academic speech with the aid of the ASWL. This finding will be used in Section 6.3.1 to demonstrate how word lists can be made adaptable to learners' proficiency levels.

\subsection{Rationale for the next chapter}

The first two studies of this thesis examined general high-frequency words from the perspectives of corpus linguistics, teachers, and learners. The third study explored the nature of vocabulary in academic speech in comparison with that of academic writing and non-academic speech. In the next chapter, the findings of these three studies will be discussed together. 


\section{Chapter 6 - Discussion}

\subsection{Introduction}

Chapters 3 to 5 have presented the answers to the research questions as well as interesting findings that emerged from each study. In this chapter, these findings are discussed in depth in three themes: (1) general high-frequency words, (2) word list construction, and (3) teachers' familiarity with learners' characteristics in second language (L2) vocabulary learning and teaching. The first two themes lie at the heart of vocabulary studies and run through all three studies of the present thesis. The last theme is an underexplored but potential area of vocabulary research. Let us now look at each of them in detail.

\subsection{What is the importance of general high-frequency words in $\mathrm{L} 2$ vocabulary learning?}

General high-frequency vocabulary is one core concept in vocabulary research. The importance of this kind of vocabulary is clearly demonstrated in all three studies of this thesis. This section (1) highlights the great value of general high-frequency words in academic spoken English, (2) suggests the most suitable general high-frequency word list for L2 learners, and (3) raises the question of the appropriate size of a general highfrequency word list for L2 learners.

\subsubsection{What is the value of general high-frequency words in academic spoken English?} Nation (2013) suggests that general high-frequency words are so important that they deserve considerable attention from teachers so that learners will fully master these words. The thesis reinforces this idea by revealing that knowledge of general high-frequency words helps learners to recognize a large proportion of words in academic spoken English, and enhances their comprehension of this discourse type.

To begin with, the value of general high-frequency words in academic speech can be seen clearly from the structure of the Academic Spoken Word List (ASWL), which represents the most frequent, wide ranging, and evenly distributed words in academic speech. More than $70 \%$ of the ASWL words appear at the $1^{\text {st }}$ and $2^{\text {nd }} 1,000 \mathrm{BNC} / \mathrm{COCA}$ frequency levels. In essence, about $48 \%$ of the ASWL words are the most frequent 1,000 BNC/COCA words. 
The ASWL has a larger proportion of shared items with the BNC/COCA2000 (73.87\%) than with the two academic written word lists, Coxhead's (2000) Academic Word List (AWL) (21.14\%) and Gardner and Davies's (2014) Academic Vocabulary List (AVL) $(43.77 \%)$.

The importance of general high-frequency words in academic spoken English is also apparent from the relative value of these words in academic speech compared with academic writing. The most frequent 2,000 BNC/COCA word families comprised around $88 \%-89 \%$ of the words in the two academic spoken corpora but only around $76 \%$ of the words in the academic written corpus. Remarkably, the coverage of the most frequent 1,000 BNC/COCA words in the two academic spoken corpora (around 82\%) is much higher than in the academic written corpus (less than 65\%). It is even higher than the coverage provided by the most frequent 2,000 BNC/COCA words in the academic written corpus.

Earlier research also reported a similar pattern. West's (1953) General Service List (GSL) provided from $85 \%$ to $87 \%$ coverage of academic spoken English (Dang \& Webb, 2014; Thompson, 2006), which is higher than its coverage of academic written English (from 65\% to 86\%) (Coxhead, 2000; Konstantakis, 2007; Valipouri \& Nassaji, 2013). Likewise, the most frequent 1,000 GSL words covered a larger proportion (approximately 82\%) of academic speech (Dang \& Webb, 2014) than academic writing (around 71\%) (Coxhead, 2000). Similarly, Nation's (2006) most frequent 2,000 BNC word families (BNC2000) accounted for higher coverage in academic speech (about 90\%) (Dang \& Webb, 2014) than in academic writing (81\% to 90\%) (Coxhead, Stevens, \& Tinkle, 2010; Nation, 2004). This thesis, however, advances the field of vocabulary in two ways. First, while West's (1953) GSL or Nation's (2004) BNC2000 was used to represent general high-frequency words in previous research, this study used Nation's (2012) most frequent 2,000 word families (BNC/COCA2000). As discussed later in Section 6.2.2, the BNC/COCA2000 is the most suitable general high-frequency word list for L2 learners from the perspectives of corpus linguistics, teachers, and learners. Second, the academic spoken corpora on which the analysis of this thesis was based are much larger, more representative, and balanced than the academic spoken corpora used in previous studies. Given these two strengths, this thesis provides a more thorough picture about the performance of general high-frequency words in academic spoken English than earlier research. In brief, it demonstrates that 
general high-frequency words, especially the most frequent 1,000 words, are more common in academic spoken language than in academic written language.

There are two possible reasons why general high-frequency words occur more often in academic spoken English than in academic written English. The first reason is the difference between the nature of written and spoken discourse (Biber, 2006). Academic writers have extensive time to plan, write, and edit their texts. In contrast, although academic speakers may have time to plan the content of their talk (e.g., lectures, tutorials), they still have to produce the language in real time circumstances. Therefore, while academic writers can select and make use of a wide range of low-frequency words to express their ideas more precisely, academic speakers are more likely to rely on general high-frequency words.

The second reason is the difference in the functions of academic writing and academic speaking. Although the primary purpose of both registers is subject-related information (Csomay, 2006; Deroey \& Taverniers, 2011), academic speech also has another important function, classroom management (Biber, 2006; Deroey \& Taverniers, 2011). This is the function in which the speakers manage organizational matters to ensure that listeners have necessary information about the courses and learning tasks (Deroey \& Taverniers, 2011). This function can be illustrated in the following excerpts which were taken from the two academic spoken corpora in the present study.

\section{[6.1] I'm planning on posting the exam at seven p.m. tonight}

(Computer Science, Hard-applied, $1^{\text {st }}$ academic spoken corpus)

[6.2] I hope you can play an active role in learning rather than sit down and wait for the material I give you you have to go to the library and search for the reference books

(History, Soft-pure, $2^{\text {nd }}$ academic spoken corpus)

Excerpt [6.1] is the lecturer's announcement about the exam information while Excerpt [6.2] is the lecturer's expectation about the students' participation in the course. It can be seen from these excerpts that classroom management is closer to general conversation than academic writing. Therefore, it is understandable why the vocabulary in academic speech is made up of a large proportion of general high-frequency words. 
Given the close relationship between vocabulary knowledge and comprehension, to enhance their comprehension of academic spoken English, it is extremely important for L2 learners to master general high-frequency words. One question that arises then is which general high-frequency word list should learners focus on? The next section discusses this issue in detail.

\subsubsection{What is the most suitable general high-frequency word list for L2 learners?}

While there are several general high-frequency word lists, it is unclear from previous research which list is the best for L2 learners. By examining the issue from multiple angles (corpus linguistics, teachers, and learners), Studies 1 and 2 indicate that Nation's (2012) $\mathrm{BNC} / \mathrm{COCA} 2000$ is the most suitable general high-frequency word list for L2 learners.

First, the BNC/COCA2000 provided higher coverage than West's (1953) GSL, Nation's (2006) BNC2000, and Brezina and Gablasova's (2015) New General Service List (NewGSL) in a wide range of corpora which represent different spoken and written discourse types and varieties of English. The excellent performance of the BNC/COCA2000 over the other lists may be because of the corpus used to create it. The ideal corpus to create a general high-frequency word list should consist of an equal proportion of spoken and written texts (Nation \& Waring, 1997; Nation, 2004). Additionally, texts from different sources and different varieties of English should be included to reflect L2 learning purposes (Nation, 2004). The corpora from which the GSL, the BNC2000, and the New-GSL were derived do not meet this guideline and consist of mainly written texts. The GSL was derived from a purely written corpus, while only $10 \%$ of the corpus on which the BNC2000 was based were spoken materials. Similarly, only one out of three corpora from which the New-GSL was developed consisted of spoken materials, and these materials made up only $10 \%$ of this corpus. In contrast, the corpus from which the BNC/COCA2000 was developed had a better balance of spoken $(60 \%)$ and written (40\%) samples. Moreover, these materials came from different sources (e.g., telephone conversation, movies, TV programs, written texts for young children and fiction) and represented different varieties of English (BritishEnglish, American-English, and New Zealand-English). As a result, the corpus used to create the BNC/COCA2000 may provide a more accurate picture of general high-frequency vocabulary than the corpora used to develop the other two lists. 
Second, compared with Brezina and Gablasova's (2015) New-GSL, the BNC/COCA2000 consistently had more words known by learners and perceived as being useful by teachers. It is understandable when considering the principles under which the two lists were developed. The New-GSL was created with a purely quantitative approach; that is, using frequency, dispersion, and distribution across language corpora as criteria in word selection. In contrast, apart from the quantitative criteria on which it was based, the development of the BNC/COCA2000 involved adding to the list the lexical items that did not meet the range, frequency, and dispersion criteria but may be suitable for L2 learning and teaching purposes. A word list that is solely based on the information from corpora may not include items that are low frequency in corpora but are useful for L2 learners and teachers (Nation, 2016). As shown in Section 6.3.3 below, information from corpora should be supplemented with information from teachers and learners to achieve better outcomes when introducing corpus-based word lists for L2 classrooms. The greater focus on L2 learning explained why the BNC/COCA2000 had better performance than the New GSL when teachers and learners were involved in the word list validation.

In brief, by looking at the value of the four general high-frequency word lists from different perspectives, this thesis provides sound evidence that Nation's (2012) BNC/COCA2000 is the best general high-frequency word list for L2 learners. This finding is meaningful given that no studies have compared a large number of general high-frequency word lists using multiple approaches to data analysis before.

\subsubsection{How many items should be in a general high-frequency word list?}

This thesis also raises the question about the value of making smaller lists of general highfrequency words to meet short-term learning goals. Although there are no fixed upper limits to the number of general high-frequency words (Nation, 2013; Schmitt, 2010), all existing general high-frequency word lists have around 2,000 to 3,000 items (see Section 2.8.1). The 2,000 figure is influenced by the size of West's (1953) GSL, which has around that number (Nation, 2016; Schmitt, 2010). It was further reinforced as the cut-off point of general highfrequency words by Nation and Hwang (1995) who examined the boundary between general high-frequency vocabulary and specialized vocabulary, and Nation (2001) who determined the cut-off point of general high-frequency words from different perspectives (coverage, cost/benefit analysis, overlap of different lists, total number of words, and meaning-use). The 3,000 figure is promoted by Schmitt and Schmitt (2014) who 
investigated this issue from the perspectives of frequency, coverage, acquisition, and use. The 3,000-word figure is also supported by Sorell's (2013) study that found that lists of the most frequent 3,000 words were sometimes superior to shorter lists.

The suitability of a general high-frequency word list that consists of 2,000 or more items, however, has been questioned by Engels (1968) who examined the distribution of West's (1953) GSL in ten 1,000 running word texts. Although Engels (1968) confirmed that the $1^{\text {st }}$ 1,000 high-frequency words were very good items in terms of frequency and range, he also questioned the necessity of words beyond the $1^{\text {st }} 1,000$ word level due to their low frequency and range. Other researchers (Dang \& Webb, 2016; Todd, 2017; Ward, 2009) shared the same idea with Engels (1968) by pointing out that a 2,000 or 3,000 word list may be too long for practical use. This thesis provides strong support for this argument in two ways.

First, as can be inferred in Section 6.2.1, no matter which word list is used to represent general high-frequency words, the majority of coverage provided by general highfrequency words in academic speech come from the most frequent 1,000 words. It then highlights the importance of the $1^{\text {st }} 1,000$ words over the $2^{\text {nd }} 1,000$ words in academic spoken English. In fact, the huge gap in the coverage provided by the $1^{\text {st }}$ and $2^{\text {nd }} 1,000$ words of general vocabulary is also evident in non-academic English. As shown in Study 1, the most frequent 1,000 headwords in all four general high-frequency word lists provided a much larger coverage (51.17\%-69.51\%) than the subsequent 996 headwords $(0.67 \%$ $4.72 \%$ ) in different non-academic spoken and written texts. Earlier corpus-driven vocabulary research also found a huge gap between the amount of coverage provided by the $1^{\text {st }}$ and $2^{\text {nd }} 1,000$ word levels in different discourse types (e.g., Nation, 2006; Webb \& Rodgers, 2009a, 2009b). These findings support Engels's (1968) claim that the most frequent 1,000 words are more stable than the subsequent 1,000 words. However, it is important to note that Engels's (1968) claim was based on the analysis of the range and frequency of West's (1953) GSL words in 10 non-academic written texts of 1,000 running words. In contrast, the conclusion made in this thesis is grounded in the analysis of a more updated general high-frequency word list (BNC/COCA2000) in academic spoken texts as well as a wide range of non-academic spoken and written texts with much larger sizes and greater degree of varieties. As a result, it provides more solid evidence of the greater 
importance of the $1^{\text {st }} 1,000$ words than the $2^{\text {nd }} 1,000$ words in academic spoken English in particular, and in any discourse types in general.

Second, Study 2 of this thesis found that a considerable number of L2 learners lack knowledge of general high-frequency words. As a whole, the learner participants had not mastered any level of Schmitt, Schmitt, and Clapham's (2001) Vocabulary Levels Test (VLT) after an average of nine years studying English. Nearly 65\% of them had not mastered the 2,000-word level, and scores suggested that, more than 50\% of the participants were unlikely to have mastered the $1^{\text {st }} 1,000$-word level. It should be noted that Study 2 looked at receptive knowledge of form and meaning, a basic aspect of vocabulary knowledge (Schmitt, 2010; Webb \& Chang, 2012). Learning and using a word receptively is much easier than learning and using it productively (Nation, 2013). Thus, it is reasonable to expect that these learners' productive levels are even lower. The results are consistent with those of previous research with learners in different English as a Foreign Language (EFL) contexts such as India (Barnard, 1961), Indonesia (Nurweni \& Read, 1999; Quinn, 1968), Denmark (Henriksen \& Danelund, 2015), Taiwan (Webb \& Chang, 2012), Israel (Laufer, 1998), and Vietnam (Nguyen \& Webb, 2016). These studies reported that a number of EFL learners failed to master general high-frequency words, and even the most frequent 1,000 words after a long period of formal instruction.

There are two possible reasons for this alarming picture of L2 learners' vocabulary levels. One reason may be the lack of a systematic focus on general high-frequency vocabulary in L2 learning programs. This echoes the argument that institutional language learning programs should pay more attention to this kind of vocabulary so that class time will be well spent helping learners master the words that are crucial for their language development (Dang \& Webb, 2016; Nation, 2016; Webb \& Chang, 2012). Another reason may be because of the size of existing general high-frequency word lists. A 2,000 or 3,000-item list may be too daunting a goal for L2 learners, especially beginners, considering their slow rate of vocabulary development and limited class time. It also creates challenges for individual teachers and even institutions to incorporate into a course and into multi-year language learning programs. Moreover, it may distract learners' attention from learning the $1^{\text {st }} 1,000$ words, which have much greater value in terms of lexical coverage than the next 1,000 words. 
Together, the relative value of the $1^{\text {st }}$ and $2^{\text {nd }} 1,000$ general high-frequency words and L2 learners' insufficient knowledge of these words suggest that it may be more effective and practical to break a general high-frequency word list down into frequency-based sub-lists with manageable sizes. This makes it easier for teachers to use these lists in planning shortterm learning goals. Also, it better scaffolds learners' vocabulary development. It ensures that learners will study the most frequent items first, and knowledge of these items may then support learners' acquisition of items in lower frequency sub-lists. The benefit of sublists with attainable learning sizes is evident in the popularity of Coxhead's (2000) AWL which contains sub-lists of 60 items. In terms of general high-frequency vocabulary, perhaps Dang and Webb's (2016) Essential Word List is the first attempt to make a corpusbased general high-frequency word list more practicable. This 800 lemma word list is divided into one list of 176 function words and 13 sub-lists of lexical words. Each sub-list of lexical words consists of 50 items. However, it should be noted that Dang and Webb's (2016) list particularly targets L2 beginners. Given that Nation's (2012) BNC/COCA2000 is the most suitable general high-frequency word list for L2 learners in general, a useful direction is to divide this list into sub-lists with a more attainable size so that the BNC/COCA2000 can better assist learners and teachers to set short-term learning goals.

In sum, this section has discussed the findings related to general high-frequency words. It emphasizes the importance of these words for L2 learners' comprehension of academic spoken English. Also, it indicates that Nation's (2012) BNC/COCA2000 is the most suitable general high-frequency word list for L2 learners. Considering the insufficient knowledge of these words possessed by a reasonable proportion of L2 learners, this thesis calls for more attention to these words and suggests that existing general high-frequency word lists should be broken down into sub-lists with manageable sizes to better support L2 vocabulary learning and teaching.

\subsection{How can word lists better support $L 2$ vocabulary development?}

Apart from general high-frequency words, another thread that runs through all three studies

of this thesis is word list construction. Given the dramatic increase in the number of available general high-frequency word lists and specialized word lists, researchers (e.g., Nation, 2016; Schmitt, 2016) have recently pointed out that, rather than uncritically 
replicating previous studies, it is important for list makers to consider the characteristics of their target users in word list development. This idea is clearly demonstrated in the findings of the current thesis. The previous section has touched on this issue to some extent from the perspective of the appropriate length of a general high-frequency word list for L2 learners. In this section, we will look more closely at other factors related to word list construction emerging from the results of this thesis. They are learner proficiency, academic majors, and the relationship between the information from corpora, teachers, and learners. Each issue is discussed in turn below.

\subsubsection{How can word lists be adaptable to learners' proficiency?}

Most existing academic word lists (Browne, Culligan, \& Phillips, n.d.; Coxhead, 2000; Nesi, 2002; Xue \& Nation, 1984) were created with the assumption that learners have learned and mastered the most frequent 2,000 or even 3,000 words of general vocabulary before studying items from their lists (see Section 2.5). Given the importance of these words, it is sensible for L2 learners who plan to study English for academic purposes to start their vocabulary learning after learning general high-frequency words. However, as discussed in Section 6.2.3 above, mastering general high-frequency words before learning items from academic word lists may be too demanding a goal for a proportion of L2 learners in certain contexts. Therefore, it is beneficial to develop an academic word list that is adaptable to learners' varying proficiency levels. The ASWL is an example of how an academic word list can better match learners' proficiency levels. This list is divided into four levels according to Nation's (2012) BNC/COCA lists. Level 1, 2, and 3 represent the ASWL words at the $1^{\text {st }}, 2^{\text {nd }}$, and $3^{\text {rd }} 1,000 \mathrm{BNC} / \mathrm{COCA}$ frequency levels, respectively. The ASWL words at Level 4 are those outside the most frequent 3,000 BNC/COCA words. These levels allow learners to focus their attention on the ASWL words that are relevant to their existing vocabulary levels. This enables the ASWL to better scaffold the vocabulary development of learners with different proficiency levels. Let us look at the benefit that the ASWL offers to each group of learners in detail.

\subsubsection{Mid and high-level proficiency learners}

The present thesis defines mid-level learners as those with the vocabulary levels of the most frequent 2,000 word families while high-level learners are those with the vocabulary level of 3,000 word families or more (see Table 4.11). As shown in Study 3, these learners may achieve around $95 \%$ coverage of academic speech by learning a small number of items 
from the ASWL: 455 word families (mid-level learners) or 75 word families (high-level learners). Knowing at least $95 \%$ of the words in spoken discourse is important for learners (Schmitt, Cobb, Horst, \& Schmitt, 2015). It allows them to obtain a high and stable degree of listening comprehension (van Zeeland \& Schmitt, 2013). Previous research (Dang \& Webb, 2014; Webb \& Paribakht, 2015) found that a vocabulary size of 4,000 word families is needed to reach $95 \%$ coverage of academic speech. This means that mid-level learners may need to learn a further of 2,000 word families from the $3^{\text {rd }}$ and $4^{\text {th }} 1,000$ word levels. Meanwhile, high-level learners may need to study an extra 1,000 word families from the $4^{\text {th }}$ 1,000 word level. Studying the ASWL word families that are beyond their levels gives these learners a better return. They need to learn a much smaller number of items but are still able to achieve $95 \%$ coverage of academic spoken discourse.

\subsubsection{Low and mid-low level proficiency learners}

In this thesis, low-level learners are those with the vocabulary level of less than 1,000 word families while mid-low level learners are those with the vocabulary level of 1,000 word families. Ideally these learners would study the most frequent 2,000 or even 3,000 BNC/COCA word families and then move to the relevant ASWL levels so that they can reach $95 \%$ of academic spoken discourse. However, this may be too demanding a goal for them, especially those studying in EFL contexts. Given that L2 learners may acquire an average of 400 word families a year (Webb \& Chang, 2012), the low and mid-low level learners may need about six years to acquire the most frequent 2,000 word families plus 455 extra ASWL word families, or eight years to acquire the most frequent 3,000 word families plus 75 extra ASWL word families. As mentioned in Section 6.2.3, research with L2 learners in different EFL contexts suggests that some learners may have even slower vocabulary growth rates, which means it may often be challenging for EFL learners to master 2,455 or 3,075 word families by the time they start their study in English-medium universities. Going straight to the ASWL or learning the ASWL from Level 2 can help with this dilemma to some degree. It focuses the attention of low and mid-low learners on the most important words in academic speech, meanwhile, allowing them to make up for their insufficient knowledge of general high-frequency words.

Learning the ASWL words can help low and mid-low level learners to achieve 90\%-91\% and $92 \%-93 \%$ coverage of academic speech without and with knowledge of proper nouns 
and marginal words, respectively. These figures are meaningful in three ways. First, these learners have to study a much smaller number of items, but can achieve higher coverage of academic speech than learning words from the subsequent levels of general vocabulary. Second, although comprehension may not be as easy as with $95 \%$ coverage, $90 \%-93 \%$ coverage may still allow L2 learners to achieve basic comprehension of academic speech. Van Zeeland and Schmitt (2013) found no significant difference in L2 listening comprehension between the $90 \%$ and $95 \%$ coverage figures. Both coverage figures led to $70 \%$ listening comprehension. Moreover, if $70 \%$ comprehension was considered as an indicator of adequate comprehension, both figures resulted in $75 \%$ of the participants achieving this result. Even with a stricter criterion of adequate comprehension (80\%), more than half of the participants met this requirement at both coverage levels. Furthermore, in real life academic speech, students receive support to facilitate their listening comprehension such as pre-lecture reading materials, visual aids, or interaction with their lectures, tutors, and peers, which may allow L2 learners to compensate for their inadequate vocabulary knowledge and enhance their listening of academic speech (Flowerdew \& Miller, 1992; MacDonald, Badger, \& White, 2000; Mulligan \& Kirkpatrick, 2000). Third, going straight to the ASWL or learning the ASWL from Level 2 may also enable low and mid-low level learners to reach 92\%-93\% of general spoken English, which may allow them to understand this important discourse type. In brief, the ASWL is a good shortcut for these learners to achieve basic comprehension of academic speech while still allowing them to enhance their knowledge of general high-frequency words.

In brief, this section has demonstrated that, if learner proficiency is taken into account in word list construction, it will result in more efficient vocabulary learning. In the next section, we will examine word list construction from the perspective of learners' academic disciplines.

\subsubsection{How can word lists be adaptable to learners' academic disciplines?}

The nature of vocabulary in academic speech across different disciplines revealed in this thesis provides further insight into the debate over the value of universal academic word lists versus discipline-specific word lists. One view suggests that there is a core vocabulary across multiple academic disciplines. Therefore, it supports the development of universal academic word lists for L2 learners irrespective of their academic disciplines (e.g., Coxhead, 2000; Gardner \& Davies, 2014; Xue \& Nation, 1984). A second view questions 
the existence of a core academic vocabulary from different academic disciplines and argues that frequency, range, meanings, functions, and collocations of a certain word change across disciplines due to the variations in the practice and discourse of disciplines (Durrant, 2014; Hyland \& Tse, 2007). Hence, it promotes the idea of developing discipline-specific word lists in a particular subject area such as engineering (Mudraya, 2006; Todd, 2017; Ward, 1999, 2009), medicine (Hsu, 2013; Lei \& Liu, 2016), agriculture (Martínez et al., 2009), chemistry (Valipouri \& Nassaji, 2013), environmental science (Liu \& Han, 2015), or nursing (Yang, 2015). The differences and similarities between the vocabulary in different disciplines found in Study 3 suggests that, depending on the academic disciplines of the learners in a certain English for Academic Purposes (EAP) program, one kind of word list may be more suitable than the other.

\subsubsection{What are the differences between vocabulary in different disciplines?}

Study 3 reveals that the vocabulary in academic speech in different disciplines varies to some extent. This variation can be seen in both the pure/applied dimension and the hard/soft dimension. As mentioned in Section 5.3.1.1 above, the pure/applied dimension is related to the application of practical problems while the hard/soft dimension associates with the existence of a paradigm. Some examples of academic subjects within these disciplines are Mathematics and Physics (hard-pure), Engineering and Medicine (hardapplied), Art and History (soft-pure), and Business and Law (soft-applied).

In terms of the variation in the vocabulary of pure and applied disciplines, the ASWL and its levels provided slightly higher coverage in the two applied sub-corpora $(91.07 \%$ and $90.92 \%)$ than in the two pure sub-corpora (89.46\% and $89.00 \%)$. This finding demonstrates the differences in the nature of applied and pure disciplines. Applied disciplines provide students with the training of specific skills and methods (Biber, 2006). Therefore, compared with the language in pure disciplines, the language in applied disciplines is closer to everyday language. Therefore, academic speech from the latter disciplines is likely to consist of a larger number of general high-frequency words than that of the former. As the ASWL also includes general high-frequency words, it is understandable why the ASWL and its levels provided slightly higher coverage in the applied sub-corpora than in the relevant pure sub-corpora. 
The variation in the vocabulary of hard and soft disciplines is apparent from the coverage of the ASWL levels in different sub-corpora. Levels 1 and 2 of the ASWL, which represent general high-frequency words, covered a slightly larger number of words in the soft subcorpora than in the relevant hard sub-corpora. Their coverage in the soft-pure sub-corpus $(86.37 \%)$ was higher than in the hard-pure sub-corpus $(85.87 \%)$ while their coverage in the soft-applied sub-corpus $(87.72 \%)$ was greater than in the hard-applied sub-corpus $(87.43 \%)$. In contrast, it was the other way around for the coverage of Levels 3 and Level 4 of the ASWL. These words covered a larger proportion of items in the hard-pure subcorpus $(3.59 \%)$ and hard-applied sub-corpus (3.64\%) than in the soft-pure sub-corpus $(2.63 \%)$ and soft-applied sub-corpus $(3.20 \%)$. These findings illustrate the differences in the nature of hard and soft disciplines and the teaching practice in these disciplines.

As found by Biglan (1973a, 1973b), the teaching of hard disciplines has a greater focus on informing students scientific facts, principles, and concepts of the material words. In contrast, the teaching of soft disciplines emphasizes helping students expand and deepen their existing knowledge. This teaching practice reflects the nature of the two disciplinary groups. According to Biber (2006), hard sciences are the disciplines of discovering, identifying, and describing new entities that do not have everyday references; therefore, they use a large number of words that have no commonplace synonyms (e.g., dextrinoid, electrophoresis, phallotoxins). In contrast, soft sciences deal with new perspectives of the concepts and entities from everyday experience; hence, many of the technical words in soft sciences can be explained by everyday language (Biber, 2006). For example, pedagogy can be explained as a teaching style. The difference between the nature of two disciplinary groups may be the reason why general high-frequency words play a more significant part in academic speech from soft disciplines than from hard disciplines while it is the other way around for general low-frequency words. It helps to explain why Levels 1 and 2 of the ASWL provided higher coverage in the two soft sub-corpora than in the two hard subcorpora. However, the fact that hard disciplines are more consistent than soft disciplines in terms of content and methods (Biglan, 1973a, 1973b) also means that hard sciences consist of more low-frequency words than soft sciences, and these low frequency words are more likely to be shared across multiple subject areas. For example, algorithm and analog are general low-frequency word families. The former appears at the $5^{\text {th }} 1,000 \mathrm{BNC} / \mathrm{COCA}$ word level while the latter is at the $25^{\text {th }} 1,000$ BNC/COCA word level. However, both 
occurred in 11 out of 12 hard subjects of the first academic spoken corpus in the present study. The fact that hard disciplines have a large number of shared general low-frequency words may be the reason why Levels 3 and 4 of the ASWL provided higher coverage in the two hard sub-corpora than in the two soft sub-corpora.

The slight differences in the coverage of the ASWL in pure versus applied and hard versus soft disciplinary sub-corpora demonstrate the variation in lexical items across disciplines (Durrant, 2014, 2016; Hyland \& Tse, 2007). This then highlights the value of disciplinespecific word lists. Specialized vocabulary tends to occur more often in specialized texts (Chung \& Nation, 2004). Hence, compared with universal academic word lists, disciplinespecific word lists are better at drawing learners' attention to the most important words in specific areas and providing a shortcut to reduce the amount of learning (Nation, 2013). Also, learners are motivated to learn items from discipline-specific lists because they can clearly see the relationship between what they study in their English courses and their subject courses (Basturkmen, 2003; Harwood \& Petríc, 2011; Hyland, 2016).

\subsubsection{What are the similarities between vocabulary in different disciplines?}

While variation in the vocabulary of academic speech between disciplines leads us to favor discipline-specific lists, it does not mean that we should dismiss the idea of universal academic word lists. This study found a considerable number of shared items in academic speech from different disciplinary groups. The ASWL consists of 1,741 word families with high frequency, wide range, and even distribution in a range of academic speech. Although the list was derived from academic speech of 24 subject areas, it still provides around $90 \%$ coverage in the four sub-corpora. Moreover, the ASWL provided higher coverage in the two academic spoken corpora (around 90\%) than the non-academic spoken corpus (around $87 \%$ ) and the academic written corpus (around 82\%), which suggests that the ASWL better represents academic spoken vocabulary than non-academic, and written vocabulary.

Together, these findings indicate that there is a core vocabulary across academic speech of different disciplines. Dang and Webb (2014) found that the variation in the coverage of Coxhead's (2000) AWL across disciplines of academic spoken English was not as great as that reported in studies investigating the coverage of the AWL in academic written English (Cobb \& Horst, 2004; Coxhead, 2000; Hyland \& Tse, 2007). Although, in their analysis, Dang and Webb (2014) used an academic written word list to represent academic spoken vocabulary, their results support the findings of this study; that is, the similarities between 
vocabulary in academic speech of different disciplines are greater than the differences. The existence of a core vocabulary across academic speech of different disciplines supports the development of universal word lists such as Coxhead's (2000) AWL, Gardner and Davies'(2014) AVL, or the ASWL.

Universal academic word lists are beneficial in two ways. First, they have a wide application (Basturkmen, 2003, 2006; Coxhead \& Hirsh, 2007; Harwood \& Petríc, 2011; Hyland, 2016; Nation, 2013). They can be useful in contexts where learners plan to study different disciplines or have not yet identified their target disciplines. Additionally, they are suitable in the context where EAP teachers lack background knowledge of learners' specific disciplines. Also, they are appropriate in the interdisciplinary environment where it is unclear which specific discipline an academic subject belongs to. This argument is supported by Banister's (2016) findings that Coxhead's (2000) AWL, a universal academic word list, was widely used and perceived as a useful instrument for L2 learners from a wide range of subjects by EAP teachers.

Second, focusing L2 learners' attention on the shared items between multiple academic subjects allows them to comprehend the words in a range of disciplines and contexts (Nation, 2013). For example, the selection of ASWL words is based on frequency, range, and dispersion from a large, representative, and balanced academic spoken corpus. Therefore, the list can capture the lexical items that L2 learners are likely to encounter often not only in their specific subject areas (e.g., Engineering) but also in related subject areas (e.g., Mathematics, Physics).

It should be noted that drawing learners' attention to the core vocabulary does not mean a lack of focus on the discipline-specific meanings of a word. As suggested by Nation (2013), the core meaning and discipline-specific meanings should not be seen as different from each other. Knowledge of the core meaning provides an excellent scaffolding for the acquisition of discipline-specific meanings (Crossley, Salsbury, \& McNamara, 2010). Highly frequent meanings are more likely to be stored as separate entries in the brain while less frequent meanings are more likely to be inferred from the context. Therefore, knowledge of the core meaning of an academic word will help learners to gradually become aware of its discipline-specific meanings if they meet the word very often in texts from their specific disciplines. These multiple encounters of the words from universal academic 
word lists in different contexts help to enrich learners' knowledge of the specific senses and store them in their brains.

Taken together, the slight difference in the coverage of the ASWL across the four disciplinary sub-corpora indicates that there is still variation in lexical items across disciplines of academic spoken English. Yet, there is a core vocabulary across these disciplines. Hence, there are no one-size-fits-all academic word lists. The more specialized a word list, the narrower its application, but the greater its benefit in a specific context (Coxhead \& Hirsh, 2007). Depending on the particular teaching and learning context, either a discipline-specific list or a universal academic word list can be a valuable resource for L2 learners. In the English for Specific Academic Purposes context where learners have highly specific needs, and plan to study the same discipline (e.g., hard disciplines) or even the same subject area (e.g., Medicine), discipline-specific lists may better serve learners' needs than universal academic word lists. The similarities in the learners' academic discipline may make it easier for teachers to focus on more specialized vocabulary in a specific discipline. However, in the English for General Academic Purposes contexts where learners are more heterogeneous or unclear about their discipline areas, it is usually challenging for EAP teachers to satisfy the specific needs of every learner in their classes. Hence, a universal academic word list is more practical. This idea is supported by Hyland (2016), who points out that the general and specific EAP approaches should be seen as ends of a continuum rather than a dichotomy. In other words, specificity in word list construction should be implemented with flexibility with the consideration of the circumstances of particular students in a class.

\subsubsection{What sources of information should be used in word list validation?}

So far in this section we have discussed how word lists can match learners' proficiency and academic majors. In this section, we will examine what factors are needed in word list validation. Given the close relationship between lexical coverage and comprehension (Laufer, 1989; Laufer \& Ravenhorst-Kalovski, 2010; Schmitt, Jiang, \& Grabe, 2011; van Zeeland \& Schmitt, 2013), most studies into word list validation used lexical coverage in corpora as the sole criterion to assess the value of a word list. Brezina and Gablasova (2015) even claim that, if the statistical measures are powerful enough, it is possible to develop a reliable list from corpora without human judgments. Nation (2012, 2016), however, argues that, using objective criteria (e.g., range, frequency, and dispersion) as the 
main criteria in word selection but adjusting the results by using other subjective criteria makes corpus-based word lists more useful and suitable for the target users. While Nation's (2012, 2016) view is sensible, two important questions are: what other data can supplement the information from corpora in word list validation, and how strong are the relationships between these sources? This thesis confirms that corpora are reliable resources for word list validation, but also indicates that information from teachers and learners is useful for evaluating the suitability of corpus-based word lists for L2 classrooms.

To begin with, Studies 1 and 2 of this thesis revealed that there were statistically significant correlations between lexical coverage from corpora with teacher perception of the usefulness and learner knowledge of the words from Nation's (2012) BNC/COCA2000 and Brezina and Gablasova's (2015) New-GSL. This indicates that items from these corpusbased lists of general high-frequency vocabulary are likely to be known by L2 learners and perceived as being useful by teachers. This finding is consistent with those from earlier studies. Research on vocabulary levels of L2 learners reported that these learners tend to know more items at higher frequency levels than those at lower frequency levels of corpusbased word lists (e.g., Laufer, 1998; Nguyen \& Webb, 2016; Nurweni \& Read, 1999; Stæhr, 2008; Webb \& Chang, 2012). Moreover, Ellis and colleagues found a significant correlation between teacher perception of instructional value and objective information from corpora (e.g., frequency, mutual information) of the items from their core Academic Formulas List (AFL) (Ellis, Simpson-Vlach, \& Maynard, 2008; Simpson-Vlach \& Ellis, 2010). Banister (2016) investigated the teaching practice, beliefs, and attitudes of teachers of academic English towards the pedagogical value of Coxhead's (2000) corpus-based AWL. Their results showed that, in general, the AWL was widely used and perceived as a useful teaching and learning tool by these teachers. Together the findings of the present study and previous research confirm that corpora provide a reliable way to develop and validate word lists for L2 learners.

Furthermore, this thesis demonstrates that information from corpora supplemented with information from teachers and learners has value in developing a pedagogical word list for L2 learners. As shown in Study 2, although lexical coverage was significantly correlated with teacher perception of word usefulness $(r=.23)$ and learner vocabulary knowledge $(r=$ .20), the correlations were small. There are two possible reasons. First, the target words in the learner Yes/No tests and the teacher surveys were peripheral items rather than shared 
items between Nation's (2012) BNC/COCA2000 and Brezina and Gablasova's (2015) New-GSL. Perhaps, the correlations would have been higher if the shared items had been examined. Second, lexical coverage is based on the analysis of the natural language occurrence and, thus, not all the words suggested by the corpora are suitable for L2 learning and teaching contexts (Milton, 2007, 2009; Nation, 2016). This is evident from the comparison between the New-GSL and the BNC/COCA2000. As mentioned in Section 6.2.2 above, the development of the New-GSL was solely based on the information from corpora. In contrast, the word selection of the BNC/COCA2000 also involved subjective judgements to ensure that the list suits L2 learning and teaching purposes. Therefore, despite the higher coverage provided by its most frequent headwords, the New-GSL always had a smaller proportion of headwords known by the learners and perceived as useful by the teachers than the BNC/COCA2000.

In contrast, there was a strong and significant correlation between teacher perception of word usefulness and learner vocabulary knowledge $(r=.67)$. This means that the words perceived as useful by teachers are more likely to be known by learners. The close relationship between teacher perception and learner vocabulary knowledge was also reported in previous studies that examined teachers' perception from the aspect of lexical difficulty (Brutten, 1981; McNeill, 2005). These studies showed that the words predicted as being problematic often actually caused difficulty for L2 learners.

The close relationship between the information from teachers and learners can be explained by the nature of teacher perception and L2 learning and teaching practice. Teachers' perception and practice are mutually informing (Borg, 2003, 2006). If teachers think the words are useful or cause difficulty for their learners, they are likely to create learning opportunities to support their learners' acquisition of these words. L2 learners, especially EFL learners, generally have less exposure to the target language than first language (L1) children (Ellis, 2002a,2002b; Nation, 2001). For many of them, classrooms may be the main environment to gain input and practice the target language (Guo, 2011; Lau \& Rao, 2013). Therefore, the words that teachers introduce and the guidance that they provide to learners in their in-class and out-of-class learning activities have a great impact on learners' vocabulary acquisition. In other words, the words that teachers think need special support are likely to be acquired by learners. In turn, the teaching practice may make teachers to change their perception accordingly (Borg, 2003, 2006). If teachers think that a certain 
word is useful and plan to introduce it, but then find that their learners already know the word, they may then focus less on this word. In contrast, during their teaching practice, if teachers notice that their learners do not know the word that they assume that learners already know, they may provide more instruction on this word.

To sum up, this thesis revealed that lexical coverage, teacher perception of word usefulness, and learner vocabulary knowledge are related. However, the relationship between teacher perception of word usefulness and learner vocabulary knowledge are stronger than those between each of these factors with lexical coverage. Based on these findings, it is suggested that corpora are reliable sources for word selection; however, to achieve a better outcome when introducing corpus-based word lists to L2 classrooms, the information from corpora should be supplemented with the information from teachers and learners. Considering that most studies into word list validation used lexical coverage from corpora as the sole criterion, to better serve the L2 learning and teaching, as well as relying on large and representative corpora, it is useful for list developers to involve teachers and learners in word list validation.

\subsection{How important is teachers' familiarity with learners' characteristics in $L 2$ vocabulary teaching and learning?}

So far this chapter has looked at the themes related to the two core concepts of vocabulary studies: general high-frequency words and word list construction. In this section, we will focus on teacher perception, an underexplored but potential area of vocabulary research. Teacher cognition can be looked at from different aspects (Borg, 2003, 2006). Covering all of them is beyond the scope of a single thesis. Therefore, this section only focuses on the most salient factor emerging from the results of this thesis; that is, teachers' familiarity with learners' characteristics.

The very few vocabulary studies that investigated teacher perception suggested that teacher perception of word value and learner vocabulary knowledge are related. However, what is less transparent from these studies is how this relationship changes among teachers and learners in different contexts. This thesis reveals that the more familiar teachers are with their learners, the closer the relationship between their perceptions of word usefulness and their learners' vocabulary knowledge. Regardless of the learner groups, Vietnamese L1 
teachers always had the highest correlations between perceptions of word usefulness and learner vocabulary knowledge ( $r$ from 0.58 to 0.71 ). Next came the various L1 teachers $(r$ from 0.52 to 0.65 ). The English L1 teachers had the lowest correlations ( $r$ from 0.45 to $0.56)$.

One possible reason for this finding is teachers' levels of familiarity with learners' L2 learning experience and contexts. Of the three groups of teachers, the Vietnamese L1 teachers had the closest L2 learning experience with the learner participants. To some extent, these teachers are advanced Vietnamese EFL learners. Moreover, they had experience teaching Vietnamese EFL learners in Vietnam. As a result, they were more familiar with the learning contexts of the learner participants than the other groups of teachers. The various L1 teachers ranked second in terms of familiarity with the learner participants' L2 learning experience and contexts. Like the learner participants, these teachers learned English as an L2, and they were likely to acquire English as adults. Additionally, these teachers taught English in their home countries (e.g., China, Indonesia, Venezuela, and Kenya). Therefore, their learners had similar learning contexts as the learner participants; that is, they studied English in their home countries and were taught by teachers sharing the same L1 with them. The English L1 teachers were the least familiar with the L2 learning experience and context of the learner participants. They learned English as the L1 when they were children. Although they may have learned other languages, these experiences may still be distant from the L2 learning experience of the learner participants. Moreover, the learning contexts of the learners that these teachers experienced teaching were more varied. Some of their learners had similar learning contexts as the learner participants. Yet, the others had different learning contexts; that is, they studied English in English speaking countries and were mainly taught by teachers who did not share the same L1 with them. Teachers' prior language learning experience and contextual factors play a significant part in determining teacher perception of vocabulary learning and teaching (Gao \& Ma, 2011; Gerami \& Noordin, 2013; Lau \& Rao, 2013; Nural, 2012; Rahimi, 2014; Zhang, 2008). Therefore, it is understandable why Vietnamese L1 teachers ranked first, various L1 teachers ranked second, while English L1 teachers ranked last in terms of correlation between their ratings and the learner vocabulary knowledge. 
The familiarity with learners' L1 is another reason why the Vietnamese L1 teachers had higher correlations between perceptions of word usefulness and learner vocabulary knowledge than the other teacher groups. Research has confirmed the significant role of L1 in facilitating L2 learning in general and vocabulary in particular (Ma, 2012; Medgyes, 1992; Schmitt, 2008, 2010). Teachers sharing the same L1 with their learners are better at identifying the sources of lexical difficulty than teachers who do not share or are unfamiliar with learners' L1 (Ellis, 2002; Ma, 2012; McNeill, 2005). Moreover, they can make use of their L1 to explain different language patterns if communication in English fails (Ellis, 2002). Given these benefits of L1, it is understandable why Vietnamese L1 teachers had higher correlations with the learners' vocabulary knowledge than other groups.

In sum, this study shows that the greater the teachers' familiarity with learners and the learning contexts, the better they can judge which words are useful for learners. It then supports the suggestion that teachers' knowledge of learners and learning contexts is fundamental in L2 vocabulary learning and teaching (Farrell, 2015; Ma, 2012; Medgyes, 1994; Richards, 2010; Richards \& Farrell, 2005).

\subsection{Rationale for the next chapter}

This chapter has discussed the three main themes drawn from the findings of the thesis. Two of them (high-frequency words and word list construction) are core issues in vocabulary studies while the other (teacher perception) is an underexplored but potential area for further vocabulary research. The next chapter concludes the thesis by looking at the theoretical, pedagogical, and methodological implications of this thesis as well as its limitations and direction for future research. 


\section{Chapter 7 - Conclusion}

\subsection{Introduction}

To address the lack of research on vocabulary in academic speech, this thesis explored the nature of vocabulary in academic spoken English with three linked studies. Given the common assumption that second language (L2) learners already know general highfrequency words before moving to academic words, the first two studies identified the most suitable general high-frequency word list for these learners from the perspectives of corpus linguistics, teachers, and learners. Also, they determined the relationships among these three sources. The last study involved developing an Academic Spoken Word List (ASWL) which is adaptable to L2 learners' proficiency and using this list to explore the nature of academic spoken vocabulary. These three linked studies provide valuable contributions for theory, methodology, teaching, and learning.

This chapter begins with the theoretical contributions of the thesis (Section 7.2). It is followed by the methodological contributions (Section 7.3), and pedagogical implications (Section 7.4). The chapter ends with the limitations, direction for future research, and conclusion (Sections 7.5 to 7.7 ).

\subsection{Theoretical contributions}

The present research has four main theoretical contributions. First, it sheds light on the nature of academic spoken vocabulary, an underexplored area of vocabulary research. The study indicates that general high-frequency words, especially the most frequent 1,000 words, play a much more significant part in academic spoken English than in academic written English. Importantly, the vocabulary in academic spoken English varies to some degree according to disciplines. Yet, a core vocabulary exists across multiple disciplines and is presented in the ASWL. Thus, this study is a valuable starting point for further research investigating academic spoken vocabulary such as studies examining vocabulary load, testing, incidental learning, and specialized word lists.

Second, this is the first study that has compared a large number of general high-frequency word lists from different perspectives (corpora, teachers, and learners) using multiple approaches to data analysis. It provides solid evidence that Nation's (2012) 
BNC/COCA2000 is the most suitable general high-frequency word list for L2 learners. This finding is meaningful given that general high-frequency vocabulary is a core concept in L2 vocabulary research, learning, and teaching.

Third, the present thesis indicates that teachers' familiarity with learners' characteristic such as L2 learning experience, contexts, and first language (L1) is correlated with L2 vocabulary knowledge. Considering the lack of research in teachers' perception of general high-frequency word lists in both vocabulary research and teacher cognition research, the findings of this thesis provide valuable contributions to the literature in both fields.

Fourth, although earlier research implies that information from corpora, teachers, and learners is related, the strength of these relationships was unclear. This study found that these factors are related; however, the relationships between lexical coverage with teacher perception of word usefulness and with learner vocabulary knowledge are small while the relationship between learners and teachers is large. This finding then provides further insight into the relative value of corpora, teachers, and learners in L2 vocabulary learning and teaching.

\subsection{Methodological contributions}

The present study has made three valuable contributions in methodology related to word list development and validation. These contributions are discussed in turn below.

\subsubsection{How to make an academic word list adaptable to learners' proficiency?}

The biggest methodological contribution is the way of making a word list adaptable to learners' proficiency. The ASWL was created from scratch but was divided into levels to match learners' vocabulary levels. In this way, it can make the best use of the strengths of the two approaches toward identifying academic words: creating the list from scratch (Gardner \& Davies, 2014) and making it appropriate for learners at a certain level (those who know, for example, the general high-frequency words) (Coxhead, 2000; Xue \& Nation, 1984). No previous research on developing academic word lists has looked at the issues from this perspective.

Creating the list from scratch allows the list to avoid the limitations related to ready-made lists of general high-frequency vocabulary. Also, it focuses learners' attention towards the most important words in academic speech, especially the items whose occurrences in 
general conversation are not frequent enough for incidental learning to happen through listening to academic spoken discourse. Creating the ASWL from scratch meant excluding 714 general high-frequency words (e.g., delicious, pudding, grin) that do not have high frequency, wide range, or even distribution in academic speech and including 455 words at lower frequency levels that occur often in a wide range of academic speech. Additionally, creating the ASWL from scratch results in a list with a more manageable size (1,741 items) but higher coverage than the most frequent 2,000 BNC/COCA words.

Meanwhile, dividing the lists into levels takes learners' existing knowledge of general vocabulary into account and avoids repeatedly teaching and learning known items. However, while previous studies made a fixed benchmark of the number of general words that every learners should acquire before learning items from academic word lists, the ASWL was graded into levels according to Nation's (2012) BNC/COCA lists so that learners can start learning the ASWL items that are relevant to their existing vocabulary levels. In brief, the development of the ASWL provides a useful way to make corpus-based word lists more suitable to L2 learning and teaching.

\subsubsection{How to validate corpus-based word lists?}

A second methodological contribution of this thesis is its extensive and intensive validation of word lists. In terms of breath, this research has highlighted the importance of combining information from different sources (corpora, teachers, and learners) in word list validation. Corpora provide a reliable way to identify the lexical items that learners are likely to encounter and use in their future. Meanwhile, the information from teachers and learners allows list developers to be better aware of the contextual and circumstantial realities of a language classroom, and achieve a better outcome when introducing their corpus-based word lists to L2 classrooms. Let us now look at how these sources should be used in word list validation.

To begin with, the validation should be based on large and representative corpora to achieve a thorough picture about the nature of a word list. The ASWL was validated in three corpora (an academic spoken corpus, a non-academic spoken corpus, and an academic written corpus), each of which focused on validating different aspects of the ASWL and had a similar size as the corpus from which the list was developed (around 13-million running words). Noticeably, apart from having the same size, the academic spoken corpus 
used to validate the ASWL also had the same structure as the one used to develop the list. It included academic speech from a wide range of subject areas that represent at least seven varieties of English. In each corpus, these materials were distributed into four equally-sized disciplinary sub-corpora: hard-pure, hard-applied, soft-pure, and soft-applied. The materials represent four kinds of classroom speech events that English for Academic Purposes (EAP) learners are likely to encounter in their academic study: lectures, seminars, labs, and tutorials. Likewise, the validation of the four general high-frequency word lists was based on nine spoken and nine-written corpora that represented a wide range of spoken and written discourse and 10 different varieties of English. It should be noted that corpora have been widely used as a means of validating academic written word lists (Coxhead, 2000; Gardner \& Davies, 2014) and lists of academic formulas (Simpson-Vlach \& Ellis, 2010) as well as comparing general high-frequency word lists (Brezina \& Gablasova, 2015; Browne, 2014; Gilner \& Morales, 2008; Nation, 2004; Nation \& Hwang, 1995). Yet, no earlier research has validated academic spoken word lists and general high-frequency word lists in a larger number of corpora with larger sizes and greater degree of representativeness than the present study.

Similar to the use of corpora to validate word lists, to provide a more accurate assessment of word lists, the teachers and learners involved in the validation should be large in number and have a great degree of variety. The validation of the general high-frequency word lists involved the participation of 25 English L1 teachers, 26 Vietnamese L1 teachers, 27 various L1 teachers, and 275 Vietnamese English as a Foreign Language (EFL) learners. The teachers represented a wide range of L1 backgrounds, teaching contexts, and years of teaching experience. Also, they were experienced in teaching English to L2 learners from beginners to advanced levels. The learners represented different proficiency levels, university levels, and different majors.

In terms of depth, the information from each source (corpora, learners, and teachers) should be analyzed from different angles to yield a thorough understanding about the nature of the list. It can be seen clearly from the validation of the four general high-frequency word lists in the present thesis. The data from corpora were analyzed in two ways: average coverage and coverage provided by the most frequent items. Similarly, the analysis of the teacher and learner data was based on the examination of both the whole list and the most frequent items in each list, and a range of criteria were used as indicators of word usefulness and 
vocabulary knowledge. In brief, this study highlights the importance of employing different methods of evaluation both in breadth and depth for a comprehensive assessment of word lists.

\subsubsection{How to compare word lists of different units of counting?}

The third methodological contribution is to use headwords as the unit to compare lists of different units of counting. This way minimizes the difference in the number of items in each list because other members of the families were not included. Moreover, it ensures that the nature of the list does not significantly change because headwords are usually the most frequent members of the word families. Furthermore, using headwords reflects L2 learning and teaching practice. That is, learners and teachers usually receive lists of headwords without their inflections and derivations and may choose headwords to teach and learn first. Additionally, using headwords reflects the principles behind the selection of unit of counting of each word list. Given the variation in the unit of counting of current word lists, the approach taken to compare the four general high-frequency word lists in the present thesis has methodological value.

\subsection{Pedagogical implications}

The previous sections have presented the theoretical and methodological implications of this thesis. This section discusses the thesis contributions for L2 vocabulary learning and teaching. It has three sub-sections, each of which corresponds to each theme discussed in the Discussion chapter. The first sub-section provides some suggestions about developing learners' knowledge of the BNC/COCA2000 and ASWL words to improve their comprehension of academic spoken English. The second sub-section presents the implications related to word list development and implementation. The last sub-section suggests how teachers of English can enhance their knowledge of learners' characteristics in L2 vocabulary learning and teaching.

\subsubsection{Developing learners' knowledge of the BNC/COCA2000 and ASWL words}

The Discussion chapter indicates that knowledge of the items from the BNC/COCA2000 and ASWL lists is significant for L2 learners' comprehension of academic spoken English. However, it is important to note that working with these word lists is not simply rote learning. Instead, these items should be learned in a principled way. This section provides some suggestions on how teachers can help their learners to determine the learning 
sequences of the BNC/COCA2000 and ASWL words and to develop their knowledge of these words.

\subsubsection{Determine the learning sequence}

At the beginning of an English courses, depending on learners' existing levels of general vocabulary and their specific learning and teaching contexts, learners and teachers can use the sequence in Figure 7.1 as a guide to set the long-term learning goals for the learners. Given the wide use of the BNC/COCA lists in vocabulary studies, using these lists to guide the learning sequence makes it possible for teachers, course designers, and material writers to incorporate the findings of the present study with the findings of other studies related to the BNC/COCA lists to organize a more effective vocabulary learning program.

Figure 7.1. Vocabulary learning sequences for different groups of learners

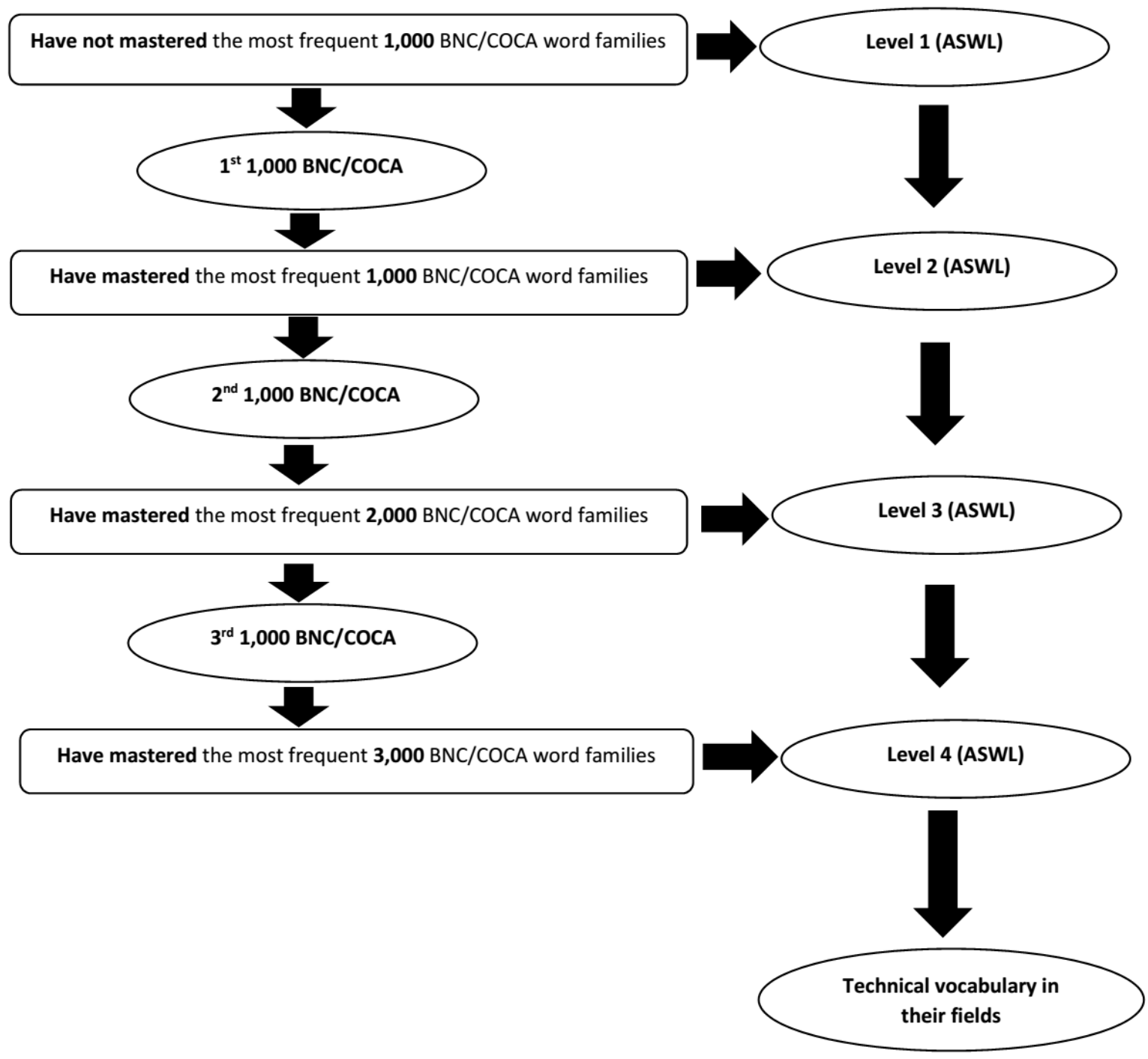


The sequence shown in Figure 7.1 also helps learners and teachers avoid repeatedly teaching and learning known items, and makes it easier for teachers and course designers to incorporate teaching the ASWL to groups of learners with different vocabulary levels and learning purposes. Let us take learners who have not mastered the most frequent 1,000 $\mathrm{BNC} / \mathrm{COCA}$ word families as an example. If they want to go straight to the lexical items that appear frequently in their academic studies, they can start learning the ASWL at Level 1. However, if they would like to acquire the items that are useful for general conversation before moving to the words occurring frequently in their academic disciplines, they can learn items from the BNC/COCA word lists first. Once they are satisfied with their levels of general vocabulary, they can start learning the ASWL at the level which is relevant to their general vocabulary level at that time.

While the ASWL levels provide guidance in setting long-term learning goals for L2 learners, its sub-lists can assist learners and teachers in setting short-term learning goals. Each level of the ASWL was divided into a sub-list of function words and sub-lists of lexical words. Each sub-list of lexical words contains 50 or fewer items (see Appendices 17-20). The ASWL sub-list of lexical words with manageable sizes make it possible for learners and teachers to set short-term learning goals and easily incorporate the ASWL in their language learning program. This idea is supported by Dang and Webb (2016) and Nation (2016). Moreover, teaching and learning the ASWL according to the rank order of sub-lists ensures that the most useful items are learned first as well as allowing programs to prepare a curriculum that covers all sub-lists, and avoids teaching the same items between courses (Coxhead, 2000; Dang \& Webb, 2014).

Distinguishing between lexical words and function words in the ASWL has pedagogical value. It takes into account the difference in the way learners deal with different kinds of vocabulary (Nation, 2016). In a text, lexical words are more salient than function words; therefore, the way to deal with lexical words should be different from the way to deal with most function words (Carter \& McCarthy, 1988). It will be best to sequence the teaching of lexical words according to their frequency. However, it is more reasonable to incorporate teaching function words with other components of language lessons due to their lack of salience in the text. 
It should be noted that the levels and sub-lists should be treated as a guide rather than a handbook for teachers and learners to strictly follow. Moreover, although the ASWL consists of 1,741 word families, this does not mean that learners should learn only 1,741 ASWL headwords. Similarly, although the ASWL and BNC/COCA2000 are presented in the format of word lists, this does not mean that these lists should be learned and taught solely by decontextualized methods (Coxhead, 2000). Knowing a word involves many types of vocabulary knowledge (Nation, 2013). Therefore, once the learning goals have been set, it is important for teachers to organize learning opportunities for learners to acquire, consolidate, and expand on their knowledge of the target words in a meaningful way. This does not mean that the target words must be the sole content of a language course, but they should be considered an integral part of language learning activities both within and beyond classrooms. To provide learners with a continual development of the vocabulary knowledge, initial focus should be on the form and meaning relationship, and then on other aspects of vocabulary knowledge. The recommendations related to each point are presented in turn below.

\subsubsection{Focus on the form and meaning relationship first}

Knowledge of form and meaning of the BNC/COCA2000 and ASWL words should be the initial focus because this aspect acts as the foundation for the development of other aspects of vocabulary knowledge (Gardner, 2013; Webb \& Chang, 2012). Helping learners to acquire the form and meaning relationship of the BNC/COCA2000 and ASWL words involves developing their knowledge of (1) the written and spoken forms of these words, and (2) linking it to the core meaning and discipline specific meanings of the words.

Research has shown that some learners have difficulty matching the written form of the word with its spoken form (Chang, 2007). There are two possible reasons for this. First, some learners may acquire the words mostly through listening, and, therefore, lack experience with the written form while others learn vocabulary mostly through reading and may not be very familiar with its spoken form (Coxhead, 2006). Teachers can address this problem by providing learners with plenty of opportunities to encounter and use the spoken and written forms of the target words in different contexts (Lynch, 2011). Second, learners may be unable to match the words they hear with the information stored in their memory (Goh, 2000). To deal with this, teachers can make use of pre-listening activities such as brainstorming the words related to the listening topics or class-based discussion about the 
listening topics. These activities activate learners' background knowledge of the topics before they start the listening task. Meanwhile, some target words may come up during these activities, which provides teachers with opportunities to see how well their learners know the words and decide if they need further teaching or learning of these words (Coxhead, 2006).

With respect to knowledge of meanings, both the BNC/COCA2000 and ASWL words have many meanings which may cause difficulties for learners in recognizing the form and meanings relationship. Therefore, teachers should help learners to acquire the core meaning first and then guide them to gradually acquire the discipline-specific meanings.

To raise learners' awareness of the core meaning, teachers should provide them with opportunities to see how the target words are used in different contexts. For example, they can provide learners with different spoken or written extracts which contain the target words or instruct learners to look up the entry of a target word in dictionaries or concordances (Flowerdew, 1993; Nesi \& Basturkmen, 2006). There are a number of concordance resources that represent different kinds of discourse that teachers can make use of. Concordances for a range of corpora can be found at Tom Cobb's (n.d.) website and Mark Davies's website (http://corpus.byu.edu/corpora.asp). More sources can be found in Appendix 21. When learners examine these examples, teachers can encourage them to identify the similarities in the meanings of the target words and discuss them with their teachers and peers. In this way, the core meanings of the words will become more salient to learners. Once learners have acquired the core meaning, teachers can follow similar steps to help learners expand their knowledge to discipline-specific meanings by drawing learners' attention to the meaning of the words in their specific disciplines. Actively thinking about the target word and getting feedback from their teacher and peers help learners to acquire and remember the meaning better (Coxhead, 2006, 2012). Gradually directing learners' attention from the core meaning to discipline-specific meanings consolidates learners' knowledge of the core meaning while enabling them to expand their knowledge of discipline-specific meanings of the target words.

\subsubsection{Develop other aspects of word knowledge}

Once learners have mastered the form and meaning relationship of a target word, teachers should help learners gradually develop knowledge of other aspects of the target words both 
receptively and productively. This can be done by designing learning activities and materials for learners to repeatedly encounter and use these words in different contexts related to their target subject areas. This way provides learners with meaningful contexts and rich learning opportunities when working with word lists (Coxhead, 2000; Coxhead \& Hirsh, 2007). Nation's (2007) four strands (meaning-focused input, meaning-focused output, language-focused learning, and fluency development) provides a useful framework for organizing learning opportunities. This section discusses how each strand can be applied to help learners develop different aspects of the BNC/COCA2000 and ASWL words.

\section{Meaning-focused input and meaning-focused output}

Meaning-focused input means that learners acquire the target words by using the language receptively through listening and reading while meaning-focused output involves learning words by using language productively through speaking and writing.

To ensure that the learning from meaning-focused input and from meaning-focused output happens, teachers should consider the following conditions. First, they should provide learners with plenty of opportunities to read, listen, write, and speak about the topics related to learners' target disciplines and interests. Second, they should ensure that learners are familiar with $95 \%-98 \%$ of the words in the input and output. The large amount of input and output ensures that learners will have multiple meetings with the target words in different contexts both receptively and productively. The familiar and interesting topics and the large proportion of known words in the input and output create the environment in which learners can read and listen with pleasure and notice more about the target words. Similarly, these conditions encourage learners to speak and write more.

To select the spoken and written input for their learners, teachers can make use of open courseware such as those from the Massachusetts Institute of Technology, Stanford University, Yale University, and University of California, Berkeley (see Appendix 22 for the links to these sources). These websites provide free reading materials as well as videos and audios of lectures with transcripts and subtitles on a wide range of subject areas. Another useful resource for EAP learners to get used to academic spoken vocabulary is TED (Technology, Entertainment, and Design) Talks (Coxhead \& Walls, 2012) (see Appendix 22 for the links to this source). This website contains short monologues given by experts who try to explain scientific knowledge on a wide range of topics to a lay public. 
The videos of the talks are accompanied with transcripts, captions, as well as different kinds of activities to support listening comprehension.

Depending on their students' disciplines, vocabulary levels, and interests, teachers can choose suitable topics and adapt materials from these sources for their students. In terms of topics, Coxhead and Walls (2012) and Dang and Webb (2014) provide useful information about the lexical demands of academic spoken texts from different disciplines. To adapt the materials to learners' levels, Webb and Nation (2008) provided detailed instruction on how teachers can use Nation, Heatley, and Coxhead's (2002) RANGE program to measure the lexical load of learning materials and to adapt the materials to suit learners' vocabulary level. Other free programs that have a similar function to RANGE are Laurence Anthony's (n.d.) AntwordProfiler and Tom Cobb's (n.d.) vocabprofiler at Lextutor. For spoken texts, teachers can adjust the speed rates of the recording or organize pre-listening activities to support listening comprehension (see some examples of these activities in Section 7.4.1.2 above).

\section{Language-focused learning}

While the main focus of attention in the meaning-focused input and meaning-focused output strands is on information, the language-focused learning strand draws attention to BNC/COCA2000 and ASWL words themselves. Language-focused learning is necessary because it enables students to gain a large amount of knowledge in a limited period of time (Coxhead, 2000). Also, it directs learners' attention to important but less frequent items or lexical features which are not likely to be acquired from normal acquisition due to the lack of repetition (Coxhead, 1998). Language-focused learning only happens if teachers help their students deliberately pay attention to these features, and process them in a thoughtful way, and if they create repeated opportunities for the students to pay attention to the same language features throughout the course. Depending on the learning burden of a certain word, teachers can focus on certain aspects of vocabulary knowledge in language-focused learning activities. For instance, they can let learners practice pronunciation and stress patterns so that they can recognize and produce the words correctly in spoken discourse (Murphy \& Kandil, 2004). They can also help learners build up their knowledge of words expressing stances and discourse markers to improve their comprehension of academic speech. 


\section{Fluency development}

The fourth strand is fluency development. It involves all four skills: listening, speaking, reading, and writing. This strand does not aim to enable students to learn new words, but to become more fluent in using the items they already know. This is a movement towards high-level proficiency with vocabulary. Nation (2013) suggests that, for learning from fluency development activities to happen, teachers should satisfy the following conditions. First, they should ensure that their students know all the words in the task and are familiar with the topics. Second, they should require the students to perform the task at a faster speed than normal. Third, they should make sure that the students' focus is on receiving and conveying meaning. Fourth, they should involve a large amount of input and output in the activities.

For example, learners can read and listen to texts related to their target disciplines several times. Each time they work on understanding the texts at different levels. For instance, the first time, they might read and listen to the text to understand the main ideas. For the second time, they might try to understand the author's ideas and arguments to add new information to their knowledge and support their viewpoints. The third time, they might read and listen to the text to synthesize the ideas in writing or speaking. For reading tasks, learners can set the time to complete the reading. For listening tasks, the speed of the spoken input should increase over the time. In terms of fluency in writing activities, learners can write on topics related to their familiar discipline-specific subjects. They can write many drafts and try to increase their writing speed over time. Similarly, for fluency speaking tasks, learners can participate in debates, group discussion, and seminar presentations in which they talk about the topics they are familiar with.

It is important to note that each strand contributes to the development of vocabulary knowledge in a different way and should be given one quarter of the course time. Teachers can find more examples of four strand activities suggested by Nation (2012), Nation and Yamamoto (2012), and Coxhead (2006, 2014). Moreover, they can make use of Laufer and Hulstijn's (2001) Involvement Load Hypothesis and Nation and Webb's (2011) Technique Feature Analysis to evaluate the relative effectiveness of vocabulary learning activities. 
In brief, this section highlights that helping learners to learn the BNC/COCA2000 and ASWL words does not mean that rote learning should occur from the beginning to the end of these lists. Instead, items from these lists should be learned in a principled way.

\subsubsection{Combining information from corpora, teachers, and learners in word list implementation}

The previous chapter has shown that corpora are realiable sources for word list construction; therefore, curriculum developers, material designers, teachers, and learners should make good use of corpus-based word lists such as Nation's (2012) BNC/COCA and the ASWL to sequence their vocabulary learning and teaching (Schmitt, 2008). However, given the important roles of teachers and learners in L2 vocabulary learning and teaching and the limitations of corpora, it is also essential for curriculum developers, material designers, teachers, and learners to keep two things in mind when using corpus-based word lists.

First, each word list may have its own principled applications. Therefore, before using a corpus-based word list, people should understand clearly the principles behind its development and decide whether the list matches their learning and teaching purposes. For example, the ASWL was developed with the aim to suit English for General Academic Purposes classes for L2 learners who are heterogeneous in terms of disciplinary areas or are unclear about their disciplinary areas. Thus, in more specialized contexts (e.g., English for Specific Academic Purposes or English for Specific Purposes) where all learners plan to study, for instance, hard subjects or Medicine, a discipline-specific spoken word list may be more appropriate than the ASWL. Moreover, the ASWL focuses on enhancing learners' comprehension of academic spoken English rather than academic written English. Hence, in a context where L2 learners mainly need to comprehend academic reading materials and have little need for comprehending academic spoken texts, academic written word lists (e.g., Coxhead, 2000; Gardner \& Davies, 2014), or discipline-specific written word lists (e.g. Coxhead \& Hirsh, 2007; Hsu, 2013; Liu \& Han, 2015; Wang, Liang, \& Ge, 2008; Ward, 2009) may be more relevant than the ASWL.

Second, corpus-based word lists focus on what is useful for learners based on which words learners are expected to encounter and use often (Nation, 2016). Hence, they just provide suggestions about the important vocabulary that learners should focus on as well as guiding 
the sequence vocabulary learning for teaching and designing courses and materials. Depending on the specific teaching and learning contexts and the characteristics of users, word lists and their sub-lists should be applied with flexibility. In other words, careless application of corpus-based word lists without a clear understanding of their nature may result in unexpectedly negative learning and teaching outcomes.

\subsubsection{Enhancing teachers' knowledge of learners in vocabulary teaching}

The previous chapter also highlights the importance of teachers' familiarity with learners' characteristics. This finding provides two implications for teachers of English. First, these teachers should be aware of the impact of contexts and prior language learning experience on their perception. Teachers whose L1 is not English should make use of their past experience as an L2 learner to provide learners with necessary support at different stages of their vocabulary development. As for English L1 teachers, they should be aware of the difference between their experiences of learning English and their learners' and actively examine their learners' difficulties, strengths, weaknesses, and needs (Braine, 1999; Medgyes, 1992). To do this, these teachers can consider their L2/L3 (third language) learning experience when planning their teaching. Another way is to talk to their learners or people who have the same or similar L1 background with their learners, or other colleagues who have experience teaching learners from the same or similar L1 background with their learners.

Second, this study highlights the value of L1 in L2 learning and teaching. Teachers who share the same L1 with the learners should make good use of their L1 to facilitate the L2 learning process. Meanwhile, teachers whose L1 is not the same as their students', especially those working in a monolingual learning context, should be aware of the linguistic features of the learners' L1 and how they are similar to and different from those of the learners' L2 (Medgyes, 1992). In this way, they can better identify the learning burden of the words. Swan and Smith (2001) provide useful information about the interlanguage features of learners from certain L1 backgrounds. Moreover, as mentioned above, talking with learners and people having the same L1 background as their learners may allow these teachers to better understand the differences and similarities between the learners' L1 and L2. 
So far in this chapter, the theoretical, methodological, and pedagogical implications of this thesis have been highlighted. The remaining sections of this chapter acknowledge the limitations of this thesis as well as pointing out the areas that need attention from future research.

\subsection{Limitations}

This study has a number of limitations. They can be classified into two groups. The first group consists of limitations related to common assumptions. Limitations in the second group are those caused by practical reasons. Each group is presented in turn below.

\subsubsection{Limitations related to common assumptions}

This thesis adopted several assumptions from earlier studies that could be questioned. First, the coverage figures in this thesis were based on the analysis of written transcription of spoken data with the assumption that learners are able to recognize the spoken forms of the words as well as proper nouns and marginal words. This assumption has been adopted by previous corpus-driven research investigating the vocabulary load of spoken texts (e.g., Coxhead \& Walls, 2012; Nation, 2006; Webb \& Rodgers, 2009a, 2009b). However it should be noted that, although L2 learners' aural and orthographic knowledge are closely related, the gap between their aural and orthographic knowledge may vary according to the learning context and learners' characteristics (Milton, 2009; Milton \& Hopkins, 2006; Milton, Wade, \& Hopkins, 2010). Similarly, Kobeleva (2012) found that previous knowledge of proper names has significant importance in listening comprehension; therefore, it may be optimistic to assume that proper nouns do not require previous knowledge when being encountered in listening (Nation, 2016).

Second, the Yes/No tests and the Vocabulary Levels Test (VLT) (Nation, 1983, 1990; Schmitt et al., 2001) were used as an indicator of learner vocabulary knowledge. These tests have been widely used to measure L2 learners' vocabulary levels (e.g., Henriksen \& Danelund, 2015; Schmitt et al., 2011; Webb \& Chang, 2012). However, these tests measure only one aspect of knowing a word, the written form-meaning relationship. In other words, if a word is indicated as known in a Yes/No test and the VLT, it does not necessarily mean that the participants possess other aspects of vocabulary knowledge. 
Third, following numerous previous word list studies (e.g., Coxhead \& Hirsh, 2007;

Gardner \& Davies, 2014; Lei \& Liu, 2016; Nation, 2006, 2012), the present research used Juilland and Chang-Rodrigues's (1964) D as a measure of dispersion so that its results can be compared with previous studies. However, Biber, Reppen, Schnur, and Ghanem (2016) found a great decrease in the sensitivity of D when the computations were based on a large number of sub-corpora.

Fourth, like most previous studies on developing word lists (Coxhead, 2000; Gardner \& Davies, 2014; Xue \& Nation, 1984), the current research only looked at the influence of lexical coverage on comprehension. However, there are many other factors affecting listening comprehension such as L1 listening ability (Vandergrift, 2006), background knowledge and topic familiarity (Bonk, 2000), learners' strategic ability (Bonk, 2000), speaker's accent (Goh, 2000; Matsuoka \& Hirsh, 2010; Richards, 1983), speech rate (Flowerdew \& Miller, 1992; Goh, 1999; Hasan, 2000), degree of concentration (Flowerdew \& Miller, 1992), visual aids (Flowerdew \& Miller, 1992), and interaction (Flowerdew \& Miller, 1992).

\subsubsection{Limitations caused by practical reasons}

For practical reasons, a number of decisions were made in this thesis that may result in some limitations. To begin with, only Vietnamese EFL learners participated in the second study. Although the use of teachers from different L1 backgrounds with teaching experience in different EFL/ ESL (English as a Second Language) contexts were used to triangulate the information from the learners, bias towards Vietnamese EFL learners is inevitable. Moreover, to make it possible to conduct the Yes/ No tests and surveys with a large sample of learners and teachers, not all BNC/COCA2000 and New-GSL words were included in the tests. Instead, only non-overlapping items from these lists were used as the test items.

Some other limitations were due to a lack of available resources. The first is related to the use of the VLT (Nation, 1983, 1990; Schmitt et al., 2001) to measure learners' vocabulary levels. West's (1953) General Service List is the general high-frequency baseword list in this test. Given that Nation's (2012) BNC/COCA2000 is the most suitable general highfrequency word list for L2 learners, a vocabulary levels test that was based on the $\mathrm{BNC} / \mathrm{COCA}$ lists should have been used to measure the learner level. However, when the 
present research was conducted, Webb, Sasao, and Ballance's (n.d.) New Vocabulary

Levels Test was still under validation while McLean, Kramer, and Beglar (2015) Listening Vocabulary Levels Test was designed specifically for Japanese EFL learners and has not had rigorous validation.

The second limitation is about the use of Coxhead's (2000) Academic Word List (AWL) and Gardner and Davies's (2014) Academic Vocabulary List (AVL) to represent academic written vocabulary in the comparison to the ASWL. These lists were chosen because they are the best known and most recent academic written word lists, and their validity has been confirmed. However, the differences in the principles behind the development of Coxhead's (2000) AWL, Gardner and Davies's (2014) AVL, and the ASWL may not provide a thorough picture about the differences between the most frequent, wide ranging, and evenly distributed items in academic writing and speech.

The third limitation is related to the second academic spoken corpus and the academic written corpus. Due to the lack of materials, these corpora were not as well balanced as the first academic spoken corpus used to develop the ASWL. While these validating corpora were much better than those used in earlier research on developing academic word lists, future research on academic word lists should develop two validating academic corpora (one spoken and one written) that mirror the structure of the academic corpus from which the academic word list is developed. In this way, researchers can compare the coverage of the ASWL in different disciplinary groups of academic speech and academic writing.

The last limitation is the use of the RANGE program (Nation et al., 2002) for the analysis. This program is a common instrument to analyze the vocabulary load of texts. However, RANGE was unable to distinguish between homographs; therefore, the present study is inevitably affected by the limitation of this program.

\subsection{Directions for future research}

The findings and limitations of this thesis provide areas for future research that can be seen from three different directions. The first direction focuses on further validation of general high-frequency word lists and the ASWL. The second direction is related to the development of different kinds of specialized word lists to better serve the needs of L2 
learners in different contexts. The last direction looks beyond word list construction and validation and calls for more research on different aspects of academic spoken vocabulary.

\subsubsection{Further validation of general-high frequency word lists and the ASWL}

One direction for additional research is to further validate the general high-frequency word lists and the ASWL. The investigation of teacher perception of word usefulness and learner vocabulary knowledge in the present research was purely quantitative. Follow-up studies using a qualitative approach may provide further insights into the validity of general highfrequency word lists (Schmitt, 2010). For example, interviews with a sample of learners and teachers may provide deeper information about learner vocabulary knowledge, and why teachers thought a word useful. In this way, the researcher could confirm if the words were actually known by learners, and had better understanding of factors affecting teachers' ratings. Similarly, the development of the ASWL in this thesis is only a starting point of developing a pedagogical academic spoken word list for L2 learners. For the ASWL to be widely accepted, learners and teachers should be involved in the word list validation. Given the fact that no academic lists of single word units have been validated in this way, further research is needed.

\subsubsection{Development of other specialized word lists}

A second direction is to develop other kinds of word lists to help L2 learners enhance their comprehension of academic spoken and written English. In this way, learners can choose the list that best suits their specific needs. One possible option is to develop disciplinespecific word lists. Although the ASWL provides fairly similar coverage in the four disciplinary sub-corpora, it is slightly more advantageous for applied-subject learners. As suggested in the previous chapter, depending on a certain context, either a universal academic word list or a discipline-specific word list may be a more effective learning tool. Therefore, further research on developing word lists for each disciplinary group would be valuable (Hyland \& Tse, 2007). These lists may better serve the needs of EAP classes that consist of learners planning to study the same or similar disciplines.

To achieve academic success, learners need to have a good knowledge of both academic spoken and written vocabulary. This thesis focuses on academic spoken vocabulary because, while many sources are available for students to improve their knowledge of academic written vocabulary, academic spoken vocabulary resources are very limited. 
However, it is also beneficial to develop an academic written word list (AWWL) along the same lines the ASWL. The consistency in word selection of the ASWL and AWWL will then allow a more valid comparison between the most frequent, wide ranging, and evenly distributed items in academic spoken and written discourse. This will then provide more insight into the differences and similarities in the nature of academic vocabulary in two kinds of academic discourse and better serve EAP learning and teaching.

Moreover, considering the limitations of Juilland and Chang-Rodrigues's (1964) D as a measure of dispersion, the development of these word lists should make use of a more sensitive measure of dispersion.

\subsubsection{Focusing on other aspects of academic spoken vocabulary}

A third option is to look beyond word list construction and validation and examine other features of vocabulary in academic spoken English.

To begin with, this study considered lectures, seminars, labs, and tutorials as a whole in the analysis because it aimed to identify the vocabulary that L2 learners are likely to encounter often in a wide range of speech events. However, because different speech events have distinctive linguistic features (Biber, 2006; Schmitt, Cobb, Horst, \& Schmitt, 2015), further research that examines the lexical demands of each speech event is warranted. Moreover, the ASWL focuses on four classroom speech events (lectures, seminars, labs, and tutorials) to address the primary need of the majority of EAP learners; that is, being able to comprehend and engage in academic courses with their instructors and fellow students. However, the vocabulary in other kinds of speech events (e.g., supervision, workshops for staff, conference presentations, and office hours) deserves attention in further research.

Additionally, the present thesis focuses on single words. However, given the importance of multi-word units (Coxhead, 2006; Simpson-Vlach \& Ellis, 2010), more research is needed in this area. For example, research could look at which words commonly collocate with the ASWL words and to what extend recurring patterns of lexical chunk occur across subject areas. This would provide a useful move from focusing on single word units to multi-word units to help learners expand their knowledge of ASWL words.

Also, this thesis does not look at the influence of polysemy, homonyms, and cognates on the comprehension of general high-frequency words and academic words. Polysemy was 
not the focus of the present study because none of the four general high-frequency word lists deal with this. Moreover, learners' knowledge of polysemy grows incrementally (Crossley, Salsbury, \& McNamara, 2010), and word lists should be seen as a guide rather than a handbook for learners and teachers to follow (Nation, 2016). Wang and Nation (2004) found that the AWL words do not have many meanings. Similar results were found by Parent (2012) with the GSL words. However, the present study shows that vocabulary in academic spoken English is distinctive from that in academic written English, and the BNC/COCA2000 is the most suitable general high-frequency word list for L2 learners. Therefore, an an area that needs further research is whether Wang and Nation's (2004) and Parent's (2012) findings can be still applied in the case of the ASWL and the BNC/COCA2000.

\subsection{Conclusion}

The present research has shed light on the nature of vocabulary in academic spoken English. Importantly, it developed an ASWL that offers benefits to EAP learners irrespective of their disciplines and academic majors, as well as suggesting that Nation's (2012) BNC/COCA2000 is the most suitable general high-frequency word lists for L2 learners. These lists are valuable resources to set learning goals, and select and design materials to support L2 learners' continual vocabulary development. Also, this thesis makes valuable contributions to corpus-based word list construction and validation. Additionally, it provides a fundamental basis for further research into academic spoken vocabulary and word list studies. 


\section{References}

Adolphs, S., \& Carter, R. (2013). Spoken corpus linguistics: From monomodal to multimodal. New York: Routledge.

Aguilar, M. (2016). Seminars. In K. Hyland \& P. Shaw (Eds.), The Routledge handbook of English for Academic Purposes (pp. 335-347). London: Routledge.

Aitchison, J. (1994). Words in the mind: An introduction to the mental lexicon (2nd ed.). Oxford: Blackwell.

Anderson, R. C., \& Freebody, P. (1983). Reading comprehension and the assessment and acquisition of word knowledge. Advances in Reading Language Research, 2, 231256.

Anthony, L. (n.d.). AntwordProfiler. Retrieved from http://www.laurenceanthony.net/antwordprofiler_index.html

Baker, P., Hardie, A., \& McEnery, T. (2006). A glossary of corpus linguistics. Edinburgh: Edinburgh University Press.

Banister, C. (2016). The Academic Word List: Exploring teacher practices, attitudes and beliefs through a web-based survey and interviews. The Journal of Teaching English for Specific and Academic Purposes, 4(2), 309-325.

Barnard, H. (1961). A test of P. U. C students' vocabulary in Chotanagpur. Bulletin of the Central Institute of English, 1, 90-100.

Basturkmen, H. (2003). Specificity and ESP course design. RELC Journal, 34(1), 48-63.

Basturkmen, H. (2006). Ideas and options in English for specific purposes. Mahwah: Lawrence Erlbaum Associates.

Basturkmen, H. (2010). Developing courses in English for Specific Purposes. Houndmills: Palgrave Macmillan. 
Basturkmen, H., \& Shackleford, N. (2015). How content lecturers help students with language: An observational study of language-related episodes in interaction in first year accounting classrooms. English for Specific Purposes, 37, 87-97.

Bauer, L., \& Nation, P. (1993). Word families. International Journal of Lexicography, 6(4), 253-279.

Becher, T. (1989). Academic tribes and territories. Bristol: The Society for Research into Higher Education and Open University Press.

Becher, T., \& Trowler, P. R. (2001). Academic tribes and territories (2nd ed.). Philadephia: Open University Press.

Becker, A. (2016). L2 students' performance on listening comprehension items targeting local and global information. Journal of English for Academic Purposes, 24, 1-13.

Beeckmans, R., Eyckmans, J., Janssens, V., Dufranne, M., \& Van de Velde, H. (2001). Examining the Yes/No vocabulary test: Some methodological issues in theory and practice. Language Testing, 18(3), 235-274.

Biber, D. (1988). Variation across speech and writing. Cambridge: Cambridge University Press.

Biber, D. (1993). Representativeness in corpus design. Literary and Linguistic Computing, $8(4), 243-257$.

Biber, D. (2006). University language: A corpus-based study of spoken and written registers. Amsterdam: John Benjamins.

Biber, D., Conrad, S., Reppen, R., Byrd, P., \& Helt, M. (2002). Speaking and writing in the university: A multidimensional comparison. TESOL Quarterly, 36(1), 9-48.

Biber, D., Conrad, S., Reppen, R., Byrd, P., Helt, M., Clark, V., Cortes, V., Csomay, E., \& Urzua, A. (2004). Representing language use in the university: Analysis of the 
TOEFL 2000 spoken and written academic language corpus. Princeton:

Educational Testing Service.

Biber, D., Johansson, S., Leech, G., Conrad, S., \& Finegan, E. (1999). Longman grammar of spoken and written English. London: Longman.

Biber, D., Reppen, R., Schnur, E., \& Ghanem, R. (2016). On the (non)utility of Juilland's D to measure lexical dispersion in large corpora. International Journal of Corpus Linguistics, 21(4), 439-464.

Biglan, A. (1973a). Relationships between subject matter characteristics and the structure and output of university departments. Journal of Applied Psychology, 57(3), 204213.

Biglan, A. (1973b). The characteristics of subject matter in different academic areas. Journal of Applied Psychology, 57(3), 195-203.

Blue, G. (1988). Individualising academic writing tuition. In P. C. Robinson (Ed.), Academic writing: Process and product (pp. 95-99). Basingstoke: Modern English Publications in association with the British Council.

Bonk, M. (2000). Second language lexical knowledge and listening comprehension. International Journal of Listening, 14(1), 14-31.

Borg, S. (2003). Teacher cognition in language teaching: A review of research on what language teachers think, know, believe, and do. Language Teaching, 36(2), 81-109.

Borg, S. (2006). Teacher cognition and language education. London: Continuum.

Borg, S. (2015). Language teacher cognition bibliography. Retrieved from http://www.education.leeds.ac.uk/people/academic/borg Braine, G. (1999). Introduction. In G. Braine (Ed.), Non-native educators in English language teaching (pp. xiii-xxi). Mahwah: Lawrence Erlbaum. 
Brezina, V. (2014). Statistics Toolbox. Retrieved from http://corpora.lancs.ac.uk/stats/toolbox.php

Brezina, V., \& Gablasova, D. (2015). Is there a core general vocabulary? Introducing the New General Service List. Applied Linguistics, 36(1), 1-22.

Brown, D. (2013). Types of words identified as unknown by L2 learners when reading. System, 41(4), 1043-1055.

Brown, J. D., \& Rodgers, T. S. (2002). Doing second language research. Oxford: Oxford University Press.

Browne, C. (2014). A New General Service List: The better mousetrap we've been looking for? Vocabulary Learning and Instruction, 3(1), 1-10.

Browne, C., Culligan, B., \& Phillips, J. (n.d.). A new academic word list. Retrieved from http://www.newacademicwordlist.org/

Brutten, S. R. (1981). An analysis of student and teacher indications of vocabulary difficulty. RELC Journal, 12(1), 66-71.

Brysbaert, M., \& New, B. (2009). Moving beyond Kučera and Francis: A critical evaluation of current word frequency norms and the introduction of a new and improved word frequency measure for American English. Behavior Research Methods, 41(4), 977-990.

Brysbaert, M., Stevens, M., Mandera, P., \& Keuleers, E. (2016). How many words do we know? Practical estimates of vocabulary size dependent on word definition, the degree of language input and the participant's age. Frontiers in Psychology. https://doi.org/0.3389/fpsyg.2016.01116

Cameron, L. (2002). Measuring vocabulary size in English as an additional language. Language Teaching Research, 6(2), 145-173. 
Campion, M. E., \& Elley, W. B. (1971). An academic vocabulary list. Wellington: New Zealand Council for Educational Research.

Carroll, J. B., Davies, P., \& Richman, B. (1971). The American heritage word frequency book. New York: Houghton Mifflin, Boston American Heritage.

Carter, R. (1987). Vocabulary: Applied linguistic perspectives. Boston: Allen \& Unwin.

Carter, R., \& McCarthy, M. (1988). Vocabulary and language teaching. London: Longman.

Chang, A. C.-S. (2007). The impact of vocabulary preparation on L2 listening comprehension, confidence and strategy use. System, 35(4), 534-550.

Chung, T. M., \& Nation, P. (2004). Identifying technical vocabulary. System, 32(2), 251263.

Cobb, T. (2010). Learning about language and learners from computer programs. Reading in a Foreign Language, 22(1), 181-200.

Cobb, T. (n.d.). Lextutor. Retrieved from http://www.lextutor.ca

Cobb, T., \& Horst, M. (2004). Is there room for an academic word list in French?. In P. Bogaards \& B. Laufer (Eds.), Vocabulary in a second language: Selection, acquisition, and testing (pp. 15-38). Amsterdam: John Benjamins.

Cohen, J. (1988). Statistical power analysis for the behavioral sciences (2nd ed.). Hillsdale: Lawrence Erlbaum Associates.

Corson, D. (1997). The learning and use of academic English words. Language Learning, 47(4), 671-718.

Coxhead, A. (1998). The development and evaluation of an academic word list (Unpublished MA thesis). Victoria University of Wellington, Wellington, New Zealand. 
Coxhead, A. (2000). A new academic word list. TESOL Quarterly, 34(2), 213-238.

Coxhead, A. (2006). Essentials of teaching academic vocabulary. Boston: Heinle, Cengage Learning.

Coxhead, A. (2008). Phraseology and English for Academic Purposes: Challenges and opportunities. In F. Meunier \& S. Granger (Eds.), Phraseology in foreign language learning and teaching (pp. 149-161). Amsterdam: John Benjamins.

Coxhead, A. (2011). The Academic Word List 10 years on: Research and teaching implications. TESOL Quarterly, 45(2), 355-362.

Coxhead, A. (2012). Academic vocabulary, writing and English for Academic Purposes: Perspectives from second language learners. RELC Journal, 43(1), 137-145.

Coxhead, A. (2014). New ways in teaching vocabulary. Alexandria: TESOL Inc.

Coxhead, A. (2016). Reflecting on Coxhead (2000), “A New Academic Word List". TESOL Quarterly, 50(1), 181-185.

Coxhead, A., \& Hirsh, D. (2007). A pilot science-specific word list. Revue Française de Linguistique Appliqueé, 12(2), 65-78.

Coxhead, A. J., \& Walls, R. (2012). TED Talks, vocabulary, and listening for EAP. TESOLANZ Journal, 20, 55-67.

Coxhead, A., Stevens, L., \& Tinkle, J. (2010). Why might secondary science textbooks be difficult to read? New Zealand Studies in Applied Linguistics, 16(2), 37-52.

Crawford Camiciottoli, B., \& Querol-Julián, M. (2016). Lectures. In K. Hyland \& P. Shaw (Eds.), The Routledge handbook of English for Academic Purposes (pp. 309-322). London: Routledge. 
Crossley, S., Salsbury, T., \& McNamara, D. (2010). The development of polysemy and frequency use in English Second Language speakers. Language Learning, 60(3), 573-605.

Csomay, E. (2006). Academic talk in American university classrooms: Crossing the boundaries of oral-literate discourse? Journal of English for Academic Purposes, $5(2), 117-135$.

Daller, H., Milton, J., \& Treffers-Daller, J. (2007). Modelling and assessing vocabulary knowledge. Cambridge: Cambridge University Press.

Dang, T. N. Y., \& Webb, S. (2014). The lexical profile of academic spoken English. English for Specific Purposes, 33, 66-76.

Dang, T. N. Y., \& Webb, S. (2016). Making an essential word list. In I. S. P. Nation (Ed.), Making and using word lists for language learning and testing (pp. 153-167). Amsterdam: John Benjamins.

Davies, M., \& Gardner, D. (2010). A frequency dictionary of contemporary American English: Word sketches, collocates, and thematic lists. New York: Routledge.

Deroey, K. L. B., \& Taverniers, M. (2011). A corpus-based study of lecture functions. Moderna Språk, 2, 2-22.

Dobinson, T. (2001). Do learners learn from classroom interaction and does the teacher have a role to play? Language Teaching Research, 5(3), 189-211.

Dörnyei, Z., \& Taguchi, T. (2010). Questionnaires in second language research. New York: Routledge.

Durrant, P. (2014). Discipline and level specificity in university students' written vocabulary. Applied Linguistics, 35(3), 328-356.

Durrant, P. (2016). To what extent is the Academic Vocabulary List relevant to university student writing? English for Specific Purposes, 43, 49-61. 
Ellis, L. (2002). Teaching from experience: A new perspective on the non-native teacher in adult ESL. Australian Review of Applied Linguistics, 25(1), 71-107.

Ellis, N. C. (2002a). Frequency effects in language processing. Studies in Second Language Acquisition, 24(2), 143-188.

Ellis, N. C. (2002b). Reflections on frequency effects in language processing. Studies in Second Language Acquisition, 24(2), 297-339.

Ellis, N. C., Simpson-Vlach, R., \& Maynard, C. (2008). Formulaic language in native and second language speakers: Psycholinguistics, corpus linguistics, and TESOL. TESOL Quarterly, 42(3), 375-396.

Engels, L. K. (1968). The fallacy of word counts. International Review of Applied Linguistics in Language Teaching, 6(3), 213-231.

Eslami, Z. R., \& Eslami-Rasekh, A. (2007). Discourse markers in academic lectures. Asian EFL Journal, 9(1), 22-38.

Eyckmans, J. (2004). Measuring receptive vocabulary size. Utrecht: Landelijke Onderzoekschool Taalwetenschap.

Fairclough, M. (2011). Testing the lexical recognition task with Spanish/English bilinguals in the United States. Language Testing, 28(2), 273-297.

Farrell, T. S. C. (2015). Language teacher professional development. Alexandria: TESOL Inc.

Flowerdew, J. (1993). Concordancing as a tool in course design. System, 21(2), 231-244.

Flowerdew, J., \& Miller, L. (1992). Student perceptions, problems and strategies in second language lecture comprehension. RELC Journal, 23(2), 60-80.

Francis, W. N., \& Kučera, H. (1982). Frequency analysis of English usage: Lexicon and grammar. Boston: Houghton Mifflin. 
Fung, L., \& Carter, R. (2007). Discourse markers and spoken English: Native and learner use in pedagogic settings. Applied Linguistics, 28(3), 410-439.

Gao, X., \& Ma, Q. (2011). Vocabulary learning and teaching beliefs of pre-service and inservice teachers in Hong Kong and mainland China. Language Awareness, 20(4), $327-342$.

Gardner, D. (2007). Validating the construct of word in applied corpus-based vocabulary research: A critical survey. Applied Linguistics, 28(2), 241-265.

Gardner, D. (2013). Exploring vocabulary: Language in action. New York: Routledge.

Gardner, D., \& Davies, M. (2014). A new academic vocabulary list. Applied Linguistics, 35(3), 305-327.

Gerami, R. G., \& Noordin, N. B. T. (2013). Teacher cognition in foreign language vocabulary teaching: A study of Iranian high school EFL teachers. Theory and Practice in Language Studies, 3(9), 1531-1545.

Ghadessy, P. (1979). Frequency counts, words lists, and materials preparation: A new approach. English Teaching Forum, 17(1), 24-27.

Gilner, L., \& Morales, F. (2008). Corpus-based frequency profiling: Migration to a word list based on the British National Corpus. The Buckingham Journal of Language and Linguistics, 1, 41-58.

Goh, C. C. M. (1999). How much do learners know about the factors that influence their listening comprehension? Hong Kong Journal of Applied Linguistics, 4(1), 17-41.

Goh, C. C. M. (2000). A cognitive perspective on language learners' listening comprehension problems. System, 28(1), 55-75.

Grabe, W. (2009). Reading in a second language: Moving from theory to practice. New York: Cambridge University Press. 
Guo, S. (2011). Impact of an out-of-class activity on students' English awareness, vocabulary, and autonomy. Language Education in Asia, 2(2), 246-256.

Harrington, M., \& Carey, M. (2009). The on-line Yes/No test as a placement tool. System, $37(4), 614-626$.

Harwood, N., \& Petríc, B. (2011). English for Academic Purposes. In J. Simpson (Ed.), The Routledge handbook of Applied Linguistics (pp. 243-258). London: Routledge.

Hasan, A. S. (2000). Learners' perceptions of listening comprehension problems. Language, Culture and Curriculum, 13(2), 137-153.

Henriksen, B., \& Danelund, L. (2015). Studies of Danish L2 learners' vocabulary knowledge and the lexical richness of their written production in English. In P. Pietilä, K. Doró, \& R. Pipalová (Eds.), Lexical issues in L2 writing (pp. 1-27). Newcastle upon Tyne: Cambridge Scholars Publishing.

Hernández, M., Costa, A., \& Arnon, I. (2016). More than words: Multiword frequency effects in non-native speakers. Language, Cognition and Neuroscience, 31(6), 785800.

Hincks, R. (2003). Pronouncing the Academic Word List: Features of L2 student oral presentations. In M. J. Solé, D. Recasens, \& J. Romero (Eds.), Proceedings of the 15th International Congress of Phonetics Sciences (pp. 1545-1548). Barcelona, Spain. Retrieved from http://www.speech.kth.se/ctt/publications/papers03/icphs03_1545.pdf

Hirsh, D., \& Nation, P. (1992). What vocabulary size is needed to read unsimplified texts for pleasure? Reading in a Foreign Language, 8(2), 689-696.

Hoang, V. V. (2010). The current situation and issues of the teaching of English in Vietnam. 立命館言語文化研究, 22(1), 7-18. 
Hsu, W. (2009). College English textbooks for general purposes: A corpus-based analysis of lexical coverage. Electronic Journal of Foreign Language Teaching, 6(1), 42-62.

Hsu, W. (2013). Bridging the vocabulary gap for EFL medical undergraduates: The establishment of a medical word list. Language Teaching Research, 17(4), 454484.

Hsu, W. (2014). Measuring the vocabulary load of engineering textbooks for EFL undergraduates. English for Specific Purposes, 33, 54-65.

Hu, M., \& Nation, I. S. P. (2000). Vocabulary density and reading comprehension. Reading in a Foreign Language, 23, 403-430.

Hwang, K. (1989). Reading newspapers for the improvement of vocabulary and reading skill (Unpublished PhD thesis). Victoria University of Wellington, Wellington, New Zealand.

Hyland, K. (2000). Disciplinary discourses: Social interactions in academic writing. Harlow: Pearson Education Limited.

Hyland, K. (2004). Disciplinary discourses: Social interactions in academic writing. Michigan: University of Michigan Press.

Hyland, K. (2006). English for Academic Purposes: An advanced resource book. London: Routledge.

Hyland, K. (2016). General and specific EAP. In K. Hyland \& P. Shaw (Eds.), The Routledge handbook of English for Academic Purposes (pp. 17-29). London: Routledge.

Hyland, K., \& Shaw, P. (2016). Introduction. In K. Hyland \& P. Shaw (Eds.), The Routledge handbook of English for Academic Purposes (pp. 1-13). London: Routledge.

Hyland, K., \& Tse, P. (2007). Is there an “academic vocabulary”? TESOL Quarterly, 41(2), $235-253$. 
Ippolito, J., Steele, J. L., \& Samson, J. F. (2008). Introduction: Why adolescent literacy matters now. Harvard Educational Review, 78(1), 1-6.

Ivanič, R. (2004). Discourses of writing and learning to write. Language and Education, $18(3), 220-245$.

Jacobs, V. A. (2008). Adolescent literacy: Putting the crisis in context. Harvard Educational Review, 78(1), 7-39.

Jenkins, J. (2009). English as a lingua franca: Interpretations and attitudes. World Englishes, 28(2), 200-207.

Joe, A. G. (2006). The nature of encounters with vocabulary and long-term vocabulary acquisition (Unpublished PhD thesis). Victoria University of Wellington, Wellington, New Zealand.

Jones, W. A. (2011). Variation among academic disciplines: An update on analytical frameworks and research. The Journal of the Professoriate, 6(1), 9-27.

Juilland, A. G., \& Chang-Rodríguez, E. (1964). Frequency dictionary of Spanish words. London: Mouton.

Khani, R., \& Tazik, K. (2013). Towards the development of an academic word list for applied linguistics research articles. RELC Journal, 44(2), 209-232.

Kobeleva, P. P. (2012). Second language listening and unfamiliar proper names: Comprehension barrier? RELC Journal, 43(1), 83-98.

Kolb, D. A. (1981). Learning styles and disciplinary differences. In A. W. Chickering (Ed.), The modern American college (pp. 232-255). San Francisco: Jossey Bass.

Konstantakis, N. (2007). Creating a business word list for teaching business English. Elia, 7, 79-102.

Kwary, D. A. (2011). A hybrid method for determining technical vocabulary. System, 39(2), 175-185. 
Lau, C., \& Rao, N. (2013). English vocabulary instruction in six early childhood classrooms in Hong Kong. Early Child Development and Care, 183(10), 13631380.

Laufer, B. (1989). What percentage of text-lexis is essential for comprehension? In C. Laurén \& M. Nordman (Eds.), Special language: From humans thinking to thinking machines (pp. 316-323). Clevedon: Multilingual Matters.

Laufer, B. (1997). What's in a word that makes it hard or easy: Some intralexical factors that affect the learning of words. In N. Schmitt \& R. Carter (Eds.), Vocabulary: Description, acquisition and pedagogy (pp. 140-155). Cambridge: Cambridge University Press.

Laufer, B. (1998). The development of passive and active vocabulary in a second language: Same or different? Applied Linguistics, 19(2), 255-271.

Laufer, B., \& Hulstijn, J. (2001). Incidental vocabulary acquisition in a second language: The construct of task-induced involvement. Applied Linguistics, 22(1), 1-26.

Laufer, B., \& Nation, P. (1999). A vocabulary-size test of controlled productive ability. Language Testing, 16(1), 33-51.

Laufer, B., \& Ravenhorst-Kalovski, G. C. (2010). Lexical threshold revisited: Lexical text coverage, learners' vocabulary size and reading comprehension. Reading in a Foreign Language, 22(1), 15-30.

Leech, G. N., Rayson, P., \& Wilson, A. (2001). Word frequencies in written and spoken English. Harlow: Longman.

Leeke, P., \& Shaw, P. (2000). Learners' independent records of vocabulary. System, 28(2), $271-289$. 
Lei, L., \& Liu, D. (2016). A new medical academic word list: A corpus-based study with enhanced methodology. Journal of English for Academic Purposes, 22, 42-53.

Lesaux, N. K., Kieffer, M. J., Faller, S. E., \& Kelley, J. G. (2010). The effectiveness and ease of implementation of an academic vocabulary intervention for linguistically diverse students in urban middle schools. Reading Research Quarterly, 45(2), 196228.

Li, Y., \& Qian, D. D. (2010). Profiling the Academic Word List (AWL) in a financial corpus. System, 38(3), 402-411.

Liu, J., \& Han, L. (2015). A corpus-based environmental academic word list building and its validity test. English for Specific Purposes, 39, 1-11.

Lynch, T. (2011). Academic listening in the 21st century: Reviewing a decade of research. Journal of English for Academic Purposes, 10(2), 79-88.

Lynn, R. W. (1973). Preparing word-lists: A suggested method. RELC Journal, 4(1), 2528.

Ma, L. P. F. (2012). Strengths and weaknesses of NESTs and NNESTs: Perceptions of NNESTs in Hong Kong. Linguistics and Education, 23(1), 1-15.

Macalister, J. (2012). Pre-service teacher cognition and vocabulary teaching. RELC Journal, 43(1), 99-111.

MacDonald, M., Badger, R., \& White, G. (2000). The real thing?: Authenticity and academic listening. English for Specific Purposes, 19(3), 253-67.

Martínez, I. A., Beck, S. C., \& Panza, C. B. (2009). Academic vocabulary in agriculture research articles: A corpus-based study. English for Specific Purposes, 28(3), 183198. 
Matsuoka, W., \& Hirsh, D. (2010). Vocabulary learning through reading: Does an ELT course book provide good opportunities? Reading in a Foreign Language, 22(1), 56-70.

Matthews, J., \& Cheng, J. (2015). Recognition of high frequency words from speech as a predictor of L2 listening comprehension. System, 52, 1-13.

McCarthy, M. (1990). Vocabulary. Oxford: Oxford University Press.

McCarthy, M., \& Carter, R. (1997). Written and spoken vocabulary. In N. Schmitt \& M. McCarthy (Eds.), Vocabulary: Description, acquisition and pedagogy (pp. 20-39). Cambridge: Cambridge University Press.

McCarthy, M., \& O’Dell, F. (2008). Academic vocabulary in use. Cambridge: Cambridge University Press.

McLean, S., Kramer, B., \& Beglar, D. (2015). The creation and validation of a listening vocabulary levels test. Language Teaching Research, 19(6), 741-760.

McNeill, A. (2005). Non-native speaker teachers and awareness of lexical difficulty in pedagogical texts. In E. Llurda (Ed.), Non-native language teachers: Perceptions, challenges and contributions to the profession (Vol. 5, pp. 107-128). New York: Springer.

Meara, P. (1992). EFL vocabulary tests. Swansea: Centre for Applied Language Studies.

Meara, P. (1996). The dimensions of lexical competence. In G. Brown, K. Malmkjaer, \& J. Williams (Eds.), Performance and competence in second language acquisition (pp. 33-53). Cambridge: Cambridge University Press.

Meara, P., \& Buxton, B. (1987). An alternative to multiple choice vocabulary tests. Language Testing, 4(2), 142-154. 
Meara, P., \& Jones, G. (1988). Vocabulary size as a placement indicator. In P. Grunwell (Ed.), Applied linguistics in society. London: CILT.

Meara, P., \& Jones, G. (1990). Eurocentres vocabulary size test, version 10KA. Zurich: Eurocentres Learning Service.

Medgyes, P. (1992). Native or non-native: Who's worth more? ELT Journal, 46(4), 340349.

Medgyes, P. (1994). The non-native teacher. London: Macmillan.

Miller, G. A. (1999). On knowing a word. Annual Review of Psychology, 50(1), 1-19.

Milton, J. (2007). Lexical profiles, learning styles and the contruct validity of lexical size tests. In D. Helmut, J. Milton, \& J. Treffers-Daller (Eds.), Modelling and assessing vocabulary knowledge (pp. 47-58). Cambridge: Cambridge University Press.

Milton, J. (2009). Measuring second language vocabulary acquisition. Bristol: Multilingual Matters.

Milton, J., \& Hopkins, N. (2006). Comparing phonological and orthographic vocabulary size: Do vocabulary tests underestimate the knowledge of some learners. The Canadian Modern Language Review, 63(1), 127-147.

Milton, J., Wade, J., \& Hopkins, N. (2010). Aural word recognition and oral competence in English as a foreign language. In R. Chacón-Beltrán, C. Abello-Contesse, \& M. Torreblanca-López (Eds.), Insights into non-native vocabulary teaching and learning (pp. 83-98). Bristol: Multilingual Matters.

Mochida, K., \& Harrington, M. (2006). The Yes/No test as a measure of receptive vocabulary knowledge. Language Testing, 23(1), 73-98.

Mochizuki, M., \& Aizawa, K. (2000). An affix acquisition order for EFL learners: An exploratory study. System, 28(2), 291-304. 
Moir, J. (1996). Task awareness and learning effectiveness: A case study of ten learners' perceptions of a vocabulary learning task (Unpublished MA thesis). Victoria University of Wellington, Wellington, New Zealand.

Morris, L., \& Cobb, T. (2004). Vocabulary profiles as predictors of the academic performance of Teaching English as a Second Language trainees. System, 32(1), 75-87.

Mudraya, O. (2006). Engineering English: A lexical frequency instructional model. English for Specific Purposes, 25(2), 235-256.

Mulligan, D., \& Kirkpatrick, A. (2000). How much do they understand? Lectures, students and comprehension. Higher Education Research \& Development, 19(3), 311-335.

Mungra, P., \& Canziani, T. (2013). Lexicographic studies in medicine: Academic Word List for clinical case histories. Ibérica, 25, 39-62.

Murphy, J., \& Kandil, M. (2004). Word-level stress patterns in the academic word list. System, 32(1), 61-74.

Nagy, W., \& Townsend, D. (2012). Words as tools: Learning academic vocabulary as language acquisition. Reading Research Quarterly, 47(1), 91-108.

Nation, I. S. P. (1990). Teaching and learning vocabulary. New York: Newbury House.

Nation, I. S. P. (2001). How many high frequency words are there in English. Language, Learning and Literature: Studies Presented to Hakan Ringbom. English Department Publications, 4, 167-181.

Nation, I. S. P. (2006). How large a vocabulary is needed for reading and listening? Canadian Modern Language Review, 63(1), 59-82.

Nation, I. S. P. (2007). The four strands. Innovation in Language Learning and Teaching, 1(1), 1-12. 
Nation, I. S. P. (2012). The BNC/COCA word family lists. Retrieved from http://www.victoria.ac.nz/lals/about/staff/paul-nation

Nation, I. S. P. (2013). Learning vocabulary in another language (2nd ed.). Cambridge: Cambridge University Press.

Nation, I. S. P. (2016). Making and using word lists for language learning and testing. Amsterdam: John Benjamins.

Nation, I. S. P., \& Beglar, D. (2007). A vocabulary size test. The Language Teacher, 31(7), 9-13.

Nation, I. S. P., Heatley, A., \& Coxhead, A. (2002). Range: A program for the analysis of vocabulary in texts. Retrieved from http://www.vuw.ac.nz/lals/staff/paul-nation/ nation.aspx

Nation, I. S. P., \& Waring, R. (1997). Vocabulary size, text coverage, and word lists. In N. Schmitt \& McCarthy, M. (Eds.), Vocabulary: Description, acquisition and pedagogy (pp. 6-19). Cambridge: Cambridge University Press.

Nation, I. S. P., \& Webb, S. (2011). Researching and analyzing vocabulary. Boston: Heinle, Cengage Learning.

Nation, P. (1983). Testing and teaching vocabulary. Guidelines, 5(1), 12-25.

Nation, P. (2004). A study of the most frequent word families in the British National Corpus. In P. Bogaards \& B. Laufer (Eds.), Vocabulary in a second language: Selection, acquisition, and testing (pp. 3-14). Amsterdam: John Benjamins.

Nation, P. (2012). Teaching communicative and interactive vocabulary for EFL learners. In H. P. Widodo \& A. Cirocki (Eds.), Innovation and creativity in ELT methodology (pp. 135-144). New York: Nova Science Publishers. 
Nation, P., \& Coxhead, A. (2014). Vocabulary size research at Victoria University of Wellington, New Zealand. Language Teaching, 47(3), 398-403.

Nation, P., \& Hwang, K. (1995). Where would general service vocabulary stop and special purposes vocabulary begin? System, 23(1), 35-41.

Nation, P., \& Yamamoto, A. (2012). Applying the four strands to language learning. International Journal of Innovation in English Language Teaching and Research, 1(2), 167-181.

Nesi, H. (2002). An English Spoken Academic Word List. In A. Braasch \& C. Povlsen (Eds.), Proceedings of the Tenth EURALEX International Congress (Vol. 1, pp. 351-358). Copenhagen, Denmark. Retrieved from http://www.euralex.org/elx_proceedings/Euralex2002/036_2002_V1_Hilary\%20Ne si_An\%20English\%20Spoken\%20Academic\%20Wordlist.pdf

Nesi, H., \& Basturkmen, H. (2006). Lexical bundles and discourse signaling in academic lecturers. International Journal of Corpus Linguistics, 11(3), 283-304.

Neufeld, S., Hancioğlu, N., \& Eldridge, J. (2011). Beware the range in RANGE, and the academic in AWL. System, 39(4), 533-538.

Neumann, R. (2001). Disciplinary differences and university teaching. Studies in Higher Education, 26(2), 135-146.

Nguyen, T. M. H., \& Webb, S. (2016). Examining second language receptive knowledge of collocation and factors that affect learning. Language Teaching Research, 1 -23. https://doi.org/10.1177/1362168816639619

Nural, S. (2012). EAP teachers' cognitions and practices in teaching lexis in two Turkish private universities: An exploratory qualitative study (Unpublished $\mathrm{PhD}$ thesis). University of Leicester, Leicester.

Nurweni, A., \& Read, J. (1999). The English vocabulary knowledge of Indonesian university students. English for Specific Purposes, 18(2), 161-175.

O’Keeffe, A., McCarthy, M., \& Carter, R. (2007). From corpus to classroom: Language use and language teaching. Cambridge: Cambridge University Press. 
Parent, K. (2012). The most frequent English homonyms. RELC Journal, 43(1), 69-81.

Paribakht, T. S., \& Webb, S. (2016). The relationship between academic vocabulary coverage and scores on a standardized English proficiency test. Journal of English for Academic Purposes, 21, 121-132.

Pellicer-Sánchez, A., \& Schmitt, N. (2012). Scoring Yes-No vocabulary tests: Reaction time vs. nonword approaches. Language Testing, 29(4), 489-509.

Pinchbeck, G. G. (2014, March). Lexical frequency profiling of a large sample of Canadian high school diploma exam expository writing: L1 and L2 academic English. Paper presented at the Roundtable presentation at American Association of Applied Linguistics, Portland, OR, USA.

Praninskas, J. (1972). American university word list. London: Longman.

Qian, D. D. (1999). Assessing the roles of depth and breadth of vocabulary knowledge in reading comprehension. Canadian Modern Language Review, 56(2), 282-307.

Quero, B. (2015). Estimating the vocabulary size of L1 Spanish ESP learners and the vocabulary load of medical textbooks (Unpublished $\mathrm{PhD}$ thesis). Victoria University of Wellington, Wellington, New Zealand.

Quinn, G. (1968). The English vocabulary of some Indonesian university entrants. Salatiga: English Department Monograph IKIP Kristen Satya Watjana.

Rahimi, S. (2014). Teacher cognition vis-à-vis vocabulary teaching. Theory and Practice in Language Studies, 4(3), 652-660.

Read, J. (2000). Assessing vocabulary. Cambridge: Cambridge University Press.

Richards, J. C. (1974). Word lists: Problems and prospects. RELC Journal, 5(2), 69-84.

Richards, J. C. (1976). The role of vocabulary teaching. TESOL Quarterly, 10(1), 77-89.

Richards, J. C. (1983). Listening comprehension: Approach, design, procedure. TESOL Quarterly, 17(2), 219-240. 
Richards, J. C. (2010). Competence and performance in language teaching. RELC Journal, 41(2), 101-122.

Richards, J. C., \& Farrell, T. S. C. (2005). Professional development for language teachers: Strategies for teacher learning. Cambridge: Cambridge University Press.

Rodgers, M. P. H. (2013). English language learning through viewing television: An investigation of comprehension, incidental vocabulary acquisition, lexical coverage, attitudes, and captions (Unpublished $\mathrm{PhD}$ thesis). Victoria University of Wellington, Wellington, New Zealand.

Rodgers, M. P. H., \& Webb, S. (2011). Narrow viewing: The vocabulary in related television programs. TESOL Quarterly, 45(4), 689-717.

Santos, T. (1988). Professors' reactions to the academic writing of nonnative-speaking students. TESOL Quarterly, 22(1), 69-90.

Saville-Troike, M. (1984). What really matters in second language learning for academic achievement? TESOL Quarterly, 18(2), 199-219.

Schmitt, D. (2016, September). Beyond caveat emptor: Applying validity criteria to word lists. Paper presented at the Vocab@Tokyo, Tokyo, Japan.

Schmitt, N. (2000). Vocabulary in language teaching. Cambridge: Cambridge University Press.

Schmitt, N. (2008). Review article: Instructed second language vocabulary learning. Language Teaching Research, 12(3), 329-363.

Schmitt, N. (2010). Researching vocabulary: A vocabulary research manual. New York: Palgrave Macmillan. 
Schmitt, N., Cobb, T., Horst, M., \& Schmitt, D. (2015). How much vocabulary is needed to use English? Replication of van Zeeland \& Schmitt (2012), Nation (2006) and Cobb (2007). Language Teaching. https://doi.org/10.1017/S0261444815000075

Schmitt, N., Jiang, X., \& Grabe, W. (2011). The percentage of words known in a text and reading comprehension. The Modern Language Journal, 95(1), 26-43.

Schmitt, N., \& Meara, P. (1997). Research vocabulary through a word knowledge framework. Studies in Second Language Acquisition, 19(1), 17-36.

Schmitt, N., \& Schmitt, D. (2014). A reassessment of frequency and vocabulary size in L2 vocabulary teaching. Language Teaching, 47(4), 484-503.

Schmitt, N., Schmitt, D., \& Clapham, C. (2001). Developing and exploring the behaviour of two new versions of the Vocabulary Levels Test. Language Testing, 18(1), 5588.

Schmitt, N., \& Zimmerman, C. B. (2002). Derivative word forms: What do learners know? TESOL Quarterly, 36(2), 145-171.

Shillaw, J. (1996). The application of Rasch modelling to Yes/No vocabulary tests. Retrieved from http://www.lognostics.co.uk/

Simpson-Vlach, R., \& Ellis, N. C. (2010). An Academic Formulas List: New methods in phraseology research. Applied Linguistics, 31(4), 487-512.

Sinclair, J. (1991). Corpus, concordance, collocation. Oxford: Oxford University Press. Sinclair, J. (1987). Collins COBUILD English language dictionary. London: Collins.

Smith, C., \& Tajino, A. (2003). Teachers' perceptions of learner difficulty in vocabulary learning. Retrieved from http://www.developingteachers.com/articles_tchtraining/tchvocabpf_craigakira.htm 
Sorell, C. J. (2013). A study of issues and techniques for creating core vocabulary lists for English as an international language (Unpublished $\mathrm{PhD}$ thesis). Victoria University of Wellington, Wellington, New Zealand.

Stæhr, L. S. (2008). Vocabulary size and the skills of listening, reading and writing. The Language Learning Journal, 36(2), 139-152.

Stæhr, L. S. (2009). Vocabulary knowledge and advanced listening comprehension in English as a foreign language. Studies in Second Language Acquisition, 31(4), 577607.

Stubbe, R. (2012). Do pseudoword false alarm rates and overestimation rates in Yes/No vocabulary tests change with Japanese university students' English ability levels? Language Testing, 29(4), 471-488.

Sutarsyah, C., Nation, P., \& Kennedy, G. (1994). How useful is EAP vocabulary for ESP? A corpus based case study. RELC Journal, 25(2), 34-50.

Swan, M., \& Smith, B. (2001). Learner English: A teacher's guide to interference and other problems. Cambridge: Cambridge University Press.

Thompson, P. (2006). A corpus perspective on the lexis of lectures, with a focus on economics lectures. In K. Hyland \& M. Bondi (Eds.), Academic discourse across disciplines (pp. 253-270). New York: Peter Lang.

Thorndike, E. L. (1921). The teacher's word book. New York: Teachers College Columbia University.

Thorndike, E. L., \& Lorge, I. (1944). The teacher' word book of 30,000 words. New York: Bureau of Publications, Teacher College, Columbia University.

Todd, R. W. (2017). An opaque engineering word list: Which words should a teacher focus on? English for Specific Purposes, 45, 31-39. 
Ton, N. N. H., \& Pham, H. H. (2010). Vietnamese teachers' and students' perceptions of global English. Language Education in Asia, 1(1), 48-61.

Townsend, D., \& Collins, P. (2009). Academic vocabulary and middle school English learners: An intervention study. Reading and Writing, 22(9), 993-1019.

Townsend, D., Filippini, A., Collins, \& Biancarosa, G. (2012). Evidence for the importance of academic word knowledge for the academic achievement of diverse middle school students. The Elementary School Journal, 112(3), 497-518.

Valipouri, L., \& Nassaji, H. (2013). A corpus-based study of academic vocabulary in chemistry research articles. Journal of English for Academic Purposes, 12(4), 248263.

van Zeeland, H., \& Schmitt, N. (2013). Lexical coverage in L1 and L2 listening comprehension: The same or different from reading comprehension? Applied Linguistics, 34(4), 457-479.

Vandergrift, L. (2006). Second language listening: Listening ability or language proficiency? Modern Language Journal, 90(1), 6-18.

Vidal, K. (2003). Academic listening: A source of vocabulary acquisition? Applied Linguistics, 24(1), 56-89.

Vidal, K. (2011). A comparison of the effects of reading and listening on incidental vocabulary acquisition. Language Learning, 61(1), 219-258.

Wang, J., Liang, S., \& Ge, G. (2008). Establishment of a Medical Academic Word List. English for Specific Purposes, 27(4), 442-458.

Wang, M. -t. ., \& Nation, P. (2004). Word meaning in academic English: Homography in the Academic Word List. Applied Linguistics, 25(3), 291-314.

Ward, J. (1999). How large a vocabulary do EAP engineering students need? Reading in a Foreign Language, 12(2), 309-323. 
Ward, J. (2009). A basic engineering English word list for less proficient foundation engineering undergraduates. English for Specific Purposes, 28(3), 170-182.

Ward, J., \& Chuenjundaeng, J. (2009). Suffix knowledge: Acquisition and applications. System, 37(3), 461-469.

Webb, S. (2009a). The effects of receptive and productive learning of word pairs on vocabulary knowledge. RELC Journal, 40(3), 360-376.

Webb, S. A. (2009b). The effects of pre-learning vocabulary on reading comprehension and writing. The Canadian Modern Language Review, 65(3), 441-470.

Webb, S. A., \& Chang, A. C.-S. (2012). Second language vocabulary growth. RELC Journal, 43(1), 113-126.

Webb, S. A., \& Sasao, Y. (2013). New directions in vocabulary testing. RELC Journal, 44(3), 263-277.

Webb, S., \& Macalister, J. (2013). Is text written for children useful for L2 extensive reading? TESOL Quarterly, 47(2), 300-322.

Webb, S., \& Nation, P. (2008). Evaluating the vocabulary load of written text. TESOLANZ Journal, 16, 1-10.

Webb, S., \& Paribakht, T. S. (2015). What is the relationship between the lexical profile of test items and performance on a standardized English proficiency test? English for Specific Purposes, 38, 34-43.

Webb, S., \& Rodgers, M. P. H. (2009a). The lexical coverage of movies. Applied Linguistics, 30(3), 407-427.

Webb, S., \& Rodgers, M. P. H. (2009b). Vocabulary demands of television programs. Language Learning, 59(2), 335-366.

Webb, S., Sasao, Y., \& Ballance, O. (n.d.). New Vocabulary Levels Test. Retrieved from at http://vuw.qualtrics.com/jfe/form/SV_4MG1wByukg1JoTb 
West, M. (1953). A general service list of English words. London: Longman, Green.

Worthington, D., \& Nation, P. (1996). Using texts to sequence the introduction of new vocabulary in an EAP course. RELC Journal, 27(2), 1-11.

Xing, P., \& Fulcher, G. (2007). Reliability assessment for two versions of Vocabulary Levels Tests. System, 35(2), 182-191.

Xue, G., \& Nation, P. (1984). A university word list. Language Learning and Communication, 3(2), 215-229.

Yang, M.-N. (2015). A nursing academic word list. English for Specific Purposes, 37, 2738.

Zhang, W. (2008). In search of English as a foreign language (EFL) teachers' knowledge of vocabulary instruction (Unpublished $\mathrm{PhD}$ thesis). Georgia State University, Atlanta.

Zimmerman, C. B. (2005). Teachers' perceptions of lexical anomalies: A pilot study. In P. Bruthiaux, D. Atkinson, W. G. Eggington, W. Grabe, \& V. Ramanathan (Eds.), Directions in applied linguistics: Essays in honor of Robert Kaplan (pp. 131-145). Clevedon: Multilingual Matters.

Zipf, G. (1949). Human behavior and the principle of least effort: An introduction to human ecology. New York: Hafner.

Zipf, G. K. (1935). The psycho-biology of language. Cambridge: MIT Press. 


\section{Appendix 1. Sample of the learner Yes/ No tests}

Trước khi làm bài Test, xin vui lòng điền một số thông tin sau đây:

Tên:

Lớp:

Số năm học tiếng Anh từ trước tới nay:

\section{TEST 1}

Với mỗi từ dưới đây, khoanh tròn: "Yes" nếu bạn biết ít nhất 1 nghĩa của từ "No" nếu bạn không chắc từ đó nghĩa là gì

Lưu ý, một số từ trong bài test không tồn tại trong tiếng Anh. Vì vậy xin bạn hãy thành thật khi đưa ra câu trả lời.

Ví dụ, nếu bạn biết ił nhất 1 nghĩa của tù "go", nhung không biết nghĩa củatìn "hapgood", câu trả lời của bạn sẽ nhu sau:
1. go
(Yes) No
2. hapgood
Yes No

\begin{tabular}{|c|c|c|c|c|c|c|c|c|c|c|}
\hline 1. & angel & Yes & No & 16. & circumsemilar & Yes & No & 31. & awake & Yes \\
\hline 2. & garrisotte & Yes & No & 17. & dictalate & Yes & No & 32. & alongside & Yes \\
\hline 3. & approve & Yes & No & 18. & baptistal & Yes & No & 33. & worse & Yes \\
\hline 4. & britnell & Yes & No & 19. & error & Yes & No & 34. & dirty & Yes \\
\hline 5. & cell & Yes & No & 20. & medium & Yes & No & 35. & attunative & Yes \\
\hline 6. & lamb & Yes & No & 21. & emerge & Yes & No & 36. & passenger & Yes \\
\hline 7. & shortly & Yes & No & 22. & isolate & Yes & No & 37. & exhaust & Yes \\
\hline 8. & hedge & Yes & No & 23. & response & Yes & No & 38. & excuse & Yes \\
\hline 9. & urban & Yes & No & 24. & online & Yes & No & 39. & disaddle & Yes \\
\hline 10. & pine & Yes & No & 25. & handy & Yes & No & 40. & outline & Yes \\
\hline 11. & majority & Yes & No & 26. & towel & Yes & No & 41. & protein & Yes \\
\hline 12. & lid & Yes & No & 27. & yandle & Yes & No & 42. & arrest & Yes \\
\hline 13. & draconite & Yes & No & 28. & boush & Yes & No & 43. & rob & Yes \\
\hline 14. & knife & Yes & No & 29. & accurate & Yes & No & 44. & tongue & Yes \\
\hline 15. & reckon & Yes & No & 30. & shell & Yes & No & 45. & complicate & Yes \\
\hline
\end{tabular}

Xem tiếp ở trang bên $\rightarrow$ 


\section{Appendix 1. Sample of the learner Yes/ No tests (Cont.)}

\begin{tabular}{|c|c|c|c|c|c|c|c|c|c|c|}
\hline 46. & weird & Yes & No & 65. & comprise & Yes & No & 84. & refuge & Yes \\
\hline 47. & mollet & Yes & No & 66. & display & Yes & No & 85. & investebrate & Yes \\
\hline 48. & evaculate & Yes & No & 67. & crisp & Yes & No & 86. & linnard & Yes \\
\hline 49. & layer & Yes & No & 68. & column & Yes & No & 87. & climaximal & Yes \\
\hline 50. & oak & Yes & No & 69. & eldred & Yes & No & 88. & pardoe & Yes \\
\hline 51. & bomb & Yes & No & 70. & smash & Yes & No & 89. & assess & Yes \\
\hline 52. & detailoring & Yes & No & 71. & regime & Yes & No & 90. & kit & Yes \\
\hline 53. & gammonary & Yes & No & 72. & pretend & Yes & No & 91. & leap & Yes \\
\hline 54. & hobby & Yes & No & 73. & colliver & Yes & No & 92. & paralogue & Yes \\
\hline 55. & deliction & Yes & No & 74. & episode & Yes & No & 93. & pub & Yes \\
\hline 56. & pat & Yes & No & 75. & amphlett & Yes & No & 94. & shy & Yes \\
\hline 57. & boost & Yes & No & 76. & alright & Yes & No & 95. & essential & Yes \\
\hline 58. & amey & Yes & No & 77. & recenticle & Yes & No & 96. & prince & Yes \\
\hline 59. & grass & Yes & No & 78. & slave & Yes & No & 97. & core & Yes \\
\hline 60. & homoglyph & Yes & No & 79. & classinate & Yes & No & & & \\
\hline 61. & hobrow & Yes & No & 80. & wool & Yes & No & & & \\
\hline 62. & finalism & Yes & No & 81. & ventrice & Yes & No & & & \\
\hline 63. & troake & Yes & No & 82. & dormatize & Yes & No & & & \\
\hline 64. & navy & Yes & No & 83. & category & Yes & No & & & \\
\hline
\end{tabular}




\section{Appendix 2. Pseudowords in Study 2}

\begin{tabular}{|c|c|c|c|c|c|c|}
\hline abandonate & 36 & apricoterie & 71 & benevolate & 106 & chlorosate \\
\hline abanical & 37 & apsitis & 72 & berrow & 107 & choreostat \\
\hline abhorrify & 38 & arain & 73 & blisterial & 108 & churchlow \\
\hline ablute & 39 & arbus & 74 & bodelate & 109 & circumsemilar \\
\hline abrogative & 40 & arrate & 75 & boobier & 110 & clarination \\
\hline absolvention & 41 & arroster & 76 & boush & 111 & classinate \\
\hline abundal & 42 & artigan & 77 & brandrick & 112 & claypole \\
\hline accomplition & 43 & ashill & 78 & braquette & 113 & climaximal \\
\hline ackery & 44 & ashment & 79 & brimble & 114 & coath \\
\hline ackrill & 45 & aspection & 80 & britnell & 115 & coincidate \\
\hline acquince & 46 & assar & 81 & bulbicate & 116 & collexis \\
\hline actie & 47 & asslam & 82 & bundock & 117 & colliver \\
\hline adamic & 48 & assuasion & 83 & burse & 118 & coloniate \\
\hline adlamate & 49 & astell & 84 & buttle & 119 & combustulate \\
\hline agrinomy & 50 & astin & 85 & cadaverate & 120 & compilate \\
\hline ainge & 51 & atribus & 86 & cadle & 121 & composture \\
\hline aistrope & 52 & attard & 87 & callisthemia & 122 & concannon \\
\hline albucolic & 53 & atter & 88 & cambule & 123 & condick \\
\hline algoric & 54 & attunative & 89 & canarify & 124 & condimented \\
\hline allam & 55 & auner & 90 & candish & 125 & condron \\
\hline allaway & 56 & ballotage & 91 & cantileen & 126 & congulate \\
\hline allegal & 57 & banderage & 92 & captivise & 127 & conseverate \\
\hline almanical & 58 & baptistal & 93 & cardination & 128 & constagnate \\
\hline amagran & 59 & barette & 94 & carotic & 129 & contortal \\
\hline amelicant & 60 & bargery & 95 & cartledge & 130 & contramand \\
\hline amey & 61 & barmion & 96 & casling & 131 & contrivial \\
\hline amiel & 62 & barnden & 97 & casquinade & 132 & convolition \\
\hline amphlett & 63 & barnish & 98 & catalypso & 133 & copner \\
\hline ancett & 64 & barrate & 99 & causticate & 134 & cordonize \\
\hline ancrum & 65 & bastionate & 100 & centipath & 135 & cornock \\
\hline andow & 66 & batcock & 101 & certical & 136 & correctivate \\
\hline antecodal & 67 & batteric & 102 & challinor & 137 & cotargent \\
\hline antile & 68 & beament & 103 & charactal & 138 & cowdery \\
\hline aposterical & 69 & beautitude & 104 & cheatle & 139 & cranicle \\
\hline appertonal & 70 & bechelet & 105 & chicorate & 140 & crayonal \\
\hline
\end{tabular}




\section{Appendix 2. Pseudowords in Study 2 (continued)}

\begin{tabular}{|c|c|c|c|c|c|c|c|}
\hline 141 & crean & 176 & dormatize & 211 & flamboyment & 246 & horozone \\
\hline 142 & crevicing & 177 & dowrick & 212 & floralate & 247 & hospite \\
\hline 143 & crole & 178 & draconite & 213 & fluctual & 248 & hoult \\
\hline 144 & crucialate & 179 & dring & 214 & fragrality & 249 & hudd \\
\hline 145 & cundy & 180 & dubiate & 215 & frake & 250 & humberoid \\
\hline 146 & cunnion & 181 & dumbrill & 216 & frequid & 251 & hypodemical \\
\hline 147 & curify & 182 & durative & 217 & froment & 252 & hyppolite \\
\hline 148 & customate & 183 & dyslaxative & 218 & fruital & 253 & immagical \\
\hline 149 & cymballic & 184 & ebullible & 219 & fumicant & 254 & impelirous \\
\hline 150 & damnifest & 185 & effectory & 220 & galeology & 255 & incarminate \\
\hline 151 & darch & 186 & elative & 221 & gamage & 256 & incorpulent \\
\hline 152 & darrock & 187 & eldred & 222 & gammonary & 257 & inertible \\
\hline 153 & decaphage & 188 & elode & 223 & garrisotte & 258 & infernalise \\
\hline 154 & defunctionary & 189 & elphick & 224 & gasson & 259 & ingredible \\
\hline 155 & degate & 190 & eluctant & 225 & gazard & 260 & insertate \\
\hline 156 & deliction & 191 & emissive & 226 & genderation & 261 & instere \\
\hline 157 & demaine & 192 & encopulate & 227 & gillen & 262 & integrality \\
\hline 158 & demoronic & 193 & enigmanic & 228 & glandle & 263 & interisation \\
\hline 159 & descript & 194 & enormatize & 229 & gravology & 264 & intuned \\
\hline 160 & detailoring & 195 & enviate & 230 & griffing & 265 & investebrate \\
\hline 161 & devigorous & 196 & equalic & 231 & guardie & 266 & ionopose \\
\hline 162 & devoidance & 197 & evaculate & 232 & gumm & 267 & jemmett \\
\hline 163 & dewbery & 198 & eventualise & 233 & haime & 268 & jerram \\
\hline 164 & dictalate & 199 & expedalize & 234 & hamp & 269 & jotham \\
\hline 165 & diggle & 200 & expostulant & 235 & hapgood & 270 & jubicle \\
\hline 166 & dildorous & 201 & extance & 236 & haque & 271 & justal \\
\hline 167 & diplosaur & 202 & extravagate & 237 & harmonical & 272 & keable \\
\hline 168 & disaddle & 203 & fabriculate & 238 & hawther & 273 & kearle \\
\hline 169 & discordage & 204 & factile & 239 & hegedoxy & 274 & kerkin \\
\hline 170 & disinfantal & 205 & faminisation & 240 & hermantic & 275 & kiley \\
\hline 171 & disportal & 206 & fancett & 241 & hierograph & 276 & kitely \\
\hline 172 & distantial & 207 & fastitude & 242 & hignall & 277 & laborative \\
\hline 173 & diversal & 208 & feery & 243 & hobrow & 278 & lamble \\
\hline 174 & documentate & 209 & filterite & 244 & homoglyph & 279 & laminastic \\
\hline 175 & dogmatile & 210 & finalism & 245 & horobin & 280 & lannery \\
\hline
\end{tabular}




\section{Appendix 2. Pseudowords in Study 2 (continued)}

\begin{tabular}{|c|c|c|c|c|c|c|c|}
\hline 281 & lanworn & 316 & matsell & 351 & pallot & 386 & prelatoriat \\
\hline 282 & lapidoscope & 317 & mealing & 352 & panolepsy & 387 & presential \\
\hline 283 & laudalize & 318 & methodontist & 353 & paralogue & 388 & presuppository \\
\hline 284 & leaity & 319 & miligrate & 354 & pardoe & 389 & prickett \\
\hline 285 & lediard & 320 & minestory & 355 & participline & 390 & primality \\
\hline 286 & legender & 321 & mingay & 356 & patronistic & 391 & pring \\
\hline 287 & legitimal & 322 & minshell & 357 & pauling & 392 & proctalize \\
\hline 288 & leopradate & 323 & misabrogate & 358 & peakman & 393 & professive \\
\hline 289 & libidinize & 324 & mollet & 359 & pegler & 394 & propend \\
\hline 290 & limbrick & 325 & mordue & 360 & pellow & 395 & proscratify \\
\hline 291 & limidate & 326 & morphew & 361 & pendulate & 396 & provisual \\
\hline 292 & linnard & 327 & mortalage & 362 & perceptacle & 397 & prowt \\
\hline 293 & linocat & 328 & motivize & 363 & peritonic & 398 & pruden \\
\hline 294 & litholect & 329 & moule & 364 & pernicate & 399 & psychodactic \\
\hline 295 & litotal & 330 & mourant & 365 & pestulant & 400 & pungid \\
\hline 296 & liverick & 331 & mudge & 366 & phonicate & 401 & putbrace \\
\hline 297 & loaring & 332 & multiplify & 367 & picardine & 402 & puttock \\
\hline 298 & logalation & 333 & murtagh & 368 & piccolotomy & 403 & pyrographic \\
\hline 299 & logam & 334 & mynott & 369 & pilbean & 404 & quorant \\
\hline 300 & lorey & 335 & nebulate & 370 & pillotherapy & 405 & ralling \\
\hline 301 & loveridge & 336 & negalogue & 371 & pimlott & 406 & randle \\
\hline 302 & lunarous & 337 & neutration & 372 & pinkard & 407 & recenticle \\
\hline 303 & luque & 338 & nichee & 373 & pither & 408 & remonic \\
\hline 304 & mabbitt & 339 & nickling & 374 & pitten & 409 & renigrade \\
\hline 305 & mabey & 340 & nonagrate & 375 & placorate & 410 & repreach \\
\hline 306 & majury & 341 & noot & 376 & plebocrat & 411 & reservory \\
\hline 307 & malignify & 342 & norval & 377 & podiast & 412 & restificate \\
\hline 308 & maltass & 343 & oaten & 378 & polythetic & 413 & restructive \\
\hline 309 & manolect & 344 & obliquate & 379 & pornucopia & 414 & retrogradient \\
\hline 310 & manomize & 345 & oestrogeny & 380 & portingale & 415 & rhind \\
\hline 311 & marjorize & 346 & opinarchy & 381 & possumate & 416 & rhoden \\
\hline 312 & martlew & 347 & ordinisation & 382 & posteriorate & 417 & richings \\
\hline 313 & mascarate & 348 & oxylate & 383 & postherent & 418 & rickard \\
\hline 314 & mastiphitis & 349 & pagatherm & 384 & pragmadict & 419 & ridiculate \\
\hline 315 & mateer & 350 & paladine & 385 & preconagulative & 420 & roscrow \\
\hline
\end{tabular}




\section{Appendix 2. Pseudowords in Study 2 (continued)}

\begin{tabular}{|c|c|c|c|}
\hline 421 & rudall & 456 & temerify \\
\hline 422 & sacrumate & 457 & tennick \\
\hline 423 & saliate & 458 & torpedal \\
\hline 424 & samphirate & 459 & treadaway \\
\hline 425 & sandry & 460 & trinder \\
\hline 426 & savourite & 461 & troake \\
\hline 427 & scally & 462 & trudgeon \\
\hline 428 & schismal & 463 & tudball \\
\hline 429 & seclunar & 464 & undery \\
\hline 430 & sedgebeer & 465 & utting \\
\hline 431 & semaphrodite & 466 & varney \\
\hline 432 & seward & 467 & ventrice \\
\hline 433 & shattock & 468 & veronicide \\
\hline 434 & shellard & 469 & waggett \\
\hline 435 & shide & 470 & wallage \\
\hline 436 & shreeve & 471 & warboy \\
\hline 437 & skelding & 472 & watchorn \\
\hline 438 & skemp & 473 & watler \\
\hline 439 & skene & 474 & waygood \\
\hline 440 & sleeth & 475 & weate \\
\hline 441 & sloggett & 476 & wolliner \\
\hline 442 & solitist & 477 & woodarch \\
\hline 443 & sollis & 478 & wookey \\
\hline 444 & spratling & 479 & yallop \\
\hline 445 & sprudd & 480 & yandle \\
\hline 446 & stephonitis & & \\
\hline 447 & stillhard & & \\
\hline 448 & stimulcrate & & \\
\hline 449 & strategory & & \\
\hline 450 & subsistify & & \\
\hline 451 & suddery & & \\
\hline 452 & sumption & & \\
\hline 453 & swannick & & \\
\hline 454 & swithin & & \\
\hline 455 & synott & & \\
\hline
\end{tabular}




\section{Appendix 3. Ethics approval}

TE WHARE WX̃NANGA O TE OPOKO O TE IKA A MĀUI

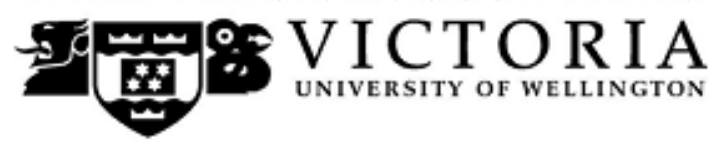

MEMORANDUM

Phone $\quad 0-4-4635676$

Fax 0-4-4635209

Email Allison.kirkman@vuw.ac.nz

\begin{tabular}{l|l}
\hline TO & Thi Ngoc Yen Dang \\
\hline COPY TO & Stuart Webb \\
\hline FROM & Dr Allison Kirkman, Convener, Human Ethics Committee \\
\hline
\end{tabular}

\begin{tabular}{l|l}
\hline DATE & 2 June 2014 \\
\hline PAGES & 1 \\
\hline \multicolumn{2}{|l}{} \\
\hline SUBJECT & $\begin{array}{l}\text { Ethics Approval: } 20951 \\
\text { Developing and validating academic written and spoken word lists }\end{array}$ \\
\hline
\end{tabular}

Thank you for your application for ethical approval, which has now been considered by the Standing Committee of the Human Ethics Committee.

Your application has been approved from the above date and this approval continues until 1 October 2016. If your data collection is not completed by this date you should apply to the Human Ethics Committee for an extension to this approval.

Best wishes with the research.

Allison Kirkman

Human Ethics Committee

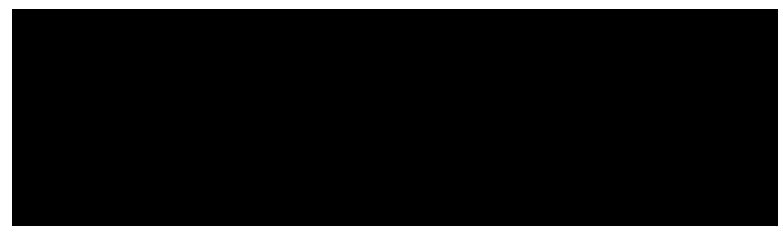




\section{Appendix 4. Amendment approval}

TE WHARE WÃNANGA O TE ÕPOKO O TE IKA A MĀUI

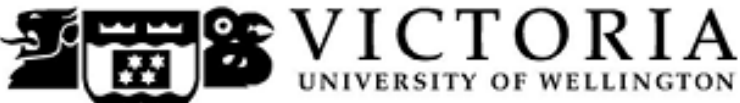

$\begin{array}{lll} & \text { Phone } & 044635676 \\ \text { M E M OR A N D U M } & \text { Fax } & 044653209 \\ \text { Email } & \text { Allisonkirmman@vuwacnz }\end{array}$

\begin{tabular}{l|l}
\hline TO & Thi Ngoc Yen Dang \\
\hline COPY TO & Stuart Webb \\
\hline FROM & Dr Allison Kirkman, Convener, Human Ethics Committee \\
\hline
\end{tabular}

\begin{tabular}{l|l}
\hline DATE & 30 January 2015 \\
\hline PAGES & 1 \\
\hline
\end{tabular}

SUBJECT $\quad$ Ethics Approval: 20951

Developing and validating academic written and spoken word lists

Thank you for your request to amend your ethics approval. This has now been considered and the request granted.

Your application has approval until 1 October 2016. If your data collection is not completed by this date you should apply to the Human Ethics Committee for an extension to this approval.

Best wishes with the research.

Allison Kirkman

Human Ethics Committee 


\section{Appendix 5. Teacher information sheet}

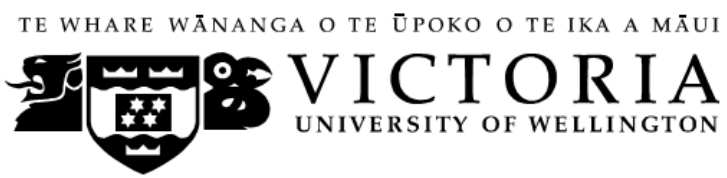

School of Linguistics and Applied Language Studies

TEACHER INFORMATION SHEET

\section{Research title: Developing and validating academic written and spoken word lists Researcher: Thi Ngoc Yen Dang, PhD student}

I would like to invite you to take part in research which is trying to find which English words are known by second language (L2) learners. It has been approved by the Human Ethics Committee of Victoria University of Wellington, New Zealand.

In this study, you will do 10 short surveys in which you will indicate the usefulness of the English words to L2 learners in a 5-band Likert scale. Each survey takes around 5-10 minutes to complete. You can do these surveys in your free time and send them back to me by email when you have finished. This research will help to identify the most useful words for teaching to second language learners. This should have great pedagogical value. For your participation, you will be emailed information on the research findings and the benefits of the research relating to language learning and teaching, on request. You will also be given a small gift to thank for your participation in this study.

Your participation in this study is voluntary. You are free to decline or withdraw from the study any time without giving any reasons. If you would like to withdraw from the study, just let me know by sending me an email before the next survey is conducted.Your responses to all tasks will be confidential. All data collected for this project will be stored securely so that only the researcher has access to it. The findings from the project will be used in conference presentations and in articles to be submitted to journals in applied linguistics.

This research will help teachers of English such as yourselves know how to teach more effectively and will help learners to learn English more effectively.

Thank you very much for your time. If you have any questions, free to contact either me or my supervisor, Dr. Averil Coxhead via the following address.

\section{Thi Ngoc Yen Dang}

School of Linguistic \& Applied Language Studies Victoria University of Wellington PO Box 600, Wellington 6140, New Zealand

Email: ThiNgocYen.Dang@vuw.ac.nz

\section{Dr. Averil Coxhead}

School of Linguistic \& Applied Language Studies

Victoria University of Wellington

PO Box 600, Wellington 6140, New Zealand

Email: averil.coxhead@vuw.ac.nz

Yours sincerely,

Thi Ngoc Yen Dang 


\section{Appendix 6. Teacher consent form}

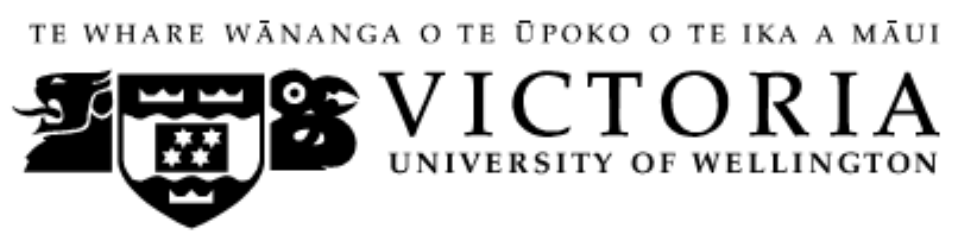

School of Linguistics and Applied Language Studies

\section{CONSENT FORM}

Title: Developing and validating academic written and spoken word lists

\section{Researcher: Thi Ngoc Yen Dang, PhD student}

I have had an opportunity to ask questions and have them answered to my satisfaction.

I also understand that I may withdraw my participation from this project at any stage during data collection.

I agree/do not agree (delete one) to be part of this research study

Signed:

Date:

Name:

Yes, I would like to be sent a summary of results from the research, and my e-mail address is: 


\title{
Appendix 7. Learner information sheet
}

\author{
TE WHARE WĀNANGA O TE ŪPOKO O TE IKA A MĀUI

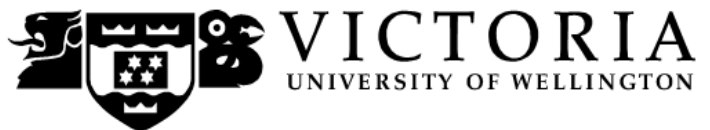 \\ THÔNG TIN VỂ ĐỀ TÀI NGHIÊN CÚU
}

\section{Đề tài: Phát triển và đánh giá danh sách từ vựng trong Tiếng Anh nói và viết học thuật}

Trân thành mời bạn tham gia nghiên cứu khảo sát vốn từ vựng của người học Tiếng Anh như một tiếng nước ngoài. Nghiên cứu được thực hiện bởi Đặng Thị Ngọc Yến, Nghiên cứu sinh ngành Ngôn ngữ học ứng dụng, ĐH Victoria, Wellington, New Zealand Nghiên cứu đã được Hội đồng thẩm định đạo đức trong nghiên cứu của trường ĐH Victoria, Wellington, New Zealand phê duyệt.

Nghiên cứu sẽ được tiến hành trong giờ giải lao hoặc sau giờ học Tiếng Anh của bạn. Bạn sẽ làm 16 bài kiểm tra ngắn trong đó bạn sẽ chỉ ra những từ tiếng Anh mà bạn biết trong bài kiểm tra. Không có giới hạn trong thời gian làm bài kiểm tra. Kết quả các bài kiểm tra chỉ được sự dụng cho mục đích nghiên cứu. Kết quả đó sẽ không được thông báo cho giáo viên Tiếng Anh của bạn và không ảnh hưởng tới kết quả học tập của bạn. Tham gia nghiên cứu này là cơ hội tốt để bạn đánh giá lượng từ vựng của mình. Nếu bạn yêu cầu, sau khi nghiên cứu kết thúc, bạn sẽ nhận được kết quả đánh giá lượng từ của mình, cùng với những lời khuyên hữu ích về phương pháp để nâng cao vốn từ vựng.

Việc bạn tham gia nghiên cứu này hoàn toàn mang tính tự nguyện. Bạn có quyền từ chối, hoặc dừng tham gia mà không cần đưa ra bất cứ lý do nào. Nếu bạn không muốn tiếp tục tham gia nghiên cứu, xin vui lòng gửi email thông báo cho tôi trước khi bài kiểm tra tiếp theo được tiến hành. Nếu bạn không muốn tham gia nghiên cứu này, bạn vẫn dự lớp học và tham gia các hoạt động trong giờ học như bình thường. Nghiên cứu sẽ không ảnh hưởng gì tới điểm số cũng như kết quả học tập của bạn trong khóa học. Mọi dữ liệu từ nghiên cứu sẽ được bảo mật, chỉ có người thực hiện đề tài được tiếp cận những dữ liệu này. Kết quả của nghiên cứu sẽ được công bố ở các hội thảo và tạp chí khoa học chuyên ngành Ngôn ngữ học ứng dụng.

Nghiên cứu này sẽ giúp cho giáo viên Tiếng Anh nâng cao chất lượng giảng dạy và giúp cho sinh viên như bạn học tiếng Anh hiệu quả hơn.

Xin chân thành cảm ơn. Nếu bạn cần thêm bất cứ thông tin gì hoặc có bất cứ thắc mắc nào liên quan tới nghiên cứu, xin vui lòng liên hệ với tôi hoặc giáo viên hướng dẫn của tôi, Tiến sỹ Averil Coxhead theo địa chỉ:

\section{Đặng Thị Ngoc Yến}

School of Linguistic \& Applied Language Studies Victoria University of Wellington

PO Box 600, Wellington 6140, New Zealand

Email: ThiNgocYen.Dang@vuw.ac.nz

\section{Tiến sĩ Averil Coxhead}

School of Linguistic \& Applied Language Studies

Victoria University of Wellington

PO Box 600, Wellington 6140, New Zealand

Email: averil.coxhead@vuw.ac.nz 


\section{Appendix 8. Learner information sheet (English translation)}

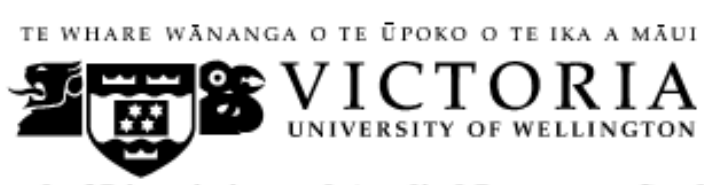

School of Linguistics and Applied Language Studies

STUDENT INFORMATION SHEET

\section{Research title: Developing and validating academic written and spoken word lists Researcher: Thi Ngoc Yen Dang, PhD student}

I would like to invite you to take part in a study that will take place during your English lessons. This research is trying to find which English words are known by second language learners. It has been approved by the Human Ethics Committee of Victoria University of Wellington, New Zealand.

In these lessons you will do 16 short tests in which you will indicate the words that you know. You will have as much time as you need to complete the tests. The tests are only being used for research purposes and your performance on the tests will have no effect on your class grades. Moreover, your teachers will not be given your scores on the tests. These tests will be useful opportunities for you to assess your vocabulary knowledge. For your participation, you will receive your scores with some interpretation including your estimated vocabulary size and tips on how to improve your vocabulary knowledge, on request.

Your participation in this study is voluntary. You are free to decline or withdraw from the study any time without giving any reasons. If you would like to withdraw from the study, just let me know by sending me an email before the next test is conducted. If you decide not to participate in the study you will still attend class as normal and will carry out similar activities as part of your regular classroom programme. The study will have no impact on course grades or on successfully completing the course. Your responses to all tasks will be confidential. All data collected for this project will be stored securely so that only the researcher has access to it. The findings from the project will be used in conference presentations and in articles to be submitted to journals in applied linguistics.

This research will help teachers of English know how to teach more effectively and will help learners such as yourselves to learn English more effectively.

Thank you very much for your time. If you have any questions, free to contact either me or my supervisor, Dr. Averil Coxhead via the following address.

\section{Thi Ngoc Yen Dang}

School of Linguistic \& Applied Language Studies Victoria University of Wellington PO Box 600, Wellington 6140, New Zealand

Email:ThiNgocYen.Dang@vuwac.nz

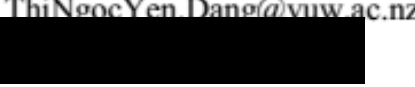

Yours sincerely,

Thi Ngoc Yen Dang

\section{Dr. Averil Coxhead}

School of Linguistic \& Applied Language Studies Victoria University of Wellington

PO Box 600, Wellington 6140, New Zealand

Email: averil.coxhead@vuw.ac.nz 


\title{
Appendix 9. Learner consent form
}

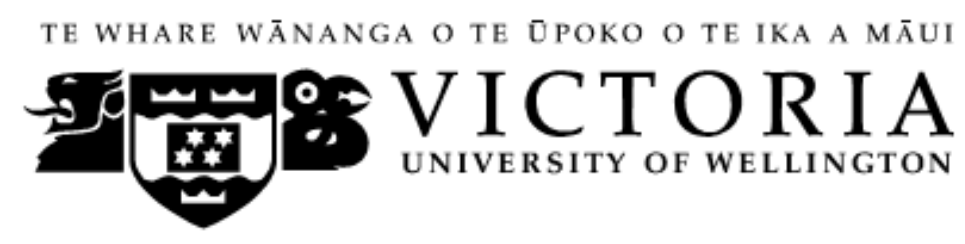

\section{BẢN CHẤP THUẬN THAM GIA NGHIÊN CÚU}

\author{
Đề tài: Phát triển và đánh giá danh sách từ vựng trong tiếng Anh nói và viết học thuật \\ Người nghiên cứu: Đặng Thị Ngọc Yến \\ Nghiên cứu sinh ngành Ngôn ngữ học ứng dụng, ĐH Victoria, Wellington, New Zealand
}

प Tôi đã được người thực hiện đề tài cung cấp và giải thích đầy đủ thông tin liên quan đến đề tài

Tôi biết rằng tôi có quyền dừng tham gia bất cứ lúc nào

Do do, tôi đồng ý/ không đồng ý (Khoanh tròn một lựa chọn) tham gia nghiên cứu

Chữ ký:

Ngày:

Tên:

Có, tôi muốn được thông báo kết quả vốn từ vựng của mình, địa chỉ email của tôi là: 


\section{Appendix 10. Learner consent form (English translation)}

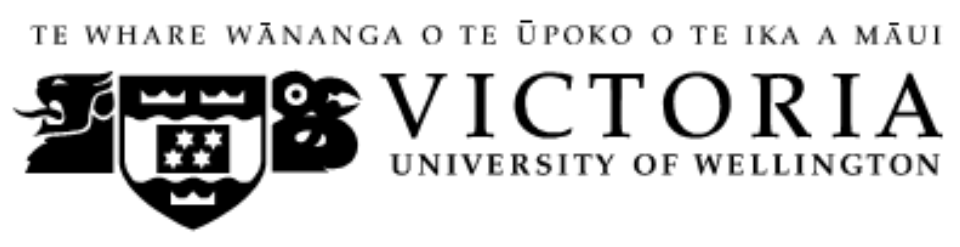

School of Linguistics and Applied Language Studies

\section{CONSENT FORM}

Title: Developing and validating academic written and spoken word lists

\section{Researcher: Thi Ngoc Yen Dang, PhD student}

I have had an opportunity to ask questions and have them answered to my satisfaction.

I also understand that I may withdraw my participation from this project at any stage during data collection.

I agree/do not agree (delete one) to be part of this research study

Signed:

Date:

Name:

Yes, I would like to be sent the result of my vocabulary level, and my e-mail address is: 


\section{Appendix 11. Spoken materials from Yale courses}

\section{African American History}

Holloway, Jonathan. African American History: From Emancipation to the Present, Spring 2010. (Yale University: Open Yale Courses), http://oyc.yale.edu (Accessed 16 Jan, 2015). License: Creative Common BY-NC-SA.

\section{American studies}

Dimock, Wai Chee. Hemingway, Fitzgerald, Faulkner, Fall 2011. (Yale University: Open Yale Courses), http://oyc.yale.edu (Accessed 16 Jan, 2015). License: Creative Common BY-NC-SA.

\section{Astronomy}

Bailyn, Charles. Frontiers and Controversies in Astrophysic, Spring 2007. (Yale University: Open Yale Courses), http://oyc.yale.edu (Accessed 16 Jan, 2015). License: Creative Common BY-NC-SA.

\section{Biomedical Engineering}

Saltzman, W. Mark. Frontiers of Biomedical Engineering, Spring 2008. Yale University: Open Yale Courses), http://oyc.yale.edu (Accessed 16 Jan, 2015). License: Creative Common BY-NC-SA.

\section{Chemistry}

McBride, J. Michael. Freshman Organic Chemistry I, Fall 2008. (Yale University: Open Yale Courses), http://oyc.yale.edu (Accessed 16 Jan, 2015). License: Creative Common BY-NC-SA.

McBride, J. Michael. Freshman Organic Chemistry II, Spring 2011. (Yale University: Open Yale Courses), http://oyc.yale.edu (Accessed 16 Jan, 2015). License: Creative Common BY-NC-SA.

\section{Classic Studies}

Kagan, Donald. Introduction to Ancient Greek History, Fall 2007. (Yale University: Open Yale Courses), http://oyc.yale.edu (Accessed 16 Jan, 2015). License: Creative Common BY-NC-SA.

\section{Ecology and Evolutionary Biology}

Stearns, Stephen C. Principles of Evolution, Ecology and Behavior, Spring 2009. (Yale University: Open Yale Courses), http://oyc.yale.edu (Accessed 16 Jan, 2015). License: Creative Common BY-NC-SA.

\section{Economics}

Geanakoplos, John. Financial Theory, Fall 2009. (Yale University: Open Yale Courses), http://oyc.yale.edu (Accessed 16 Jan, 2015). License: Creative Common BY-NC-SA. 


\section{Appendix 11. Spoken materials from Yale courses (continued)}

Polak, Ben. Game Theory, Fall 2007. (Yale University: Open Yale Courses), http://oyc.yale.edu (Accessed 16 Jan, 2015). License: Creative Common BY-NC-SA.

Shiller, Robert J. Financial Markets, Spring 2008. (Yale University: Open Yale Courses), http://oyc.yale.edu (Accessed 16 Jan, 2015). License: Creative Common BY-NC-SA.

Shiller, Robert J. Financial Markets, Spring 2011. (Yale University: Open Yale Courses), http://oyc.yale.edu (Accessed 16 Jan, 2015). License: Creative Common BY-NC-SA.

\section{Environmental Studies}

Wargo, John. Environmental Politics and Law, Spring 2010. (Yale University: Open Yale Courses), http://oyc.yale.edu (Accessed 16 Jan, 2015). License: Creative Common BY-NCSA.

\section{Geology and Geophysics}

Smith, Ronald B. The Atmosphere, the Ocean, and Environmental Change, Fall 2011. (Yale University: Open Yale Courses), http://oyc.yale.edu (Accessed 16 Jan, 2015). License: Creative Common BY-NC-SA.

\section{History}

Blight, David W. The Civil War and Reconstruction Era, 1845-1877, Spring 2008. (Yale University: Open Yale Courses), http://oyc.yale.edu (Accessed 16 Jan, 2015). License: Creative Common BY-NC-SA.

Freeman, Joanne. The American Revolution, Spring 2010. (Yale University: Open Yale Courses), http://oyc.yale.edu (Accessed 16 Jan, 2015). License: Creative Common BY-NCSA.

Freedman, Paul. The Early Middle Ages, 284-1000. Fall 2011. (Yale University: Open Yale Courses), http://oyc.yale.edu (Accessed 16 Jan, 2015). License: Creative Common BY-NC-SA.

Merriman, John. France Since 1871, Fall 2007. (Yale University: Open Yale Courses), http://oyc.yale.edu (Accessed 16 Jan, 2015). License: Creative Common BY-NC-SA.

Merriman, John. European Civilization, 1648-1945, Fall 2008. (Yale University: Open Yale Courses), http://oyc.yale.edu (Accessed 16 Jan, 2015). License: Creative Common BY-NC-SA.

Snowden, Frank. Epidemics in Western Society Since 1600, Spring 2010. (Yale University: Open Yale Courses), http://oyc.yale.edu (Accessed 16 Jan, 2015). License: Creative Common BY-NC-SA.

Wrightson, Keith E. Early Modern England: Politics, Religion, and Society under the Tudors and Stuarts, Fall 2009. (Yale University: Open Yale Courses), http://oyc.yale.edu (Accessed 16 Jan, 2015). License: Creative Common BY-NC-SA. 


\section{Appendix 11. Spoken materials from Yale courses (continued)}

\section{History of Art}

Kleiner, Diana E. E. Roman Architecture, Spring 2009. (Yale University: Open Yale Courses), http://oyc.yale.edu (Accessed 16 Jan, 2015). License: Creative Common BY-NCSA.

Wyman, Robert. Global Problems of Population Growth, Spring 2009. (Yale University: Open Yale Courses), http://oyc.yale.edu (Accessed 16 Jan, 2015). License: Creative Common BY-NC-SA.

\section{Molecular, Cellular and Developmental Biology}

Wyman, Robert. Global Problems of Population Growth, Spring 2009. (Yale University: Open Yale Courses), http://oyc.yale.edu (Accessed 16 Jan, 2015). License: Creative Common BY-NC-SA.

\section{Philosophy}

Gendler, Tamar. Philosophy and the Science of Human Nature, Spring 2011. (Yale University: Open Yale Courses), http://oyc.yale.edu (Accessed 16 Jan, 2015). License: Creative Common BY-NC-SA.

Kagan, Shelly. Death, Spring 2007. (Yale University: Open Yale Courses), http://oyc.yale.edu (Accessed 16 Jan, 2015). License: Creative Common BY-NC-SA.

\section{Physics}

Shankar, Ramamurti. Fundamentals of Physics I, Fall 2006. (Yale University: Open Yale Courses), http://oyc.yale.edu (Accessed 16 Jan, 2015). License: Creative Common BY-NCSA.

Shankar, Ramamurti. Fundamentals of Physics II, Spring 2010. (Yale University: Open Yale Courses), http://oyc.yale.edu (Accessed 16 Jan, 2015). License: Creative Common BY-NC-SA.

\section{Political Science}

Rae, Douglas W. Capitalism: Success, Crisis, and Reform, Fall 2009. (Yale University: Open Yale Courses), http://oyc.yale.edu (Accessed 16 Jan, 2015). License: Creative Common BY-NC-SA.

Shapiro, Ian. The Moral Foundations of Politics, Spring 2010. (Yale University: Open Yale Courses), http://oyc.yale.edu (Accessed 16 Jan, 2015). License: Creative Common BY-NCSA.

Smith, Steven B. Introduction to Political Philosophy, Fall 2006. (Yale University: Open Yale Courses), http://oyc.yale.edu (Accessed 16 Jan, 2015). License: Creative Common BY-NC-SA. 


\section{Appendix 11. Spoken materials from Yale courses (continued)}

\section{Psychology}

Bloom, Paul. Introduction to Psychology, Spring 2007. (Yale University: Open Yale Courses), http://oyc.yale.edu (Accessed 16 Jan, 2015). License: Creative Common BY-NCSA.

Brownell, Kelly D. The Psychology, Biology and Politics of Food, Fall 2008. Yale University: Open Yale Courses), http://oyc.yale.edu (Accessed 16 Jan, 2015). License: Creative Common BY-NC-SA.

\section{Religious Studies}

Martin, Dale B. Introduction to the New Testament History and Literature, Spring 2009. (Yale University: Open Yale Courses), http://oyc.yale.edu (Accessed 16 Jan, 2015). License: Creative Common BY-NC-SA.

Hayes, Christine. Introduction to the Old Testament (Hebrew Bible), Fall 2006. (Yale University: Open Yale Courses), http://oyc.yale.edu (Accessed 16 Jan, 2015). License: Creative Common BY-NC-SA.

\section{Sociology}

Szelényi, Iván. Foundations of Modern Social Theory, Fall 2009. (Yale University: Open Yale Courses), http://oyc.yale.edu (Accessed 16 Jan, 2015). License: Creative Common BY-NC-SA. 


\section{Appendix 12. Spoken materials from Massachusetts Institute of Technology courses}

\section{Astronomy}

Richard Battin. 16.346 Astrodynamics, Fall 2008. (Massachusetts Institute of Technology: MIT OpenCourseWare), http://ocw.mit.edu (Accessed 14 Jan, 2015). License: Creative Commons BY-NC-SA

\section{Biology}

Claudette Gardel, Eric Lander, Robert Weinberg, and Andrew Chess. 7.012 Introduction to Biology, Fall 2004. (Massachusetts Institute of Technology: MIT OpenCourseWare), http://ocw.mit.edu (Accessed 14 Jan, 2015). License: Creative Commons BY-NC-SA

Eric Lander, Robert Weinberg, Tyler Jacks, Hazel Sive, Graham Walker, Sallie Chisholm, and Michelle Mischke. 7.01SC Fundamentals of Biology, Fall 2011. (Massachusetts Institute of Technology: MIT OpenCourseWare), http://ocw.mit.edu(Accessed 14 Jan, 2015). License: Creative Commons BY-NC-SA

Graham Walker, Julia Khodor, Michelle Mischke, and Penny Chisholm. 7.014 Introductory Biology, Spring 2005. (Massachusetts Institute of Technology: MIT OpenCourseWare), http://ocw.mit.edu (Accessed 14 Jan, 2015). License: Creative Commons BY-NC-SA

Hazel Sive, and Tyler Jacks. 7.013 Introductory Biology, Spring 2006. (Massachusetts Institute of Technology: MIT OpenCourseWare), http://ocw.mit.edu (Accessed 14 Jan, 2015). License: Creative Commons BY-NC-SA

\section{Chemistry}

Catherine Drennan, and Elizabeth Taylor. 5.111 Principles of Chemical Science, Fall 2008. (Massachusetts Institute of Technology: MIT

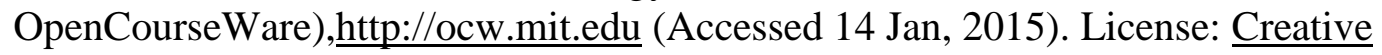
Commons BY-NC-SA

Christopher Cummins, and Sylvia Ceyer. 5.112 Principles of Chemical Science, Fall 2005. (Massachusetts Institute of Technology: MIT OpenCourseWare), $\underline{\text { http://ocw.mit.edu (Accessed } 14 \text { Jan, 2015). License: Creative }}$ Commons BY-NC-SA

Donald Sadoway. 3.091SC Introduction to Solid State Chemistry, Fall 2010. (Massachusetts Institute of Technology: MIT OpenCourseWare), http://ocw.mit.edu(Accessed 14 Jan, 2015). License: $\underline{\text { Creative }}$ Commons BY-NC-SA

\section{Comparative Media Studies}

Philip Tan, and Jason Begy. CMS.608 Game Design, Fall 2010. (Massachusetts Institute of Technology: MIT OpenCourseWare), http://ocw.mit.edu (Accessed 15 Jan, 2015).

License: Creative Commons BY-NC-SA 


\section{Appendix 12. Spoken materials from Massachusetts Institute of Technology courses (continued) \\ Economic}

Esther Duflo, and Abhijit Banerjee. 14.73 The Challenge of World Poverty, Spring 2011. (Massachusetts Institute of Technology: MIT

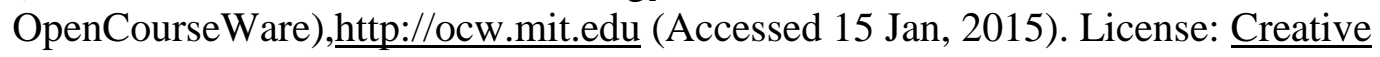
Commons BY-NC-SA

Jonathan Gruber. 14.01SC Principles of Microeconomics, Fall 2011. (Massachusetts Institute of Technology: MIT OpenCourseWare), http://ocw.mit.edu (Accessed 15 Jan, 2015). License: Creative Commons BY-NC-SA

\section{Electrical engineering \& computer science}

Anant Agarwal. 6.002 Circuits and Electronics, Spring 2007. (Massachusetts Institute of Technology: MIT OpenCourseWare), http://ocw.mit.edu (Accessed 15 Jan, 2015). License: Creative Commons BY-NC-SA

Bernhardt Wuensch. 3.60 Symmetry, Structure, and Tensor Properties of Materials, Fall 2005. (Massachusetts Institute of Technology: MIT

OpenCourseWare), http://ocw.mit.edu (Accessed 15 Jan, 2015). License: $\underline{\text { Creative }}$ Commons BY-NC-SA

Eric Grimson, Peter Szolovits, and Trevor Darrell. 6.001 Structure and Interpretation of Computer Programs, Spring 2005. (Massachusetts Institute of Technology: MIT OpenCourseWare), http://ocw.mit.edu (Accessed 15 Jan, 2015). License: Creative Commons BY-NC-SA

Erik Demaine. 6.849 Geometric Folding Algorithms: Linkages, Origami, Polyhedra, Fall 2012. (Massachusetts Institute of Technology: MIT

OpenCourseWare), $\underline{\text { http://ocw.mit.edu (Accessed } 15 \text { Jan, 2015). License: Creative }}$ Commons BY-NC-SA

Erika Wagner. ESD.172J X PRIZE Workshop: Grand Challenges in Energy, Fall 2009. (Massachusetts Institute of Technology: MIT

OpenCourseWare), http://ocw.mit.edu(Accessed 15 Jan, 2015). License: $\underline{\text { Creative }}$ Commons BY-NC-SA

Maxwell Mann. 6.370 The Battlecode Programming Competition, January IAP 2013. (Massachusetts Institute of Technology: MIT OpenCourseWare), http://ocw.mit.edu(Accessed 15 Jan, 2015). License: $\underline{\text { Creative }}$ Commons BY-NC-SA

Robert Gallager. 6.262 Discrete Stochastic Processes, Spring 2011. (Massachusetts Institute of Technology: MIT OpenCourseWare), http://ocw.mit.edu (Accessed 15 Jan, 2015). License: Creative Commons BY-NC-SA

Rodric Rabbah, and Saman Amarasinghe. 6.189 Multicore Programming Primer, January IAP 2007. (Massachusetts Institute of Technology: MIT

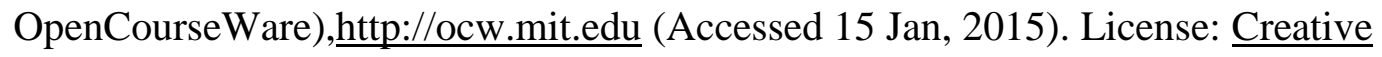
Commons BY-NC-SA 


\section{Appendix 12. Spoken materials from Massachusetts Institute of Technology courses (continued)}

Saman Amarasinghe, and Charles Leiserson. 6.172 Performance Engineering of Software Systems, Fall 2010. (Massachusetts Institute of Technology: MIT

OpenCourseWare), http://ocw.mit.edu (Accessed 15 Jan, 2015). License: Creative

Commons BY-NC-SA

Soheil Feizi-Khankandi, and Muriel Médard. 6.450 Principles of Digital Communication I, Fall 2009. (Massachusetts Institute of Technology: MIT

OpenCourseWare),http://ocw.mit.edu (Accessed 15 Jan, 2015). License: Creative

Commons BY-NC-SA

\section{Law}

Keith Winstein. 6.912 Introduction to Copyright Law, January IAP 2006. (Massachusetts Institute of Technology: MIT OpenCourseWare), http://ocw.mit.edu(Accessed 15 Jan, 2015). License: Creative Commons BY-NC-SA

\section{Management}

John Sterman, and Hazhir Rahmandad. 15.871 Introduction to System Dynamics, Fall 2013. (Massachusetts Institute of Technology: MIT

OpenCourseWare),http://ocw.mit.edu (Accessed 15 Jan, 2015). License: Creative Commons BY-NC-SA

\section{Mathematics}

Arthur Mattuck, Haynes Miller, Jeremy Orloff, and John Lewis. 18.03SC Differential Equations, Fall 2011. (Massachusetts Institute of Technology: MIT OpenCourseWare),http://ocw.mit.edu (Accessed 14 Jan, 2015). License: Creative Commons BY-NC-SA

Charles Leiserson, and Erik Demaine. 6.046J Introduction to Algorithms (SMA 5503), Fall 2005. (Massachusetts Institute of Technology: MIT

OpenCourseWare),http://ocw.mit.edu (Accessed 14 Jan, 2015). License: Creative Commons BY-NC-SA

Denis Auroux. 18.02SC Multivariable Calculus, Fall 2010. (Massachusetts Institute of Technology: MIT OpenCourseWare), http://ocw.mit.edu (Accessed 14 Jan, 2015). License: Creative Commons BY-NC-SA

Gilbert Strang. 18.086 Mathematical Methods for Engineers II, Spring 2006. (Massachusetts Institute of Technology: MIT OpenCourseWare), http://ocw.mit.edu(Accessed 14 Jan, 2015). License: Creative Commons BY-NC-SA

Gilbert Strang. 18.085 Computational Science and Engineering I, Fall 2008. (Massachusetts Institute of Technology: MIT OpenCourseWare), http://ocw.mit.edu(Accessed 14 Jan, 2015). License: $\underline{\text { Creative }}$ Commons BY-NC-SA 


\section{Appendix 12. Spoken materials from Massachusetts Institute of Technology courses (continued)}

Gilbert Strang. 18.06 Linear Algebra, Spring 2010. (Massachusetts Institute of Technology: MIT OpenCourseWare), http://ocw.mit.edu (Accessed 14 Jan, 2015). License: Creative Commons BY-NC-SA

\section{Music \& theatre}

Christopher Ariza. 21M.380 Music and Technology (Contemporary History and Aesthetics), Fall 2009. (Massachusetts Institute of Technology: MIT OpenCourseWare), http://ocw.mit.edu (Accessed 15 Jan, 2015). License: Creative Commons BY-NC-SA

Mark Harvey, and Tom Hall. 21M.355 Musical Improvisation, Spring 2013. (Massachusetts Institute of Technology: MIT

OpenCourseWare), http://ocw.mit.edu(Accessed 15 Jan, 2015). License: $\underline{\text { Creative }}$ Commons BY-NC-SA

Teresa Neff. 21M.250 Beethoven to Mahler, Spring 2014. (Massachusetts Institute of Technology: MIT OpenCourseWare), http://ocw.mit.edu (Accessed 15 Jan, 2015).

License: Creative Commons BY-NC-SA

\section{Physics}

Allan Adams, Matthew Evans, and Barton Zwiebach. 8.04 Quantum Physics I, Spring 2013. (Massachusetts Institute of Technology: MIT

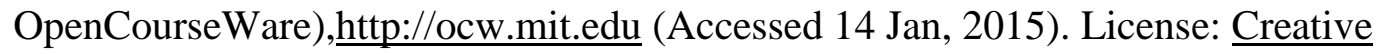
Commons BY-NC-SA

Alan Guth. 8.286 The Early Universe, Fall 2013. (Massachusetts Institute of Technology: MIT OpenCourseWare), http://ocw.mit.edu (Accessed 14 Jan, 2015). License: $\underline{\text { Creative }}$ Commons BY-NC-SA

Mehran Kardar. 8.333 Statistical Mechanics I: Statistical Mechanics of Particles, Fall 2013. (Massachusetts Institute of Technology: MIT

OpenCourseWare), http://ocw.mit.edu (Accessed 14 Jan, 2015). License: $\underline{\text { Creative }}$ Commons BY-NC-SA 


\section{Appendix 13. Spoken materials from University of California, Berkeley}

Art

McNamara, John S. Practice of Art 8, 2014. (University of California, Berkeley: UC Berkeley OpenCourseWare), http://webcast.berkeley.edu (Accessed 18 Jan, 2015). License: Creative Common BY-NC-SA 3.0.

Niemeyer, Greg., \& Hilston, James. Practice of Art 23AC, 2011. (University of California, Berkeley: UC Berkeley OpenCourseWare), http://webcast.berkeley.edu (Accessed 18 Jan, 2015). License: Creative Common BY-NC-SA 3.0.

\section{Astronomy \& Ecology}

Allen, Richard. Earth and Planetary Science C20, 2014. (University of California, Berkeley: UC Berkeley OpenCourseWare),http://webcast.berkeley.edu (Accessed 18 Jan, 2015). License: Creative Common BY-NC-SA 3.0.

Marcy, Geoffrey W. Earth and Planetary Science C12, 2014. (University of California, Berkeley: UC Berkeley OpenCourseWare),http://webcast.berkeley.edu (Accessed 18 Jan, 2015). License: Creative Common BY-NC-SA 3.0.

\section{Bioengineering}

Graves, David B. Chemical \& Biomolecular Engineering 179, 2014. (University of California, Berkeley: UC Berkeley OpenCourseWare),http://webcast.berkeley.edu (Accessed 18 Jan, 2015). License: Creative Common BY-NC-SA 3.0.

Healy, Kevin E. Bioengineering 200, 2015. (University of California, Berkeley: UC Berkeley OpenCourseWare),http://webcast.berkeley.edu (Accessed 18 Jan, 2015). License: Creative Common BY-NC-SA 3.0.

\section{Chemistry}

Stacy, Angelica. Chemistry 1A, 2012. (University of California, Berkeley: UC Berkeley OpenCourseWare), http://webcast.berkeley.edu (Accessed 18 Jan, 2015). License: Creative Common BY-NC-SA 3.0.

\section{Civil engineering}

Panagiotou, Marios Agathoklis. Civil and Environmental Engineering 120, 2012.(University of California, Berkeley: UC Berkeley OpenCourseWare),http://webcast.berkeley.edu (Accessed 18 Jan, 2015). License: Creative Common BY-NC-SA 3.0.

\section{Culture studies}

Cohen Michael. American Studies 10AC,2012 (University of California, Berkeley: UC Berkeley OpenCourseWare), http://webcast.berkeley.edu (Accessed 18 Jan, 2015). License: Creative Common BY-NC-SA 3.0. 


\section{Appendix 13. Spoken materials from University of California, Berkeley (continued)}

Wallace, John R. East Asian Languages and Cultures 109, 2014. (University of California, Berkeley: UC Berkeley OpenCourseWare),http://webcast.berkeley.edu (Accessed 18 Jan, 2015). License: Creative Common BY-NC-SA 3.0.

\section{Geography}

Sayre, Nathan F. Geography 130, 2015.(University of California, Berkeley: UC Berkeley OpenCourseWare),http://webcast.berkeley.edu (Accessed 10 March, 2015). License: Creative Common BY-NC-SA 3.0.

\section{Journalism}

Drummond, William, J. Journalism 102AC, 2012. (University of California, Berkeley: UC Berkeley OpenCourseWare),http://webcast.berkeley.edu (Accessed 10 March, 2015).

License: Creative Common BY-NC-SA 3.0.

\section{Mechanical Engineering}

Agogino, Alice M., Kellogg, Clark. Mechanical Engineering 110,2011.(University of California, Berkeley: UC Berkeley OpenCourseWare), http://webcast.berkeley.edu (Accessed 18 Jan, 2015). License: Creative Common BY-NC-SA 3.0.

Chen, Xu. Mechanical Engineering 233, 2014.(University of California, Berkeley: UC Berkeley OpenCourseWare),http://webcast.berkeley.edu (Accessed 18 Jan, 2015). License: Creative Common BY-NC-SA 3.0.

Sava, Omer. Mechanical Engineering 106, 2013 (University of California, Berkeley: UC Berkeley OpenCourseWare), http://webcast.berkeley.edu (Accessed 18 Jan, 2015). License: Creative Common BY-NC-SA 3.0.

Vijayaraghavan, Athulan. Mechanical Engineering 220, 2012 (University of California, Berkeley: UC Berkeley OpenCourseWare),http://webcast.berkeley.edu (Accessed 18 Jan, 2015). License: Creative Common BY-NC-SA 3.0.

\section{Nuclear Engineering}

Bernstein, Lee A. Nuclear Engineering 101, 2014.(University of California, Berkeley: UC Berkeley OpenCourseWare), http://webcast.berkeley.edu (Accessed 18 Jan, 2015). License: Creative Common BY-NC-SA 3.0.

\section{Public Policy}

Kammen, Daniel M. Public Policy C284, 2014. (University of California, Berkeley: UC Berkeley OpenCourseWare),http://webcast.berkeley.edu (Accessed 18 Jan, 2015). License: Creative Common BY-NC-SA 3.0.

\section{Philosophy}

Campell, John Joseph. Philosophy 135, 2014. (University of California, Berkeley: UC Berkeley OpenCourseWare),http://webcast.berkeley.edu (Accessed 18 Jan, 2015). License: Creative Common BY-NC-SA 3.0. 


\section{Appendix 14. Spoken materials from Stanford Engineering courses}

\section{Artificial Intelligence}

Khatib, Oussama. Introduction to Robotics, Winter 2007/2008. (Stanford University: Stanford Engineering Everywhere Course), https://see.stanford.edu/ (Accessed 17 Jan, 2015). License: Creative Common BY-NC-SA 4.0.

Manning, Christopher D. Natural Language Processing 2008. (Stanford University: Stanford Engineering Everywhere Course), https://see.stanford.edu/ (Accessed 17 Jan, 2015). License: Creative Common BY-NC-SA 4.0.

$\mathrm{Ng}$, Andrew. Machine Learning. (Stanford University: Stanford Engineering Everywhere Course), https://see.stanford.edu/ (Accessed 17 Jan, 2015). License: Creative Common BYNC-SA 4.0.

\section{Electrical Engineering}

Boyd, Stephen. Introduction to Linear Dynamical Systems, Fall 2007/2008. (Stanford University: Stanford Engineering Everywhere Course), https://see.stanford.edu/ (Accessed 17 Jan, 2015). License: Creative Common BY-NC-SA 4.0.

Boyd, Stephen. Convex Optimization I, Winter 2007/2008. (Stanford University: Stanford Engineering Everywhere Course), https://see.stanford.edu/ (Accessed 17 Jan, 2015). License: Creative Common BY-NC-SA 4.0.

Boyd, Stephen. Convex Optimization II, Spring 2007/2008. (Stanford University: Stanford Engineering Everywhere Course), https://see.stanford.edu/ (Accessed 17 Jan, 2015). License: Creative Common BY-NC-SA 4.0.

Osgood, Brad G. The Fourier Transform and its Applications, Fall 2007. (Stanford University: Stanford Engineering Everywhere Course), https://see.stanford.edu/ (Accessed 17 Jan, 2015). License: Creative Common BY-NC-SA 4.0.

\section{Introduction to Computer Sciences}

Cain,Jerry. Programming Paradigms CS107, Spring 2008. (Stanford University: Stanford Engineering Everywhere Course), https://see.stanford.edu/ (Accessed 17 Jan, 2015). License: Creative Common BY-NC-SA 4.0.

Sahami, Mehran. Programming Methodology, Fall 2007. (Stanford University: Stanford Engineering Everywhere Course), https://see.stanford.edu/ (Accessed 17 Jan, 2015). License: Creative Common BY-NC-SA 4.0.

Zelenski, Julie. Programming Abstractions, Spring 2008. (Stanford University: Stanford Engineering Everywhere Course), https://see.stanford.edu/ (Accessed 17 Jan, 2015). License: Creative Common BY-NC-SA 4.0. 


\section{Appendix 15. Written materials from Massachusetts Institute of Technology \\ Astronomy}

Emilio Frazzoli, and Munther Dahleh. 6.241J Dynamic Systems and Control, Spring 2011. (Massachusetts Institute of Technology: MIT

OpenCourseWare), $\underline{\text { http://ocw.mit.edu (Accessed } 20 \text { Jan, 2016). License: Creative }}$

Commons BY-NC-SA

Eric Feron, and James Paduano. 16.30 Estimation and Control of Aerospace Systems, Spring 2004. (Massachusetts Institute of Technology: MIT

OpenCourseWare), $\underline{\text { http://ocw.mit.edu (Accessed } 20 \text { Jan, 2016). License: Creative }}$ Commons BY-NC-SA

Richard Larson, Amedeo Odoni, and Arnold Barnett. 1.203J Logistical and Transportation Planning Methods, Fall 2006. (Massachusetts Institute of Technology: MIT OpenCourseWare), http://ocw.mit.edu (Accessed 20 Jan, 2016). License: Creative Commons BY-NC-SA

Robert Rines. 6.901 Inventions and Patents, Fall 2005. (Massachusetts Institute of Technology: MIT OpenCourseWare), http://ocw.mit.edu (Accessed 20 Jan, 2016). License: $\underline{\text { Creative Commons BY-NC-SA }}$

\section{Civil and Environmental Engineering}

Jerome Connor. 1.571 Structural Analysis and Control, Spring 2004. (Massachusetts Institute of Technology: MIT OpenCourseWare), http://ocw.mit.edu (Accessed 20 Jan, 2016). License: Creative Commons BY-NC-SA

Franz Hover, and Harrison Chin. 2.017J Design of Electromechanical Robotic Systems, Fall 2009. (Massachusetts Institute of Technology: MIT

OpenCourseWare), http://ocw.mit.edu (Accessed 20 Jan, 2016). License: $\underline{\text { Creative }}$ Commons BY-NC-SA

Fred Moavenzadeh. 1.040 Project Management, Spring 2009. (Massachusetts Institute of Technology: MIT OpenCourseWare), http://ocw.mit.edu (Accessed 20 Jan, 2016). License: Creative Commons BY-NC-SA

Richard Larson, Amedeo Odoni, and Arnold Barnett. 1.203J Logistical and Transportation Planning Methods, Fall 2006. (Massachusetts Institute of Technology: MIT OpenCourseWare), http://ocw.mit.edu (Accessed 20 Jan, 2016). License: Creative Commons BY-NC-SA

\section{Ecology \& Geology}

John Southard. 12.090 Introduction to Fluid Motions, Sediment Transport, and CurrentGenerated Sedimentary Structures, Fall 2006. (Massachusetts Institute of Technology: MIT OpenCourseWare), http://ocw.mit.edu (Accessed 20 Jan, 2016). License: Creative Commons BY-NC-SA

John Southard. 12.090 The Environment of the Earth's Surface, Spring 2007. (Massachusetts Institute of Technology: MIT 


\section{Appendix 15. Written materials from Massachusetts Institute of Technology (continued)}

OpenCourseWare), http://ocw.mit.edu(Accessed 20 Jan, 2016). License: $\underline{\text { Creative }}$ Commons BY-NC-SA

\section{Electrical Engineering}

Alan Oppenheim, and George Verghese. 6.011 Introduction to Communication, Control, and Signal Processing, Spring 2010. (Massachusetts Institute of Technology: MIT OpenCourseWare), http://ocw.mit.edu (Accessed 20 Jan, 2016). License: Creative Commons BY-NC-SA

David Staelin. 6.013 Electromagnetics and Applications, Spring 2009. (Massachusetts Institute of Technology: MIT OpenCourseWare), http://ocw.mit.edu (Accessed $20 \mathrm{Jan}$, 2016). License: Creative Commons BY-NC-SA

Dedric Carter, and Robert Rines. 6.931 Development of Inventions and Creative Ideas, Spring 2008. (Massachusetts Institute of Technology: MIT

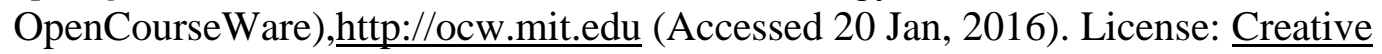
Commons BY-NC-SA

Dennis Freeman. 6.003 Signals and Systems, Fall 2011. (Massachusetts Institute of Technology: MIT OpenCourseWare), http://ocw.mit.edu (Accessed 20 Jan, 2016). License: Creative Commons BY-NC-SA

Emilio Frazzoli, and Munther Dahleh. 6.241J Dynamic Systems and Control, Spring 2011. (Massachusetts Institute of Technology: MIT OpenCourseWare), http://ocw.mit.edu (Accessed 20 Jan, 2016). License: $\underline{\text { Creative }}$ Commons BY-NC-SA

Eric Grimson, Peter Szolovits, and Trevor Darrell. 6.001 Structure and Interpretation of Computer Programs, Spring 2005. (Massachusetts Institute of Technology: MIT OpenCourseWare), http://ocw.mit.edu (Accessed 20 Jan, 2016). License: Creative Commons BY-NC-SA

Franz Kärtner. 6.977 Ultrafast Optics, Spring 2005. (Massachusetts Institute of Technology: MIT OpenCourseWare), http://ocw.mit.edu (Accessed 20 Jan, 2016). License: Creative Commons BY-NC-SA

Hari Balakrishnan, and George Verghese. 6.02 Introduction to EECS II: Digital Communication Systems, Fall 2012. (Massachusetts Institute of Technology: MIT OpenCourseWare), http://ocw.mit.edu (Accessed 20 Jan, 2016). License: Creative Commons BY-NC-SA

Harold Abelson, and Philip Greenspun. 6.171 Software Engineering for Web Applications, Fall 2003. (Massachusetts Institute of Technology: MIT

OpenCourseWare), http://ocw.mit.edu (Accessed 20 Jan, 2016). License: Creative Commons BY-NC-SA

James Kirtley Jr.. 6.061 Introduction to Electric Power Systems, Spring 2011. (Massachusetts Institute of Technology: MIT 


\section{Appendix 15. Written materials from Massachusetts Institute of Technology (continued)}

OpenCourseWare), http://ocw.mit.edu(Accessed 20 Jan, 2016). License: $\underline{\text { Creative }}$ Commons BY-NC-SA

Leslie Kaelbling, Jacob White, Harold Abelson, Dennis Freeman, Tomás Lozano-Pérez, and Isaac Chuang. 6.01SC Introduction to Electrical Engineering and Computer Science I, Spring 2011. (Massachusetts Institute of Technology: MIT OpenCourseWare), http://ocw.mit.edu (Accessed 20 Jan, 2016). License: Creative Commons BY-NC-SA

Marc Baldo. 6.701 Introduction to Nanoelectronics, Spring 2010. (Massachusetts Institute of Technology: MIT OpenCourseWare), http://ocw.mit.edu (Accessed 20 Jan, 2016). License: Creative Commons BY-NC-SA

Nanette Veilleux, Stefanie Shattuck-Hufnagel, and Alejna Brugos. 6.911 Transcribing Prosodic Structure of Spoken Utterances with ToBI, January IAP 2006. (Massachusetts Institute of Technology: MIT OpenCourseWare), http://ocw.mit.edu (Accessed 20 Jan, 2016). License: Creative Commons BY-NC-SA

Paul Penfield, and Seth Lloyd. 6.050J Information and Entropy, Spring 2008. (Massachusetts Institute of Technology: MIT OpenCourseWare), http://ocw.mit.edu(Accessed 20 Jan, 2016). License: $\underline{\text { Creative }}$ Commons BY-NC-SA

Robert Gallager. 6.262 Discrete Stochastic Processes, Spring 2011. (Massachusetts Institute of Technology: MIT OpenCourseWare), http://ocw.mit.edu (Accessed 20 Jan, 2016). License: Creative Commons BY-NC-SA

Sanjoy Mahajan. 6.055J The Art of Approximation in Science and Engineering, Spring 2008. (Massachusetts Institute of Technology: MIT OpenCourseWare),http://ocw.mit.edu (Accessed 20 Jan, 2016). License: Creative Commons BY-NC-SA

\section{Engineering Systems Division}

Fred Moavenzadeh. 1.040 Project Management, Spring 2009. (Massachusetts Institute of Technology: MIT OpenCourseWare), http://ocw.mit.edu (Accessed 20 Jan, 2016).

License: Creative Commons BY-NC-SA

\section{Maths}

Albert Meyer. 6.042J Mathematics for Computer Science, Spring 2010. (Massachusetts Institute of Technology: MIT OpenCourseWare), http://ocw.mit.edu (Accessed 20 Jan, 2016). License: Creative Commons BY-NC-SA

Andrew Sutherland. 18.782 Introduction to Arithmetic Geometry, Fall 2013. (Massachusetts Institute of Technology: MIT OpenCourseWare), http://ocw.mit.edu(Accessed 20 Jan, 2016). License: $\underline{\text { Creative }}$ Commons BY-NC-SA 


\section{Appendix 15. Written materials from Massachusetts Institute of Technology (continued)}

Daniel Kleitman. 18.013A Calculus with Applications, Spring 2005. (Massachusetts Institute of Technology: MIT OpenCourseWare), http://ocw.mit.edu (Accessed 20 Jan, 2016). License: Creative Commons BY-NC-SA

Sanjoy Mahajan. 18.098 Street-Fighting Mathematics, January IAP 2008. (Massachusetts Institute of Technology: MIT OpenCourseWare), http://ocw.mit.edu(Accessed 20 Jan, 2016). License: Creative Commons BY-NC-SA

Scott Sheffield. 18.440 Probability and Random Variables, Spring 2014. (Massachusetts Institute of Technology: MIT OpenCourseWare), http://ocw.mit.edu(Accessed 20 Jan, 2016). License: Creative Commons BY-NC-SA

\section{Material Engineering}

David Roylance. 3.11 Mechanics of Materials, Fall 1999. (Massachusetts Institute of Technology: MIT OpenCourseWare), http://ocw.mit.edu (Accessed 20 Jan, 2016).

License: Creative Commons BY-NC-SA

\section{Mechanical engineering}

Domitilla Del Vecchio. 2.18 Biomolecular Feedback Systems, Spring 2015. (Massachusetts Institute of Technology: MIT OpenCourseWare), http://ocw.mit.edu(Accessed 20 Jan, 2016). License: Creative Commons BY-NC-SA

Michael Triantafyllou. 2.154 Maneuvering and Control of Surface and Underwater Vehicles (13.49), Fall 2004. (Massachusetts Institute of Technology: MIT OpenCourseWare), http://ocw.mit.edu (Accessed 20 Jan, 2016). License: Creative Commons BY-NC-SA

Tomasz Wierzbicki. 2.080J Structural Mechanics, Fall 2013. (Massachusetts Institute of Technology: MIT OpenCourseWare), http://ocw.mit.edu (Accessed 20 Jan, 2016). License: Creative Commons BY-NC-SA

\section{Media Arts and Science}

Christopher Schmandt. MAS.632 Conversational Computer Systems, Fall 2008. (Massachusetts Institute of Technology: MIT OpenCourseWare), http://ocw.mit.edu(Accessed 20 Jan, 2016). License: $\underline{\text { Creative }}$ Commons BY-NC-SA

\section{Nuclear Science and Engineering}

Ian Hutchinson. 22.15 Essential Numerical Methods, Fall 2014. (Massachusetts Institute of Technology: MIT OpenCourseWare), http://ocw.mit.edu (Accessed 20 Jan, 2016). License: Creative Commons BY-NC-SA 


\section{Appendix 16. Members of the word family 'predict' in the three ASWL versions}

\begin{tabular}{lll}
\hline Level 2.5 word family version & Level 3 word family version & Level 6 word family version \\
\hline predict & predict & predict \\
predicted & predicted & predicted \\
predicting & predicting & predicting \\
predicts & predicts & predicts \\
& predictable & predictable \\
& predictably & predictably \\
& unpredictable & unpredictable \\
& unpredictably & unpredictably \\
& & predictability \\
& prediction \\
& predictions \\
& predictive \\
& predictor \\
& predictors \\
& unpredictability \\
& &
\end{tabular}




\section{Appendix 17. Sub-lists of the ASWL headwords at Level 1}

\begin{tabular}{|c|c|c|c|c|c|c|c|c|c|c|c|}
\hline \multicolumn{12}{|c|}{ Sub-list of function words } \\
\hline 1 & be & 26 & some & 51 & where & 76 & may & 101 & under & 126 & however \\
\hline 2 & the & 27 & one & 52 & could & 77 & second & 102 & though & 127 & round \\
\hline 3 & and & 28 & get & 53 & no & 78 & around & 103 & half & 128 & among \\
\hline 4 & $\mathrm{a}$ & 29 & about & 54 & who & 79 & certain & 104 & ten & 129 & upon \\
\hline 5 & that & 30 & with & 55 & into & 80 & each & 105 & within & 130 & nobody \\
\hline 6 & to & 31 & as & 56 & over & 81 & next & 106 & hundred & 131 & except \\
\hline 7 & you & 32 & at & 57 & than & 82 & still & 107 & twenty & 132 & below \\
\hline 8 & of & 33 & will & 58 & every & 83 & last & 108 & along & 133 & billion \\
\hline 9 & it & 34 & or & 59 & much & 84 & after & 109 & without & 134 & unless \\
\hline 10 & I & 35 & he & 60 & first & 85 & off & 110 & across & 135 & beyond \\
\hline 11 & in & 36 & all & 61 & back & 86 & both & 111 & nothing & 136 & ought \\
\hline 12 & this & 37 & like & 62 & three & 87 & plus & 112 & must & 137 & twelve \\
\hline 13 & have & 38 & because & 63 & down & 88 & nine & 113 & while & 138 & none \\
\hline 14 & we & 39 & which & 64 & through & 89 & less & 114 & during & 139 & shall \\
\hline 15 & so & 40 & from & 65 & why & 90 & zero & 115 & against & 140 & eleven \\
\hline 16 & do & 41 & by & 66 & should & 91 & six & 116 & million & 141 & underneath \\
\hline 17 & not & 42 & up & 67 & might & 92 & eight & 117 & thousand & & \\
\hline 18 & they & 43 & would & 68 & most & 93 & few & 118 & until & & \\
\hline 19 & what & 44 & out & 69 & many & 94 & away & 119 & since & & \\
\hline 20 & on & 45 & how & 70 & another & 95 & enough & 120 & although & & \\
\hline 21 & but & 46 & when & 71 & between & 96 & once & 121 & near & & \\
\hline 22 & for & 47 & more & 72 & she & 97 & such & 122 & above & & \\
\hline 23 & there & 48 & other & 73 & four & 98 & seven & 123 & thirty & & \\
\hline 24 & if & 49 & two & 74 & five & 99 & yet & 124 & behind & & \\
\hline 25 & can & 50 & any & 75 & before & 100 & either & 125 & several & & \\
\hline
\end{tabular}




\section{Appendix 17. Sub-lists of the ASWL headwords at Level 1 (continued)}

\begin{tabular}{|c|c|c|c|c|c|c|c|c|c|}
\hline \multicolumn{10}{|c|}{ Sub-list 1 of lexical words } \\
\hline 1 & go & 11 & see & 21 & yes & 31 & give & 41 & need \\
\hline 2 & here & 12 & ok & 22 & use & 32 & point & 42 & problem \\
\hline 3 & just & 13 & very & 23 & actual & 33 & let & 43 & put \\
\hline 4 & know & 14 & time & 24 & really & 34 & also & 44 & only \\
\hline 5 & say & 15 & make & 25 & take & 35 & different & 45 & case \\
\hline 6 & then & 16 & look & 26 & come & 36 & call & 46 & question \\
\hline 7 & think & 17 & want & 27 & kind & 37 & good & 47 & sort \\
\hline 8 & thing & 18 & way & 28 & mean & 38 & same & 48 & happen \\
\hline 9 & right & 19 & well & 29 & work & 39 & lot & 49 & number \\
\hline 10 & now & 20 & people & 30 & talk & 40 & little & 50 & find \\
\hline \multicolumn{10}{|c|}{ Sub-list 2 of lexical words } \\
\hline 1 & part & 11 & show & 21 & state & 31 & great & 41 & become \\
\hline 2 & try & 12 & interest & 22 & tell & 32 & long & 42 & turn \\
\hline 3 & year & 13 & bit & 23 & end & 33 & maybe & 43 & group \\
\hline 4 & start & 14 & write & 24 & probably & 34 & simple & 44 & today \\
\hline 5 & again & 15 & move & 25 & important & 35 & place & 45 & act \\
\hline 6 & change & 16 & new & 26 & world & 36 & small & 46 & able \\
\hline 7 & system & 17 & high & 27 & basic & 37 & word & 47 & read \\
\hline 8 & course & 18 & term & 28 & set & 38 & reason & 48 & class \\
\hline 9 & big & 19 & fact & 29 & particular & 39 & sense & 49 & order \\
\hline 10 & even & 20 & idea & 30 & build & 40 & line & 50 & ask \\
\hline
\end{tabular}




\section{Appendix 17. Sub-lists of the ASWL headwords at Level 1 (continued)}

\begin{tabular}{|c|c|c|c|c|c|c|c|c|c|}
\hline \multicolumn{10}{|c|}{ Sub-list 3 of lexical words } \\
\hline 1 & quite & 11 & relate & 21 & general & 31 & area & 41 & keep \\
\hline 2 & whole & 12 & water & 22 & hand & 32 & base & 42 & control \\
\hline 3 & day & 13 & guy & 23 & law & 33 & history & 43 & rate \\
\hline 4 & understand & 14 & space & 24 & exact & 34 & pretty & 44 & type \\
\hline 5 & remember & 15 & power & 25 & force & 35 & begin & 45 & country \\
\hline 6 & too & 16 & person & 26 & real & 36 & answer & 46 & left \\
\hline 7 & large & 17 & level & 27 & life & 37 & whether & 47 & grow \\
\hline 8 & add & 18 & better & 28 & run & 38 & week & 48 & nature \\
\hline 9 & form & 19 & always & 29 & late & 39 & square & 49 & seem \\
\hline 10 & possible & 20 & side & 30 & name & 40 & sure & 50 & student \\
\hline \multicolumn{10}{|c|}{ Sub-list 4 of lexical words } \\
\hline 1 & together & 11 & stuff & 21 & learn & 31 & test & 41 & already \\
\hline 2 & book & 12 & help & 22 & bring & 32 & picture & 42 & programme \\
\hline 3 & market & 13 & play & 23 & clear & 33 & never & 43 & far \\
\hline 4 & govern & 14 & cost & 24 & live & 34 & free & 44 & cause \\
\hline 5 & inform & 15 & issue & 25 & feel & 35 & study & 45 & hard \\
\hline 6 & easy & 16 & difference & 26 & win & 36 & depend & 46 & suppose \\
\hline 7 & true & 17 & body & 27 & least & 37 & school & 47 & obvious \\
\hline 8 & own & 18 & human & 28 & lead & 38 & minute & 48 & bad \\
\hline 9 & else & 19 & field & 29 & centre & 39 & computer & 49 & often \\
\hline 10 & $\operatorname{man}$ & 20 & early & 30 & follow & 40 & view & 50 & close \\
\hline
\end{tabular}




\section{Appendix 17. Sub-lists of the ASWL headwords at Level 1 (continued)}

\begin{tabular}{|c|c|c|c|c|c|c|c|c|c|}
\hline \multicolumn{10}{|c|}{ Sub-list 5 of lexical words } \\
\hline 1 & charge & 11 & rather & 21 & complete & 31 & moment & 41 & size \\
\hline 2 & pay & 12 & list & 22 & love & 32 & mention & 42 & present \\
\hline 3 & deal & 13 & arm & 23 & amount & 33 & child & 43 & usual \\
\hline 4 & care & 14 & believe & 24 & hear & 34 & break & 44 & rule \\
\hline 5 & expect & 15 & step & 25 & money & 35 & explain & 45 & sorry \\
\hline 6 & company & 16 & light & 26 & couple & 36 & piece & 46 & position \\
\hline 7 & figure & 17 & old & 27 & normal & 37 & hope & 47 & total \\
\hline 8 & science & 18 & nation & 28 & story & 38 & best & 48 & ever \\
\hline 9 & matter & 19 & allow & 29 & final & 39 & open & 49 & wave \\
\hline 10 & draw & 20 & top & 30 & imagine & 40 & low & 50 & black \\
\hline \multicolumn{10}{|c|}{ Sub-list 6 of lexical words } \\
\hline 1 & almost & 11 & notice & 21 & situation & 31 & stop & 41 & sit \\
\hline 2 & involve & 12 & white & 22 & consider & 32 & fall & 42 & choose \\
\hline 3 & hold & 13 & manage & 23 & wrong & 33 & buy & 43 & land \\
\hline 4 & death & 14 & head & 24 & nice & 34 & thank & 44 & speak \\
\hline 5 & lose & 15 & inside & 25 & continue & 35 & house & 45 & meet \\
\hline 6 & plan & 16 & quick & 26 & woman & 36 & room & 46 & stand \\
\hline 7 & single & 17 & public & 27 & mind & 37 & city & 47 & health \\
\hline 8 & pick & 18 & home & 28 & short & 38 & decide & 48 & main \\
\hline 9 & guess & 19 & air & 29 & star & 39 & leave & 49 & local \\
\hline 10 & pass & 20 & degree & 30 & experience & 40 & key & 50 & hour \\
\hline
\end{tabular}




\section{Appendix 17. Sub-lists of the ASWL headwords at Level 1 (continued)}

\begin{tabular}{|c|c|c|c|c|c|c|c|c|c|}
\hline \multicolumn{10}{|c|}{ Sub-list 7 of lexical words } \\
\hline 1 & sell & 11 & perhaps & 21 & educate & 31 & sign & 41 & agree \\
\hline 2 & difficult & 12 & strong & 22 & tend & 32 & note & 42 & kill \\
\hline 3 & paper & 13 & tree & 23 & game & 33 & face & 43 & weight \\
\hline 4 & spend & 14 & send & 24 & share & 34 & middle & 44 & age \\
\hline 5 & drive & 15 & cut & 25 & ground & 35 & carry & 45 & instead \\
\hline 6 & fast & 16 & express & 26 & necessary & 36 & family & 46 & special \\
\hline 7 & front & 17 & absolute & 27 & red & 37 & huge & 47 & load \\
\hline 8 & die & 18 & return & 28 & full & 38 & rights & 48 & worry \\
\hline 9 & fair & 19 & business & 29 & wall & 39 & toward & 49 & protect \\
\hline 10 & ago & 20 & deep & 30 & job & 40 & suggest & 50 & laugh \\
\hline \multicolumn{10}{|c|}{ Sub-list 8 of lexical words } \\
\hline 1 & south & 11 & walk & 21 & support & 31 & choice & 41 & party \\
\hline 2 & perfect & 12 & feed & 22 & drop & 32 & heat & 42 & bar \\
\hline 3 & cross & 13 & rest & 23 & table & 33 & record & 43 & hit \\
\hline 4 & bottom & 14 & check & 24 & stage & 34 & fine & 44 & collect \\
\hline 5 & cover & 15 & service & 25 & young & 35 & month & 45 & night \\
\hline 6 & concern & 16 & member & 26 & treat & 36 & appear & 46 & fight \\
\hline 7 & store & 17 & page & 27 & web & 37 & count & 47 & plant \\
\hline 8 & alright & 18 & car & 28 & past & 38 & wait & 48 & double \\
\hline 9 & major & 19 & board & 29 & stay & 39 & raise & 49 & happy \\
\hline 10 & shape & 20 & north & 30 & animal & 40 & especially & 50 & drug \\
\hline
\end{tabular}




\section{Appendix 17. Sub-lists of the ASWL headwords at Level 1 (continued)}

\begin{tabular}{|c|c|c|c|c|c|c|c|c|c|}
\hline \multicolumn{10}{|c|}{ Sub-list 9 of lexical words } \\
\hline 1 & fun & 11 & sound & 21 & mark & 31 & pair & 41 & fly \\
\hline 2 & push & 12 & fix & 22 & wide & 32 & indeed & 42 & stick \\
\hline 3 & wonder & 13 & friend & 23 & fit & 33 & street & 43 & blue \\
\hline 4 & sex & 14 & rise & 24 & forget & 34 & accept & 44 & fill \\
\hline 5 & pull & 15 & watch & 25 & kid & 35 & doctor & 45 & worth \\
\hline 6 & team & 16 & serve & 26 & subject & 36 & chance & 46 & discover \\
\hline 7 & further & 17 & worse & 27 & finish & 37 & definite & 47 & poor \\
\hline 8 & parent & 18 & train & 28 & report & 38 & straight & 48 & reach \\
\hline 9 & slow & 19 & realise & 29 & gas & 39 & post & 49 & miss \\
\hline 10 & cool & 20 & colour & 30 & safe & 40 & edge & 50 & slight \\
\hline \multicolumn{10}{|c|}{ Sub-list 10 of lexical words } \\
\hline 1 & forward & 11 & hello & 21 & movie & 31 & door & 41 & baby \\
\hline 2 & rough & 12 & ahead & 22 & serious & 32 & fish & 42 & drink \\
\hline 3 & green & 13 & office & 23 & surprise & 33 & pack & 43 & dead \\
\hline 4 & farm & 14 & throw & 24 & owned & 34 & fire & 44 & extra \\
\hline 5 & ready & 15 & god & 25 & date & 35 & judge & 45 & hole \\
\hline 6 & offer & 16 & please & 26 & letter & 36 & danger & 46 & west \\
\hline 7 & eye & 17 & box & 27 & enter & 37 & $\operatorname{tax}$ & 47 & strike \\
\hline 8 & responsible & 18 & photograph & 28 & save & 38 & beauty & 48 & proper \\
\hline 9 & cold & 19 & recent & 29 & morning & 39 & pain & 49 & apart \\
\hline 10 & self & 20 & foot & 30 & track & 40 & employ & 50 & bear \\
\hline
\end{tabular}




\section{Appendix 17. Sub-lists of the ASWL headwords at Level 1 (continued)}

\begin{tabular}{|c|c|c|c|c|c|c|c|c|c|}
\hline \multicolumn{10}{|c|}{ Sub-list 11 of lexical words } \\
\hline 1 & island & 11 & lay & 21 & burn & 31 & aware & 41 & road \\
\hline 2 & hot & 12 & advertise & 22 & amaze & 32 & mother & 42 & birth \\
\hline 3 & oil & 13 & catch & 23 & trouble & 33 & ship & 43 & news \\
\hline 4 & ball & 14 & arrange & 24 & clock & 34 & police & 44 & summer \\
\hline 5 & east & 15 & sudden & 25 & secure & 35 & rain & 45 & spring \\
\hline 6 & prepare & 16 & bill & 26 & lie & 36 & dark & 46 & king \\
\hline 7 & soon & 17 & shoot & 27 & travel & 37 & town & 47 & jump \\
\hline 8 & born & 18 & river & 28 & hang & 38 & fortunate & 48 & listen \\
\hline 9 & telephone & 19 & excite & 29 & fear & 39 & neighbour & 49 & music \\
\hline 10 & heavy & 20 & race & 30 & video & 40 & closed & 50 & park \\
\hline \multicolumn{10}{|c|}{ Sub-list 12 of lexical words } \\
\hline 1 & blow & 11 & mistake & 21 & television & 31 & handle & 41 & noise \\
\hline 2 & pound & 12 & station & 22 & conversation & 32 & Wednesday & 42 & father \\
\hline 3 & Thursday & 13 & rich & 23 & waste & 33 & wear & 43 & tape \\
\hline 4 & shop & 14 & rid & 24 & mile & 34 & press & 44 & Friday \\
\hline 5 & expensive & 15 & ring & 25 & master & 35 & beat & 45 & corner \\
\hline 6 & window & 16 & suit & 26 & luck & 36 & alone & 46 & smoke \\
\hline 7 & visit & 17 & Monday & 27 & tie & 37 & egg & 47 & rock \\
\hline 8 & touch & 18 & truth & 28 & spot & 38 & bright & 48 & Tuesday \\
\hline 9 & join & 19 & college & 29 & flat & 39 & boy & 49 & brown \\
\hline 10 & terrible & 20 & trust & 30 & quarter & 40 & floor & 50 & bus \\
\hline
\end{tabular}




\section{Appendix 17. Sub-lists of the ASWL headwords at Level 1 (continued)}

\begin{tabular}{|c|c|c|c|c|c|c|c|c|c|}
\hline \multicolumn{10}{|c|}{ Sub-list 13 of lexical words } \\
\hline 1 & odd & 11 & roll & 21 & dream & 31 & girl & 41 & hospital \\
\hline 2 & promise & 12 & skin & 22 & crazy & 32 & marry & 42 & thick \\
\hline 3 & son & 13 & strange & 23 & doubt & 33 & mister & 43 & $\operatorname{sir}$ \\
\hline 4 & gold & 14 & mess & 24 & $\operatorname{dog}$ & 34 & tail & 44 & hide \\
\hline 5 & enjoy & 15 & glass & 25 & gun & 35 & card & 45 & wood \\
\hline 6 & empty & 16 & wish & 26 & forest & 36 & settle & 46 & ride \\
\hline 7 & apparent & 17 & hat & 27 & brother & 37 & sleep & 47 & hair \\
\hline 8 & pop & 18 & lift & 28 & comfort & 38 & dry & 48 & yellow \\
\hline 9 & lake & 19 & arrive & 29 & tight & 39 & wife & 49 & hunt \\
\hline 10 & lock & 20 & cheap & 30 & wine & 40 & bed & 50 & tomorrow \\
\hline \multicolumn{10}{|c|}{ Sub-list 14 of lexical words } \\
\hline 1 & voice & 11 & wheel & 21 & officer & 31 & afraid & & \\
\hline 2 & mountain & 12 & hate & 22 & hall & 32 & fellow & & \\
\hline 3 & radio & 13 & weather & 23 & grandfather & 33 & afternoon & & \\
\hline 4 & internet & 14 & boat & 24 & wake & 34 & bag & & \\
\hline 5 & shake & 15 & shut & 25 & milk & 35 & hurt & & \\
\hline 6 & honour & 16 & seat & 26 & excuse & 36 & freeze & & \\
\hline 7 & leg & 17 & favourite & 27 & mouth & 37 & honest & & \\
\hline 8 & song & 18 & hell & 28 & sport & 38 & trip & & \\
\hline 9 & yesterday & 19 & taste & 29 & admit & 39 & awful & & \\
\hline 10 & horse & 20 & sick & 30 & sky & & & & \\
\hline
\end{tabular}


Appendix 18. Sub-lists of the ASWL headwords at Level 2

\begin{tabular}{|c|c|c|c|c|c|c|c|c|c|}
\hline \multicolumn{10}{|c|}{ Sub-list of function words } \\
\hline 1 & minus & 2 & per & 3 & whereas & 4 & forth & 5 & aside \\
\hline \multicolumn{10}{|c|}{ Sub-list 1 of lexical words } \\
\hline 1 & example & 11 & create & 21 & current & 31 & language & 41 & common \\
\hline 2 & equal & 12 & increase & 22 & economy & 32 & property & 42 & discuss \\
\hline 3 & value & 13 & measure & 23 & material & 33 & represent & 43 & century \\
\hline 4 & energy & 14 & politics & 24 & apply & 34 & condition & 44 & refer \\
\hline 5 & product & 15 & produce & 25 & environment & 35 & industry & 45 & project \\
\hline 6 & process & 16 & argue & 26 & result & 36 & social & 46 & pressure \\
\hline 7 & develop & 17 & vary & 27 & effect & 37 & period & 47 & culture \\
\hline 8 & operate & 18 & individual & 28 & percent & 38 & unit & 48 & unite \\
\hline 9 & model & 19 & design & 29 & surface & 39 & exist & 49 & society \\
\hline 10 & states & 20 & direction & 30 & require & 40 & constant & 50 & react \\
\hline \multicolumn{10}{|c|}{ Sub-list 2 of lexical words } \\
\hline 1 & lower & 11 & research & 21 & assume & 31 & correct & 41 & section \\
\hline 2 & divide & 12 & active & 22 & limit & 32 & detail & 42 & associate \\
\hline 3 & specific & 13 & calculate & 23 & combine & 33 & length & 43 & introduce \\
\hline 4 & object & 14 & flow & 24 & image & 34 & capital & 44 & engineer \\
\hline 5 & compare & 15 & standard & 25 & decision & 35 & affect & 45 & reduce \\
\hline 6 & mass & 16 & therefore & 26 & likely & 36 & risk & 46 & determine \\
\hline 7 & positive & 17 & occur & 27 & describe & 37 & electric & 47 & resist \\
\hline 8 & similar & 18 & provide & 28 & technology & 38 & community & 48 & labour \\
\hline 9 & connect & 19 & identify & 29 & distance & 39 & pattern & 49 & observe \\
\hline 10 & organize & 20 & include & 30 & scale & 40 & direct & 50 & region \\
\hline
\end{tabular}




\section{Appendix 18. Sub-lists of the ASWL headwords at Level 2 (continued)}

\begin{tabular}{|c|c|c|c|c|c|c|c|c|c|}
\hline \multicolumn{10}{|c|}{ Sub-list 3 of lexical words } \\
\hline 1 & average & 11 & feature & 21 & future & 31 & copy & 41 & maximum \\
\hline 2 & memory & 12 & complicate & 22 & path & 32 & extreme & 42 & bunch \\
\hline 3 & entire & 13 & map & 23 & character & 33 & event & 43 & block \\
\hline 4 & separate & 14 & modern & 24 & bind & 34 & brain & 44 & respect \\
\hline 5 & slide & 15 & role & 25 & supply & 35 & male & 45 & search \\
\hline 6 & typical & 16 & select & 26 & mathematics & 36 & access & 46 & university \\
\hline 7 & perform & 17 & various & 27 & success & 37 & concentrate & 47 & dollar \\
\hline 8 & approach & 18 & stable & 28 & claim & 38 & range & 48 & recognize \\
\hline 9 & physical & 19 & locate & 29 & practise & 39 & speed & 49 & benefit \\
\hline 10 & signal & 20 & policy & 30 & original & 40 & site & 50 & basis \\
\hline \multicolumn{10}{|c|}{ Sub-list 4 of lexical words } \\
\hline 1 & purpose & 11 & account & 21 & remain & 31 & president & 41 & fail \\
\hline & demand & 12 & remove & 22 & evidence & 32 & challenge & 42 & match \\
\hline 3 & root & 13 & famous & 23 & previous & 33 & classic & 43 & contract \\
\hline 4 & survive & 14 & vote & 24 & due & 34 & knowledge & 44 & option \\
\hline 5 & quality & 15 & metre & 25 & version & 35 & stress & 45 & maintain \\
\hline 6 & female & 16 & balance & 26 & prove & 36 & switch & 46 & establish \\
\hline 7 & goal & 17 & eventually & 27 & topic & 37 & justice & 47 & commit \\
\hline 8 & prime & 18 & circle & 28 & advantage & 38 & indicate & 48 & replace \\
\hline 9 & available & 19 & laboratory & 29 & opportunity & 39 & contain & 49 & attention \\
\hline 10 & tradition & 20 & quote & 30 & improve & 40 & series & 50 & private \\
\hline
\end{tabular}




\section{Appendix 18. Sub-lists of the ASWL headwords at Level 2 (continued)}

\begin{tabular}{|c|c|c|c|c|c|c|c|c|c|}
\hline \multicolumn{10}{|c|}{ Sub-list 5 of lexical words } \\
\hline 1 & cousin & 11 & immediate & 21 & oppose & 31 & remind & 41 & chapter \\
\hline 2 & comment & 12 & regard & 22 & style & 32 & military & 42 & instance \\
\hline 3 & generation & 13 & print & 23 & effort & 33 & medical & 43 & progress \\
\hline 4 & competition & 14 & gain & 24 & spread & 34 & prefer & 44 & grade \\
\hline 5 & influence & 15 & rely & 25 & department & 35 & exchange & 45 & pure \\
\hline 6 & wire & 16 & attract & 26 & loss & 36 & article & 46 & split \\
\hline 7 & shift & 17 & repeat & 27 & according & 37 & otherwise & 47 & background \\
\hline 8 & exam & 18 & profession & 28 & desire & 38 & citizen & 48 & regular \\
\hline 9 & release & 19 & attack & 29 & file & 39 & customer & 49 & income \\
\hline 10 & expose & 20 & $\operatorname{mix}$ & 30 & damage & 40 & fund & 50 & familiar \\
\hline \multicolumn{10}{|c|}{ Sub-list 6 of lexical words } \\
\hline 1 & contribute & 11 & screen & 21 & upper & 31 & channel & 41 & threat \\
\hline 2 & row & 12 & message & 22 & incredible & 32 & engage & 42 & avoid \\
\hline 3 & detect & 13 & broad & 23 & instruct & 33 & attach & 43 & edit \\
\hline 4 & elect & 14 & exercise & 24 & skill & 34 & cent & 44 & receive \\
\hline 5 & confuse & 15 & transfer & 25 & stream & 35 & directed & 45 & propose \\
\hline 6 & seconds & 16 & popular & 26 & sheet & 36 & drama & 46 & item \\
\hline 7 & opposite & 17 & brief & 27 & weak & 37 & interview & 47 & height \\
\hline 8 & intense & 18 & chain & 28 & trick & 38 & rapid & 48 & recommend \\
\hline 9 & prevent & 19 & harm & 29 & plate & 39 & satisfy & 49 & destroy \\
\hline 10 & contact & 20 & ray & 30 & attempt & 40 & capable & 50 & solid \\
\hline
\end{tabular}




\section{Appendix 18. Sub-lists of the ASWL headwords at Level 2 (continued)}

\begin{tabular}{|c|c|c|c|c|c|c|c|c|c|}
\hline \multicolumn{10}{|c|}{ Sub-list 7 of lexical words } \\
\hline 1 & scene & 11 & adult & 21 & extend & 31 & mate & 41 & thin \\
\hline 2 & conscious & 12 & illustrate & 22 & mill & 32 & gate & 42 & coast \\
\hline 3 & advance & 13 & email & 23 & branch & 33 & suffer & 43 & grant \\
\hline 4 & practical & 14 & opinion & 24 & enormous & 34 & appeal & 44 & seek \\
\hline 5 & title & 15 & library & 25 & battle & 35 & tiny & 45 & register \\
\hline 6 & commerce & 16 & mental & 26 & deliver & 36 & attitude & 46 & adapt \\
\hline 7 & score & 17 & foreign & 27 & western & 37 & guide & 47 & encourage \\
\hline 8 & speech & 18 & defence & 28 & recall & 38 & weird & 48 & emotion \\
\hline 9 & remark & 19 & agent & 29 & band & 39 & massive & 49 & surround \\
\hline 10 & folk & 20 & shock & 30 & circumstance & 40 & somewhat & 50 & guarantee \\
\hline \multicolumn{10}{|c|}{ Sub-list 8 of lexical words } \\
\hline 1 & rare & 11 & ignore & 21 & ill & 31 & reserve & 41 & plastic \\
\hline 2 & strength & 12 & lack & 22 & automatic & 32 & career & 42 & schedule \\
\hline 3 & command & 13 & favour & 23 & convince & 33 & ordinary & 43 & intend \\
\hline 4 & bay & 14 & minor & 24 & instrument & 34 & injure & 44 & occasion \\
\hline 5 & trial & 15 & bomb & 25 & wing & 35 & sale & 45 & examine \\
\hline 6 & official & 16 & peace & 26 & credit & 36 & alive & 46 & port \\
\hline 7 & presume & 17 & super & 27 & recover & 37 & smooth & 47 & excellent \\
\hline 8 & smart & 18 & sugar & 28 & committee & 38 & float & 48 & announce \\
\hline 9 & equipment & 19 & pause & 29 & intent & 39 & wrap & 49 & pump \\
\hline 10 & fashion & 20 & accident & 30 & depress & 40 & discipline & 50 & salt \\
\hline
\end{tabular}




\section{Appendix 18. Sub-lists of the ASWL headwords at Level 2 (continued)}

\begin{tabular}{|c|c|c|c|c|c|c|c|c|c|}
\hline \multicolumn{10}{|c|}{ Sub-list 9 of lexical words } \\
\hline 1 & bridge & 11 & investigate & 21 & disturb & 31 & pool & 41 & truck \\
\hline 2 & escape & 12 & steady & 22 & belong & 32 & instant & 42 & staff \\
\hline 3 & bend & 13 & welcome & 23 & attend & 33 & mail & 43 & warn \\
\hline 4 & struggle & 14 & medicine & 24 & newspaper & 34 & alcohol & 44 & hire \\
\hline 5 & flip & 15 & aid & 25 & apple & 35 & tour & 45 & spirit \\
\hline 6 & camera & 16 & tense & 26 & wise & 36 & pole & 46 & deny \\
\hline 7 & disappear & 17 & lesson & 27 & secret & 37 & lawyer & 47 & screw \\
\hline 8 & stretch & 18 & tough & 28 & counter & 38 & relax & 48 & possess \\
\hline 9 & plug & 19 & trace & 29 & native & 39 & curious & 49 & council \\
\hline 10 & clever & 20 & purchase & 30 & pleasure & 40 & narrow & 50 & manner \\
\hline
\end{tabular}




\section{Appendix 19. Sub-lists of the ASWL headwords at Level 3}

\section{No function words}

\begin{tabular}{|c|c|c|c|c|c|c|c|c|c|}
\hline \multicolumn{10}{|c|}{ Sub-list 1 of lexical words } \\
\hline 1 & function & 11 & analyse & 21 & experiment & 31 & interact & 41 & context \\
\hline 2 & structure & 12 & relative & 22 & distribute & 32 & dimension & 42 & constrain \\
\hline 3 & define & 13 & negative & 23 & regulate & 33 & strategy & 43 & generate \\
\hline 4 & data & 14 & solve & 24 & dense & 34 & angle & 44 & effective \\
\hline 5 & lecture & 15 & potential & 25 & concept & 35 & frequency & 45 & impact \\
\hline 6 & equation & 16 & solution & 26 & complex & 36 & derive & 46 & initial \\
\hline 7 & theory & 17 & source & 27 & curve & 37 & principle & 47 & communicate \\
\hline 8 & method & 18 & essential & 28 & behaviour & 38 & link & 48 & estimate \\
\hline 9 & element & 19 & response & 29 & focus & 39 & resource & 49 & phase \\
\hline 10 & factor & 20 & temperature & 30 & construct & 40 & sequence & 50 & invest \\
\hline
\end{tabular}




\section{Appendix 19. Sub-lists of the ASWL headwords at Level 3 (continued)}

\begin{tabular}{|c|c|c|c|c|c|c|c|c|c|}
\hline \multicolumn{10}{|c|}{ Sub-list 2 of lexical words } \\
\hline 1 & assign & 11 & consume & 21 & precise & 31 & plot & 41 & etc \\
\hline 2 & predict & 12 & formula & 22 & compete & 32 & review & 42 & distinct \\
\hline 3 & text & 13 & transform & 23 & assumption & 33 & genetic & 43 & integrate \\
\hline 4 & global & 14 & primary & 24 & error & 34 & fundamental & 44 & rational \\
\hline 5 & component & 15 & evolution & 25 & independent & 35 & coordinate & 45 & notion \\
\hline 6 & reflect & 16 & efficient & 26 & mechanic & 36 & motion & 46 & radiate \\
\hline 7 & random & 17 & volume & 27 & civil & 37 & evaluate & 47 & extent \\
\hline 8 & cycle & 18 & significant & 28 & critic & 38 & ratio & 48 & conduct \\
\hline 9 & versus & 19 & chemical & 29 & quantity & 39 & interpret & 49 & implement \\
\hline 10 & sum & 20 & philosophy & 30 & international & 40 & authority & 50 & target \\
\hline
\end{tabular}

\begin{tabular}{|c|c|c|c|c|c|c|c|c|c|}
\hline \multicolumn{10}{|c|}{ Sub-list 3 of lexical words } \\
\hline 1 & professor & 11 & respond & 21 & failure & 31 & publish & 41 & minimum \\
\hline 2 & category & 12 & task & 22 & description & 32 & logic & 42 & demonstrate \\
\hline 3 & achieve & 13 & emit & 23 & symbol & 33 & scheme & 43 & conflict \\
\hline 4 & congress & 14 & accurate & 24 & capacity & 34 & majority & 44 & technique \\
\hline 5 & mechanism & 15 & nuclear & 25 & decrease & 35 & approximate & 45 & debate \\
\hline 6 & multiple & 16 & characteristic & 26 & correspond & 36 & sample & 46 & oriented \\
\hline 7 & label & 17 & device & 27 & colony & 37 & graduate & 47 & perspective \\
\hline 8 & expand & 18 & assess & 28 & ultimate & 38 & internal & 48 & document \\
\hline 9 & statistic & 19 & institution & 29 & technical & 39 & consequence & 49 & corporate \\
\hline 10 & aspect & 20 & proportion & 30 & invent & 40 & net & 50 & constitution \\
\hline
\end{tabular}




\section{Appendix 19. Sub-lists of the ASWL headwords at Level 3 (continued)}

\begin{tabular}{|c|c|c|c|c|c|c|c|c|c|}
\hline \multicolumn{10}{|c|}{ Sub-list 4 of lexical words } \\
\hline 1 & origin & 11 & behave & 21 & restrict & 31 & outcome & 41 & crisis \\
\hline 2 & variety & 12 & promote & 22 & index & 32 & evolve & 42 & rank \\
\hline 3 & appropriate & 13 & objective & 23 & convert & 33 & cancel & 43 & visual \\
\hline 4 & content & 14 & alternative & 24 & sustain & 34 & transmit & 44 & unique \\
\hline 5 & transition & 15 & network & 25 & motive & 35 & reverse & 45 & slope \\
\hline 6 & hypothesis & 16 & overall & 26 & radical & 36 & abstract & 46 & audience \\
\hline 7 & relevant & 17 & gravity & 27 & boundary & 37 & core & 47 & explore \\
\hline 8 & layer & 18 & external & 28 & entry & 38 & convention & 48 & substitute \\
\hline 9 & ideal & 19 & emerge & 29 & violate & 39 & absorb & 49 & wealth \\
\hline 10 & capture & 20 & equivalent & 30 & procedure & 40 & accelerate & 50 & literal \\
\hline \multicolumn{10}{|c|}{ Sub-list 5 of lexical words } \\
\hline 1 & budget & 11 & formal & 21 & distinguish & 31 & sensitive & 41 & expansion \\
\hline 2 & modify & 12 & adopt & 22 & conserve & 32 & correlate & 42 & vision \\
\hline 3 & segment & 13 & explicit & 23 & gap & 33 & transport & 43 & sector \\
\hline 4 & belief & 14 & barrier & 24 & reject & 34 & participate & 44 & aim \\
\hline 5 & summary & 15 & impose & 25 & agriculture & 35 & bias & 45 & session \\
\hline 6 & biological & 16 & eliminate & 26 & consistent & 36 & crop & 46 & visible \\
\hline 7 & religion & 17 & chart & 27 & survey & 37 & mode & 47 & ancient \\
\hline 8 & theme & 18 & consumption & 28 & decline & 38 & phenomenon & 48 & division \\
\hline 9 & goods & 19 & neutral & 29 & justify & 39 & false & 49 & intellectual \\
\hline 10 & conclusion & 20 & isolate & 30 & liquid & 40 & update & 50 & decade \\
\hline
\end{tabular}




\section{Appendix 19. Sub-lists of the ASWL headwords at Level 3 (continued)}

\begin{tabular}{|c|c|c|c|c|c|c|c|c|c|}
\hline \multicolumn{10}{|c|}{ Sub-list 6 of lexical words } \\
\hline 1 & expert & 11 & mortal & 21 & manipulate & 31 & peak & 41 & negotiate \\
\hline 2 & emphasise & 12 & dominant & 22 & monitor & 32 & exception & 42 & valid \\
\hline 3 & sufficient & 13 & software & 23 & cooperate & 33 & reside & 43 & occupy \\
\hline 4 & proof & 14 & religious & 24 & incorporate & 34 & host & 44 & prize \\
\hline 5 & legitimate & 15 & reform & 25 & academy & 35 & author & 45 & uniform \\
\hline 6 & literature & 16 & status & 26 & factory & 36 & circulate & 46 & module \\
\hline 7 & severe & 17 & implicate & 27 & former & 37 & defend & 47 & dominate \\
\hline 8 & diverse & 18 & compensate & 28 & specify & 38 & importance & 48 & conclude \\
\hline 9 & founded & 19 & contrast & 29 & extract & 39 & rail & 49 & imply \\
\hline 10 & commission & 20 & zone & 30 & compose & 40 & electronic & 50 & media \\
\hline \multicolumn{10}{|c|}{ Sub-list 7 of lexical words } \\
\hline 1 & facility & 11 & presence & 21 & legislate & 31 & migrate & 41 & strict \\
\hline 2 & essay & 12 & exploit & 22 & controversy & 32 & acquire & 42 & disc \\
\hline 3 & adjust & 13 & theoretical & 23 & constitute & 33 & foundation & 43 & mobile \\
\hline 4 & landscape & 14 & fertile & 24 & scatter & 34 & interfere & 44 & liberal \\
\hline 5 & extraordinary & 15 & mere & 25 & miner & 35 & underlie & 45 & assembly \\
\hline 6 & tube & 16 & intervene & 26 & framework & 36 & colleague & 46 & profile \\
\hline 7 & administration & 17 & violence & 27 & criteria & 37 & insight & 47 & frequent \\
\hline 8 & restore & 18 & portion & 28 & urban & 38 & conservative & 48 & campaign \\
\hline 9 & collapse & 19 & incentive & 29 & vast & 39 & tremendous & 49 & substance \\
\hline 10 & digital & 20 & reward & 30 & display & 40 & arise & 50 & mature \\
\hline
\end{tabular}




\section{Appendix 19. Sub-lists of the ASWL headwords at Level 3 (continued)}

\begin{tabular}{|c|c|c|c|c|c|c|c|c|c|}
\hline \multicolumn{10}{|c|}{ Sub-list 8 of lexical words } \\
\hline 1 & panel & 7 & territory & 13 & pose & 19 & contradict & 25 & journal \\
\hline 2 & era & 8 & initiate & 14 & sophisticated & 20 & trend & 26 & formation \\
\hline 3 & obtain & 9 & virtual & 15 & perception & 21 & differ & 27 & fragment \\
\hline 4 & crucial & 10 & route & 16 & principal & 22 & client & 28 & amend \\
\hline 5 & prior & 11 & ton & 17 & beam & 23 & inject & 29 & cluster \\
\hline 6 & passage & 12 & yield & 18 & excess & 24 & approve & 30 & Eastern \\
\hline
\end{tabular}




\section{Appendix 20. Sub-lists of the ASWL headwords at Level 4}

No sub-list of function words

\begin{tabular}{|c|c|c|c|c|c|c|c|c|}
\hline \multicolumn{9}{|c|}{ Sub-list 1 of lexical words } \\
\hline Ranking & Word families & BNC/COCA levels & Ranking & Word families & BNC/COCA levels & Ranking & Word families & BNC/COCA levels \\
\hline 1 & rotate & 4 & 18 & exponential & 9 & 35 & norm & 4 \\
\hline 2 & linear & 4 & 19 & biology & 4 & 36 & arrow & 4 \\
\hline 3 & delta & 5 & 20 & coefficient & 5 & 37 & identical & 4 \\
\hline 4 & multiply & 4 & 21 & et al. & 4 & 38 & campus & 4 \\
\hline 5 & node & 5 & 22 & so-called & 26 & 39 & arbitrary & 5 \\
\hline 8 & alpha & 4 & 25 & notate & 6 & 42 & inverse & 7 \\
\hline 9 & velocity & 5 & 26 & differential & 5 & 43 & gamma & 7 \\
\hline 10 & diagram & 4 & 27 & optimise & 6 & 44 & fraction & 4 \\
\hline 11 & graph & 4 & 28 & geometry & 4 & 45 & intuitive & 6 \\
\hline 12 & parameter & 4 & 29 & beta & 5 & 46 & vertical & 4 \\
\hline 16 & radius & 6 & 33 & triangle & 4 & 50 & align & 4 \\
\hline 17 & semester & 10 & 34 & polar & 4 & & & \\
\hline
\end{tabular}




\section{Appendix 20. Sub-lists of the ASWL headwords at Level 4 (continued)}

\begin{tabular}{|c|c|c|c|c|c|c|c|c|}
\hline \multicolumn{9}{|c|}{ Sub-list 2 of lexical words } \\
\hline Ranking & Word families & BNC/COCA levels & Ranking & Word families & $\mathrm{BNC} / \mathrm{COCA}$ levels & Ranking & Word families & BNC/COCA levels \\
\hline 1 & simulate & 4 & 10 & interface & 4 & 19 & infer & 4 \\
\hline 2 & dynamics & 5 & 11 & straightforward & 4 & 20 & saturate & 5 \\
\hline 3 & chunk & 4 & 12 & interval & 4 & 21 & micro & 6 \\
\hline 4 & automobile & 4 & 13 & dependence & 4 & 22 & subtract & 7 \\
\hline 5 & converge & 5 & 14 & induce & 4 & 23 & scenario & 4 \\
\hline 6 & maximise & 4 & 15 & distort & 4 & 24 & analytic & 5 \\
\hline 7 & elastic & 5 & 16 & synthesis & 4 & 25 & tutor & 4 \\
\hline 8 & displace & 4 & 17 & residue & 4 & & & \\
\hline 9 & horizontal & 4 & 18 & optic & 4 & & & \\
\hline
\end{tabular}




\section{Appendix 21. Publicly accessible corpora}

\begin{tabular}{lll}
\hline Source & Kind of texts & Source \\
\hline BASE & Academic spoken, British English & https://the.sketchengine.co.uk/bonito/run.cgi/first_form?corpname=preloaded/base \\
MICASE & Academic spoken, American English & http://quod.lib.umich.edu/cgi/c/corpus/corpus?c=micase;page=simple \\
BAWE & Academic written, British English & https://the.sketchengine.co.uk/bonito/corpus/first_form?corpname=preloaded/bawe2 \\
MICUSP & Academic written, American English & http://www.helsinki.fi/varieng/CoRD/corpora/MICUSP/ \\
VOICE & General, academic spoken, English as a lingua franca & http://www.univie.ac.at/voice/page/what_is_voice \\
\hline
\end{tabular}

\section{Appendix 22. Sources of open courseware and TED Talks}

\begin{tabular}{ll}
\hline Source of material & Website \\
\hline University of California, Berkeley Open courseware & http://webcast.berkeley.edu/ \\
Massachusetts Institute of Technology open courseware & http://ocw.mit.edu/index.htm \\
Stanford University open course ware & https://see.stanford.edu/ \\
Yale University open courseware & http://oyc.yale.edu/ \\
TED Talks & https://www.ted.com/talks \\
\hline
\end{tabular}

